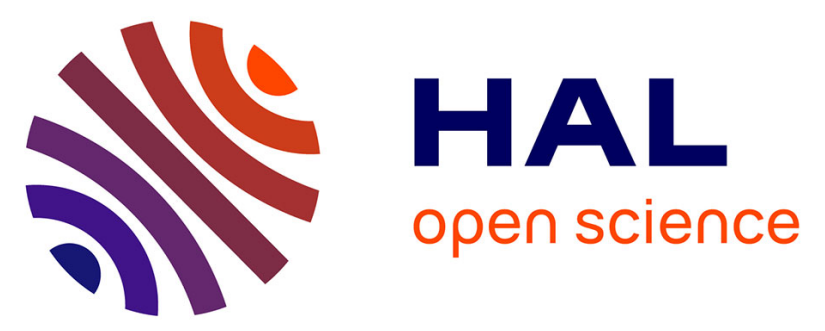

\title{
Self-Assembled Cages from the Electroactive Bis(pyrrolo)tetrathiafulvalene (BPTTF) Building Block
}

Sébastien Bivaud, Sébastien Goeb, Jean-Yves Balandier, Marcos Chas, Magali Allain, Marc Sallé

\section{- To cite this version:}

Sébastien Bivaud, Sébastien Goeb, Jean-Yves Balandier, Marcos Chas, Magali Allain, et al.. SelfAssembled Cages from the Electroactive Bis(pyrrolo)tetrathiafulvalene (BPTTF) Building Block. European Journal of Inorganic Chemistry, 2014, 2014 (14), pp.2440-2448. 10.1002/ejic.201400060 . hal-03347294

\section{HAL Id: hal-03347294 \\ https://univ-angers.hal.science/hal-03347294}

Submitted on 17 Sep 2021

HAL is a multi-disciplinary open access archive for the deposit and dissemination of scientific research documents, whether they are published or not. The documents may come from teaching and research institutions in France or abroad, or from public or private research centers.
L'archive ouverte pluridisciplinaire HAL, est destinée au dépôt et à la diffusion de documents scientifiques de niveau recherche, publiés ou non, émanant des établissements d'enseignement et de recherche français ou étrangers, des laboratoires publics ou privés. 


\title{
Self-Assembled Cages from the electroactive Bis(pyrrolo)tetrathiafulvalene (BPTTF) building block
}

\author{
Sébastien Bivaud, Sébastien Goeb, Jean-Yves Balandier, Marcos Chas, Magali Allain and Marc \\ Sallé*
}

Keywords: Cages / Self-Assembly / Coordination / Tetrathiafulvalene / Redox-active

A family of di- and tetratopic redox-active ligands designed to generate discrete self-assembled metalla-cages is described, including UV-vis and cyclic voltammetry studies, X-ray diffraction on single crystals and DFT calculations. A significant degree of conjugation between the $\pi$-donating bis(pyrrolo)tetrathiafulvalene (BPTTF) part and the electron-withdrawing coordinating pyridyl units is observed. The tetrapyridyl ligand is able to self-assemble in presence of cis-protected $\mathrm{Pt}(\mathrm{II})$ complexes, to produce the first examples of BPTTF-based cages endowed with a cavity prone to guest encapsulation. These metalla-cages undergo an original chemical conversion upon heating in DMSO, to afford Ptdithiolene complexes which could be characterized by XRD in one case. This reaction could be extended to alternative BPTTF derivatives.

\section{Introduction}

The preparation of molecular cages capable of encapsulating organic guests is subject to intense interest, motivated by potential applications in various fields such as chemical sensing, chemical reactivity in confined space or even molecule transport. ${ }^{1}$ Nevertheless, the synthetic access to three-dimensional receptors through classical step-by-step covalent synthesis is often challenging. In this context, the coordination-driven self-assembly methodology constitutes a fruitful alternative which has led to a wide diversity of metalla-cages over the last decade. ${ }^{2}$ Noteworthy, only few examples of such self-assembled cages involve redoxactive side walls ${ }^{3}$ and, if so, they are mostly built from electrondeficient tripyridyl-triazine-based panels. ${ }^{4}$

In the course of our studies related to the design of electron-rich cavities using the metal-driven methodology, we recently investigated the preparation of polygons incorporating a tetrathiafulvalene (TTF) derivative as the basic constituent of the cavity panels, ${ }^{5}$ as well as the preparation of the first examples of polyhedra made of TTF derivatives. ${ }^{6}$ Given the well-established $\pi$ donating ability of tetrathiafulvalene (TTF) derivatives, a motif which has been widely used in various molecular and supramolecular switchable systems, ${ }^{7}$ the resulting self-assembled discrete structures constitute interesting electronically complementary hosts related to the most common supramolecular

\author{
Laboratoire MOLTECH-Anjou \\ CNRS UMR 6200 \\ Université d'Angers, 2bd Lavoisier, 49045 Angers Cedex, France \\ Fax: (+33) (0)2.41.73.54.05 \\ E-mail: marc.salle@univ-angers.fr \\ http://moltech-anjou.univ-angers.fr/
}

cages made from $\pi$-electron accepting ligands.

On this ground, we report herein a study related the synthesis and the characterization of a new family bis-pyrroloTTF (BPTTF) ligands bearing two (compound 2) or four (compound 4) ${ }^{6 \mathrm{a}}$ coordinating pyridyl groups. The study of their electronic properties in connection with preferable conformations in the gas phase and at the solid state is addressed, as well as the synthesis and the binding properties of two corresponding self-assembled cages $6 \mathbf{6}, \mathbf{6 b}$. Finally, an unexpected evolution from this metalla-cages to metal dithiolene complexes upon heating in DMSO, is described.

\section{Results and Discussion}

\section{Synthesis of ligands 2 and 4}

The bis- and tetra-pyridyl derivatives $\mathbf{2}$ and $\mathbf{4}$ were synthesized (scheme 1) starting from mono-pyrroloTTF (MPTTF) and bispyrroloTTF (BPTTF), two well-established TTF derivatives for which the dithiol rings are respectively fused to one or two pyrrole moieties. ${ }^{8}$ A nucleophilic addition onto activated pyridine is carried out through a mild non-organometallic process. The latter is based on activation of the pyridine ring with triflic anhydride, a method which is known to allow a coupling with nucleophilic species such as functionalized pyrroles. ${ }^{9}$ Reaction between MPTTF $^{8 c}$ and the in situ generated $\mathrm{N}$-triflyl cation leads to the $\mathrm{N}$-triflate intermediate 1 in $86 \%$ yield after purification by $\mathrm{SiO}_{2}$ column chromatography. Similarly, the tetra-substituted analogue $\mathbf{3}$ was synthesized from BPTTF $^{8 c}$ and was obtained in $46 \%$ yield, which also constitutes a good yield when considering that four covalent $\mathrm{C}-\mathrm{C}$ bonds are simultaneously formed along this step. The fairly stable intermediates $\mathbf{1}$ and $\mathbf{3}$ were then aromatized under basic conditions $(t$-BuOK), into the target di- and tetra-topic ligands $\mathbf{2}$ and $\mathbf{4}$. 

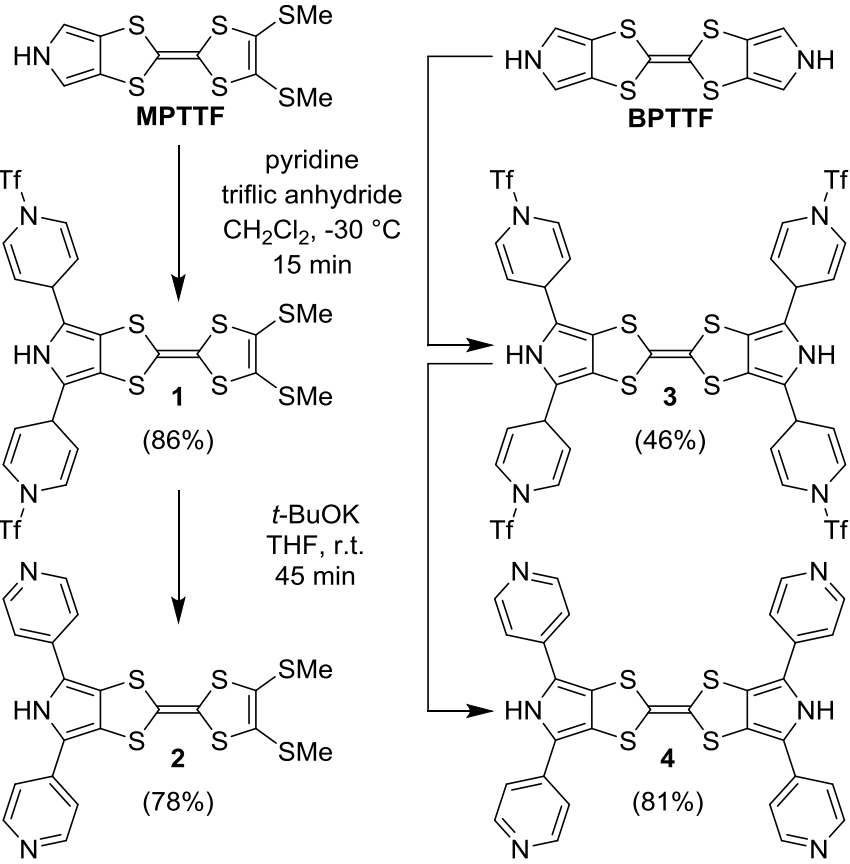

Scheme 1. Synthesis of TTF-based ligands $\mathbf{2}$ and $\mathbf{4}$.

\section{Solid-state structures of the BPTTF-based ligands}

Ligand 4 exhibits a moderate solubility. A much better solubility is found with the corresponding tetrakis(methylpyridinium) BPTTF salt $\mathbf{5}$, which is obtained by permethylation of $\mathbf{4}$ with an excess of methyl iodide in DMF, followed by anion exchange with $\mathrm{KPF}_{6}$ (Scheme 2). A precise knowledge of the structural parameters which characterize a given ligand (including preferable conformations) is particularly helpful to anticipate the spatial organization of the corresponding subsequent self-assemblies. For this purpose, single crystals of ligand $\mathbf{2}$ as well as of the tetrakis(methylpyridinium) derivative 5 were grown. In the first case, slow diffusion of diethylether in a THF solution of $\mathbf{2}$ provides two types of crystals, red and yellow respectively, suitable for X-Ray diffraction studies (XRD) (Table 1). Two distinctive compositions were found for these

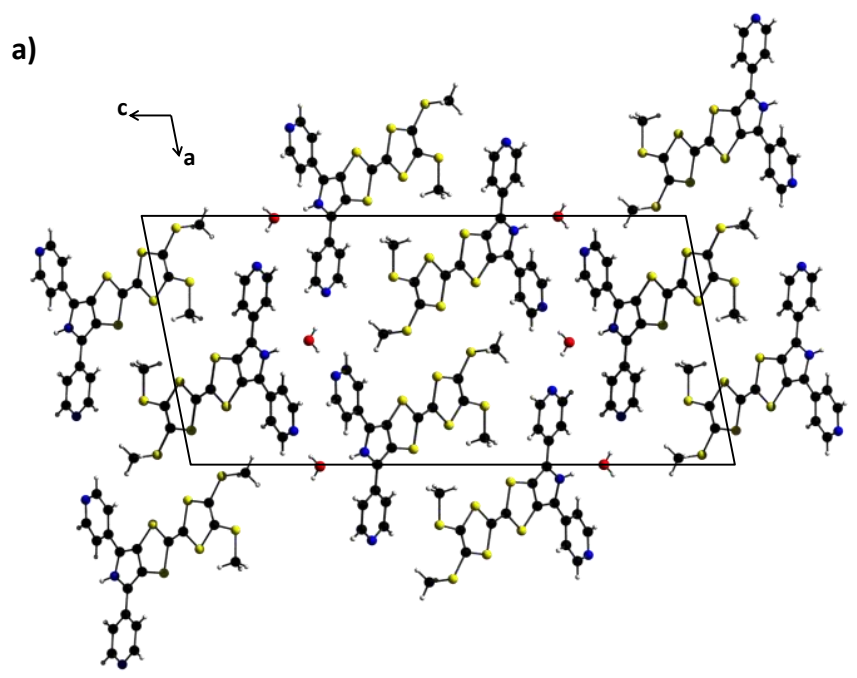<smiles>C1=NC(=C2Sc3c(-c4ccncc4)[nH]c(-c4ccncc4)c3S2)Sc2c1[nH]c(-c1ccncc1)c2-c1ccncc1</smiles>

a) Mel, DMF, $50{ }^{\circ} \mathrm{C}, 4 \mathrm{~h}$ b) $\mathrm{KPF}_{6}, \mathrm{H}_{2} \mathrm{O}$, r.t., $5 \mathrm{~min}$<smiles></smiles>

Scheme 2. Synthesis of compound $\mathbf{5}$.

crystals, one of them incorporating one water molecule per ligand. In the case of $2-\mathrm{H}_{2} \mathrm{O}$, the water molecules form a hydrophilic network along the $a b$ plane, in-between slabs of $\pi$-donating molecules 2 (Figure 1a). The water molecule clearly contributes to the crystallographic packing by establishing three simultaneous $\mathrm{H}$ bonds with three distinctive neighbouring units 2 (Figure 1b). Two of them correspond to interactions with pyridine rings of two different $\pi$-donors 2 (OH(water)... $\mathrm{N}$ (pyridine)), ranging from $2.00(6)$ to $2.10(4) \AA$, the third one being based on a NH...O(water) interaction (1.96(3) $\AA$ ) with the pyrrole moiety of a third unit $\mathbf{2}$. In this structure, the MPTTF skeleton appears essentially planar (Figures 1 and S29). In particular, only a slight deviation from planarity is observed through the $\mathrm{S} \ldots \mathrm{S}$ axis for each 1,3-dithiole rings $\left(\theta=3.04(1)^{\circ}\right.$ and $\left.5.11(2)^{\circ}\right)$ and moderate rotation angles are found around the MPTTF-pyridyl units $\left(9.91(9)^{\circ}\right.$ and $19.96(9)^{\circ}$ respectively), though these bonds can in principle tolerate any rotation angle.

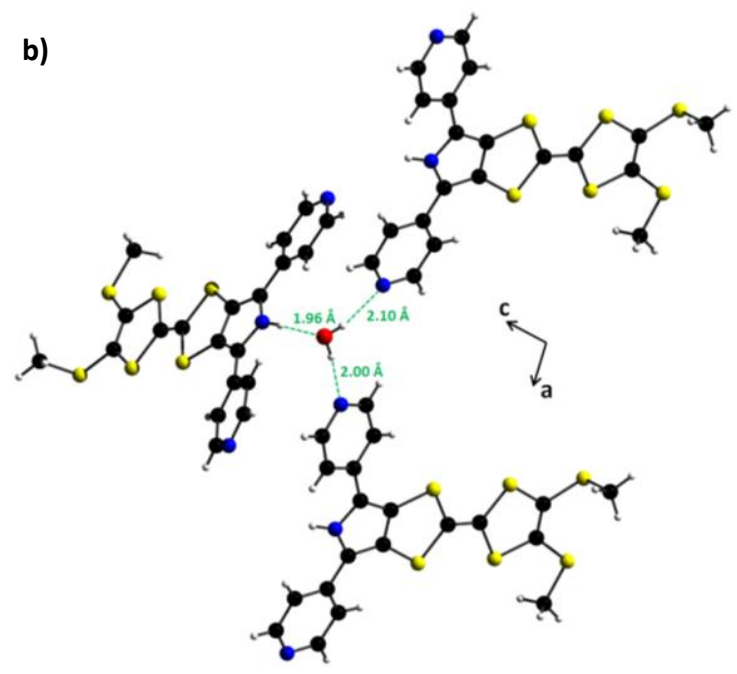

Figure 1. X-ray crystal structure of $\mathbf{2}-\mathrm{H}_{2} \mathrm{O}$, a) view along the ac plane; b) H-bonds network around one water molecule. 
Table 1 Crystallographic data for compounds $2,2 . \mathrm{H}_{2} \mathrm{O}, \mathbf{5}, \mathbf{6 a}$ and $\mathbf{7 b}$.

\begin{tabular}{|c|c|c|c|c|c|c|}
\hline \multicolumn{2}{|c|}{ Compound } & 2. $\mathrm{H}_{2} \mathrm{O}$ & 2 & 5 & $6 \mathbf{a}$ & $7 b$ \\
\hline \multicolumn{2}{|c|}{ empirical formula } & $\mathrm{C}_{20} \mathrm{H}_{17} \mathrm{~N}_{3} \mathrm{OS}_{6}$ & $\mathrm{C}_{20} \mathrm{H}_{15} \mathrm{~N}_{3} \mathrm{~S}_{6}$ & $\mathrm{C}_{46} \mathrm{H}_{58} \mathrm{~F}_{24} \mathrm{~N}_{10} \mathrm{O}_{4} \mathrm{P}_{4} \mathrm{~S}_{4}$ & $\mathrm{C}_{162} \mathrm{H}_{234} \mathrm{~N}_{18} \mathrm{O}_{75} \mathrm{P}_{12} \mathrm{Pt}_{6} \mathrm{~S}_{12}$ & $\mathrm{C}_{44} \mathrm{H}_{39} \mathrm{Cl}_{2} \mathrm{~F}_{6} \mathrm{~N}_{3} \mathrm{O}_{6} \mathrm{P}_{2} \mathrm{PtS}_{4}$ \\
\hline \multicolumn{2}{|c|}{ formula weight } & 507.73 & 489.71 & 1523.14 & 5560.57 & 1275.95 \\
\hline \multicolumn{2}{|c|}{ temperature $(\mathrm{K})$} & 293(2) & 293(2) & $200(2)$ & $200(2)$ & $200(2)$ \\
\hline \multirow{2}{*}{\multicolumn{2}{|c|}{$\begin{array}{l}\text { crystal description and color } \\
\text { crystal system }\end{array}$}} & red needle & yellow plate & black needle & red prism & red prism \\
\hline & & monoclinic & monoclinic & monoclinic & orthorhombic & triclinic \\
\hline \multicolumn{2}{|c|}{ space group } & $P 2_{1} / n$ & $P 2_{1} / c$ & $P 2_{1} / n$ & Pnma & $P-1$ \\
\hline \multirow{6}{*}{$\begin{array}{l}\text { unit cell } \\
\text { dimensions }\end{array}$} & $\mathbf{a}(\AA)$ & $14.015(1)$ & $12.800(2)$ & $6.3094(5)$ & $41.62(1)$ & $13.222(2)$ \\
\hline & b $(\AA)$ & $5.4489(5)$ & $9.896(1)$ & $19.889(1)$ & $34.203(4)$ & $13.956(2)$ \\
\hline & c $(\AA)$ & $30.219(2)$ & $17.622(3)$ & $25.432(1)$ & 19.61(1) & $14.574(2)$ \\
\hline & $\alpha$ & 90 & 90 & 90 & 90 & $82.95(1)$ \\
\hline & $\beta$ & $104.480(7)$ & $92.50(2)$ & $96.084(8)$ & 90 & $85.70(1)$ \\
\hline & $\gamma$ & 90 & 90 & 90 & 90 & $70.87(1)$ \\
\hline \multicolumn{2}{|c|}{$\mathbf{V}\left(\AA^{3}\right)$} & $2269.2(3)$ & $2230.0(6)$ & $3173.4(3)$ & 27915(16) & $2519.8(6)$ \\
\hline \multicolumn{2}{|c|}{$\mathbf{Z}$} & 4 & 4 & 2 & 4 & 2 \\
\hline \multicolumn{2}{|c|}{ calculated density $\left(\mathrm{g} / \mathrm{cm}^{3}\right)$} & 1.486 & 1.459 & 1.594 & 1.323 & 1.682 \\
\hline \multicolumn{2}{|c|}{ absorption coefficient $(\mathrm{MoK} \alpha)\left(\mathrm{mm}^{-1}\right)$} & 0.621 & 0.626 & 0.372 & 3.223 & 3.190 \\
\hline \multicolumn{2}{|c|}{ collected reflections } & 23470 & 17044 & 24250 & 99343 & 37355 \\
\hline \multicolumn{2}{|c|}{ unique reflections, $\mathbf{R}_{\text {int }}$} & $5061,0.075$ & $4340,0.113$ & $6000,0.097$ & $22102,0.201$ & $11428,0.106$ \\
\hline \multicolumn{2}{|c|}{ parameters } & 339 & 268 & 421 & 680 & 625 \\
\hline \multicolumn{2}{|c|}{$\mathrm{R} 1(\mathrm{I}>\mathbf{2 \sigma}(\mathrm{I}))$} & 0.0556 & 0.0614 & 0.0882 & 0.1862 & 0.0679 \\
\hline \multicolumn{2}{|c|}{$\mathrm{wR}(\mathrm{I}>2 \sigma(\mathrm{I}))$} & 0.0925 & 0.1419 & 0.1745 & 0.4577 & 0.1251 \\
\hline \multicolumn{2}{|c|}{ R1 (all data) } & 0.1213 & 0.2089 & 0.2037 & 0.3974 & 0.1251 \\
\hline \multicolumn{2}{|c|}{ wR (all data) } & 0.1106 & 0.2029 & 0.2292 & 0.5863 & 0.1511 \\
\hline \multicolumn{2}{|c|}{ G.O.F $\left(\mathbf{F}^{2}\right)$} & 1.015 & 0.792 & 1.025 & 1.927 & 1.110 \\
\hline \multicolumn{2}{|c|}{ CCDC number } & 928888 & 928887 & 928889 & 909918 & 928890 \\
\hline
\end{tabular}

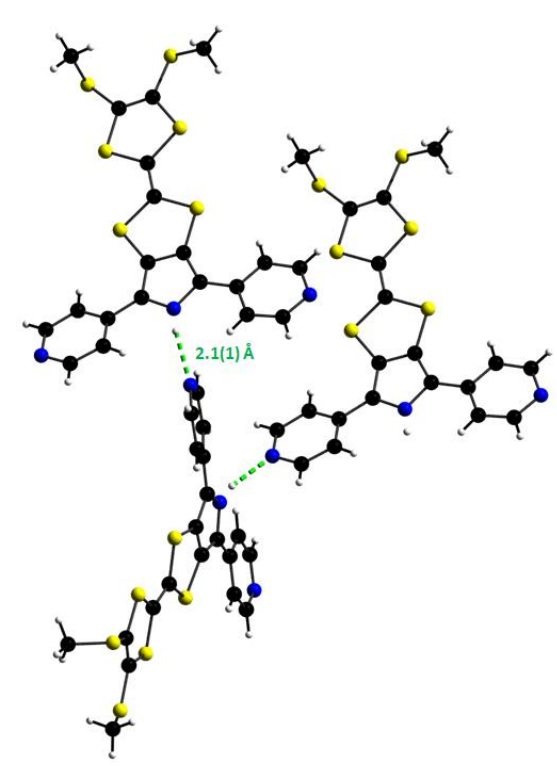

Figure 2. H-bonds network in 2 (X-ray structure).

A distinctive feature is observed regarding the packing mode of the second type of crystals, which corresponds to ligand $\mathbf{2}$ alone. In absence of a water molecule, the crystal organization is in this case governed by an intermolecular H-bonds network involving the N-H pyrrole bond of one ligand $\mathbf{2}$ and one Npyridi atom of a neighbouring ligand $(\mathrm{d}(\mathrm{N}($ pyridi)...HN(pyrrole $)=2.1(1) \AA)$ ) (Figure 2 ). The molecular structure of $\mathbf{2}$ appears very similar to the one observed in $2-\mathrm{H}_{2} \mathrm{O}$, with a very slight torsion of the MPTTF core $\left(1.67(7)^{\circ}\right.$ and $7.97(6)^{\circ}$ related to the central $\mathrm{C}_{2} \mathrm{~S}_{4}$ plane) and rotation angles of $13.5(2)^{\circ}$ and $20.4(2)^{\circ}$ respectively around the MPTTFPyridine bonds (Figure S28).

The molecular structure of the tetrakis(methylpyridinium) BPTTF derivative $\mathbf{5}$ is of striking interest as a model to program the subsequent metal-pyridine self-assembling procedure. Single crystals of $\mathbf{5}$ could be obtained by slow diffusion of diethylether in a $\mathrm{N}, \mathrm{N}$-dimethylformamide solution of the hexafluorophosphate salt. The XRD analysis was carried out and shows that solvent molecules (N,N-DMF) are included within the structure and play a role in the crystal organization through formation of $\mathrm{H}$-bonds with the $\mathrm{N}-\mathrm{H}$ group of the pyrrole moiety $\left(\mathrm{d}\left(\mathrm{O}_{(\mathrm{DMF})}-\mathrm{HN}_{(\text {pyrrole })}\right)=1.980(5) \AA\right.$; Figure S31). All eight heterocycles of 5 are essentially coplanar and the molecules are stacked as dimers along the a axis with short intradimer distances of $3.7 \AA$ (Figure 3 and S26).

It is worth noting that rotation angles around the BPTTFpyridine bonds are even lower in $\mathbf{5}\left(6^{\circ}\right.$ to $\left.8^{\circ}\right)$ than in the case of $\mathbf{2}$ or 2. $\mathrm{H}_{2} \mathrm{O}$. Such observation provides a useful indication about the high degree of intramolecular electronic delocalization between the central $\pi$-donating BPTTF moiety and the four peripheral pyridinium accepting units in $\mathbf{5}$. It also shows that in addition to the electron-withdrawing character of the N-heterocycles in $\mathbf{2 , 4}$ and $\mathbf{5}$, the coplanarity of these units with the $\pi$-donating TTF platform contributes to decrease the $\pi$-donating ability of the system. This could be confirmed in the gas phase, through calculations led on 2,4 and $\mathbf{5}$ (vide infra).

The size and the coordination angles of ligand $\mathbf{4}$, which is designed as a candidate to generate metal-driven self-assembled discrete structures, constitute additional important issues to address. 

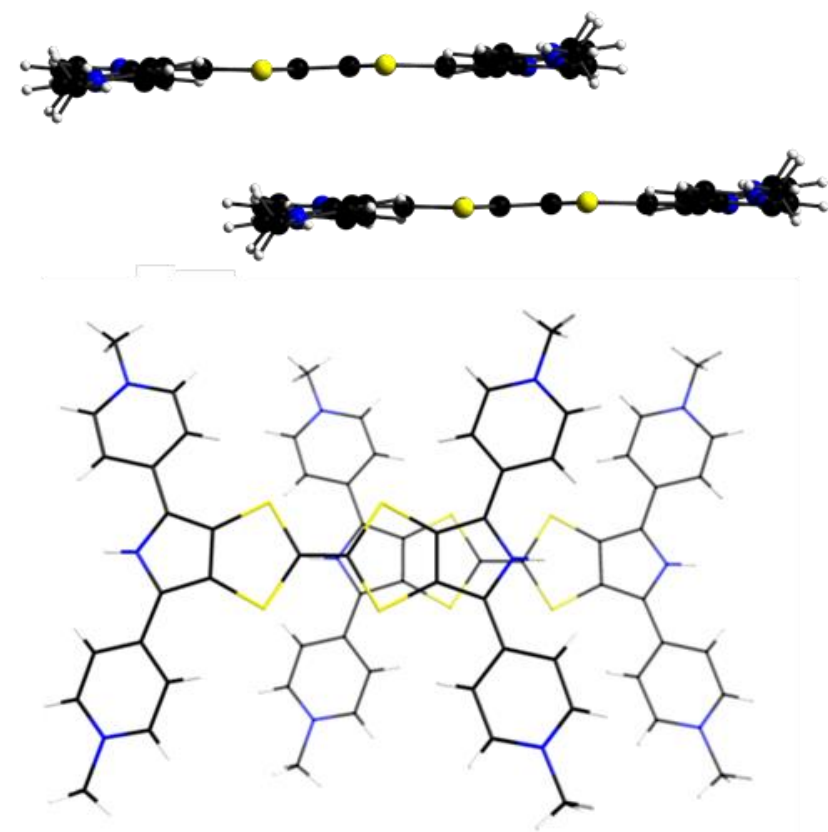

Figure 3. X-ray crystal structure of 5: stacking mode of the tetrasubstituted BPTTF units (solvent molecules and anions omitted for clarity).

Indeed, these metrical parameters govern the size and the shape of the targeted self-assemblies. The four Npyri atoms in $\mathbf{5}$ define a rectangle of $10.18 \times 11.83 \AA$ (Figure S32), which therefore correspond to the size of the lateral panels within the corresponding subsequent self-assembled cavity. On the other hand, coordination angles of $41^{\circ}$ and $140^{\circ}$ are found respectively between two adjacent pyridine units in 5 (Figure S32). These geometrical parameters provide useful indication to anticipate the choice of suitable complementary counter-parts (metal complexes) to be used in the forthcoming self-assembly process.

\section{Theoretical calculations on the BPTTF-based ligands}

Theoretical calculations based on density functional theory (DFT) methods have been performed for both ligands $\mathbf{2}$ and $\mathbf{4}$ with the Gaussian 09 program. ${ }^{10}$ Becke's three-parameter gradient-corrected functional (B3LYP) with 6-31G $(\mathrm{d}, \mathrm{p})$ basis in vacuum was used for full geometry optimization and to compute the electronic structure at the minima found. Figure 4 sketches the atomic orbital composition calculated for the highest occupied (HOMO) and lowest unoccupied (LUMO) molecular orbitals of $\mathbf{2}$ and $\mathbf{4}$ as well as for BPTTF for comparison. In all cases, the HOMO orbital appears essentially centred on the MPTTF part (respectively BPTTF) of the ligands, whereas the corresponding LUMO are localized on the lateral pyridine rings and the outer pyrrole ring. Incorporation of two and four pyridyl fragments for $\mathbf{2}$ and $\mathbf{4}$ strongly impacts the electronic properties of the ligands. The higher HOMO energy of BPTTF ($4.48 \mathrm{eV})$ related to $4(-5.21 \mathrm{eV})$ and $2(-5.03 \mathrm{eV})$ illustrates the progressive decreasing of the $\pi$-donating ability of the BPTTF-based system once two (compound 2) and four (compound 4) pyridyl units are introduced. This observation confirms the high degree of electronic delocalization between the donating BPTTF part and the accepting pyridine units and is in good accordance with observations made above by XRD in the solid state.

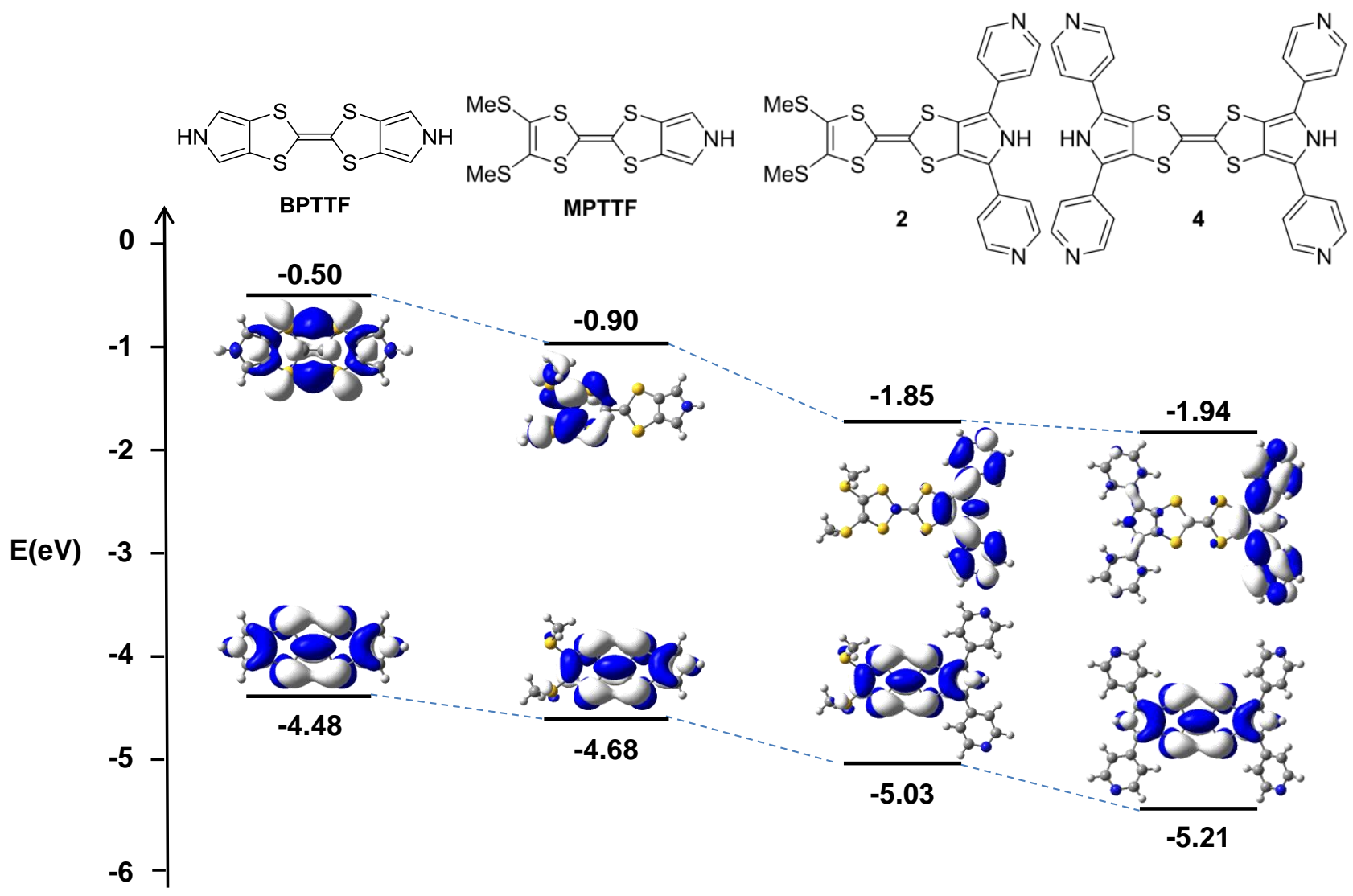

Figure 4. HOMO and LUMO orbitals of reference donors BPTTF, MPTTF and of ligands $\mathbf{2 , 4}$. 


\section{Coordination-driven self-assemblies}

The tetra-pyridyl-BPTTF ligand $\mathbf{4}$ was engaged in self-assembly processes with cis-protected $\mathrm{Pt}(\mathrm{II})$ complexes $\left(\right.$ cis- $\left(\mathrm{Et}_{3} \mathrm{P}\right)_{2} \mathrm{Pt}(\mathrm{OTf})_{2}$ and (dppp) $\left.\mathrm{Pt}(\mathrm{OTf})_{2}\right)$. The course of the self-assembling process was monitored by ${ }^{1} \mathrm{H}-\mathrm{NMR}$. Remarkably, the reaction of $\mathbf{4}$ with a twofold excess of cis- $\left(\mathrm{Et}_{3} \mathrm{P}\right)_{2} \mathrm{Pt}(\mathrm{OTf})_{2}$ or (dppp) $\mathrm{Pt}(\mathrm{OTf})_{2}$ in DMSO at $75^{\circ} \mathrm{C}$, converges within two hours (6a) (respectively twelve hours (6b)), to a single compound, as shown by ${ }^{1} \mathrm{H}-\mathrm{NMR}$ monitoring (Figure 5). The corresponding spectrum tends to a set of well-defined signals, a manifestation of the high symmetry of the resulting product. The $\mathrm{H} \alpha$ and $\mathrm{H} \beta$ protons of the pyridyl units are of course strongly affected upon the coordination process. It is worth noting that in addition to the significant shift upon $\mathrm{Pt}$ coordination, a splitting of both $\mathrm{H} \alpha$ and $\mathrm{H} \beta$ signals is observed for the two complexes $\mathbf{6 a}$ and $\mathbf{6 b}$ when compared to ligand $\mathbf{4}$ (Figure $5 b-d)$. Such observation is assigned to a lack of rotation freedom around the Pt-N bond in the self-assembled structure, which results in $\mathrm{H} \alpha$ (respectively $\mathrm{H} \beta$ ) with different spatial environments. ${ }^{11}$ Formation of only one self-assembled discrete species is confirmed by ${ }^{1} \mathrm{H}$-DOSY NMR spectra of $\mathbf{6 a}$ and $\mathbf{6} \mathbf{b}$ which show only one alignment of signals (Figure 5a,e). This result is also confirmed by the corresponding ${ }^{31} \mathrm{P}$ and ${ }^{19} \mathrm{~F}$ NMR spectra which exhibit only one signal (Figure S15 and S18). In addition, it is worth noting that diffusion coefficients provided by ${ }^{1} \mathrm{H}$-DOSY NMR are identical for both discrete structures $6 \mathbf{a}$ and $\mathbf{6 b}$, which signifies that they are of similar sizes. At this stage, since the self-assembly process is led from a rectangular tetratopic ligand and two equiv. of a $90^{\circ}$ linker, various discrete geometries can be in principle expected, ie trigonal, tetragonal or eventually larger prismatic homologues. ${ }^{11}$ Assemblies $\mathbf{6 a}$ and $\mathbf{6 b}$ were determined to correspond to trigonal prismatic structures as schematized in scheme 3 . A first evidence came from the above NMR experiments, for which the splitting of the ${ }^{1} \mathrm{Hpyr}$

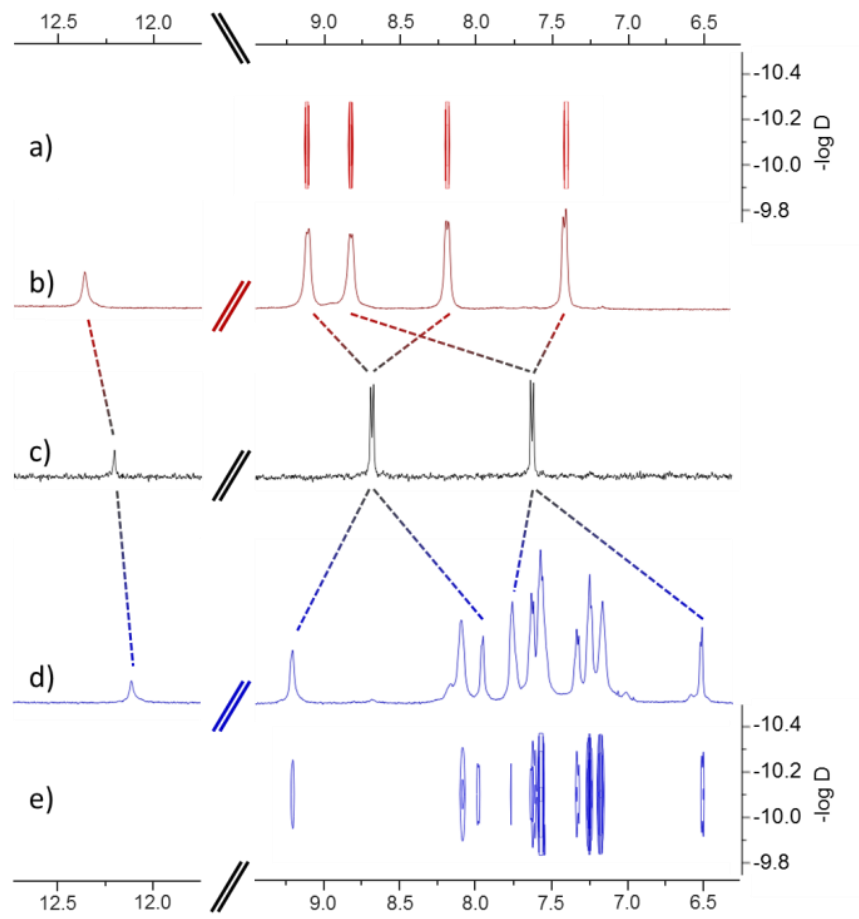

Figure 5. NMR spectra (solvent DMSO- $\mathrm{d}_{6}$ ): downfield region of the a) ${ }^{1} \mathrm{H}$ DOSY NMR of $\mathbf{6 a}$, b) ${ }^{1} \mathrm{H}$ NMR spectrum of $\left.\mathbf{6 a}, \mathrm{c}\right){ }^{1} \mathrm{H}$ NMR spectrum of ligand 4, d) ${ }^{1} \mathrm{H}$ NMR spectrum of $\left.\mathbf{6 b}, \mathrm{e}\right){ }^{1} \mathrm{H}$ DOSY NMR of $\mathbf{6 b}$.

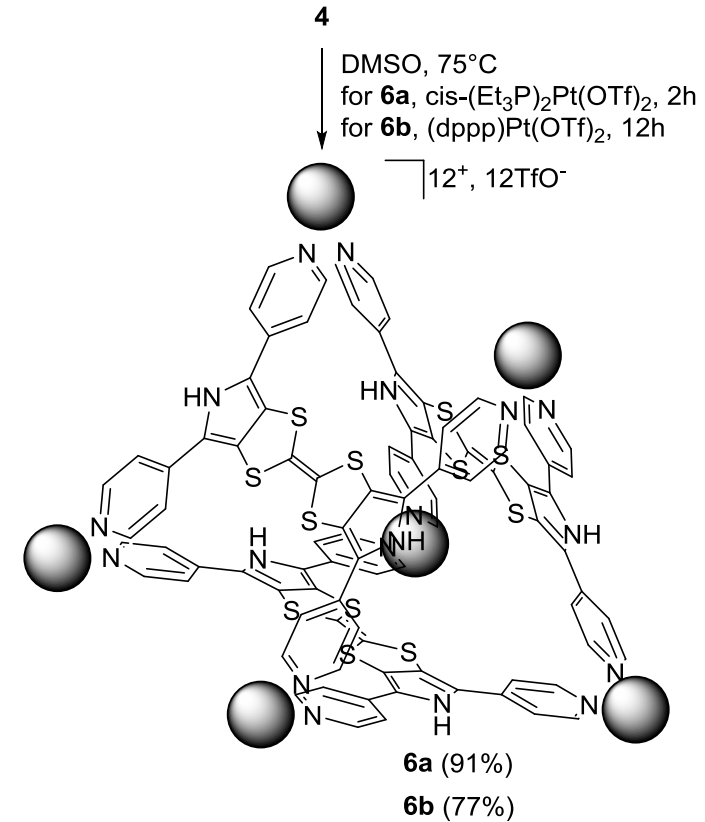

Scheme 3. Synthesis of the trigonal prisms $\mathbf{6 a}$ and $\mathbf{6 b}$.

signals characterizes a restricted rotation around the BPTTFpyridine bond, giving rise to protons with different magnetic environment (inside and outside the cavity). This feature corresponds to a known behavior for trigonal prismatic structures. ${ }^{11}$ The latter geometry was confirmed by ESI mass spectrometry analyses led on $\mathbf{6 a}$, from which peaks were observed at $\mathrm{m} / \mathrm{z} 1388.4$, $1080.7,875.6$, and 729.1 which correspond to the progressive loss of triflate $\left(\mathrm{TfO}^{-}\right)$counterions $[\mathbf{6 a}-4 \mathrm{OTf}]^{4+},[\mathbf{6} \mathbf{a}-5 \mathrm{TfO}]^{5+},[\mathbf{6} \mathbf{a}-$ $6 \mathrm{TfO}^{6+}$ and $[\mathbf{6 a}-7 \mathrm{TfO}]^{7+}$. In the case of $\mathbf{6 b}$, a similar distribution was found at $\mathrm{m} / \mathrm{z}=1231.92,1201.92,1001.77$ and 976.78 which correspond to multicharged species $\left[6 \mathbf{b}-2 \mathrm{H}^{+}-7 \mathrm{OTf}\right]^{5+},\left[6 \mathbf{b}-3 \mathrm{H}^{+}-\right.$ $8 \mathrm{OTf}]^{5+},\left[6 \mathbf{6 b}-2 \mathrm{H}^{+}-8 \mathrm{OTf}\right]^{6+}$ and $\left[6 \mathbf{6 b}-3 \mathrm{H}^{+}-9 \mathrm{OTf}\right]^{6+}$. Experimental isotopic patterns centred on these $\mathrm{m} / \mathrm{z}$ values suitably correlate with the corresponding theoretical isotopic distribution (Figure S25 and S26). Finally, the trigonal prismatic geometry was definitely consolidated by an XRD study led on $\mathbf{6 a}^{6 \mathrm{a}}$ from single crystals grown by slow diffusion of diethyl ether in an acetonitrile solution of the complex (Figure 6). Noteworthy, as anticipated from the above calculations led on $\mathbf{4}$ and $\mathbf{5}$, all pyridyl rings are essentially coplanar with the BPTTF skeleton within the complex. Consequently, a regular cavity of 8-9 $\AA$ inner diameter is formed. The BPTTF skeletons constituting the panels of the cavity appear slightly distorted $\left(12^{\circ}\right.$ related to the $\mathrm{C}_{2} \mathrm{~S}_{4}$ central BPTTF skeleton). This torsion partly compensates the geometric constraint generated from a cis-protected square planar complex, for which a theoretical $90^{\circ}$ coordination angle is awaited. Such a bending of the organic bridge between two metals has already been observed for selfassemblies involving long linear ligands, ${ }^{12}$ and allows to decompress the coordination angle on the metal. Nevertheless, one can note that the angles around the metal in $\mathbf{6 a}$ are still low (NpyrPt-Npyr $=78-84^{\circ}$ ) regarding the usual square-planar coordination mode of Pt(II). 


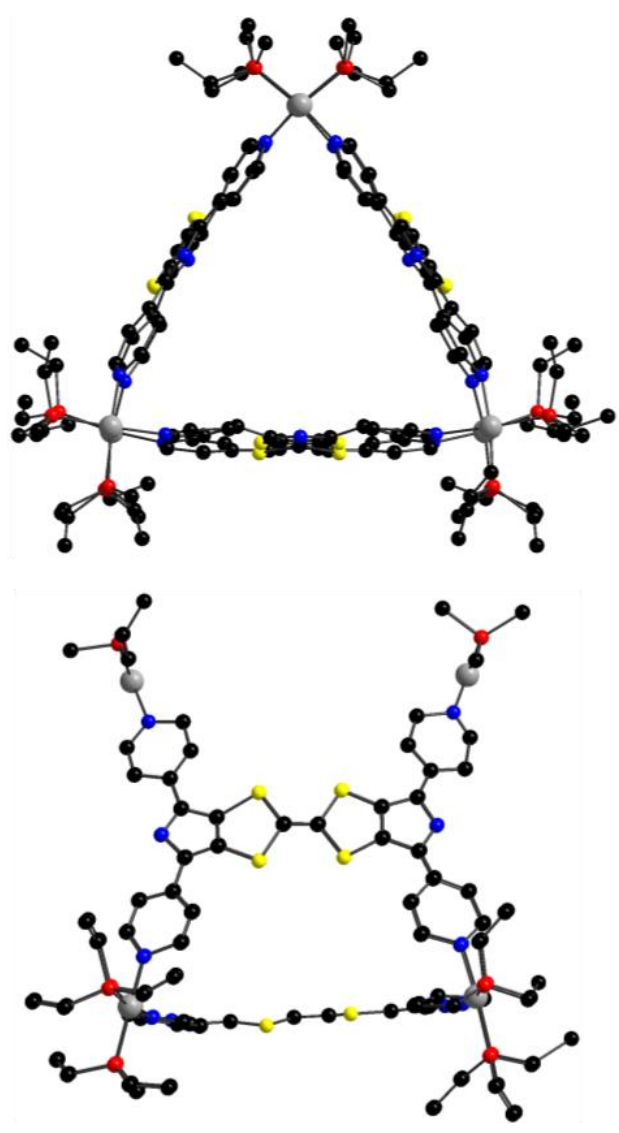

Figure 6. X-Ray crystal structure of cage $\mathbf{6 a}$ (anions and $\mathrm{H}$ atoms of ethyl groups omitted)

\section{Electronic properties}

Electrochemical properties of the self-assembled cage 6a were studied by cyclic voltammetry in acetonitrile $\left(n \mathrm{Bu}_{4} \mathrm{NPF}_{6}, 0.1\right.$ mol. $\mathrm{L}^{-1}$ ) and were compared with those of the soluble tetracationic salt 5. ${ }^{13}$ As seen from Figure 7, the tetrakis(methylpyridinium) derivative $\mathbf{5}$ and the cage $\mathbf{6 a}$ display a similar $\pi$-donating ability (0.4-0.5 V vs $\left.\mathrm{Fc} / \mathrm{Fc}^{+}\right)$, and are both oxidized at much higher

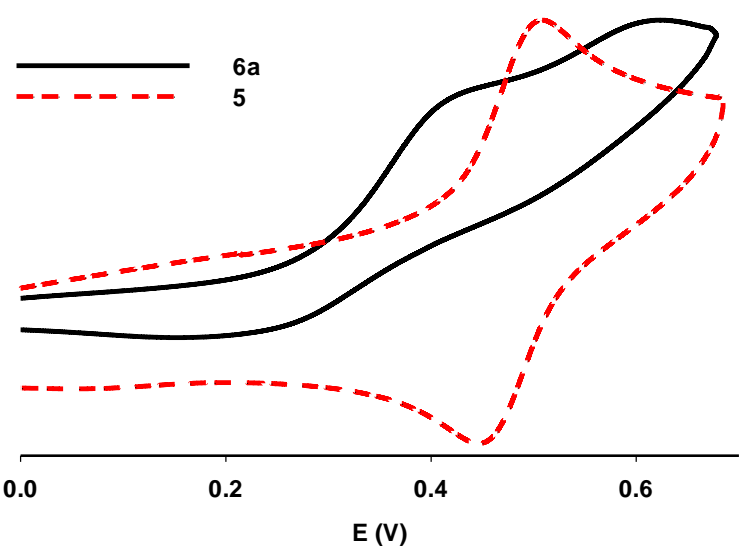

Figure 7. Cyclic voltammogram of $6 \mathbf{a}\left(\mathrm{C}=6 \times 10^{-4} \mathrm{M}\right)$ and $\mathbf{5}(\mathrm{C}=6 \times 10$

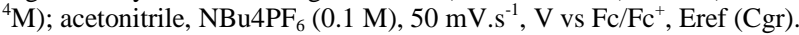

potentials than pristine BPTTF $\left(\mathrm{E}_{1}{ }^{\text {ox }}=-0.02 \mathrm{~V} ; \mathrm{E}_{2}{ }^{\text {ox }}=0.23 \mathrm{~V}\right.$, Figure S27). This result accounts for the electro-withdrawing effect generated by the four pyridinium moieties (case of $\mathbf{5}$ ), or once the metal coordination is achieved (case of $\mathbf{6 a}$ ). As anticipated from XRD and geometrical optimization (vide supra), such observations are fully coherent with an electronic conjugation between the donating and accepting parts of the ligand.

The UV-vis spectroscopic analyses of $\mathbf{5}$ and $\mathbf{6 a}$ (Figure 8) were carried out in acetonitrile and are consistent with $\mathrm{CV}$ observations. Both absorption spectra exhibit evident similarities, which confirms that compound $\mathbf{5}$ constitutes a pertinent model for comparison with the metal-coordinated assembly 6a. Both compounds exhibit two bands (5: $374 \mathrm{~nm}$ and $473 \mathrm{~nm}$; $6 \mathbf{6}: 355 \mathrm{~nm}$ and $442 \mathrm{~nm}$ ). The high-energy one is ascribed to a transition centred on the BPTTF framework ${ }^{14}$ whereas the second one is dominated by an intramolecular charge transfer (ICT) between the electron-rich BPTTF unit and the pyridine accepting-fragments, be they methylated (5) or engaged in the metal coordination $(\mathbf{6 a})$. The high $\varepsilon$ values observed for this band (5: $\varepsilon=55000 \mathrm{~L} \cdot \mathrm{mol}^{-1} \cdot \mathrm{cm}^{-1}$; 6a: $\varepsilon=105500 \mathrm{~L} \cdot \mathrm{mol}^{-1} \cdot \mathrm{cm}^{-1}$ ) is attributed to the cumulated effect of the four electron-accepting pyridine groups connected to the BPTTF donating framework.

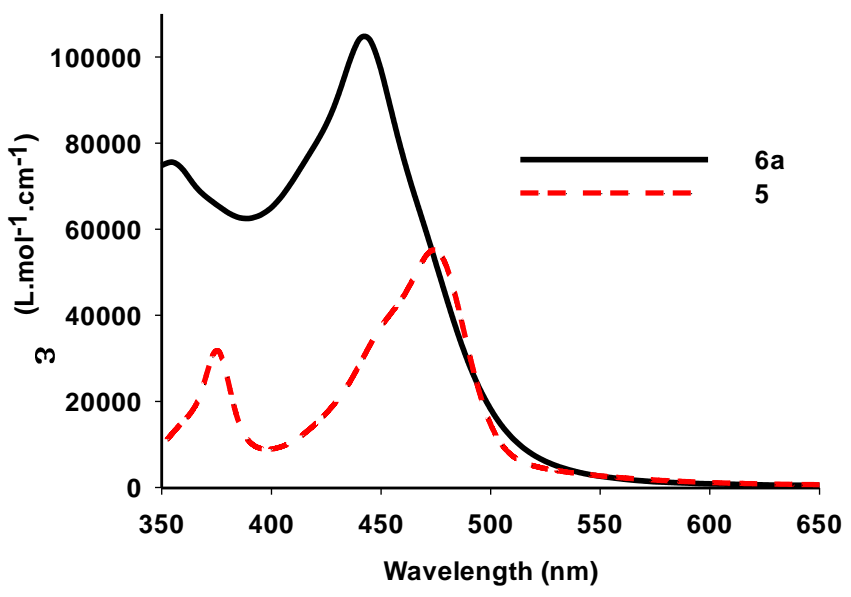

Figure 8. UV-vis spectra of compounds $6 \mathbf{a}\left(\mathrm{C}=6 \times 10^{-6} \mathrm{M}\right)$ and $\mathbf{5}(\mathrm{C}=1.3$ $\left.\times 10^{-5} \mathrm{M}\right)$, acetonitrile.

We recently demonstrate by a UV-vis titration study that prismatic cage 6a is able to include one $\mathrm{TCNQF}_{4}$ molecule. ${ }^{6 a}$ In addition to be a complementary electron-poor system, the latter presents a suitable size and geometry for inclusion within cage $\mathbf{6 a}$ To the best of our knowledge, this corresponds to the first example of inclusion within a TTF-based self-assembly and opens promising perspectives for the inclusion control of an electron-poor guest within a cavity.

\section{A synthetic access to functionalized dithiolenes}

The formation of prismatic structures $\mathbf{6 a}$ and $\mathbf{6 b}$ are mainly governed by entropic factors. In an attempt to reach self-assembled prismatic higher homologues, eg tetragonal prisms, we studied the self-assembly process between $\mathbf{4}$ and the Pt(II) complexes (cis$\left(\mathrm{Et}_{3} \mathrm{P}\right)_{2} \mathrm{Pt}(\mathrm{OTf})_{2}$ and $\left.(\mathrm{dppp}) \mathrm{Pt}(\mathrm{OTf})_{2}\right)$.at different concentrations in DMSO. Whereas no noticeable effect could be observed from these experiments, we observed that a prolonged heating of those 


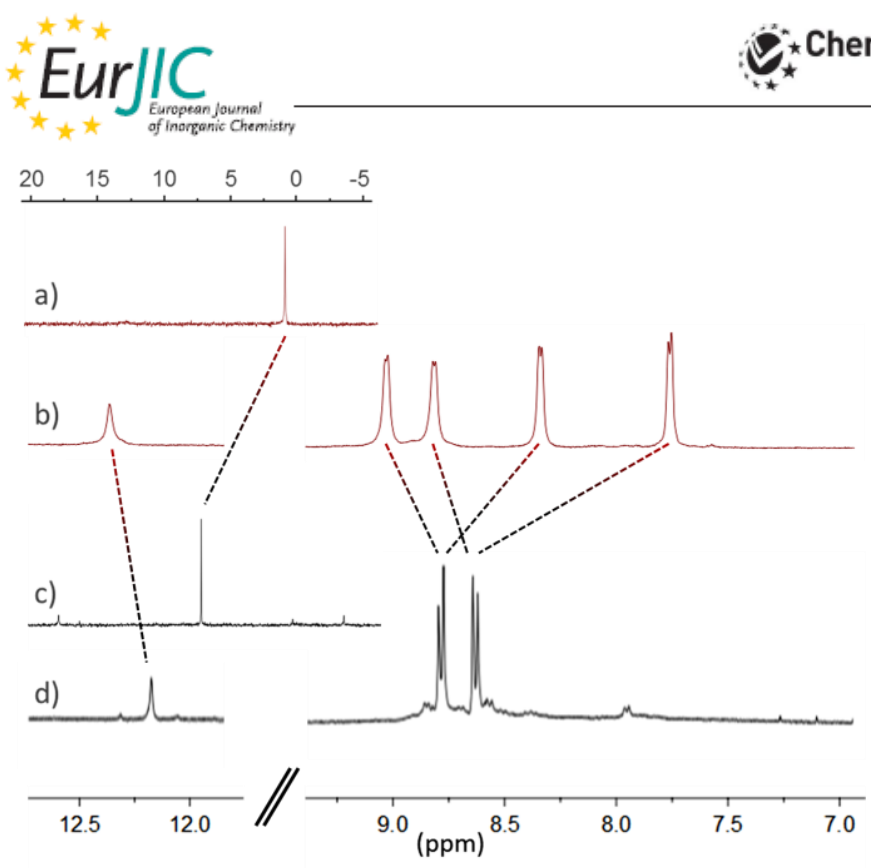

Figure 9. ${ }^{31} \mathrm{P}$ NMR (a) and ${ }^{1} \mathrm{H}$ NMR (b) spectra of $6 \mathbf{a}$ in DMSO-d6; evolution after 7 days at $75^{\circ} \mathrm{C}$ : c) ${ }^{31} \mathrm{P}$ and d) ${ }^{1} \mathrm{H}$ spectra of $7 \mathbf{a}$.

solutions (several days) resulted in an interesting chemical evolution. A typical case is shown in Figure 9, where it is seen by ${ }^{1} \mathrm{H}-\mathrm{NMR}$ that a new definite compound $\mathbf{7 a}$ is formed quantitatively with time, from self-assembly $\mathbf{6 a}$ (scheme 4). Contrariwise to $\mathbf{6 a}$, compound 7a does not exhibit a split of the ${ }^{1}$ Hpyr signals, which indicates that pyridine moieties are in this case able to rotate freely. In addition, the ${ }^{31} \mathrm{P}$ spectrum confirms occurrence of a single product (Figure S21) and the diffusion coefficient extracted from the DOSY-NMR shows that the size of the latter is significantly smaller than starting derivative 6a (Figure S22). The same behavior was observed upon heating the trigonal prismatic structure $\mathbf{6 b}$, which gives rise to $\mathbf{7 b}$. Single crystals of $\mathbf{7 b}$ could be grown by slow diffusion of diethyl ether in a dichloromethane solution and were analyzed by XRD (Figure 10 and Table 1). This study indicates that structure $\mathbf{7}$ actually corresponds to a dithiolene framework.

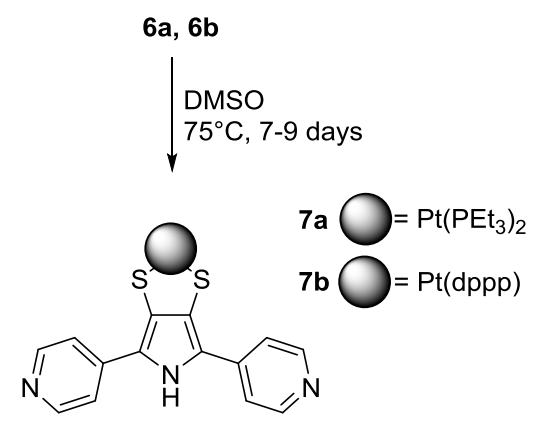

Scheme 4. Synthesis of heteroleptic dithiolenePt complexes $\mathbf{7 a}, \mathbf{b}$

By contrast with homoleptic dithiolene complexes, only a few heteroleptic complexes are known. ${ }^{15}$ The dithiolene framework in 7b co-crystallizes with one molecule of dichloromethane and two triflates counter anions of the two pyridinium entities. Interestingly, the protonated pyridyl units appear essentially coplanar with the dithiolene moiety in the solid state structure, as was the case of BPTTF analogues $\mathbf{5}$ and $\mathbf{6 a}$, illustrating here-again a good conjugation through the central framework. Such a quantitative
FULL PAPER

chemical conversion from a BPTTF skeleton toward a dithiolene framework was unexpected, though a metal-assisted C-S cleavage in TTF derivatives was recently described. ${ }^{16}$ Based on these observations, we initiated a study starting directly from BPTTF derivatives instead of self-assemblies $\mathbf{6 a}, \mathbf{b}$, by heating DMSO solutions of various substituted BPTTF derivatives at $75{ }^{\circ} \mathrm{C}$ in presence of a $\mathrm{Pt}(\mathrm{II})$ complex. Among others, BPTTF derivatives presented in the present work were tested, ie ligand $\mathbf{4}$, tetracationic hexafluorophosphate salt $\mathbf{5}$, as well as compound $\mathbf{8}$, a precursor in

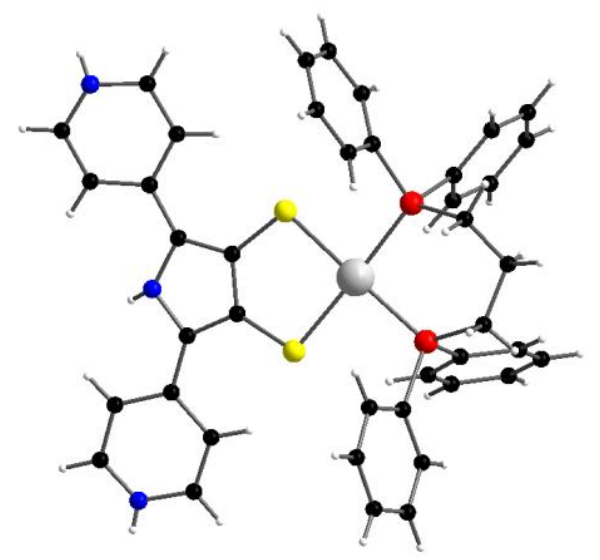

Figure 10. X-Ray solid-state structure of dithiolene complex 7b (two triflate counter anions and one dichloromethane solvent molecule have been omitted for clarity).

the synthesis of starting BPTTF. ${ }^{8 c}$ No reaction was observed in the latter case, whereas evolutions to dithiolene derivatives 7a and 9 were observed from 4 and 5 respectively (scheme S1). A similar test was run in the case of ligand $\mathbf{4}$ in presence of one equivalent of a gold complex $\mathrm{AuBr}_{2} \cdot \mathrm{NBu}_{4}$, a metal known to display a strong affinity for sulfur atoms. Interestingly, the reaction proceeds much faster in this case, since only few hours are needed at room temperature for complete conversion of $\mathbf{4}$ to dithiolene 7a. Several key issues can be drawn from these preliminary data: $i$ ) the cleavage around the $\mathrm{C}_{2} \mathrm{~S}_{4}$ fragment seems favoured in the case of TTF derivatives bearing electron-withdrawing substituents (case of $\mathbf{4}, \mathbf{5}$ ), ii) no coordination of the metal complex is required prior to chemical conversion (case of 5), and iii) the use of a gold metal center favours the reaction, a consequence of the Au-S affinity, which also contributes to decrease the electron density on the sulfur heterocycle. On this basis, this conversion presumably proceeds through insertion of the metallic center within the C-S bond to lead the final dithiolene. Regarding the broad interest of dithiolene derivatives in materials chemistry, ${ }^{17}$ the scope of this reaction in the synthesis of functionalized dithiolene derivatives is under study from alternative substituted TTF derivatives.

\section{Conclusion}

An efficient and straightforward functionalization of the BPTTF core is described, which affords di- and tetratopic ligands 2 and $\mathbf{4}$. These ligands exhibit a high level of intramolecular electronic conjugation. Their self-assembly with Pt(II) complexes afford the first examples of 3D cages incorporating the electron-rich TTF framework. This result opens promising perspectives towards the construction of electron-rich receptors, complementary to the well- 
established electron-poor self-assembled hosts. Finally, an original synthetic conversion from these metalla-assemblies to dithiolene derivatives is depicted.

\section{Experimental Section}

\section{Bis(2,5-di(1-(trifluoromethylsulfonyl)-1,4-dihydro-4pyridyl)[3,4-d]pyrrolo-} tetrathiafulvalene (3): To a solution of pyridine $(389 \mathrm{mg}, 4.91 \mathrm{mmol})$ in anhydrous dichloromethane $(50 \mathrm{~mL})$ at $-30{ }^{\circ} \mathrm{C}$, was added trifluoromethanesulfonic anhydride $(938 \mathrm{mg}, 3.32 \mathrm{mmol})$. After 10 minutes, a white precipitate appeared and a solution of BPTTF $(100 \mathrm{mg}, 0.35 \mathrm{mmol})$ in anhydrous tetrahydrofuran $(5 \mathrm{~mL})$ was added dropwise. The mixture was stirred for $40 \mathrm{~min}$. The reaction was quenched with brine and warmed to room temperature. The organic layer was washed twice with water, dried over magnesium sulfate and concentrated. The residue was purified by chromatography on silica gel, eluting with dichloromethane/Et ${ }_{3} \mathrm{~N}$ (v/v 96/4) to give compound 3 (181 mg, $16 \mathrm{mmol}, 46 \%$ ) as an air sensitive brown solid. $\mathrm{mp}>260{ }^{\circ} \mathrm{C}$ ${ }^{1} \mathrm{H}$ NMR $\left(\left(\mathrm{CD}_{3}\right)_{2} \mathrm{SO}, 25{ }^{\circ} \mathrm{C}\right.$, TMS $) \delta=11.09(\mathrm{~s}, 2 \mathrm{H}), 6.67\left(\mathrm{~d},{ }^{3} \mathrm{~J}=7.4 \mathrm{~Hz}, 8 \mathrm{H}\right), 5.26(\mathrm{dd}$ $\left.{ }^{3} J=7.4 \mathrm{~Hz} \&{ }^{4} J=3.6 \mathrm{~Hz}, 8 \mathrm{H}\right), 4.20$ (brs, $\left.4 \mathrm{H}\right) ;{ }^{13} \mathrm{C}$ NMR $\left(\left(\mathrm{CD}_{3}\right)_{2} \mathrm{SO} ; 25{ }^{\circ} \mathrm{C}\right.$, TMS $)$ $\delta=123.54,121.29\left(\mathrm{q},{ }^{1} J=326.3 \mathrm{~Hz}\right), 120.75,119.07,116.19,110.39,30.71 ;{ }^{19} \mathrm{~F}$ NMR $\left(\left(\mathrm{CD}_{3}\right)_{2} \mathrm{SO}, 25^{\circ} \mathrm{C}, \mathrm{TMS}\right) \delta=-76.93$; IR $(\mathrm{KBr}) 1687,1075 \mathrm{~cm}^{-1}, \mathrm{HRMS}(\mathrm{ESI}) \mathrm{m} / \mathrm{z}$ calcd for $\mathrm{C}_{34} \mathrm{H}_{22} \mathrm{~F}_{12} \mathrm{~N}_{6} \mathrm{O}_{8} \mathrm{~S}_{8}$ : $1125.9072\left[\mathrm{M}^{+}\right]$; found 1125.9073 .

Bis(2,5-di(4-pyridyl)[3,4-d]pyrrolotetrathiafulvalene (4): To a solution of the triflate derivative $3(180 \mathrm{mg}, 0.16 \mathrm{mmol})$ in tetrahydrofuran $(120 \mathrm{~mL})$, was added under argon potassium tert-butoxide $(405 \mathrm{mg}, 3.61 \mathrm{mmol})$ in one portion. The solution was stirred at room temperature for $90 \mathrm{~min}$. Methanol $(20 \mathrm{~mL})$ was poured into the solution and the reaction mixture was stirred for $15 \mathrm{~min}$. Then, the solvent was evaporated until a third and water was added. The red suspension was allowed to stay in the fridge for one night. The resulting solid was centrifuged and washed with water, methanol and dichloromethane to give compound $4(78 \mathrm{mg}, 0.13 \mathrm{mmol}, 81 \%)$ as a red powder. ${ }^{1} \mathrm{H}$ NMR $\left(\left(\mathrm{CD}_{3}\right)_{2} \mathrm{SO}, 25{ }^{\circ} \mathrm{C}\right.$, TMS $) \delta=12.21(\mathrm{~s}, 2 \mathrm{H}), 8.69\left(\mathrm{~d},{ }^{3} \mathrm{~J}=6.1 \mathrm{~Hz}, 8 \mathrm{H}\right)$ $7.60\left(\mathrm{~d},{ }^{3} J=6.1 \mathrm{~Hz}, 8 \mathrm{H}\right)$; HRMS (ESI) $\mathrm{m} / \mathrm{z}$ calcd for $\mathrm{C}_{30} \mathrm{H}_{8} \mathrm{~N}_{6} \mathrm{~S}_{4}: 590.0492\left[\mathrm{M}^{+}\right]$; found 590.0476; element analysis calcd (\%) calcd for $\mathrm{C}_{30} \mathrm{H}_{18} \mathrm{~N}_{6} \mathrm{~S}_{4}+\mathrm{H}_{2} \mathrm{O}$ : C 59.19, H 3.31, N 13.80, S 21.07; found: C 59.09, H 3.17, N 13.59, S 21.55 .

Cage (6a): A mixture of 4 (10.0 mg, $17 \mu \mathrm{mol})$ and cis-Pt(PEt)3(OTf)2 (25.0 mg, 34 $\mu \mathrm{mol})$ in DMSO $(1 \mathrm{~mL})$ was heated at $75^{\circ} \mathrm{C}$ for $2 \mathrm{~h}$ under argon. Then, toluene was added and the mixture was centrifuged. The residue was washed with toluene, diethyl ether and dried under vacuum to give $\mathbf{6 a}(31.9 \mathrm{mg}, 5.2 \mu \mathrm{mol}, 91 \%)$. as a dark red solid. Monocrystals (red, small lozenges) were obtained by slow diffusion of ether in methanol (gaz-liquid). mp $>260{ }^{\circ} \mathrm{C} ;{ }^{1} \mathrm{H}$ NMR $\left(\left(\mathrm{CD}_{3}\right)_{2} \mathrm{SO}, 25{ }^{\circ} \mathrm{C}\right.$, TMS) $\delta=12.39$ (s, $6 \mathrm{H}), 9.06\left(\mathrm{~d},{ }^{3} J=4.4 \mathrm{~Hz}, 12 \mathrm{H}\right), 8.77\left(\mathrm{~d},{ }^{3} J=4.4 \mathrm{~Hz}, 12 \mathrm{H}\right), 8.14\left(\mathrm{~d},{ }^{3} J=4.6 \mathrm{~Hz}, 12 \mathrm{H}\right)$ $7.36\left(\mathrm{~d},{ }^{3} \mathrm{~J}=5.4 \mathrm{~Hz}, 12 \mathrm{H}\right), 1.88(\mathrm{br} s, 72 \mathrm{H}), 1.28-1.17(\mathrm{~m}, 108 \mathrm{H}) ;{ }^{13} \mathrm{C}$ NMR $\left(\left(\mathrm{CD}_{3}\right)_{2} \mathrm{SO}, 25{ }^{\circ} \mathrm{C}, \mathrm{TMS}\right) \delta=150.16,149.88,138.70,123.96,123.30,122.79,120.84$ $120.34,126.95-114.22\left(\mathrm{q}, J_{\mathrm{C}-\mathrm{F}}=320.96 \mathrm{~Hz}\right), 14.59-14.09(\mathrm{~m}), 7.61 ;{ }^{31} \mathrm{P}$ NMR $\left(\left(\mathrm{CD}_{3}\right)_{2} \mathrm{SO}, 25{ }^{\circ} \mathrm{C}\right.$, TMS $) \delta=0.35\left(J_{\mathrm{Pt}-\mathrm{P}}=3067.8 \mathrm{~Hz}\right) ;{ }^{19} \mathrm{~F} \mathrm{NMR}\left(\left(\mathrm{CD}_{3}\right)_{2} \mathrm{SO}, 25^{\circ} \mathrm{C}\right.$, TMS) $\delta=-80.05$; HRMS (ESI-FT-ICR) $\mathrm{m} / \mathrm{z}$ calcd for $\mathrm{C}_{174} \mathrm{H}_{234} \mathrm{~F}_{36} \mathrm{~N}_{18} \mathrm{O}_{36} \mathrm{P}_{12} \mathrm{Pt}_{6} \mathrm{~S}_{24}$ : $875.6222\left([\mathbf{6 a}-6 \mathrm{OTf}]^{6+}\right), 1080.7373\left([\mathbf{6 a}-5 \mathrm{OTf}]^{5+}\right)$; found $875.6203\left([\mathbf{6 a}-6 \mathrm{OTf}]^{6+}\right)$ $1080.7332 \quad\left([\mathbf{6 a}-5 \mathrm{OTf}]^{5+}\right)$; element analysis calcd $(\%)$ calcd for $\mathrm{C}_{174} \mathrm{H}_{234} \mathrm{~F}_{36} \mathrm{~N}_{18} \mathrm{O}_{36} \mathrm{P}_{12} \mathrm{Pt}_{6} \mathrm{~S}_{24}$ : C 33.98, H 3.84, N 4.10, S 12.51, O, 9.37; found: C 33.68, $\mathrm{H} 3.88, \mathrm{~N} 4.10$.

Supporting Information (see footnote on the first page of this article): chemicals and instrumentation, complementary experimental procedures and characterization data, NMR spectra, Mass spectra of $\mathbf{6 a}, \mathbf{6 b}$, Cyclic Voltammetry and X-Ray Structures.

\section{Acknowledgements}

The authors gratefully acknowledge the CNRS and the Région des Pays de la Loire for a $\mathrm{PhD}$ grant (SB), as well as the PIAM (Univ. Angers) and the CRMPO (Univ. Rennes) for their assistance in spectroscopic analyses and finally the Johnson-Matthey company for their generous providing of palladium and platinum salts.

[1] J. W. Steed and J. L. Atwood, Supramolecular chemistry, Wiley, Chichester, UK, 2009

[2] a) K. Harris, D. Fujita, M. Fujita, Chem. Commun. 2013, 49, 67036712 ; b) H. Amouri, C. Desmarets, J. Moussa, Chem. Rev. 2012, 112,
2015-2041; c) L. R. MacGillivray, Angew. Chem. Int. Ed. 2012, 51 , 1110-1112; d) R. Chakrabarty, P. S. Mukherjee, P. J. Stang, Chem Rev. 2011, 111, 6810-6918; e) Y. Inokuma, M. Kawano, M. Fujita, Nat. Chem. 2011, 3, 349-358; f) P. Jin, S. J. Dalgarno, J. L. Atwood, Coord. Chem. Rev. 2010, 254, 1760-1768; g) S. De, K. Mahata, M. Schmittel, Chem. Soc. Rev. 2010,39, 1555-1575; h) B. Therrien, Eur J. Inorg. Chem. 2009, 2445-2453; i) B. H. Northrop, Y. R. Zheng, K. W. Chi, P. J. Stang, Accounts Chem. Res. 2009, 42, 1554-1563; j) P J. Stang, J. Org. Chem. 2009, 74, 2-20; k) M. Yoshizawa, J. K. Klosterman, M. Fujita, Angew. Chem. Int. Ed. 2009, 48, 3418-3438; 1) Y. F. Han, W. G. Jia, W. B. Yu, G. X. Jin, Chem. Soc. Rev. 2009 $38,3419-3434$

[3] a) C. H. M. Amijs, G. P. M. van Klink, G. van Koten, Dalton Trans. 2006, 308-327; b) A. K. Bar, S. Mohapatra, E. Zangrando, P. S Mukherjee, Chem. Eur. J. 2012, 18, 9571-9579; c) N. Fujita, K. Biradha, M. Fujita, S. Sakamoto, K. Yamaguchi, Angew. Chem. Int. Ed. 2001, 40, 1718-1721.

[4] Y. Furutani, H. Kandori, M. Kawano, K. Nakabayashi, M. Yoshizawa, M. Fujita, J. Am. Chem. Soc. 2009, 131, 4764-4768.

[5] a) S. Goeb, S. Bivaud, P. I. Dron, J. Y. Balandier, M. Chas, M. Sallé, Chem. Commun. 2012, 48, 3106-3108; b) J. Y. Balandier, M. Chas, S. Goeb, P. I. Dron, D. Rondeau, A. Belyasmine, N. Gallego, M. Sallé, New J. Chem. 2011, 35, 165-168; c) J. Y. Balandier, M. Chas, P. I. Dron, S. Goeb, D. Canevet, A. Belyasmine, M. Allain, M. Salle, J. Org. Chem. 2010, 75, 1589-1599.

[6] a) S. Bivaud, J. Y. Balandier, M. Chas, M. Allain, S. Goeb, M. Salle, J. Am. Chem. Soc. 2012, 134, 11968-11970; b) S. Bivaud, S. Goeb V. Croue, P. I. Dron, M. Allain, M. Salle, J. Am. Chem. Soc. 2013 $135,10018-10021$

[7] a) D. Canevet, M. Salle, G. X. Zhang, D. Q. Zhang, D. B. Zhu, Chem Commun. 2009, 2245-2269; b) J. L. Segura, N. Martin, Angew. Chem. Int. Ed. 2001, 40, 1372-1409.

[8] a) J. O. Jeppesen, J. Becher, Eur. J. Org. Chem. 2003, 3245-3266; b J. O. Jeppesen, K. Takimiya, F. Jensen, T. Brimert, K. Nielsen, N Thorup, J. Becher, J. Org. Chem. 2000, 65, 5794-5805; c) J. Y. Balandier, A. Belyasmine, M. Salle, Synthesis 2006, 2815-2817.

[9] E. J. Corey, Y. Tian, Org. Lett. 2005, 7, 5535-5537.

[10] G. W. T. M. J. Frisch, H. B. Schlegel, G. E. Scuseria, M. A. Rob, J. R. Cheeseman, J. A. Montgomery Jr., T. Vreven, K. N. Kudin, J. C. Burant, J. M. Millam, S. S. Iyengar, J. Tomasi, V. Barone, B Mennucci, M. Cossi, G. Scalmani, N. Rega, G. A. Petersson, H Nakatsuji, M. Hada, M. Ehara, K. Toyota, R. Fukuda, J. Hasegawa, M. Ishida, T. Nakajima, Y. Honda, O. Kitao, H. Nakai, M. Klene, X Li, J. E. Knox, H. P. Hratchian, J. B. Cross, V. Bakken, C. Adamo, J. Jaramillo, R. Gomperts, R. E. Stratmann, O. Yazyev, A. J. Austin, R. Cammi, C. Pomelli, J. W. Ochterski, P. Y. Ayala, K. Morokuma, G. A. Voth, P. Salvador, J. J. Dannenberg, V. G. Zakrzewski, S. Dapprich, A. D. Daniels, M. C. Strain, O. Farkas, D. K. Malick, A. D. Rabuck, K. Raghavachari, J. B. Foresman, J. V. Ortiz, Q. Cui, A. G. Baboul, S. Clifford, J. Cioslowski, B. B. Stefanov, G. Liu, A Liashenko, P. Piskorz, I. Komaromi, R. L. Martin, D. J. Fox, T. Keith, M. A. Al-Laham, C. Y. Peng, A. Nanayakkara, M Challacombe, P. M. W. Gill, B. Johnson, W. Chen, M. W. Wong, C. Gonzalez, J. A. Pople, Gaussian 03 (Gaussian, Inc., Wallingford, CT, 2003).

[11] a) D. C. Caskey, T. Yamamoto, C. Addicott, R. K. Shoemaker, J. Vacek, A. M. Hawkridge, D. C. Muddiman, G. S. Kottas, J. Michl, P. J. Stang, J. Am. Chem. Soc. 2008, 130, 7620-7628; b) Y. Yamanoi, Y. Sakamoto, T. Kusukawa, M. Fujita, S. Sakamoto, K. Yamaguchi, $J$. Am. Chem. Soc. 2001, 123, 980-981.

[12] a) P. Teo, L. L. Koh, T. S. A. Hor, Inorg. Chem. 2008, 47, 6464 6474; b) M. Schweiger, S. R. Seidel, A. M. Arif, P. J. Stang, Angew. Chem. Int. Ed. 2001, 40, 3467-3469; c) S. Ghosh, D. R. Turner, S. R. Batten, P. S. Mukherjee, Dalton Trans. 2007, 1869-1871.

[13] The CV of ligand $\mathbf{4}$ could not be run under these conditions because of a lack of solubility.

[14] G. N. Li, J. Xiong, Y. Liao, L. Sun, Y. Z. Li, J. L. Zuo, Polyhedron 2011, 30, 2473-2478.

[15] N. Lardies, E. Cerrada, M. Laguno, Polyhedron 2006, 25, 2785-2790. 
[16] a) L. K. Keniley, L. Ray, K. Kovnir, L. A. Dellinger, J. M. Hoyt, M. Shatruk, Inorg. Chem. 2010, 49, 1307-1309; b) F. Pop, D. G. Branzea, T. Cauchy, N. Avarvari, Comptes Rendus Chimie 2012, 15, 904-910.

[17] a) R. Kato, Chem. Rev. 2004, 104, 5319-5346; b) A. Zarkadoulas, E. Koutsouri, C. Mitsopoulou, Coord. Chem. Rev. 2012, 256, 2424 2434.

Received: ((will be filled in by the editorial staff)) Published online: ((will be filled in by the editorial staff)) 
Entry for the Table of Contents (Please choose one layout)

\section{Layout 1:}

An expeditive synthesis of electroactive di- and tetratopic ligands based on the bis(pyrrolo)tetrathiafulvalene (BPTTF) framework is described. The coordination-driven self-assembly of these ligands with cis-protected Pt(II) complexes, affords the first examples of $3 \mathrm{D}$ cages incorporating the electronrich TTF unit. These ligands are also able to undergo an original conversion to heteroleptic dithiolene complexes.

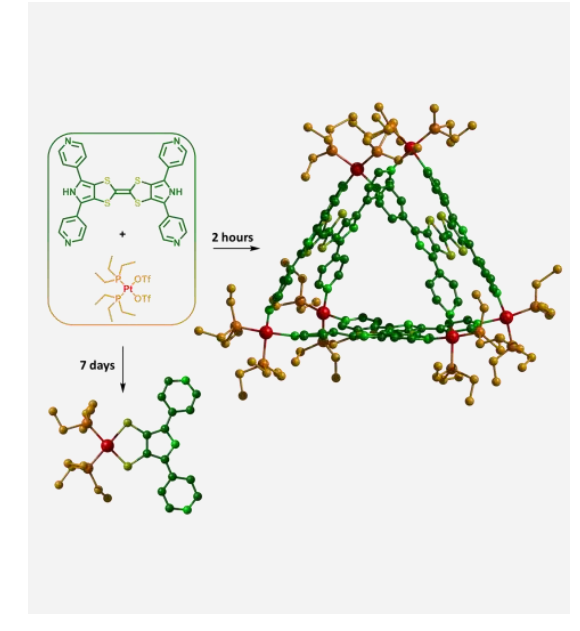

Sébastien Bivaud, Sébastien Goeb, Jean-Yves Balandier, Marcos Chas, Magali Allain and Marc

Sallé*... Page No. -

Page No.

Self-Assembled Cages from the electroactive

Bis(pyrrolo)tetrathiafulvalene (BPTTF) building block

Keywords: Cages / Self-Assembly / Coordination / Tetrathiafulvalene / Redox-active
Text for Table of Contents, Continued max. 350 characters; not the same text as the Abstract. 


\section{Supporting Information}

\section{Self-Assembled Cages from the electroactive Bis(pyrrolo)tetrathiafulvalene (BPTTF) building block $\uparrow$}

Sébastien Bivaud, Sébastien Goeb, Jean-Yves Balandier, Marcos Chas, Magali Allain and Marc Sallé*

Chemicals and instrumentation



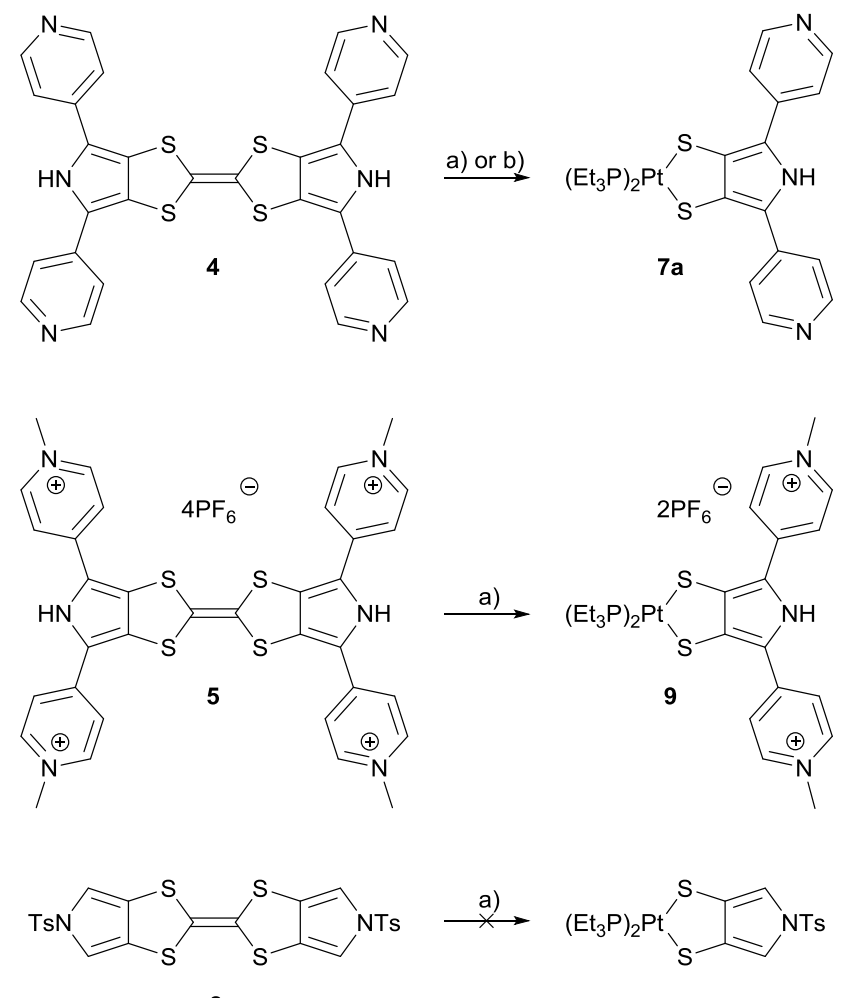

8

a) $\left(\mathrm{Et}_{3} \mathrm{P}\right)_{2} \mathrm{Pt}(\mathrm{OTf})_{2}$ (2 equiv.), DMSO, $75^{\circ} \mathrm{C}, 5-7$ days

b) $\left(\mathrm{Et}_{3} \mathrm{P}\right)_{2} \mathrm{Pt}(\mathrm{OTf})_{2}$ (2 equiv.), $\mathrm{AuBr}_{2}$ (TBA) (1 equiv.), DMSO, r.t., $8 \mathrm{~h}$

Scheme S1. Reaction of BPTTF derivatives 4, 5, 8 with 2 equiv. of $\left(\mathrm{Et}_{3} \mathrm{P}\right)_{2} \mathrm{Pt}(\mathrm{OTf})_{2}$.

\section{Chemicals and instrumentation}

\section{Chemicals}

All reagents were of commercial reagent grade and were used without further purification. Compound BPTTF ${ }^{1}$ MPTTF ${ }^{2} \quad$ complexes $\quad c i s-\mathrm{Pt}\left(\mathrm{PEt}_{3}\right)_{2}(\mathrm{OTf})_{2}{ }^{3}{ }^{3}$ and $\quad c i s-\mathrm{Pt}(\mathrm{dppp})(\mathrm{OTf})_{2}{ }^{4} \quad(\mathrm{dppp}=1,3-$ bis(diphenylphosphino)propane; OTf = trifluoromethane-sulfonate) were synthesized as described in literature. Silica gel chromatography was performed with a SIGMA Aldrich Chemistry $\mathrm{SiO}_{2}$ (pore size $60 \AA, 40-63 \mu \mathrm{m}$ technical grades).

\section{Instrumentation}

The $300.3\left({ }^{1} \mathrm{H}\right), 75.5\left({ }^{13} \mathrm{C}\right), 121.6\left({ }^{31} \mathrm{P}\right)$ and $282.6 \mathrm{MHz}\left({ }^{19} \mathrm{~F}\right)$ NMR spectra were recorded at room temperature using perdeuterated solvents as internal standards $\left({ }^{1} \mathrm{H}\right)$, external $\mathrm{H}_{3} \mathrm{PO}_{4}$ solution $\left({ }^{31} \mathrm{P}\right)$ and $\mathrm{CFCl}_{3}\left({ }^{19} \mathrm{~F}\right)$, on a NMR Bruker Avance III 300 spectrometer. MALDI-TOF-MS spectra were recorded on a MALDI-TOF Bruker Bifle III instrument using a positive-ion mode. ESI-MS spectra were achieved on a Bruker MicrO-Tof-Q 2 spectrometer in $\mathrm{CH}_{3} \mathrm{CN}$ (100 mmol). Cyclic voltammetry experiments were carried out on an ALS electrochemical analyzer model 660, and the conditions were the following: $0.1 \mathrm{M}$ $\mathrm{nBu}_{4} \mathrm{NPF}_{6}$ in distilled acetonitrile, $\mathrm{Ag} / \mathrm{Ag}+$ reference electrode, $\mathrm{GC}$ or Pt working electrode, and $\mathrm{Pt}$ counter electrode, $50 \mathrm{mV} . \mathrm{s}^{-1}$, calibrated using internal ferrocen. Elemental analyses were achieved on a 
Thermo Electron analyser. X-ray diffraction data for the 5 single-crystals were collected at different temperatures on a BRUKER KappaCCD diffractometer (for $\mathbf{2 . H 2 O}, \mathbf{5}, \mathbf{6 a}, \mathbf{7 b}$ ), and on a STOE-IPDS diffractometer (for 2), both equipped with a graphite monochromator utilizing MoK $\alpha$ radiation $(\lambda=$ $0.71073 \AA$ ). The structures were solved by direct (SIR92 (Altomare et al.,1993) or SHELXS) and refined on $\mathrm{F}^{2}$ by full matrix least-squares techniques using SHELX97 (G.M. Sheldrick, 1998) package. All non $\mathrm{H}$ atoms were refined anisotropically, except for compound $\mathbf{6 a}$ for which $\mathrm{C}, \mathrm{O}$ and $\mathrm{N} 5$ atoms were refined isotropically. Absorption was corrected by SADABS program (Sheldrick, Bruker, 2008) (for 5,

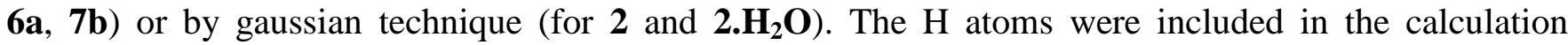
without refinement (for 2, 5, 6a, $7 \mathbf{b}$ (36 H on $39 \mathrm{H}$ atoms)) or were found by Fourier difference map (for 2. $\mathbf{H}_{2} \mathrm{O}$ and the $3 \mathrm{H}$ atoms on the $\mathrm{N}$ atoms of $\mathbf{7 b}$ ).

\section{Theoritical calculations}

The electronics properties of BPTTF, MPTTF, 2 and $\mathbf{4}$ were achieved by the Density Functional Theory (DFT) calculations at the Becke3LYP (B3LYP) 6-31G(d,p) level with Gaussian 03 (2009 version).

\section{Experimental procedure and Characterization data}

2-(4,5-bis(methylthio)-1,3-dithiol-2-ylidene)-4,6-bis(1-((trifluoromethyl)sulfonyl)-1,4dihydropyridin-4-yl)-5H-[1,3]dithiolo[4,5-c]pyrrole (1)

To a solution of pyridine $(1.2 \mathrm{~g}, 15.1 \mathrm{mmol})$ in anhydrous dichloromethane $(200 \mathrm{~mL})$ at $-30^{\circ} \mathrm{C}$, was added trifluoromethanesulfonic anhydride $(1.7 \mathrm{~g}, 6.02 \mathrm{mmol})$. After 10 minutes, a white precipitate appeared and a solution of MPTTF $(370 \mathrm{mg}, 1.10 \mathrm{mmol})$ in anhydrous dichloromethane $(50 \mathrm{~mL})$ was added dropwise. The mixture was stirred for $10 \mathrm{~min}$. The reaction was quenched with brine and warmed to room temperature. The organic layer was washed twice with water, dried over magnesium sulfate and concentrated. The residue was purified by chromatography on silica gel, eluting with dichloromethane/cyclohexane (v/v 6/4) to give compound 1 (700 mg, $0.92 \mathrm{mmol}, 84 \%$ ) as an orange solid.

${ }^{1} \mathrm{H}$ NMR $\left(\left(\mathrm{CD}_{3}\right)_{2} \mathrm{SO}\right) \delta 11.16(\mathrm{~s}, 1 \mathrm{H}), 6.72\left(\mathrm{~d},{ }^{3} J=8.34 \mathrm{~Hz}, 4 \mathrm{H}\right), 5.27\left(\mathrm{dd},{ }^{3} J=8.34 \&^{3} J=3.97 \mathrm{~Hz}, 4 \mathrm{H}\right)$, $4.21(\mathrm{~s}, 2 \mathrm{H}), 2.42(\mathrm{~s}, 6 \mathrm{H}) ;{ }^{13} \mathrm{C} \mathrm{NMR}\left(\left(\mathrm{CD}_{3}\right)_{2} \mathrm{SO}\right) \delta 125.94,124.07,121.28,119.15\left(\mathrm{q},{ }^{1} J=373.72 \mathrm{~Hz}\right)$, 115.27, 110.34, 107.37, 29.71, 18.42; ${ }^{19} \mathrm{~F}$ NMR ( $\left.\left(\mathrm{CD}_{3}\right)_{2} \mathrm{SO}\right) \delta$-76.84; ESI-MS m/z (calc.): 756,8889; (theo.): 756.8909; IR (KBr): 3365, 1682, 1636, 1404, 1292, 1231, 1200, 1157, 1072, $949 \mathrm{~cm}^{-1} ; \mathrm{mp}=137$ ${ }^{\circ} \mathrm{C}$

2-(4,5-bis(methylthio)-1,3-dithiol-2-ylidene)-4,6-di(pyridin-4-yl)-5H-[1,3]dithiolo [4,5-c]pyrrole (2) To a solution of the triflate derivative $1(700 \mathrm{mg}, 0.92 \mathrm{mmol})$ in methanol $(50 \mathrm{~mL})$, was added under argon potassium tert-butoxide $(1.05 \mathrm{~g}, 9.36 \mathrm{mmol})$ in one portion. The solution was stirred at room temperature for $18 \mathrm{~h}$. Then, the solvent was evaporated until a third and water was added. The resulting 
solid was centrifuged and washed with water and finally dried under vacuum to give compound 2 (350 $\mathrm{mg}, 0.72 \mathrm{mmol}, 81 \%$ ) as an orange powder.

Orange crystals (needles) were obtained by slow diffusion of diethyl ether in tetrahydrofuran.

${ }^{1} \mathrm{H}$ NMR $\left(\left(\mathrm{CD}_{3}\right)_{2} \mathrm{SO}\right) \delta 12.01(\mathrm{brs}, 1 \mathrm{H}), 8.59\left(\mathrm{~d},{ }^{3} \mathrm{~J}=6.07 \mathrm{~Hz}, 4 \mathrm{H}\right), 7.47\left(\mathrm{~d},{ }^{3} J=6.07 \mathrm{~Hz}, 4 \mathrm{H}\right), 2.44(\mathrm{~s}$, $6 \mathrm{H}) ;{ }^{13} \mathrm{C}$ NMR $\left(\left(\mathrm{CD}_{3}\right)_{2} \mathrm{SO}\right) \delta 150.29,136.47,126.00,123.53,120.48,118.50,117.78,111.17,18.51$; ESI-MS m/z (calc.): 488.9503 ; (theo.): 488.9589; IR (KBr): $1599 \mathrm{~cm}^{-1} ; \mathrm{mp}>260^{\circ} \mathrm{C}$

\section{Bis(2,5-di(4-methylpyridin-1-ium)[3,4-d]pyrrolotetrathiafulvalene (5)}

To a suspension of compound 4 (30 mg, $51 \mu \mathrm{mol})$ in anhydrous dimethylformamide $(3 \mathrm{~mL})$, was added a large excess of iodomethane $(217 \mathrm{mg}, 1.52 \mathrm{mmol})$. The mixture was stirred at $50^{\circ} \mathrm{C}$ for $4 \mathrm{~h}$. After cooling to room temperature, diethyl ether was added. The resulting precipitate was centrifuged, washed with diethyl ether and dichloromethane. Anion exchange was performed by adding a concentrated solution of potassium hexafluorophosphate in water. The resulting precipitate was centrifuged, washed with water, methanol and diethyl ether to give compound 5 (50 mg, $41 \mu \mathrm{mol}, 82 \%)$ as a dark red powder.

Dark crystals (needles) were obtained by slow diffusion of diethyl ether in dimethylformamide.

${ }^{1} \mathrm{H}$ NMR $\left(\mathrm{CD}_{3} \mathrm{CN}\right) \delta 10.95(\mathrm{brs}, 2 \mathrm{H}, \mathrm{NH}), 8.52\left(\mathrm{~d},{ }^{3} J=6.0 \mathrm{~Hz}, 8 \mathrm{H}\right), 7.97\left(\mathrm{~d},{ }^{3} J=6.0 \mathrm{~Hz}, 8 \mathrm{H}\right), 4.23(\mathrm{~s}$, $12 \mathrm{H}) ;{ }^{31} \mathrm{P}$ NMR $\left(\mathrm{CD}_{3} \mathrm{CN}\right) \delta-132.12--155.50\left(\mathrm{~m}, 4 \mathrm{P}, \mathrm{PF}_{6}\right) ;{ }^{19} \mathrm{~F} \mathrm{NMR}\left(\mathrm{CD}_{3} \mathrm{CN}\right) \delta-75.09\left(\mathrm{~d},{ }^{2} J_{P-F}=\right.$ $716.1 \mathrm{~Hz}, 24 \mathrm{~F})$; ESI m/z: $\left[\mathrm{C}_{34} \mathrm{H}_{30} \mathrm{~N}_{6} \mathrm{~S}_{4}\right]^{2+}: 324.27$; $\left[\mathrm{C}_{34} \mathrm{H}_{30} \mathrm{~N}_{6} \mathrm{~S}_{4}\right]^{3+}: 216.49$; $\left[\mathrm{C}_{34} \mathrm{H}_{30} \mathrm{~N}_{6} \mathrm{~S}_{4}\right]^{4+}: 162.65$; $\left[\mathrm{PF}_{6}\right]^{-}: 144.83 ; \mathrm{mp}=>260^{\circ} \mathrm{C}$

\section{Cage (6b)}

A mixture of $4(10.0 \mathrm{mg}, 17 \mu \mathrm{mol})$ and Pt(dppp)(OTf) 2 (30.8 mg, $34 \mu \mathrm{mol})$ in DMSO (1 mL) was heated at $75^{\circ} \mathrm{C}$ for $12 \mathrm{~h}$ under argon. Then, ethyl acetate was added and the mixture was centrifuged. The residue was washed with ethyl acetate, diethyl ether and dried under vacuum to give $\mathbf{6 b}$ (31.4 mg, 4.36 $\mu \mathrm{mol}, 77 \%)$. as a dark red solid.

${ }^{1} \mathrm{H}$ NMR $\left(\left(\mathrm{CD}_{3}\right)_{2} \mathrm{SO}\right) \delta 12.11$ (s, 6H), 9.22 (br s, 12H), 8.11-7.18 (m, 144H), 6.52 (br s, 12H); ${ }^{31} \mathrm{P}$ NMR $\left(\left(\mathrm{CD}_{3}\right)_{2} \mathrm{SO}\right) \delta-13.6\left(J_{P t-P}=3324.59 \mathrm{~Hz}\right) ;{ }^{19} \mathrm{~F}$ NMR $\left(\left(\mathrm{CD}_{3}\right)_{2} \mathrm{SO}\right) \delta$-79.94; ESI-MS m/z: $976.78\left(\left[6 \mathbf{6}-3 \mathrm{H}^{+}-\right.\right.$ 9OTf $\left.]^{6+}\right), 1001.77\left(\left[\mathbf{6 b}-2 \mathrm{H}^{+}-8 \mathrm{OTf}\right]^{6+}\right), 1201.92\left(\left[\mathbf{6 b}-3 \mathrm{H}^{+}-8 \mathrm{OTf}\right]^{5+}\right), 1231,92\left(\left[\mathbf{6 b}-2 \mathrm{H}^{+}-7 \mathrm{OTf}\right]^{5+}\right)$; Anal. Calcd for $\left[\mathrm{C}_{174} \mathrm{H}_{234} \mathrm{~F}_{36} \mathrm{~N}_{18} \mathrm{O}_{36} \mathrm{P}_{12} \mathrm{Pt}_{6} \mathrm{~S}_{24}\right]$ : C: 33.98, H: 3.84, N: 4.10, S: 12.51, O: 9.37; Found: C: 33.68, $\mathrm{H}: 3.88, \mathrm{~N}: 3.10, \mathrm{~S}: 13.29 ; \mathrm{mp}=>260^{\circ} \mathrm{C}$ 


\section{Dithiolene complex (7a)}

A mixture of $4(10.0 \mathrm{mg}, 17 \mu \mathrm{mol})$ and cis-Pt(PEt $)_{\mathbf{2}} \mathbf{2}_{\mathbf{2}}(\mathbf{O T f})_{\mathbf{2}}(24.8 \mathrm{mg}, 34 \mu \mathrm{mol})$ in anhydrous DMSO (1 $\mathrm{mL}$ ) was heated for 7 days at $75^{\circ} \mathrm{C}$ under argon. Then, the solvent was evaporated under vacuum. The residue was washed with diethyl ether and dried under vacuum to give complex 7a $(25.0 \mathrm{mg}, 24.2 \mu \mathrm{mol}$, $72 \%)$ as a dark red solid.

${ }^{1} \mathrm{H}$ NMR $\left(\left(\mathrm{CD}_{3}\right)_{2} \mathrm{SO}\right) \delta 12.13(\mathrm{~s}, 1 \mathrm{H}), 8.82\left(\mathrm{~d},{ }^{3} \mathrm{~J}=7.0 \mathrm{~Hz}, 4 \mathrm{H}\right), 8.67\left(\mathrm{~d},{ }^{3} J=7.0 \mathrm{~Hz}, 4 \mathrm{H}\right), 2.16(\mathrm{~m}, 12 \mathrm{H})$, $1.12(\mathrm{~m}, 18 \mathrm{H}) ;{ }^{31} \mathrm{P}$ NMR $\left(\left(\mathrm{CD}_{3}\right)_{2} \mathrm{SO}\right) \delta 7.52\left(J_{P t-P}=2746.33 \mathrm{~Hz}\right)$; ESI-MS m/z: 714.1760 (Th: 714.17602)

\section{Dithiolene complex (7b)}

A mixture of 4 (10.0 mg, $17 \mu \mathrm{mol})$ and Pt(dppf)(OTf) 2 (30.8 mg, $34 \mu \mathrm{mol})$ in anhydrous DMSO (1 mL) was heated for 9 days at $75^{\circ} \mathrm{C}$ under argon. Then, the solvent was evaporated under vacuum. The residue was washed with diethyl ether and dried under vacuum to give complex 7b $(33.9 \mathrm{mg}, 27.9 \mu \mathrm{mol}, 83 \%)$ as a dark red solid.

Monocrystals (orange, small lozenges) were obtained by slow diffusion of ether in dichloromethane (liquid-liquid), and characterized by DRX.

${ }^{1} \mathrm{H}$ NMR $\left(\left(\mathrm{CD}_{3}\right)_{2} \mathrm{SO}\right) \delta 11.93(\mathrm{~s}, 1 \mathrm{H}), 8.66\left(\mathrm{~d},{ }^{3} \mathrm{~J}=7.0 \mathrm{~Hz}, 4 \mathrm{H}\right), 8.32\left(\mathrm{~d},{ }^{3} \mathrm{~J}=7.0 \mathrm{~Hz}, 4 \mathrm{H}\right), 7.69-7.48(\mathrm{~m}$, $20 \mathrm{H}) ;{ }^{31} \mathrm{P}$ NMR $\left(\left(\mathrm{CD}_{3}\right)_{2} \mathrm{SO}\right) \delta-5.52(P t-P J=2671.58 \mathrm{~Hz}) ; \delta-80.05 ;$ ESI-MS m/z: 890.1447 (Th: $890.14472)$ 


\section{NMR Spectra}

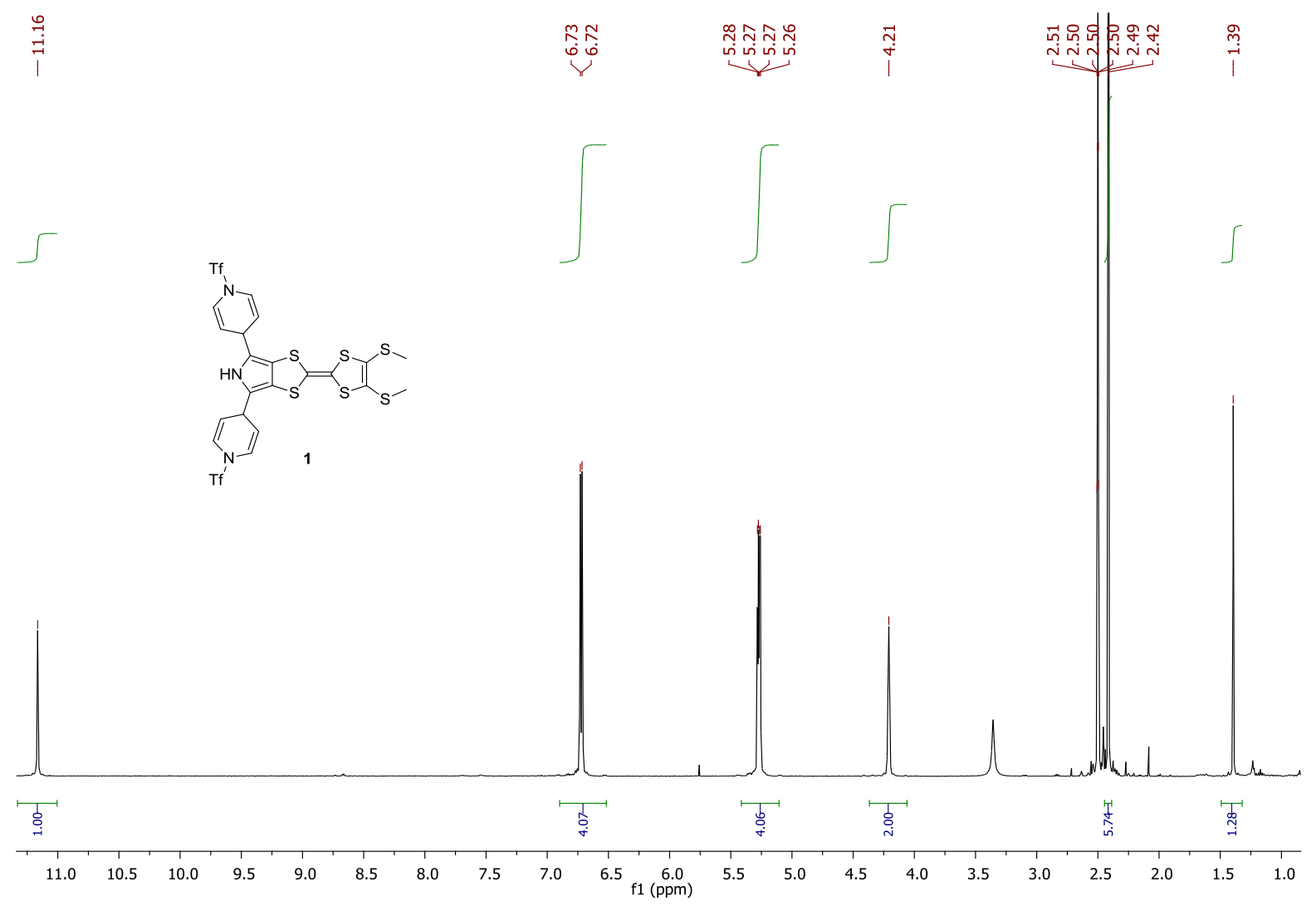

Figure S1. ${ }^{1} \mathrm{H}$ NMR spectrum of $\mathbf{1}$ in DMSO-d 6 

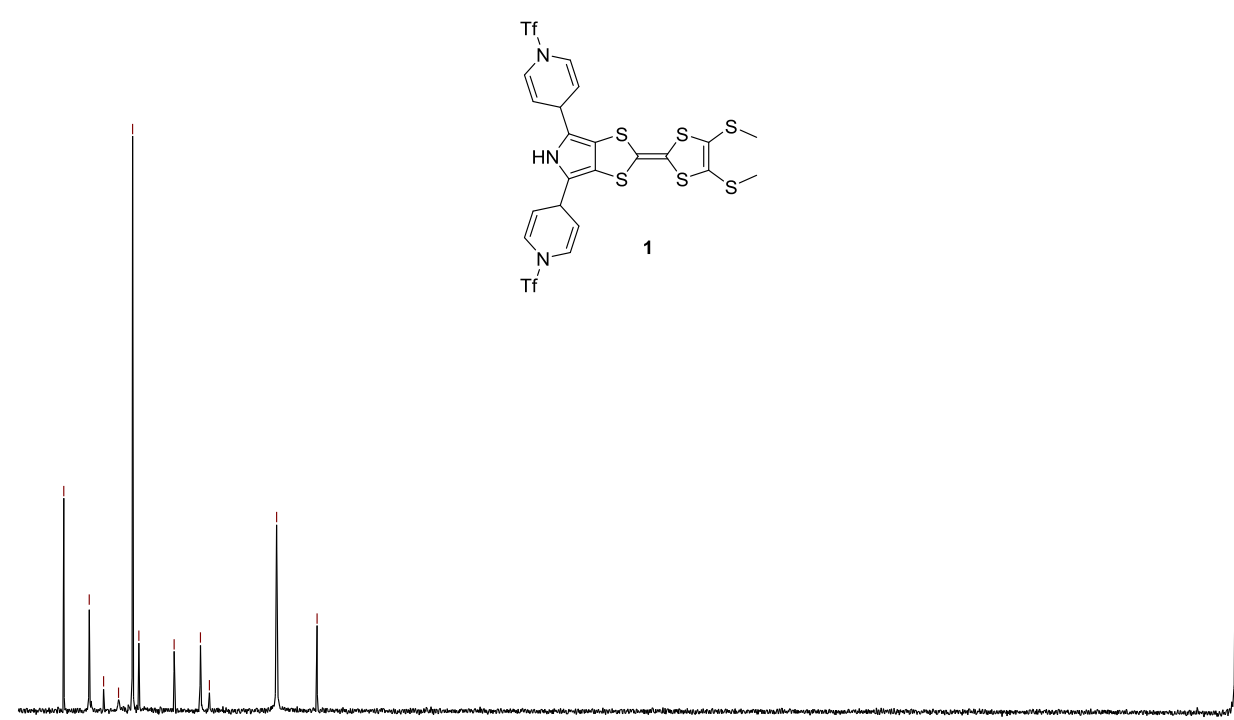

Figure S2. ${ }^{13} \mathrm{C}$ NMR spectrum of $\mathbf{1}$ in DMSO-d 6
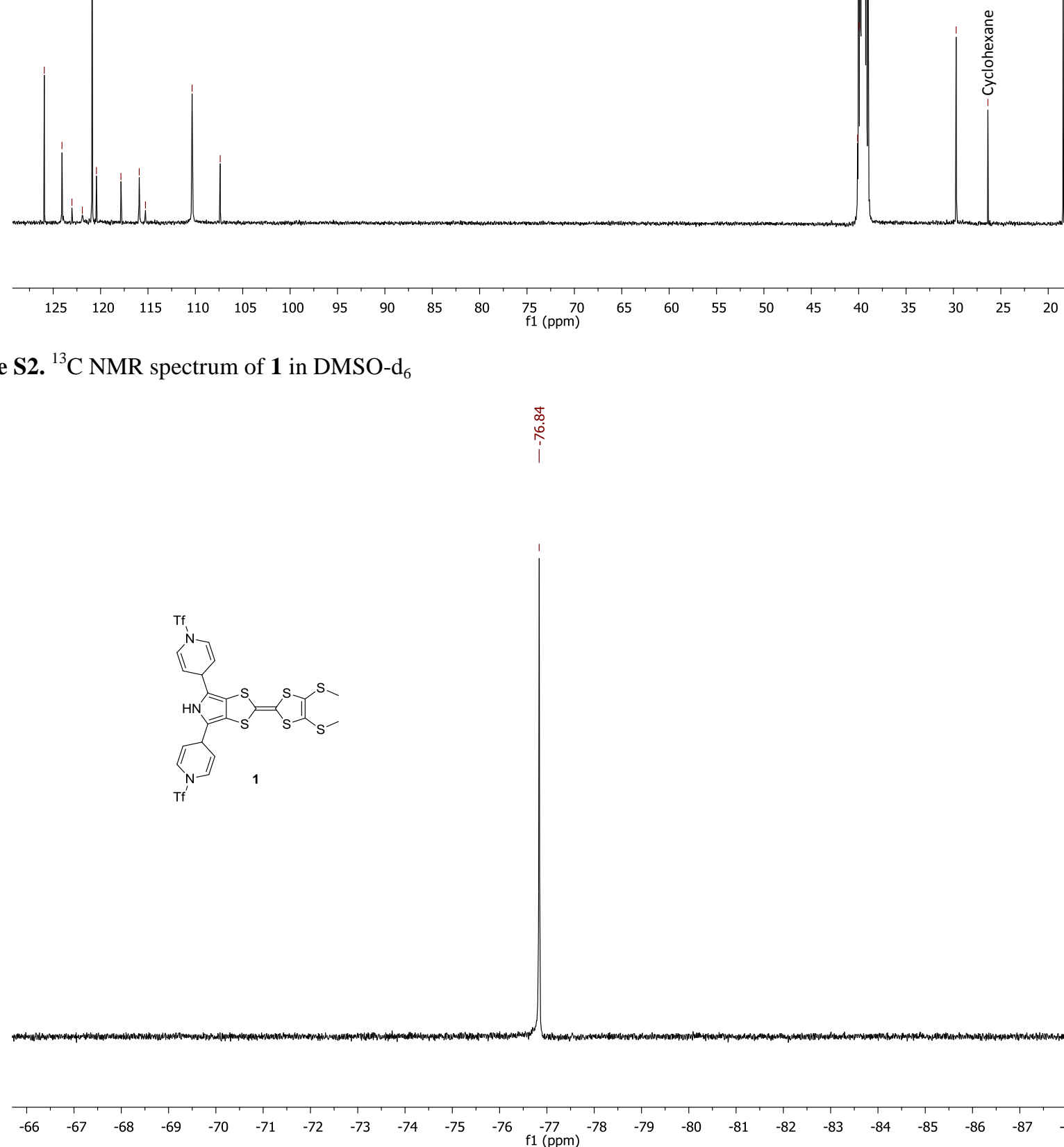

Figure S3. ${ }^{19} \mathrm{~F}$ NMR spectrum of 1 in DMSO- $\mathrm{d}_{6}$ 

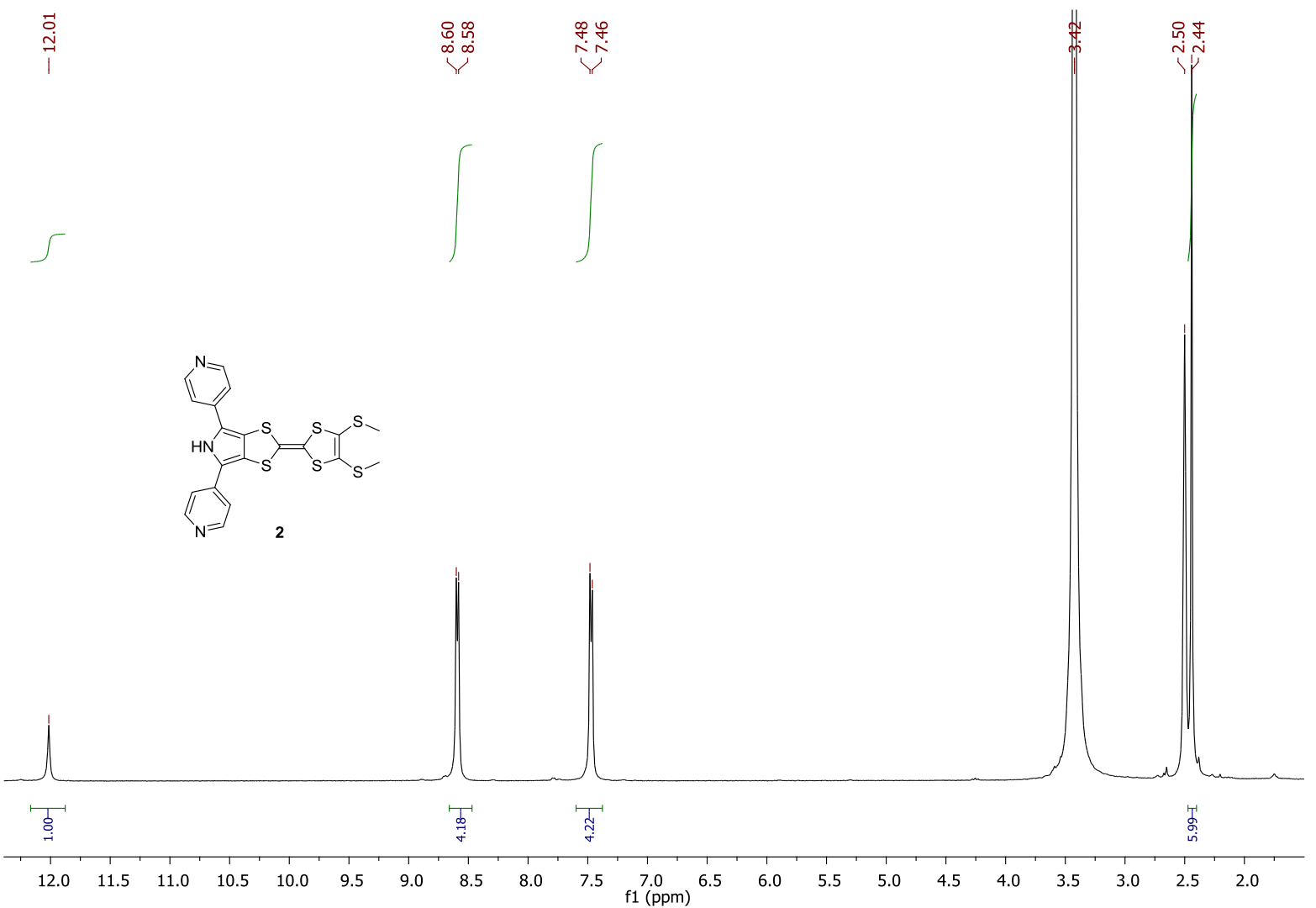

Figure S4. ${ }^{1} \mathrm{H}$ NMR spectrum of 2 in DMSO- $\mathrm{d}_{6}$
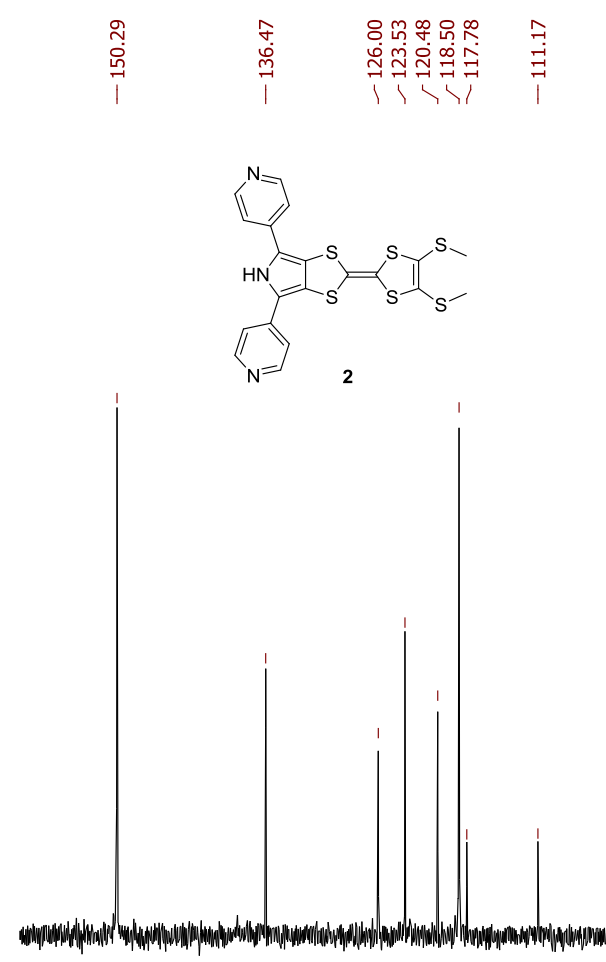

$\begin{array}{llllllllllllllllllllllllllllllllllll}155 & 150 & 145 & 140 & 135 & 130 & 125 & 120 & 115 & 110 & 105 & 100 & 95 & 90 & 85 & 80 & 75 & 70 & 65 & 60 & 55 & 50 & 45 & 40 & 35 & 30 & 25 & 20\end{array}$

Figure S5. ${ }^{13} \mathrm{C}$ NMR spectrum of 2 in DMSO-d ${ }_{6}$ 
$\stackrel{\circ}{\stackrel{8}{-}}$

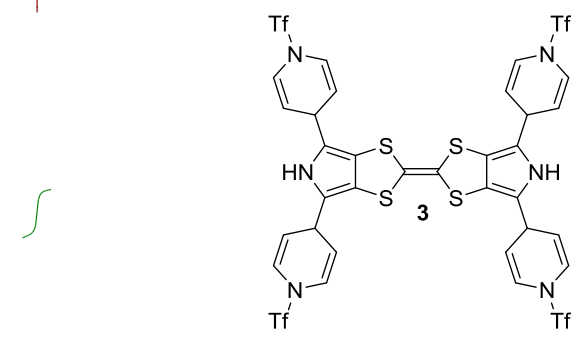

g̊

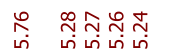

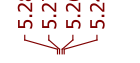

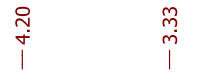

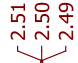
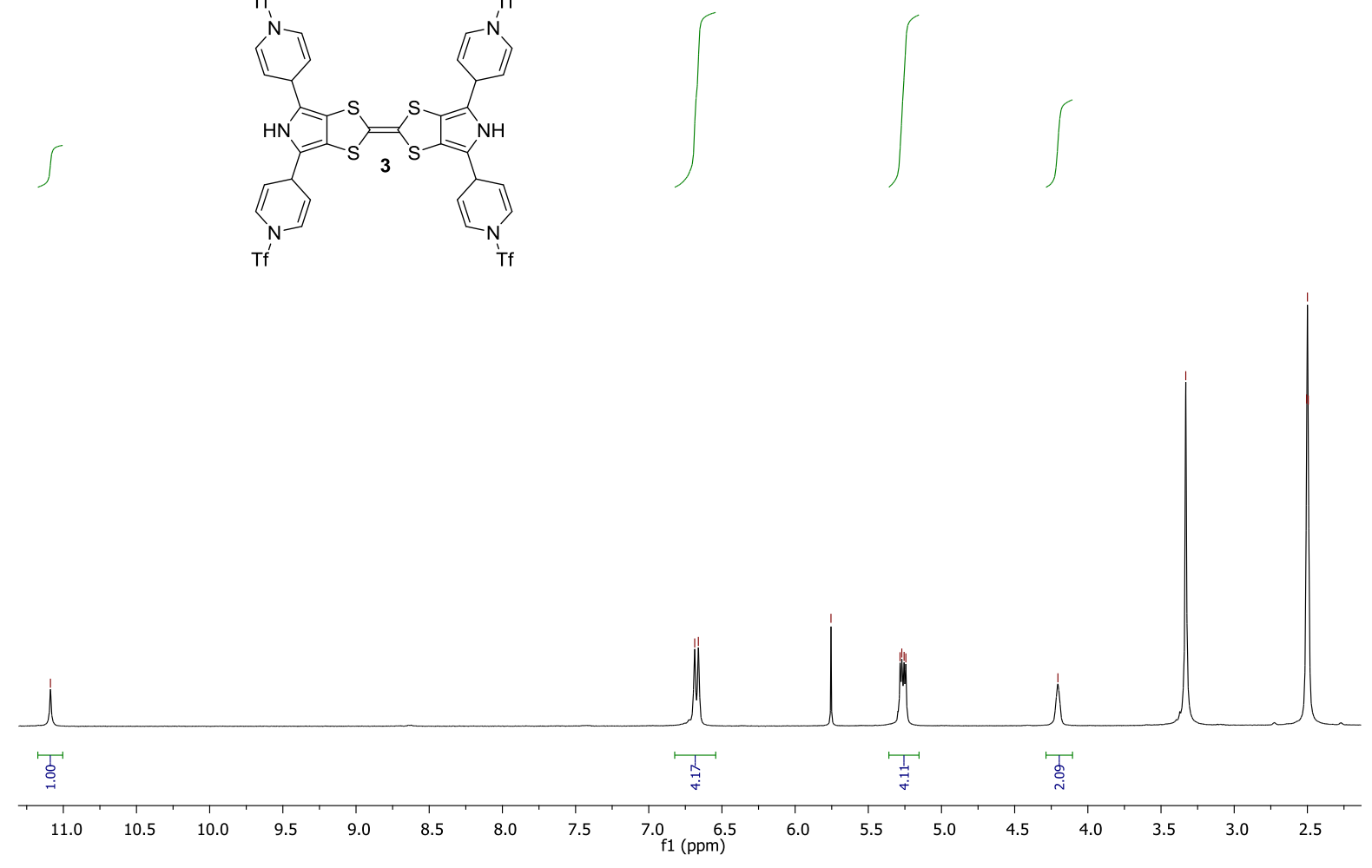

Figure S6. ${ }^{1} \mathrm{H}$ NMR spectrum of $\mathbf{3}$ in DMSO-d
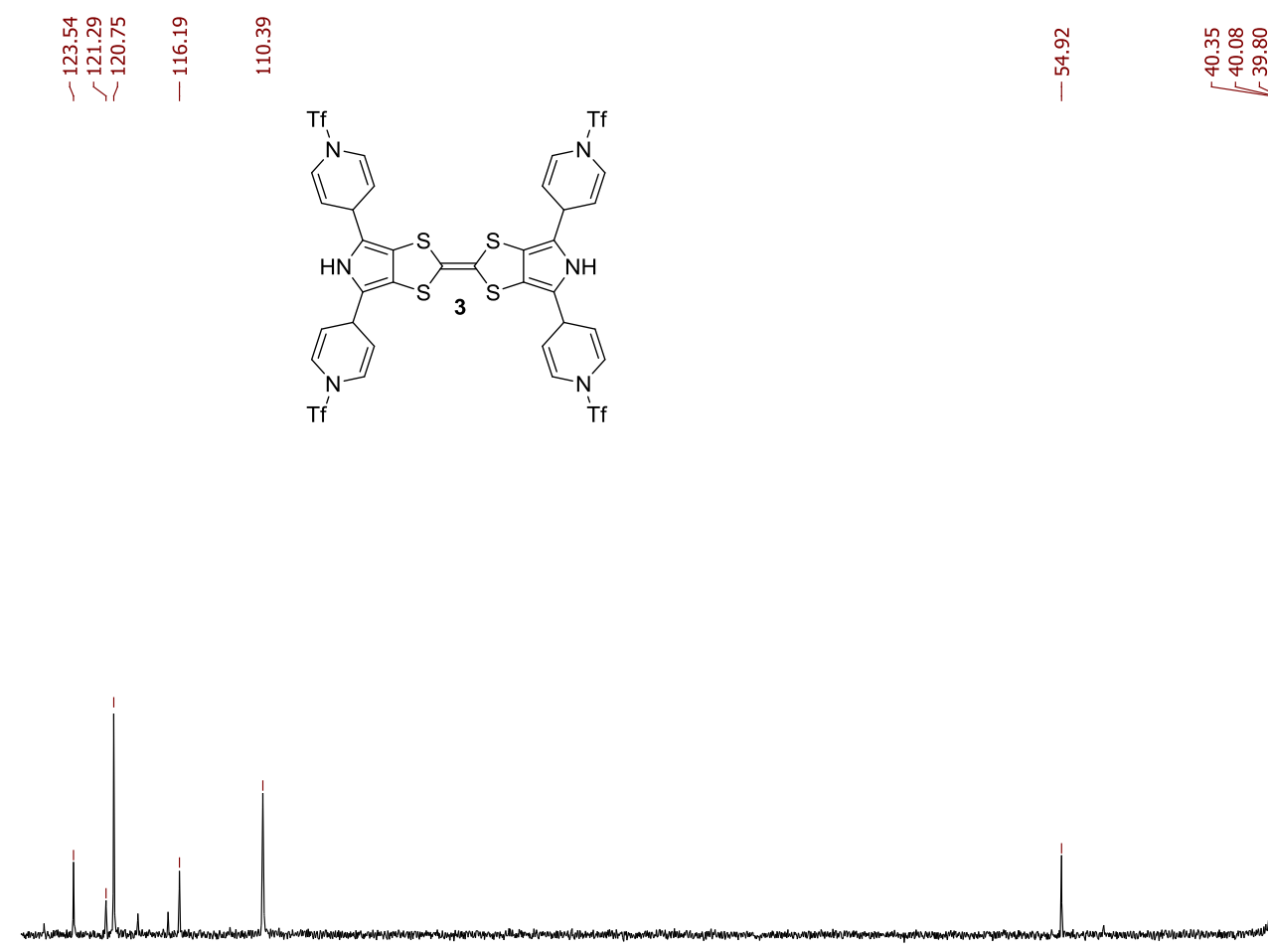

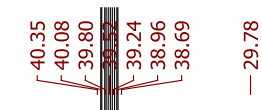

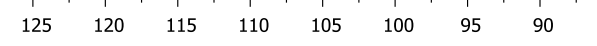

$\begin{array}{lll}85 & 80 & 75 \\ & f 1(\mathrm{ppm})\end{array}$

Figure S7. ${ }^{13} \mathrm{C}$ NMR spectrum of 3 in DMSO- $\mathrm{d}_{6}$ 

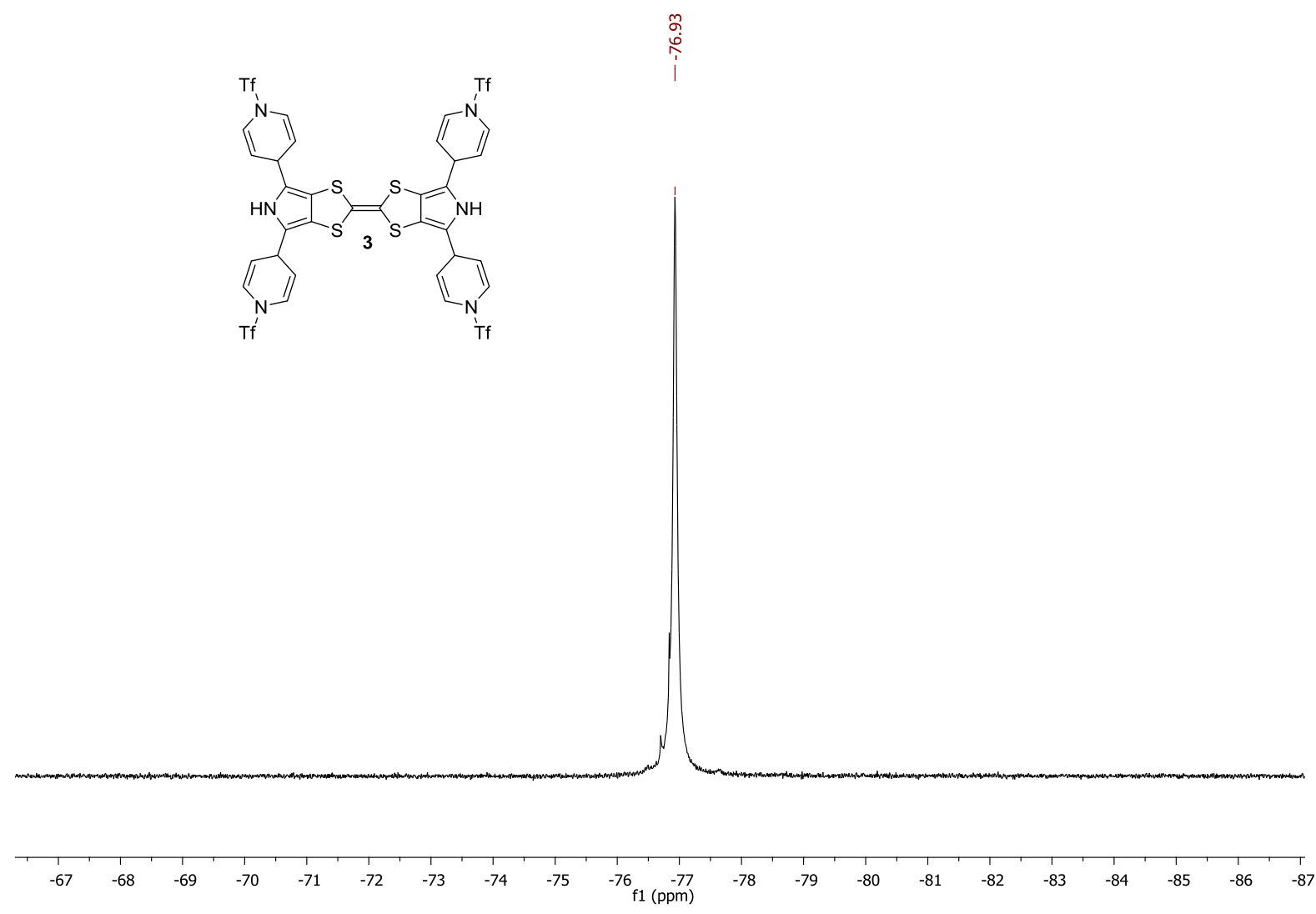

Figure S8. ${ }^{19} \mathrm{~F}$ NMR spectrum of 3 in DMSO-d $\mathrm{d}_{6}$
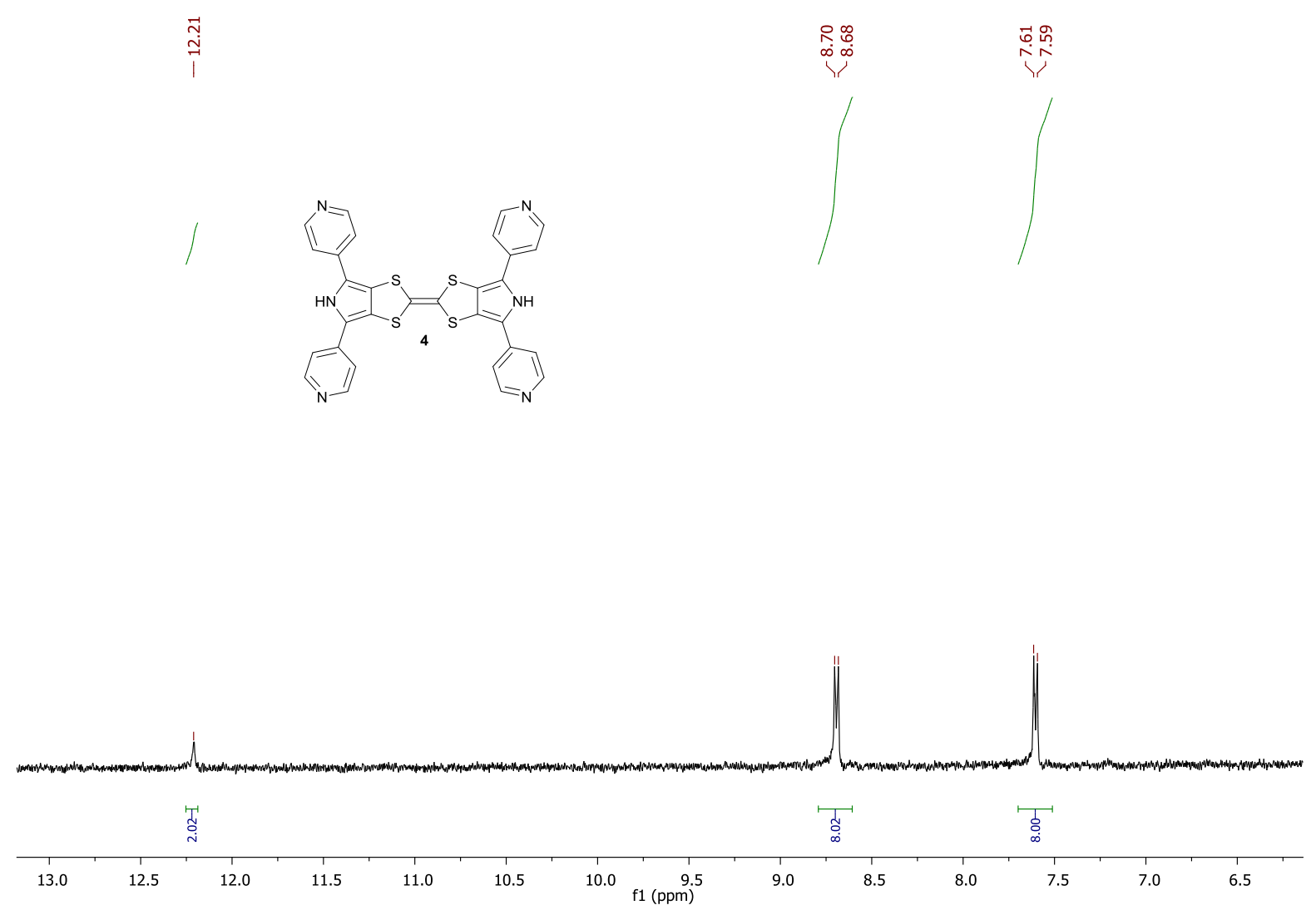

Figure S9. ${ }^{1} \mathrm{H}$ NMR spectrum of $\mathbf{4}$ in DMSO- $\mathrm{d}_{6}$ 

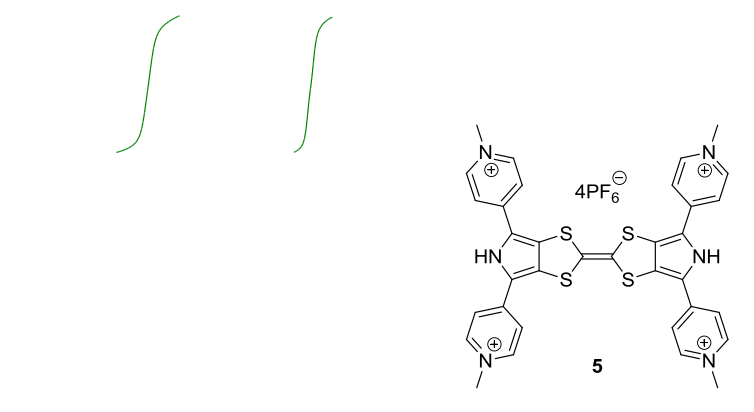

\section{.}

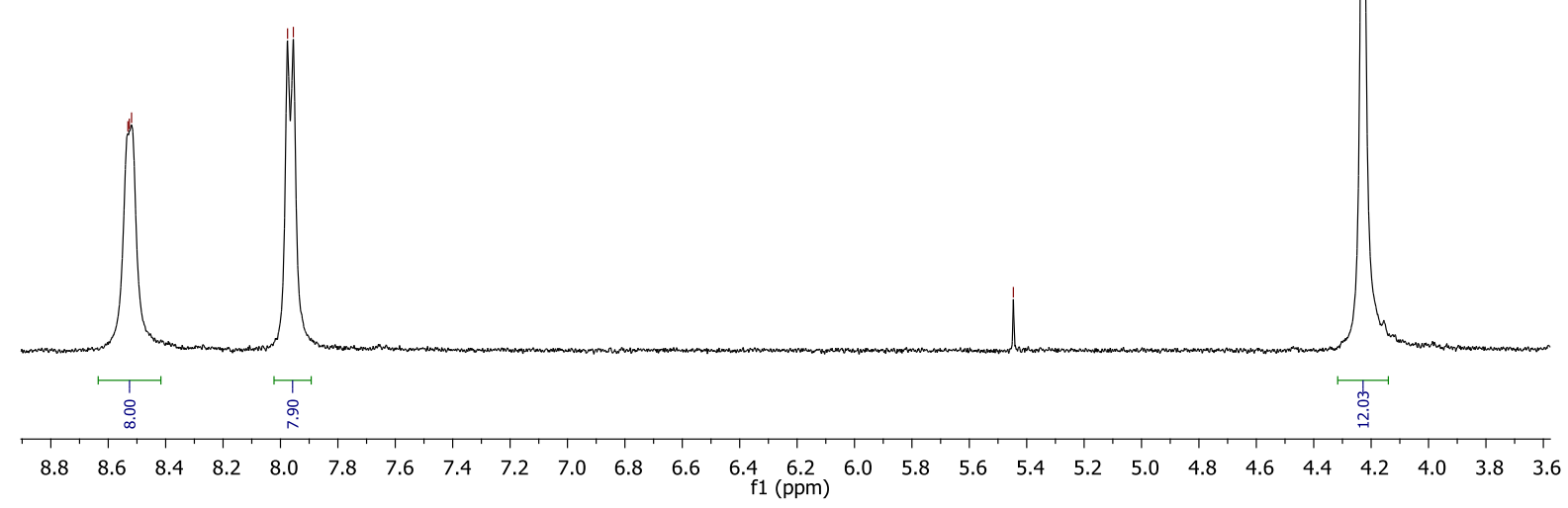

Figure S10. ${ }^{1} \mathrm{H}$ NMR spectrum of 5 in acetonitrile- $\mathrm{d}_{3}$

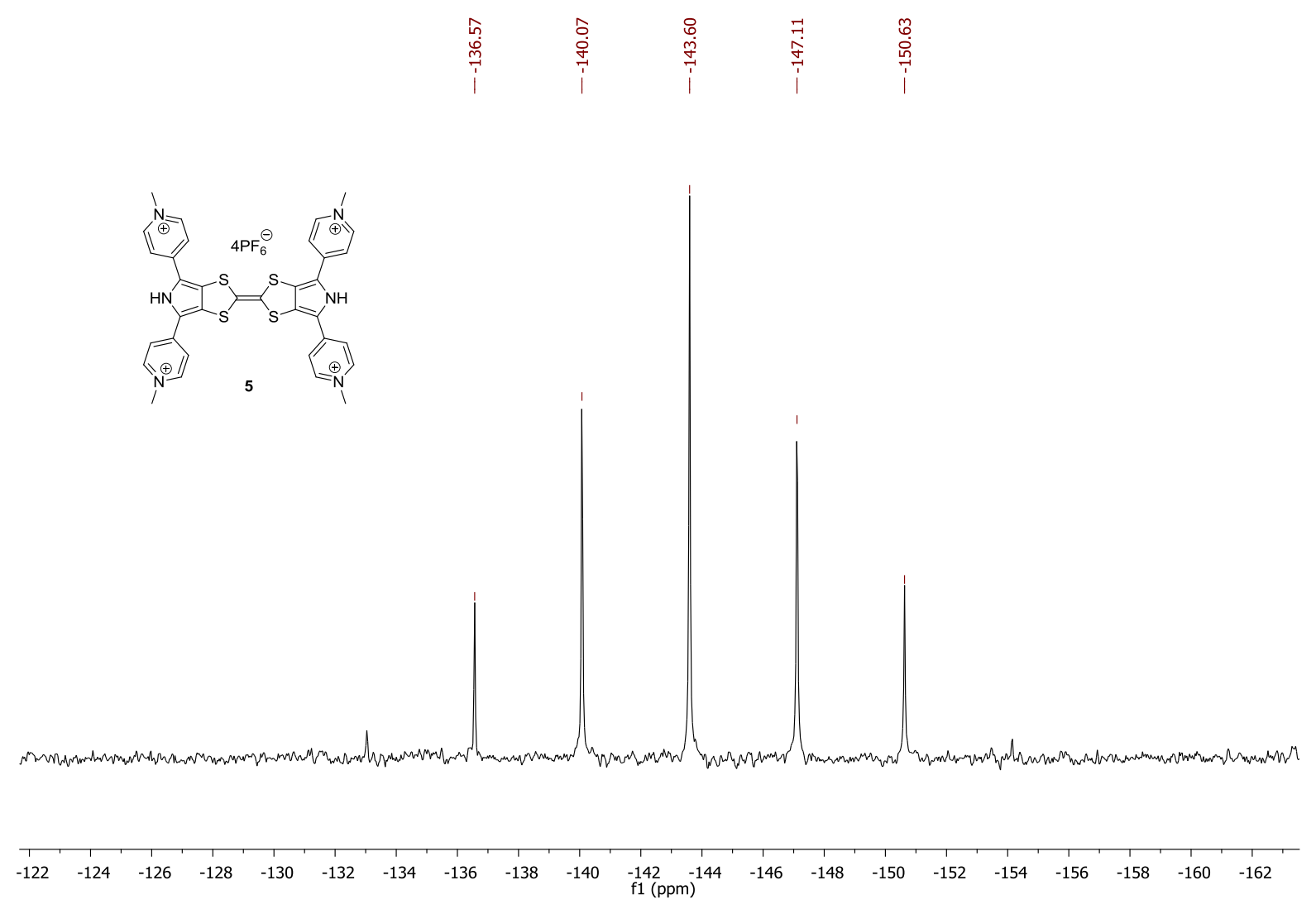

Figure S11. ${ }^{31} \mathrm{P}$ NMR spectrum of $\mathbf{5}$ in acetonitrile- $\mathrm{d}_{3}$ 

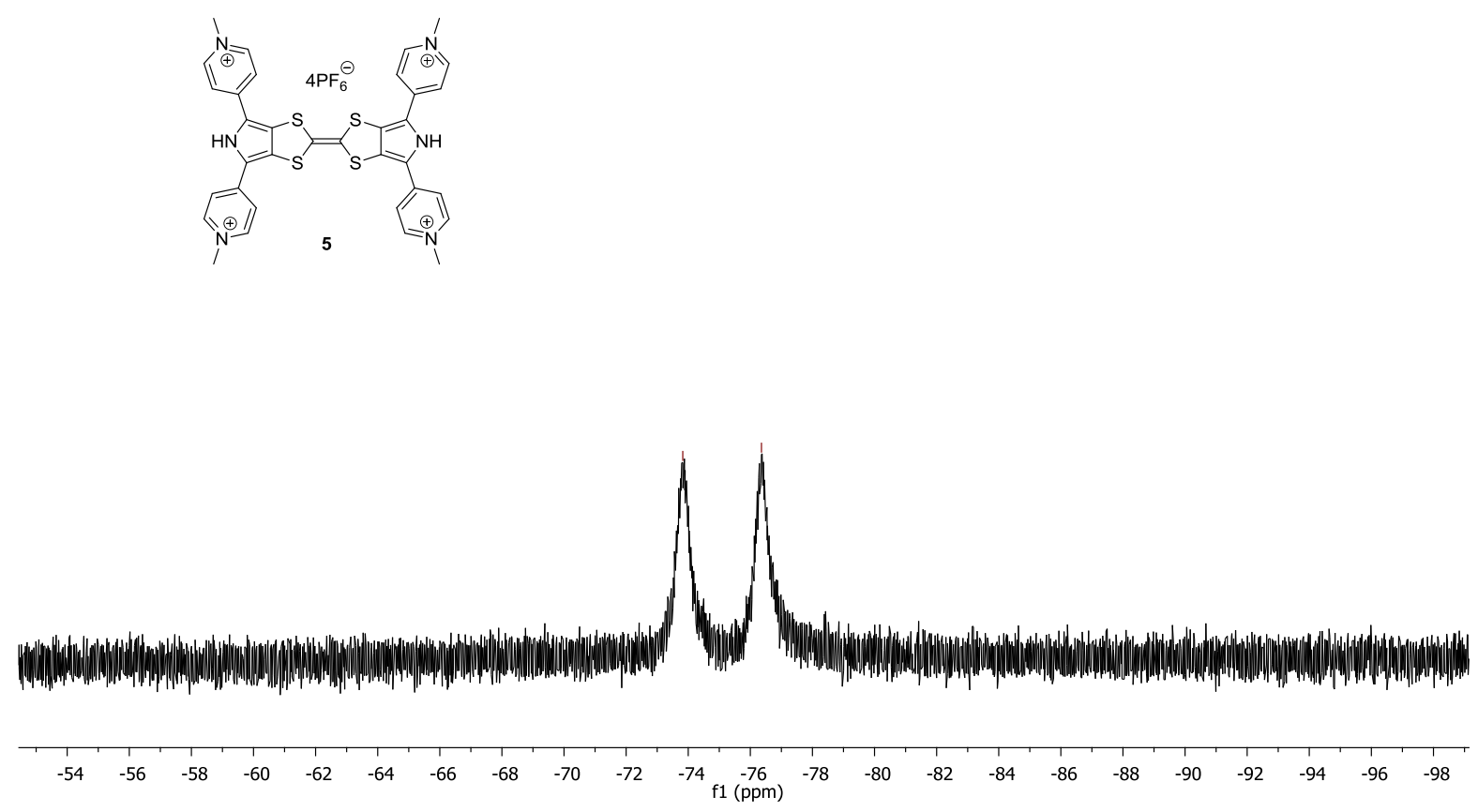

Figure S12. ${ }^{19} \mathrm{~F}$ NMR spectrum of 5 in acetonitrile-d $\mathrm{d}_{3}$

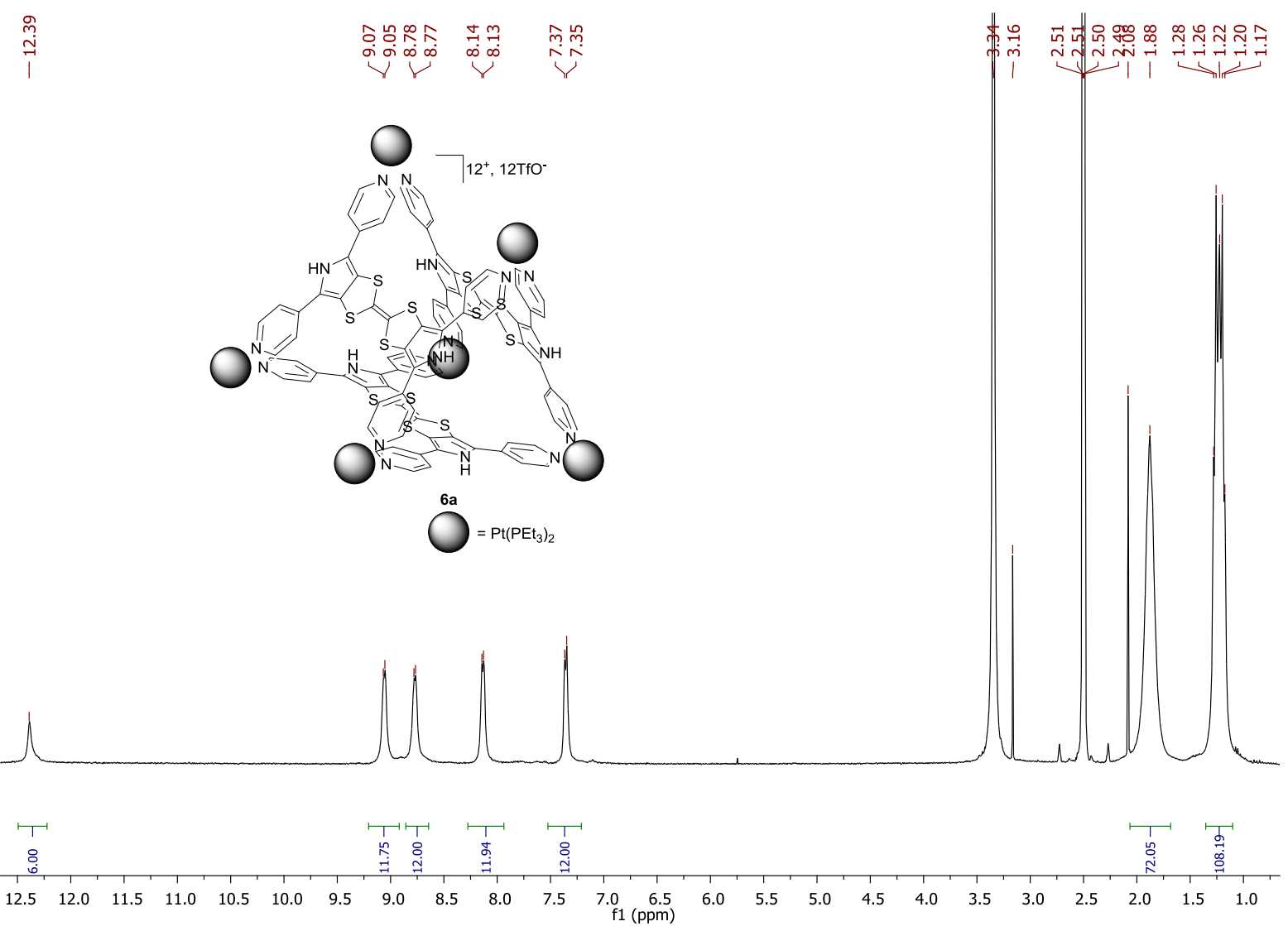

Figure S13. ${ }^{1} \mathrm{H}$ NMR spectrum of 6 a in DMSO-d $\mathrm{d}_{6}$ 


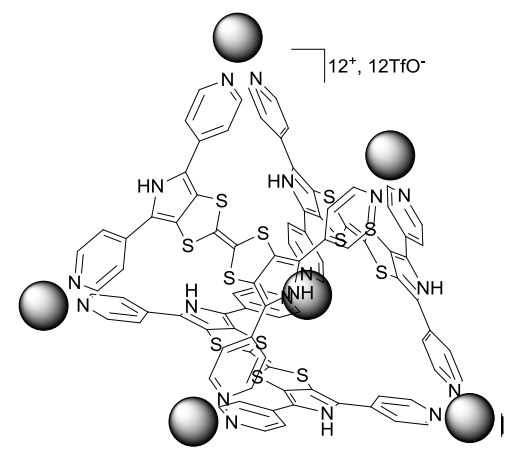

$\bigcirc^{6 \mathrm{a}}=\operatorname{Pt}\left(\mathrm{PEt}_{3}\right)_{2}$

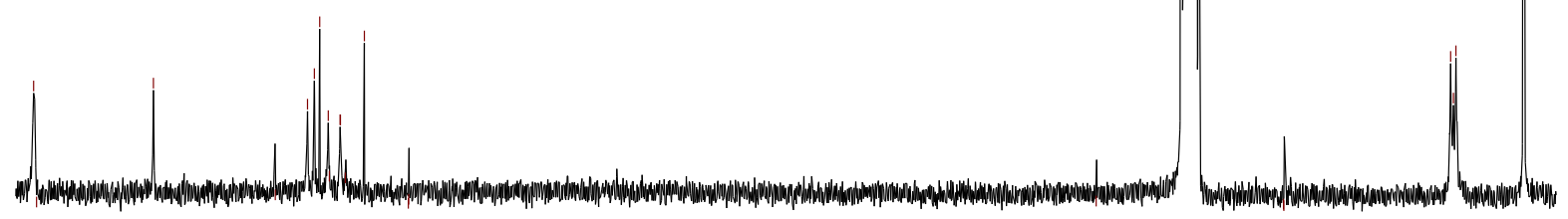

$\begin{array}{llllllllllllllllllllllllllllllllllll}150 & 145 & 140 & 135 & 130 & 125 & 120 & 115 & 110 & 105 & 100 & 95 & 90 & 85 & \begin{array}{c}80 \\ \mathrm{f}(\mathrm{ppm})\end{array} & 70 & 65 & 60 & 55 & 50 & 45 & 40 & 35 & 30 & 25 & 20 & 15 & 10 & 5\end{array}$

Figure S14. ${ }^{13} \mathrm{C}$ NMR spectrum of $6 \mathbf{a}$ in DMSO-d 6

$31 \mathrm{P}$

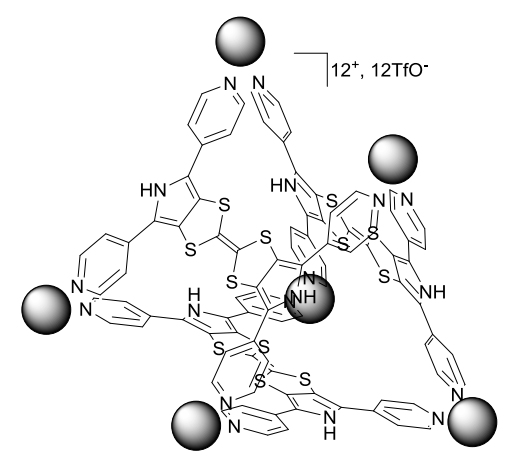

$\bigcap^{6 \mathbf{a}}=\operatorname{Pt}\left(\mathrm{PEt}_{3}\right)_{2}$
$19 F$

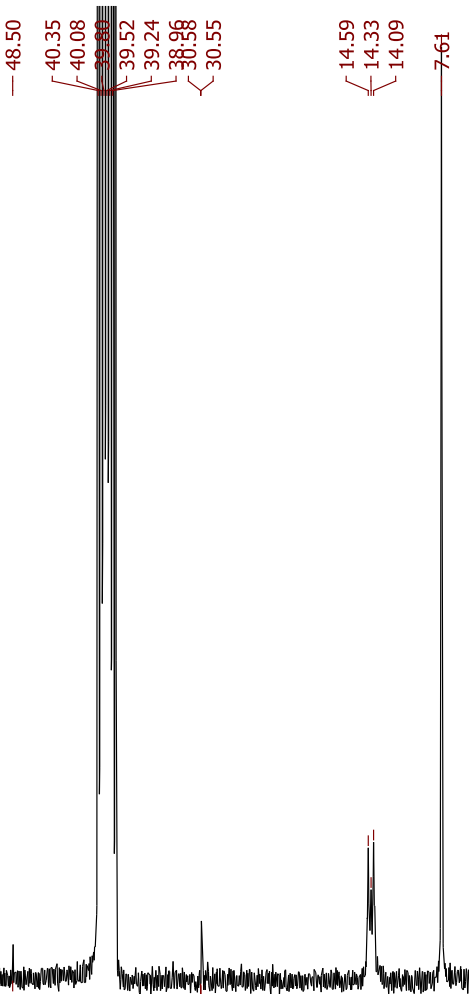

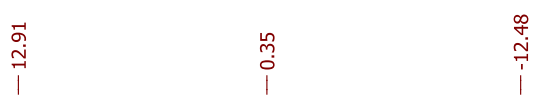

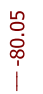

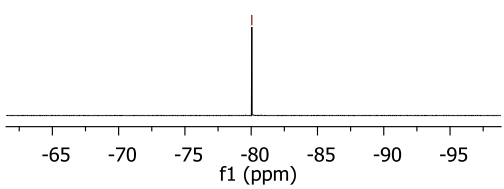

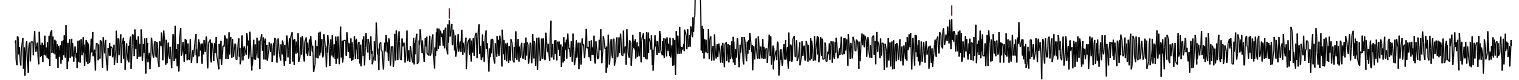

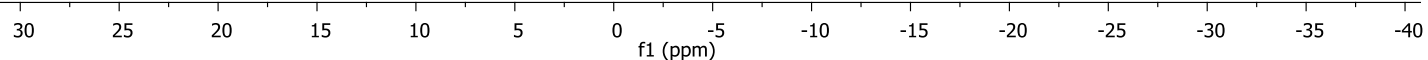

Figure S15. ${ }^{31} \mathrm{P}$ and ${ }^{19} \mathrm{~F}$ NMR spectra of $6 \mathbf{a}$ in DMSO- $\mathrm{d}_{6}$ 


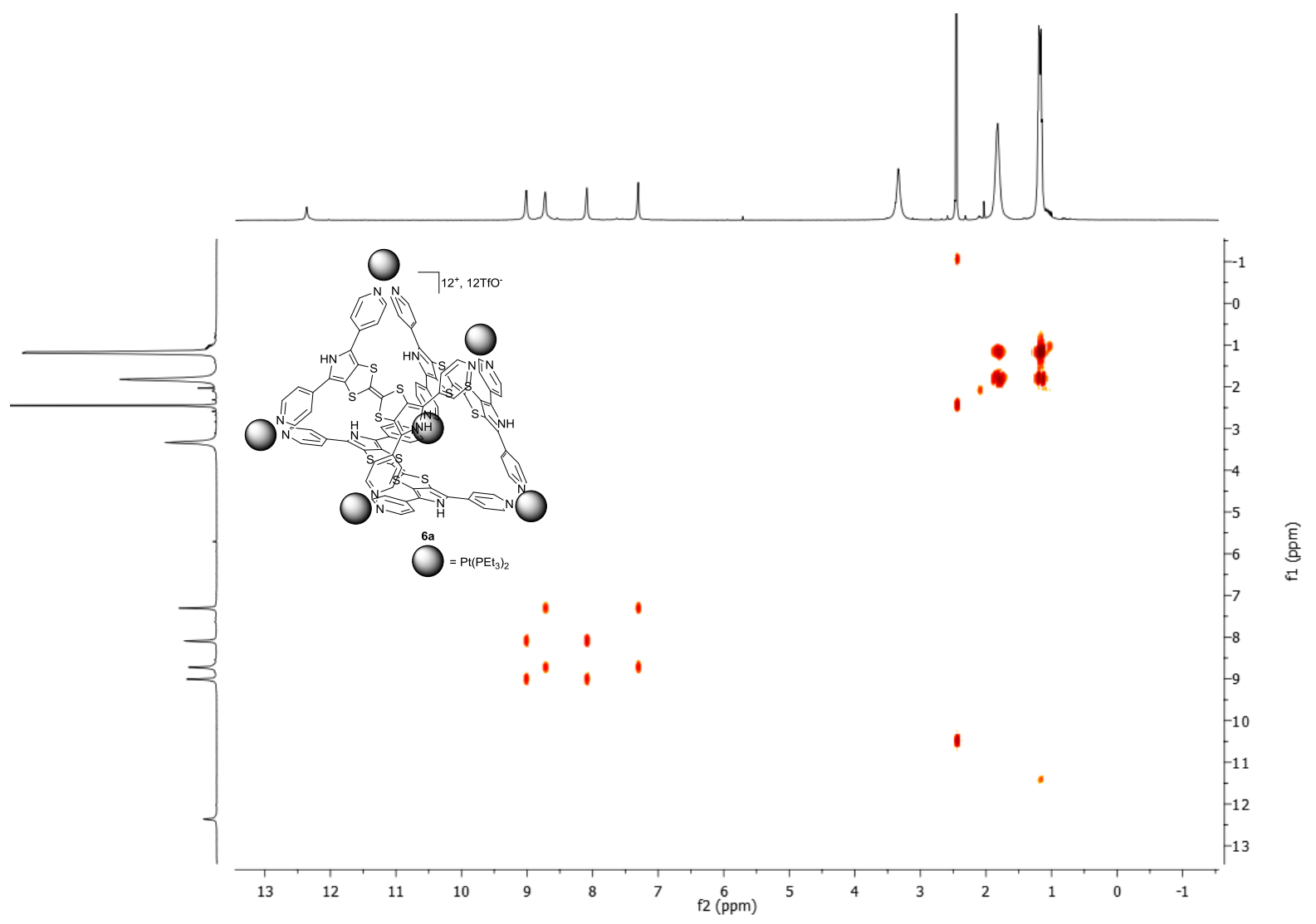

Figure S16. COSY NMR spectra of $\mathbf{6}$ a in DMSO-d $\mathrm{d}_{6}$

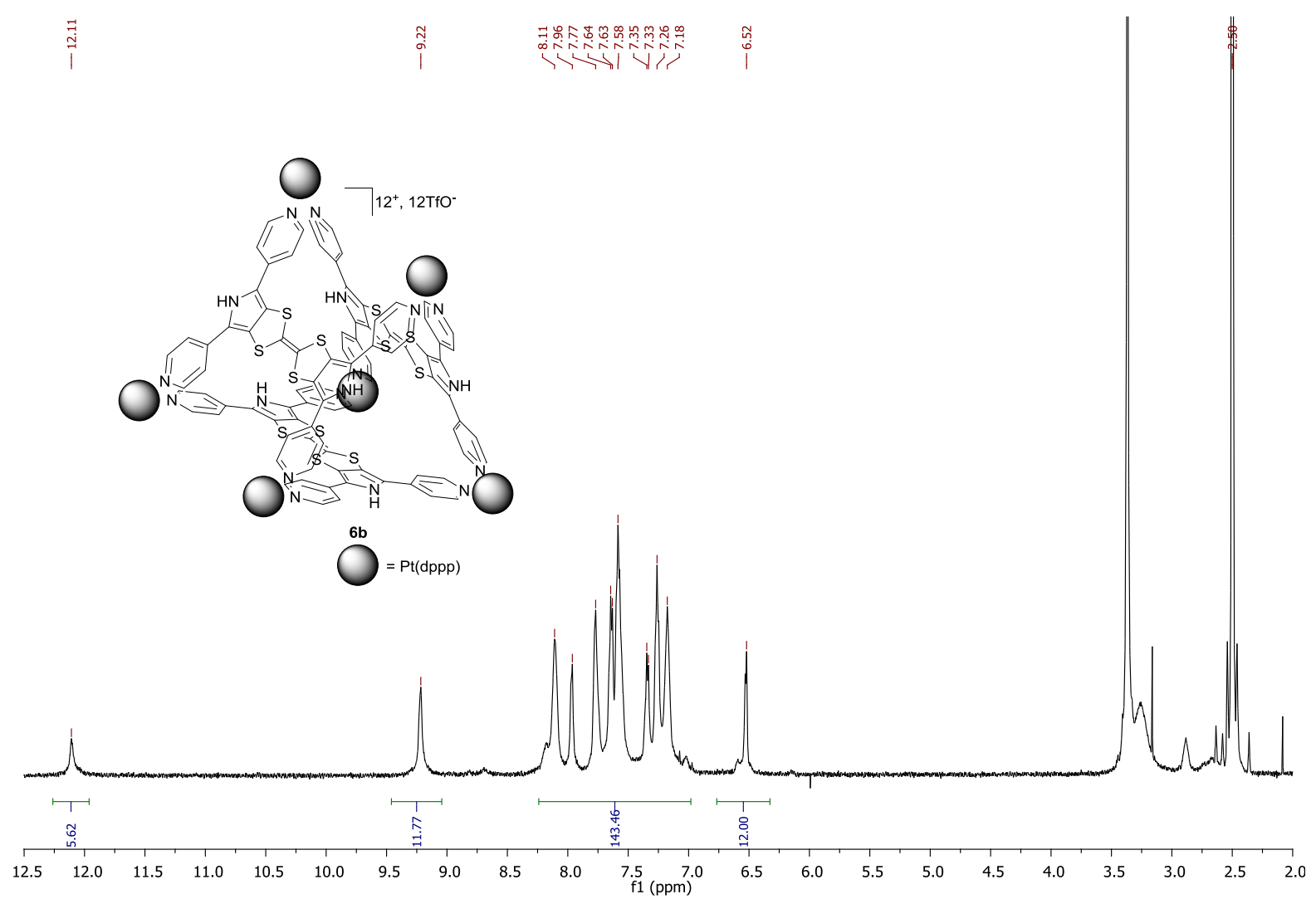

Figure S167. ${ }^{1} \mathrm{H}$ NMR spectrum of $6 \mathbf{b}$ in DMSO- $\mathrm{d}_{6}$ 

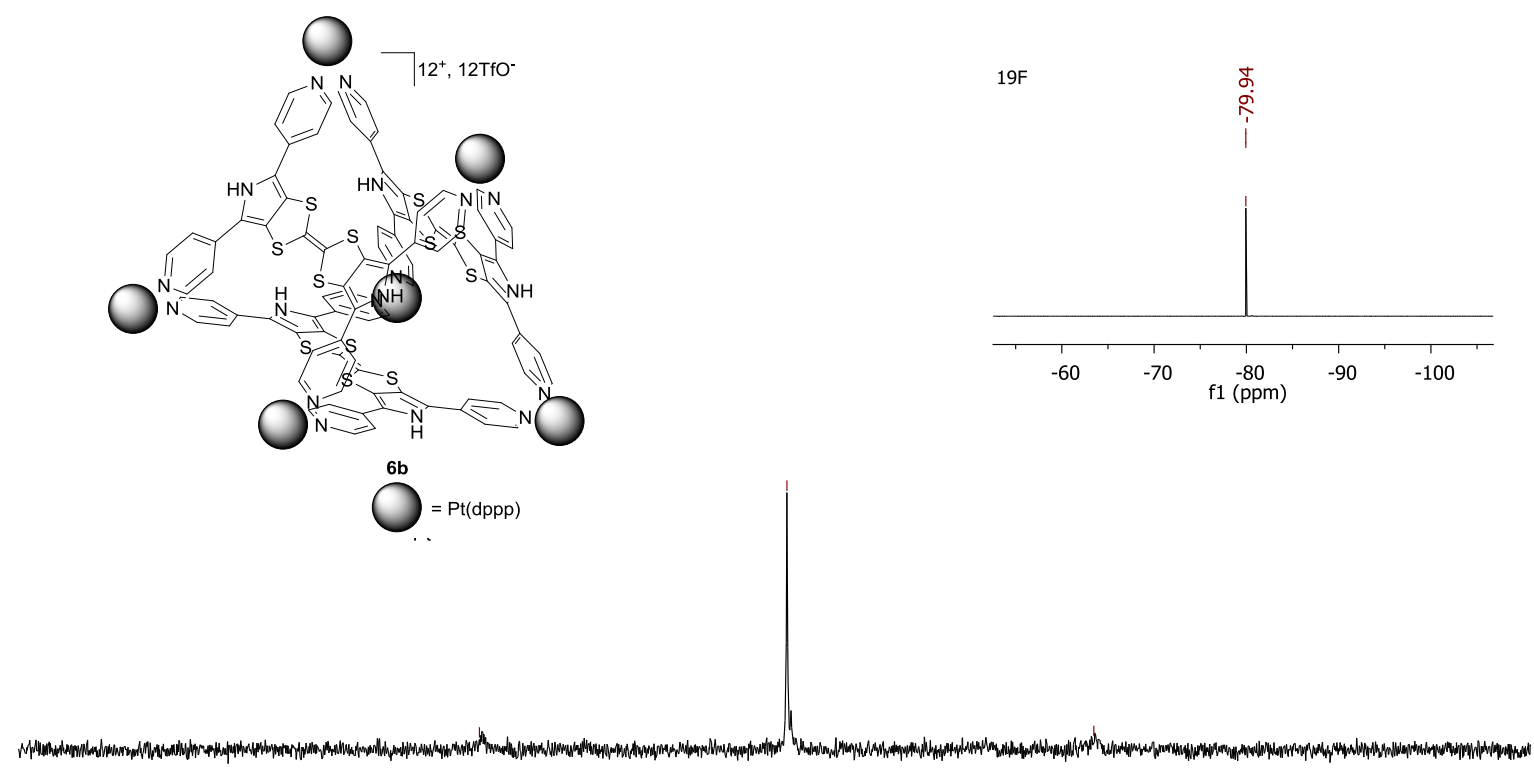

$\begin{array}{llllllllllllllll}16 & 14 & 12 & 10 & 8 & 6 & 4 & 2 & 0 & -2 & -4 & -6 & -8 & -10 & \begin{array}{l}-14 \\ \mathrm{fpm}\end{array}\end{array}$

Figure S178. ${ }^{31} \mathrm{P}$ and ${ }^{19} \mathrm{~F}$ NMR spectra of $\mathbf{6 b}$ in DMSO-d $\mathrm{d}_{6}$

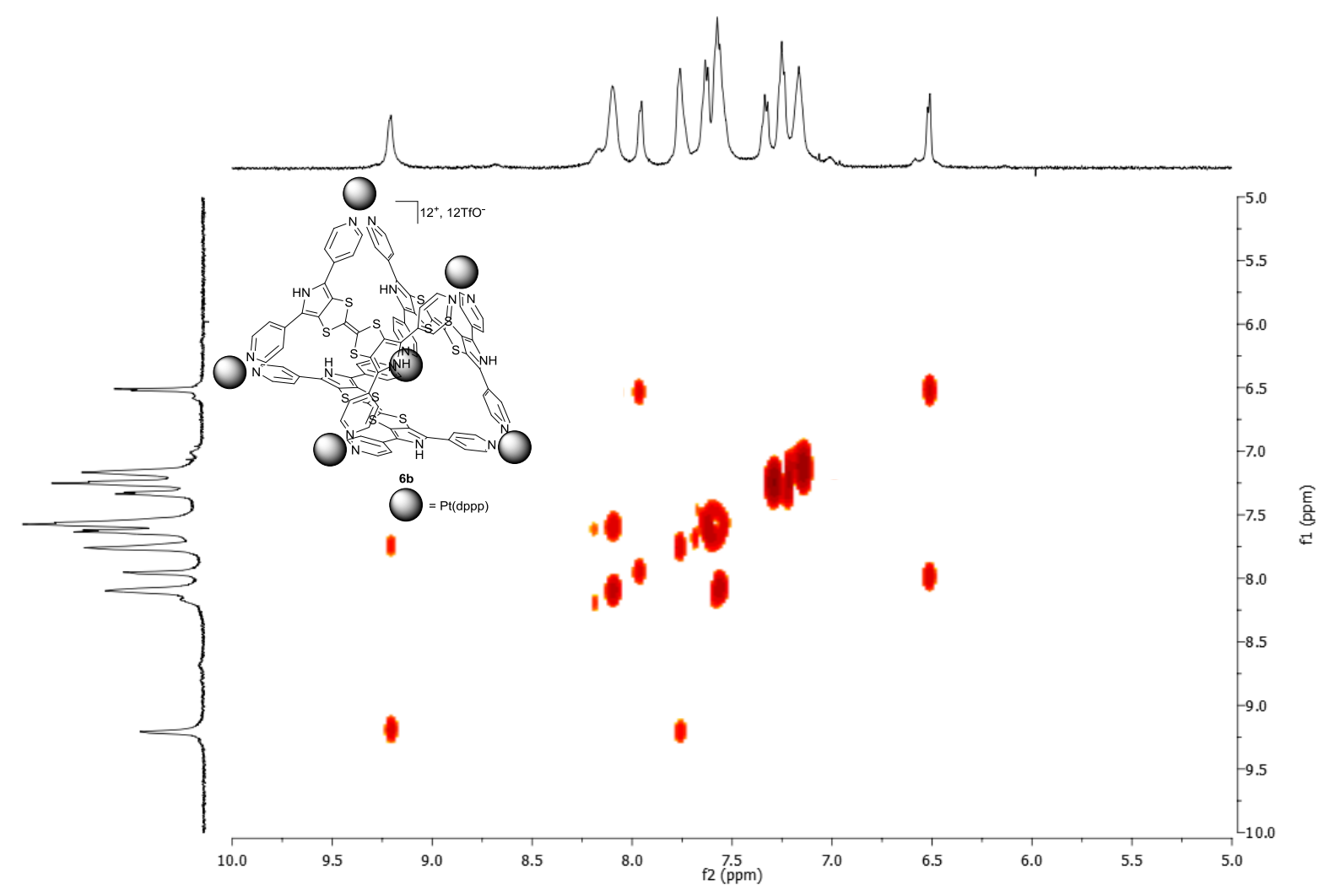

Figure S19. COSY NMR spectra of $6 \mathbf{b}$ in DMSO-d $\mathrm{d}_{6}$ 


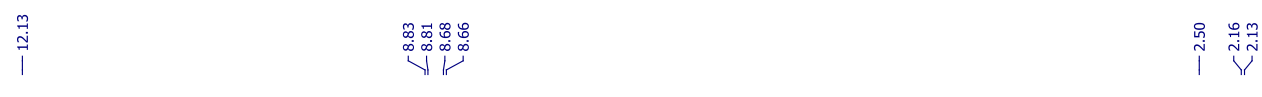

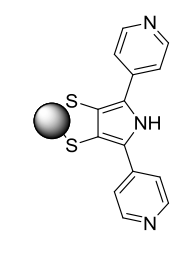

7a $\bigcirc=\operatorname{Pt}\left(\mathrm{PEt}_{3}\right)_{2}$

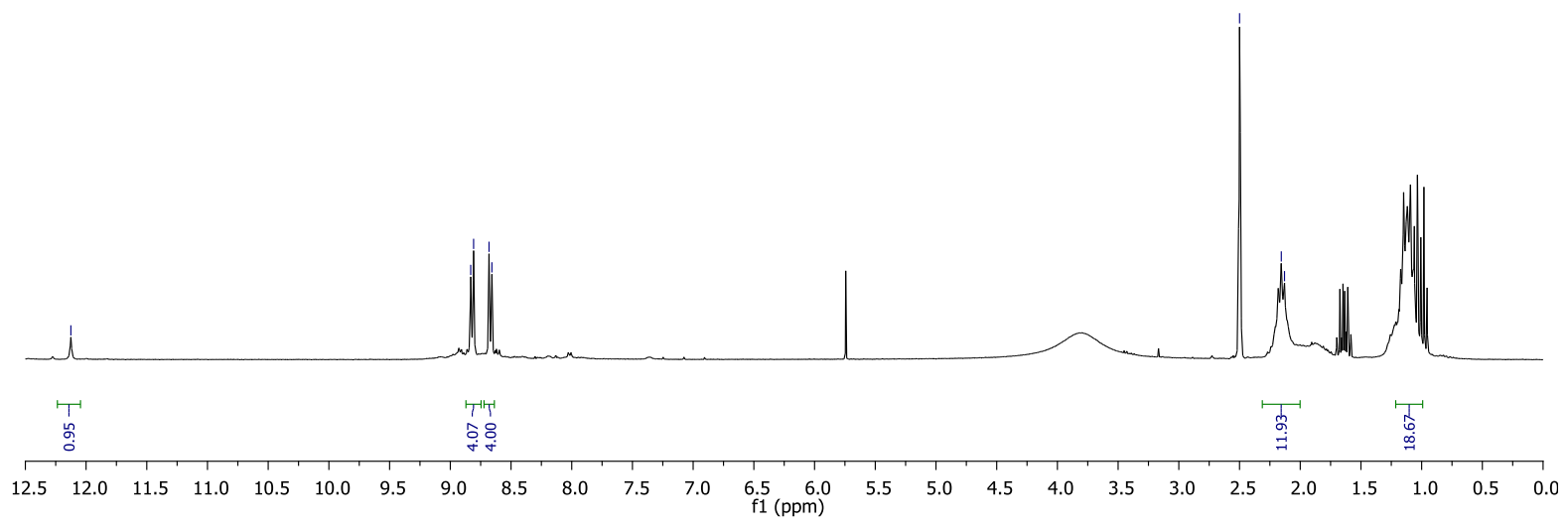

Figure S20. ${ }^{1} \mathrm{H}$ NMR spectrum of 7a in DMSO-d 6

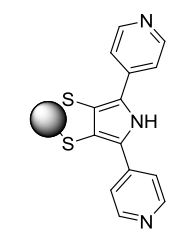

7a $\mathrm{O}=\mathrm{Pt}\left(\mathrm{PEt}_{3}\right)_{2}$

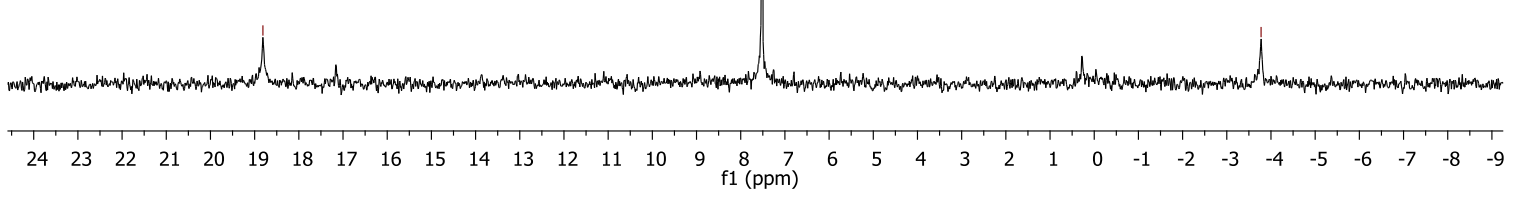

Figure S21. ${ }^{31} \mathrm{P}$ NMR spectrum of $\mathbf{7 a}$ in DMSO-d 6 


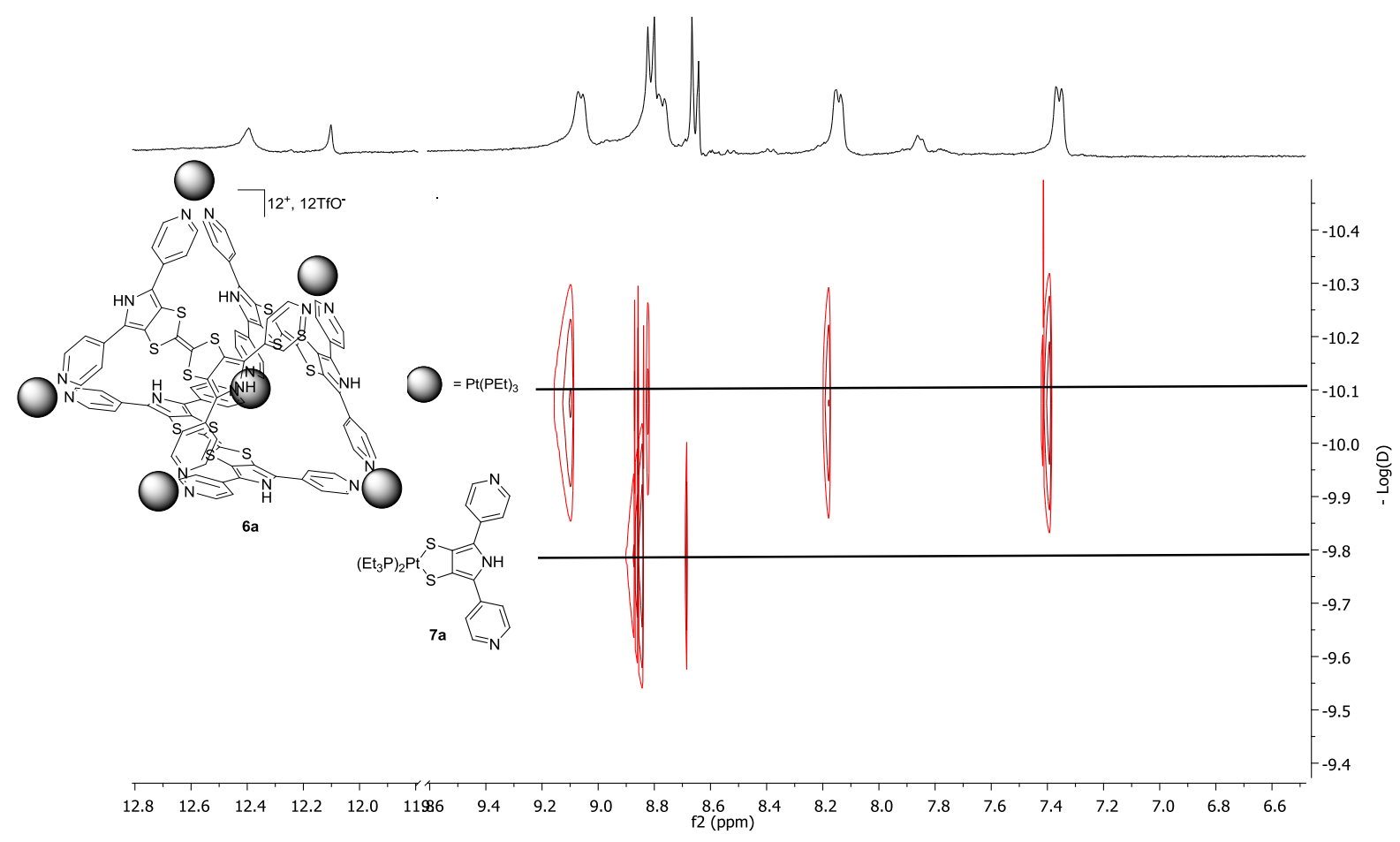

Figure S182. DOSY NMR monitoring of the conversion of $\mathbf{6 a}$ into $7 \mathbf{a}, \mathrm{DMSO}-\mathrm{d}_{6}, 3$ days at $75^{\circ} \mathrm{C}$

$\stackrel{\Re}{=}$

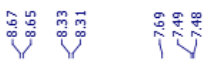
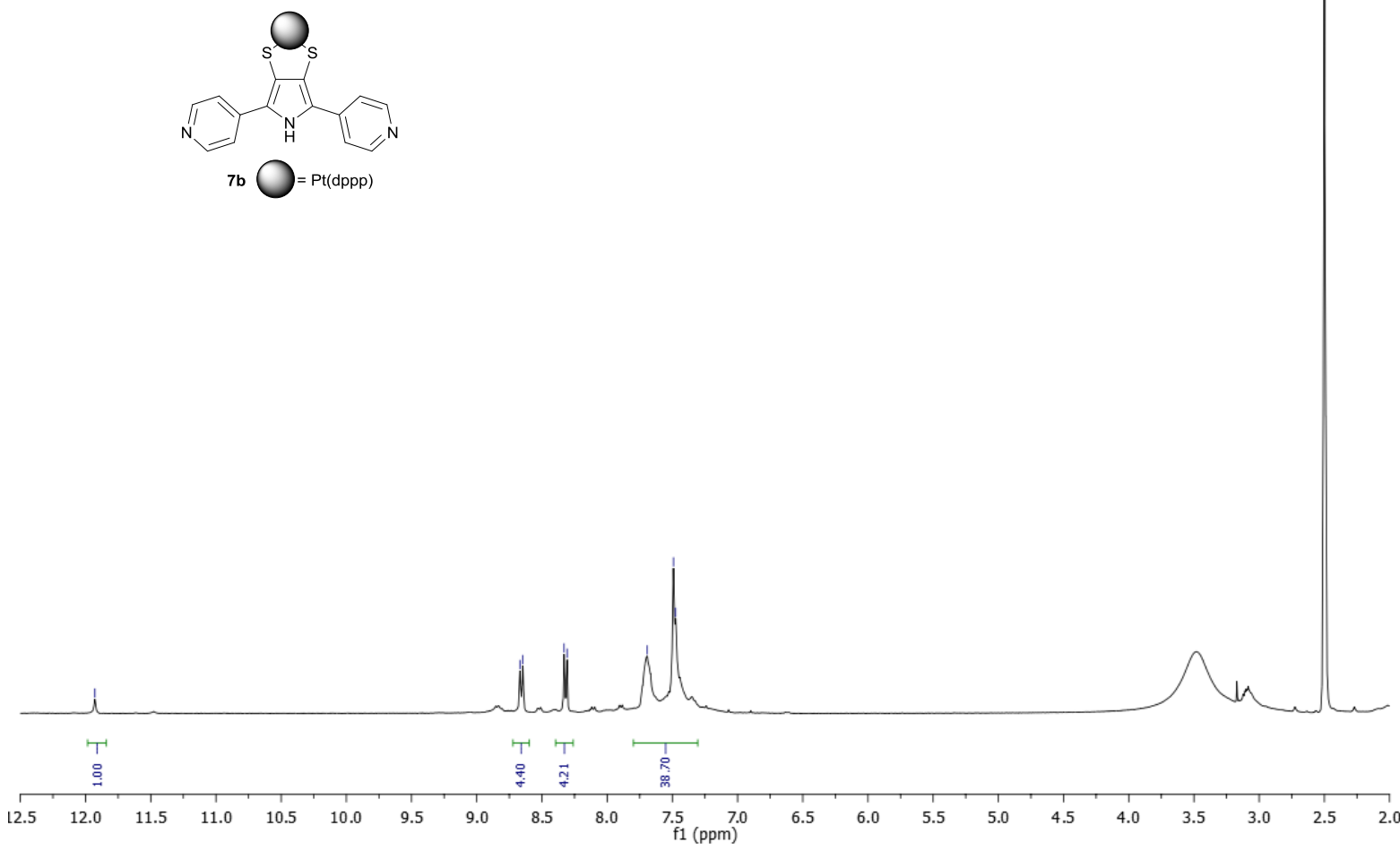

${ }^{1} \mathrm{H}$ spectra of $\mathbf{7 b}$ in DMSO- $\mathrm{d}_{6}$

Figure S23. 

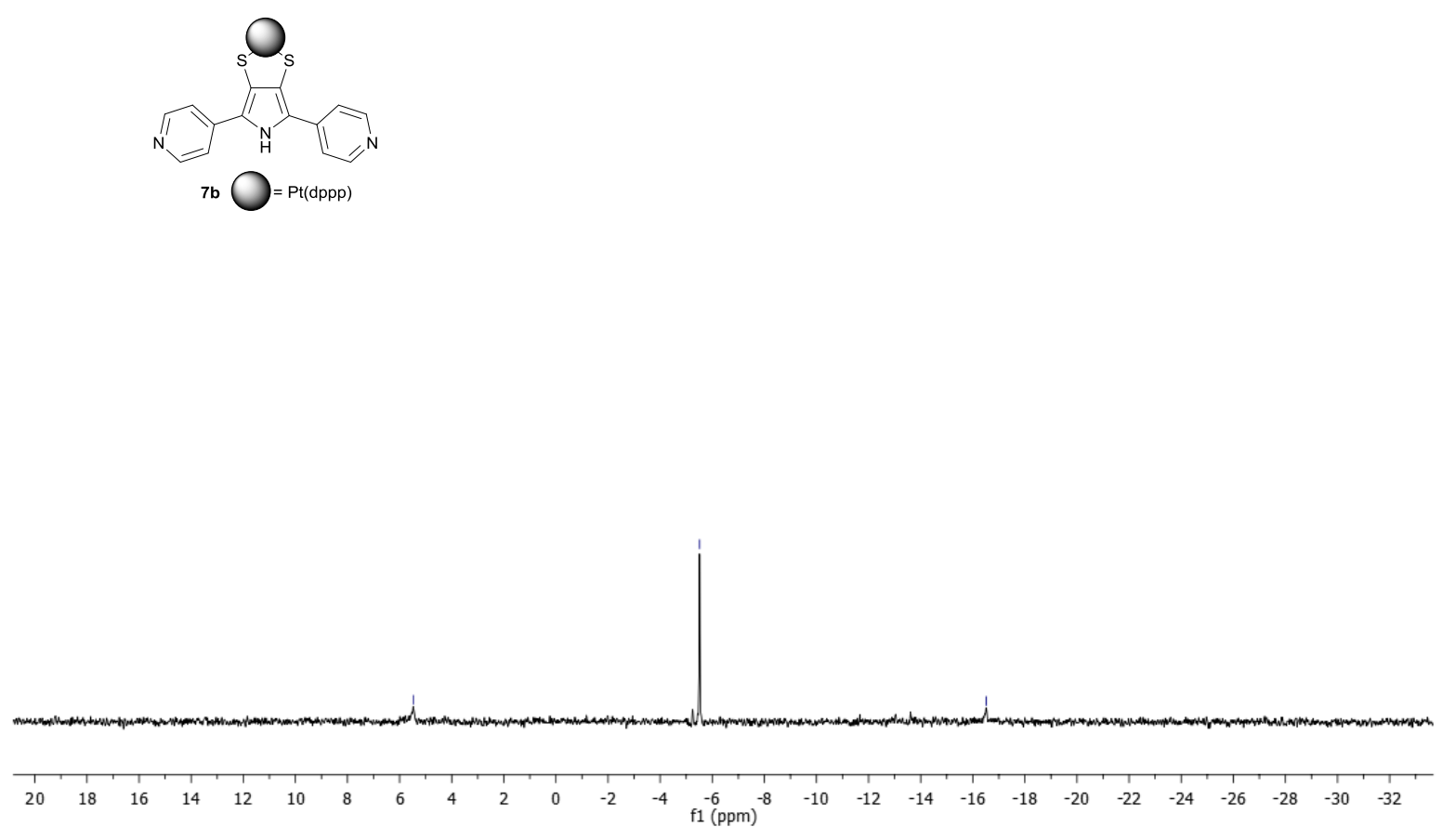

${ }^{31} \mathrm{P}$ spectra of $\mathbf{7 b}$ in DMSO- $\mathrm{d}_{6}$

Figure S194.

\section{Mass Spectra of 6a, 6b}

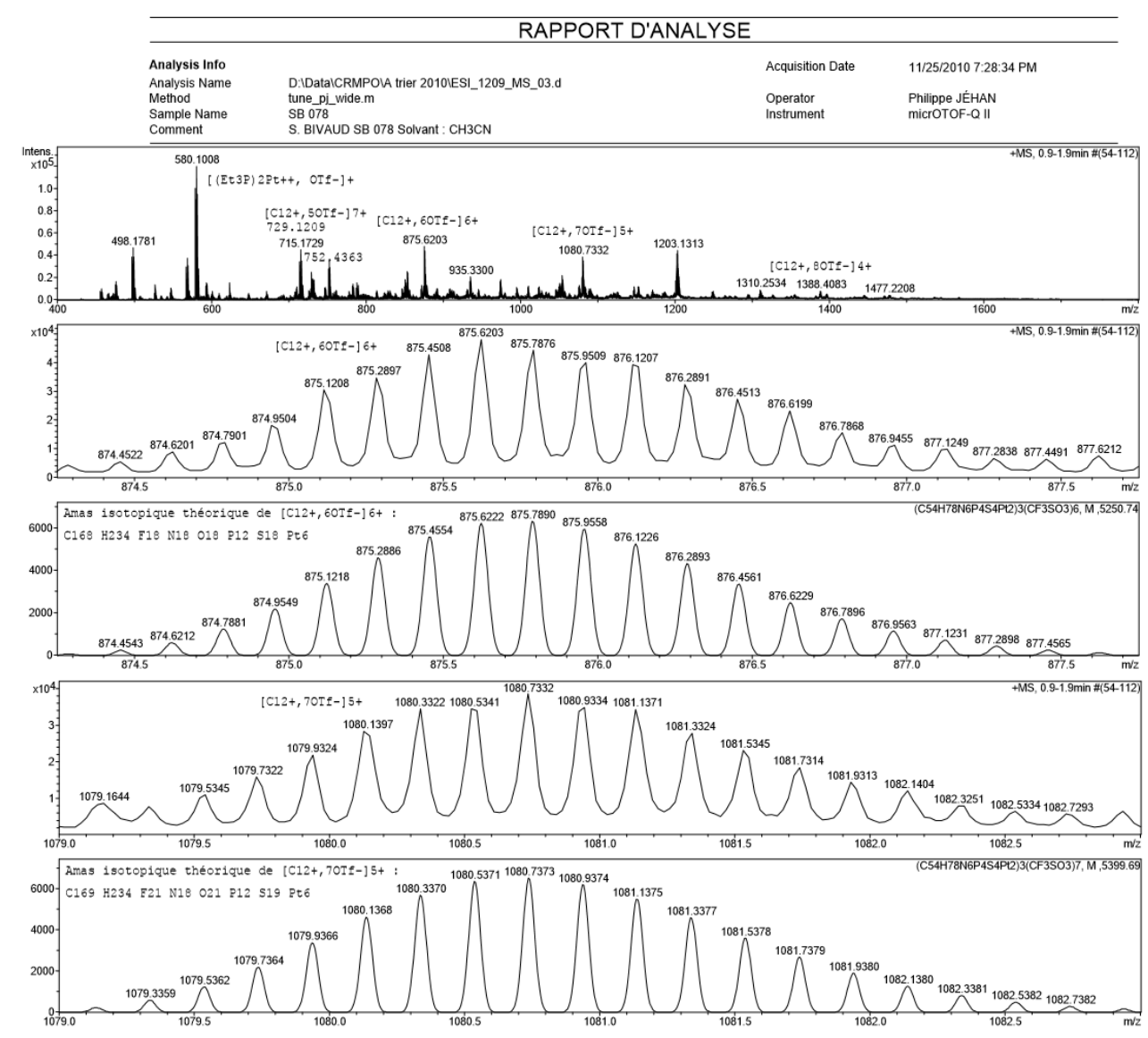


E;chempubsoc

Eur IC

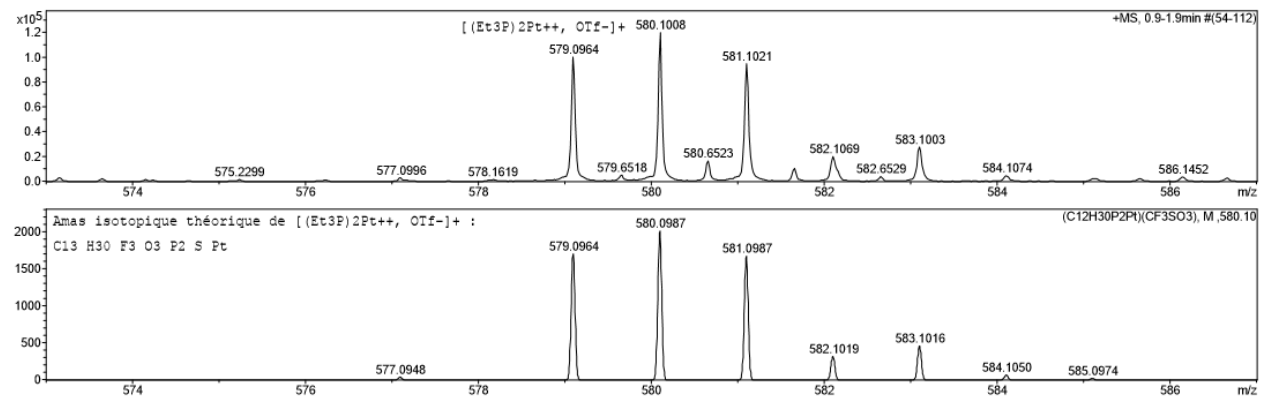

Figure S205. Mass spectrum of $\mathbf{6 a}$ 

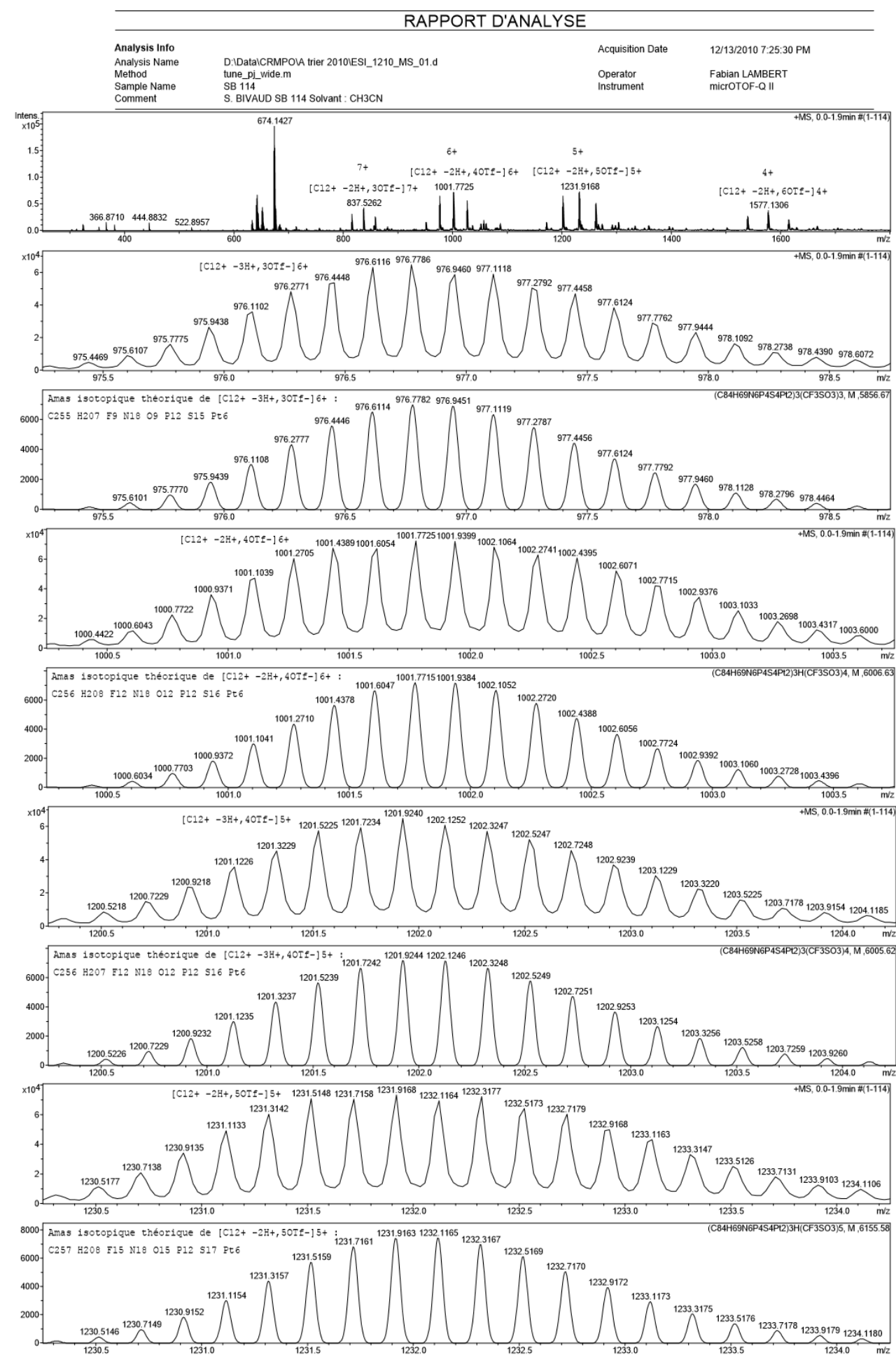

Figure S216. Mass spectrum of $\mathbf{6 b}$ 


\section{Cyclic Voltammetry}

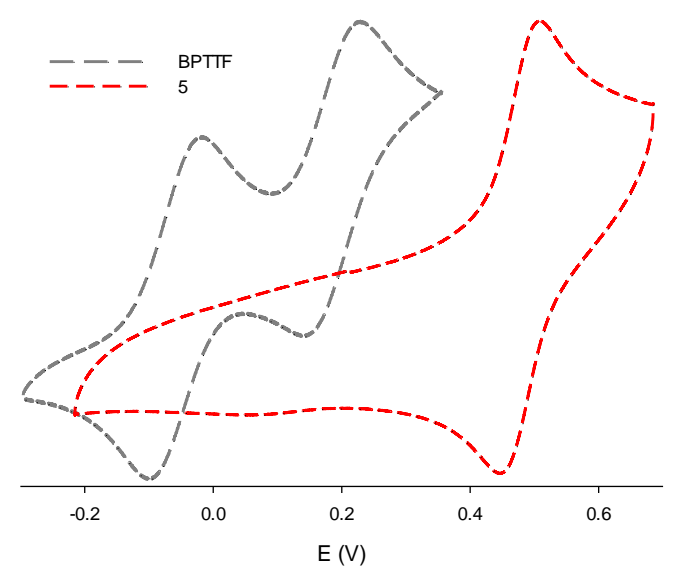

Figure S27. Red, cyclic voltammogram of $5\left(\mathrm{C}=6 \times 10^{-4}, \mathrm{CH}_{3} \mathrm{CN}, \mathrm{nBu}_{4} \mathrm{NPF}_{6}\right.$, working electrode: $\mathrm{C}_{\mathrm{gr}}$; black, BPTTF $(\mathrm{C}=5$ x $10^{-4} \mathrm{M}, \mathrm{DMF}, \mathrm{nBu}_{4} \mathrm{NPF}_{6}, \mathrm{Pt}$ ), vs Fc/Fc ${ }^{+}$ 


\section{X-Ray Structures}
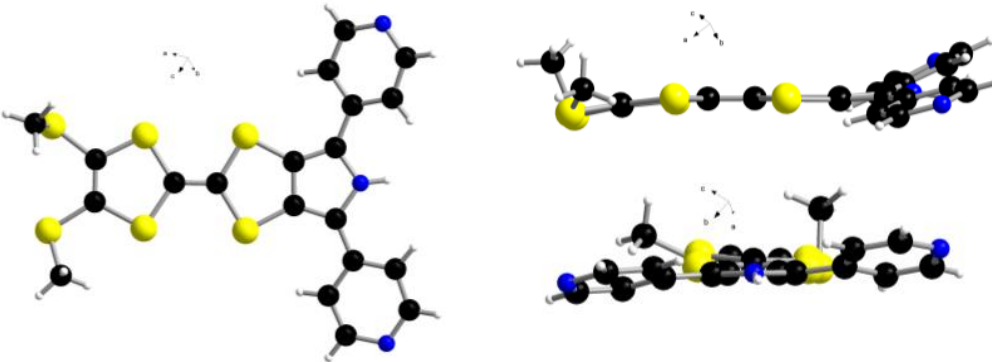

Figure S28. X-Ray structure of 2

Table S1. Crystal data and structure refinement for $\mathbf{2}$

Empirical formula
Formula weight
Temperature
Wavelength
Crystal system, space group
Unit cell dimensions
Volume
Z, Calculated density
Absorption coefficient
F(0oo)
Crystal size
Theta range for data collection
Limiting indices
Reflections collected / unique
Completeness to theta = 26.12
Absorption correction
Max. and min. transmission
Refinement method
Data / restraints / parameters
Goodness-of-fit on F^2
Final R indices [I>2sigma(I)]
R indices (all data)
Largest diff. peak and hole

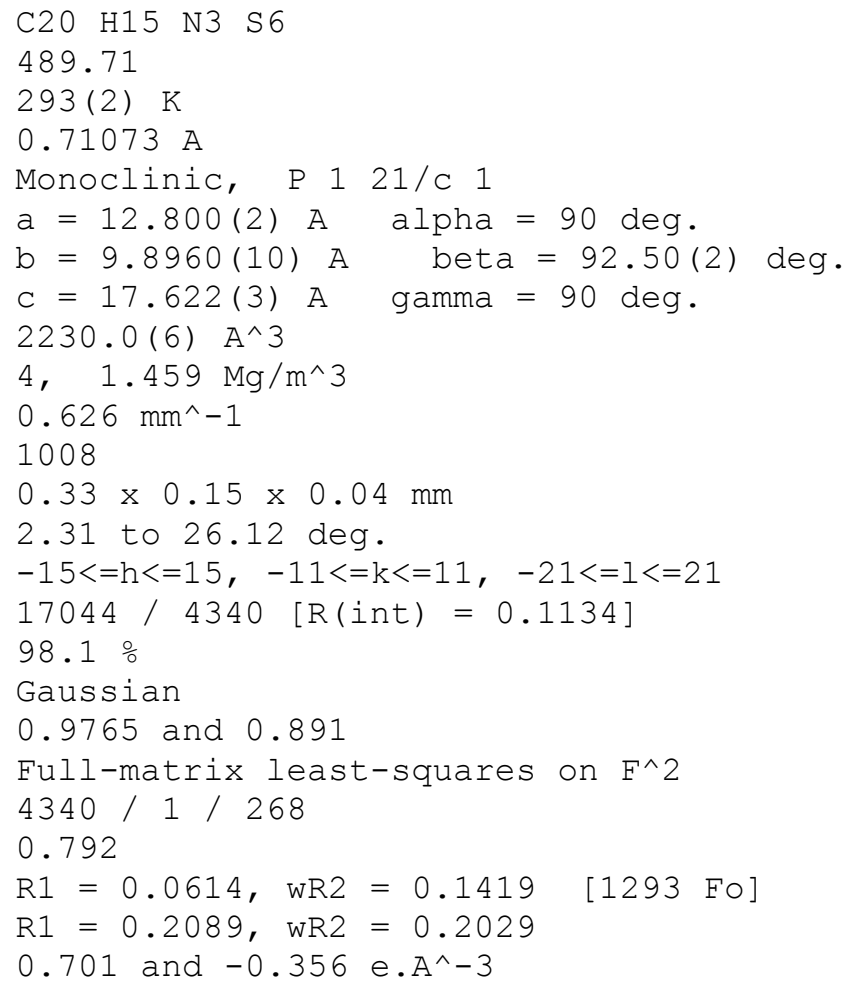

Table s2. Atomic coordinates and equivalent isotropic displacement parameters for 2 . $\mathrm{U}(\mathrm{eq})$ is defined as one third of the trace of the orthogonalized Uij tensor.

\begin{tabular}{lcccc}
\hline & $x$ & $y$ & $z$ & $U(e q)$ \\
\hline$C(01)$ & $0.8562(5)$ & $-0.0735(8)$ & $1.0455(4)$ & $0.067(2)$ \\
$C(02)$ & $0.9410(6)$ & $-0.1226(10)$ & $1.0851(4)$ & $0.082(3)$ \\
$C(03)$ & $1.0948(6)$ & $-0.1637(10)$ & $1.1835(5)$ & $0.079(2)$ \\
$C(04)$ & $1.1159(6)$ & $-0.2432(10)$ & $1.1236(5)$ & $0.081(2)$ \\
$C(05)$ & $1.2006(10)$ & $-0.4721(15)$ & $1.1649(8)$ & $0.171(6)$ \\
$C(06)$ & $1.1062(11)$ & $-0.088(2)$ & $1.3275(6)$ & $0.223(10)$ \\
$C(07)$ & $0.6944(5)$ & $0.0606(7)$ & $1.0010(3)$ & $0.0552(17)$ \\
$C(08)$ & $0.7149(5)$ & $-0.0296(8)$ & $0.9427(4)$ & $0.0571(18)$ \\
$C(09)$ & $0.6024(5)$ & $0.1296(7)$ & $0.9811(3)$ & $0.0531(17)$
\end{tabular}




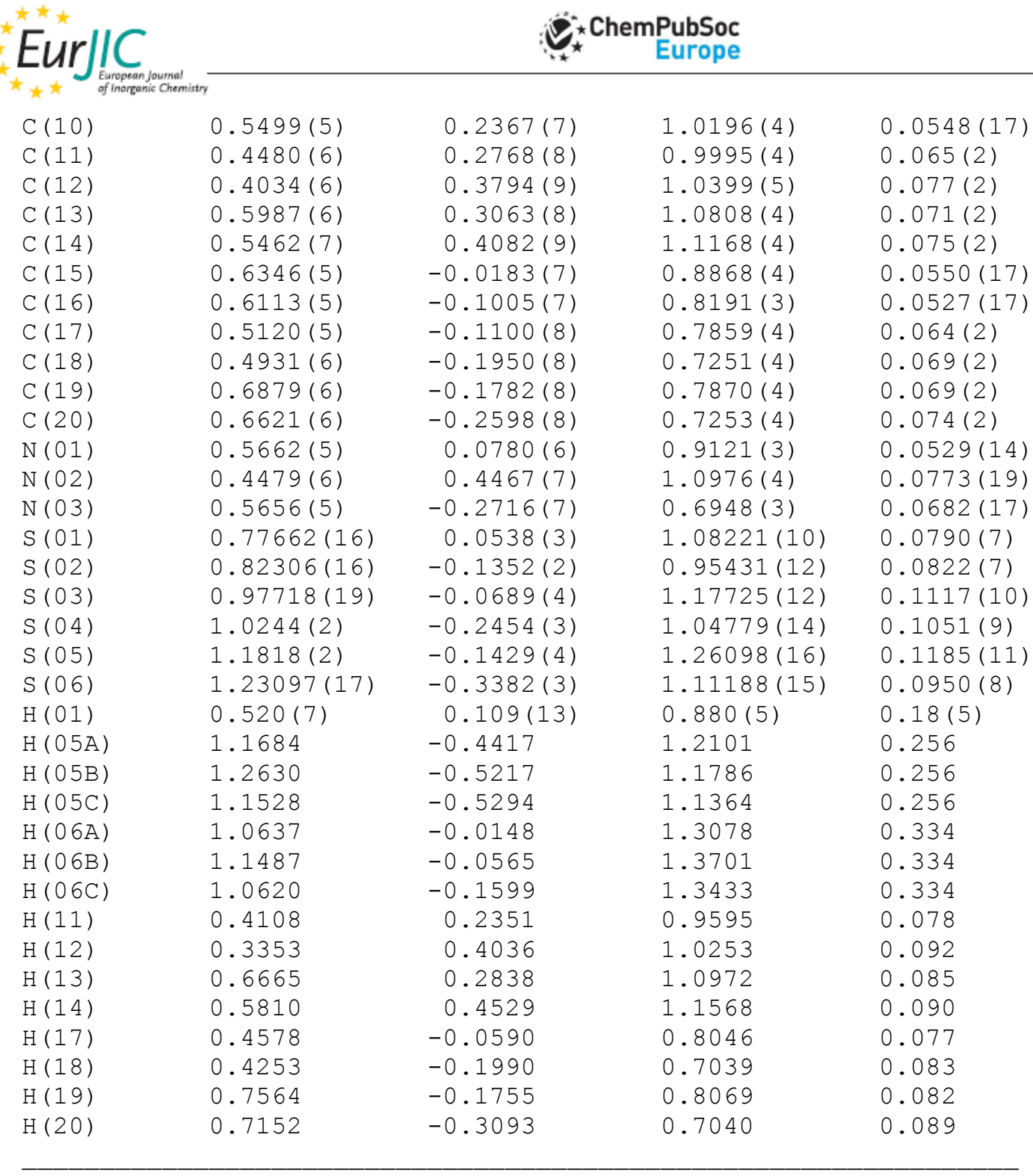

Table s3. Bond lengths [A] for 2.

\begin{tabular}{ll}
\hline$C(01)-C(02)$ & $1.354(9)$ \\
$C(01)-S(02)$ & $1.754(7)$ \\
$C(01)-S(01)$ & $1.761(8)$ \\
$C(02)-S(03)$ & $1.752(8)$ \\
$C(02)-S(04)$ & $1.763(9)$ \\
$C(03)-C(04)$ & $1.352(11)$ \\
$C(03)-S(05)$ & $1.737(8)$ \\
$C(03)-S(03)$ & $1.773(8)$ \\
$C(04)-S(04)$ & $1.738(8)$ \\
$C(04)-S(06)$ & $1.767(9)$ \\
$C(05)-S(06)$ & $1.677(12)$ \\
$C(05)-H(05 A)$ & 0.9600 \\
$C(05)-H(05 B)$ & 0.9600 \\
$C(05)-H(05 C)$ & 0.9600 \\
$C(06)-S(05)$ & $1.646(13)$ \\
$C(06)-H(06 A)$ & 0.9600 \\
$C(06)-H(06 B)$ & 0.9600 \\
$C(06)-H(06 C)$ & 0.9600 \\
$C(07)-C(08)$ & $1.393(9)$ \\
$C(07)-C(09)$ & $1.393(9)$ \\
$C(07)-S(01)$ & $1.741(6)$ \\
$C(08)-C(15)$ & $1.396(8)$
\end{tabular}




$\begin{array}{ll}\mathrm{C}(08)-\mathrm{S}(02) & 1.740(7) \\ \mathrm{C}(09)-\mathrm{N}(01) & 1.381(8) \\ \mathrm{C}(09)-\mathrm{C}(10) & 1.440(9) \\ \mathrm{C}(10)-\mathrm{C}(11) & 1.394(9) \\ \mathrm{C}(10)-\mathrm{C}(13) & 1.404(9) \\ \mathrm{C}(11)-\mathrm{C}(12) & 1.378(10) \\ \mathrm{C}(11)-\mathrm{H}(11) & 0.9300 \\ \mathrm{C}(12)-\mathrm{N}(02) & 1.323(10) \\ \mathrm{C}(12)-\mathrm{H}(12) & 0.9300 \\ \mathrm{C}(13)-\mathrm{C}(14) & 1.381(10) \\ \mathrm{C}(13)-\mathrm{H}(13) & 0.9300 \\ \mathrm{C}(14)-\mathrm{N}(02) & 1.343(10) \\ \mathrm{C}(14)-\mathrm{H}(14) & 0.9300 \\ \mathrm{C}(15)-\mathrm{N}(01) & 1.381(9) \\ \mathrm{C}(15)-\mathrm{C}(16) & 1.464(9) \\ \mathrm{C}(16)-\mathrm{C}(17) & 1.380(8) \\ \mathrm{C}(16)-\mathrm{C}(19) & 1.386(9) \\ \mathrm{C}(17)-\mathrm{C}(18) & 1.374(10) \\ \mathrm{C}(17)-\mathrm{H}(17) & 0.9300 \\ \mathrm{C}(18)-\mathrm{N}(03) & 1.329(9) \\ \mathrm{C}(18)-\mathrm{H}(18) & 0.9300 \\ \mathrm{C}(19)-\mathrm{C}(20) & 1.383(10) \\ \mathrm{C}(19)-\mathrm{H}(19) & 0.9300 \\ \mathrm{C}(20)-\mathrm{N}(03) & 1.331(9) \\ \mathrm{C}(20)-\mathrm{H}(20) & 0.9300 \\ \mathrm{~N}(01)-\mathrm{H}(01) & 0.860(10) \\ & \end{array}$

Table S4. Bond angles [deg] for 2.

\begin{tabular}{ll}
\hline $\mathrm{C}(02)-\mathrm{C}(01)-\mathrm{S}(02)$ & $120.2(6)$ \\
$\mathrm{C}(02)-\mathrm{C}(01)-\mathrm{S}(01)$ & $122.1(6)$ \\
$\mathrm{S}(02)-\mathrm{C}(01)-\mathrm{S}(01)$ & $117.7(4)$ \\
$\mathrm{C}(01)-\mathrm{C}(02)-\mathrm{S}(03)$ & $122.9(6)$ \\
$\mathrm{C}(01)-\mathrm{C}(02)-\mathrm{S}(04)$ & $122.7(6)$ \\
$\mathrm{S}(03)-\mathrm{C}(02)-\mathrm{S}(04)$ & $114.5(4)$ \\
$\mathrm{C}(04)-\mathrm{C}(03)-\mathrm{S}(05)$ & $122.7(6)$ \\
$\mathrm{C}(04)-\mathrm{C}(03)-\mathrm{S}(03)$ & $117.2(6)$ \\
$\mathrm{S}(05)-\mathrm{C}(03)-\mathrm{S}(03)$ & $120.0(6)$ \\
$\mathrm{C}(03)-\mathrm{C}(04)-\mathrm{S}(04)$ & $117.1(6)$ \\
$\mathrm{C}(03)-\mathrm{C}(04)-\mathrm{S}(06)$ & $126.6(6)$ \\
$\mathrm{S}(04)-\mathrm{C}(04)-\mathrm{S}(06)$ & $116.1(6)$ \\
$\mathrm{S}(06)-\mathrm{C}(05)-\mathrm{H}(05 \mathrm{~A})$ & 109.5 \\
$\mathrm{~S}(06)-\mathrm{C}(05)-\mathrm{H}(05 \mathrm{~B})$ & 109.5 \\
$\mathrm{H}(05 \mathrm{~A})-\mathrm{C}(05)-\mathrm{H}(05 \mathrm{~B})$ & 109.5 \\
$\mathrm{~S}(06)-\mathrm{C}(05)-\mathrm{H}(05 \mathrm{C})$ & 109.5 \\
$\mathrm{H}(05 \mathrm{~A})-\mathrm{C}(05)-\mathrm{H}(05 \mathrm{C})$ & 109.5 \\
$\mathrm{H}(05 \mathrm{~B})-\mathrm{C}(05)-\mathrm{H}(05 \mathrm{C})$ & 109.5 \\
$\mathrm{~S}(05)-\mathrm{C}(06)-\mathrm{H}(06 \mathrm{~A})$ & 109.5 \\
$\mathrm{~S}(05)-\mathrm{C}(06)-\mathrm{H}(06 \mathrm{~B})$ & 109.5 \\
$\mathrm{H}(06 \mathrm{~A})-\mathrm{C}(06)-\mathrm{H}(06 \mathrm{~B})$ & 109.5 \\
$\mathrm{~S}(05)-\mathrm{C}(06)-\mathrm{H}(06 \mathrm{C})$ & 109.5 \\
$\mathrm{H}(06 \mathrm{~A})-\mathrm{C}(06)-\mathrm{H}(06 \mathrm{C})$ & 109.5 \\
$\mathrm{H}(06 \mathrm{~B})-\mathrm{C}(06)-\mathrm{H}(06 \mathrm{C})$ & 109.5 \\
$\mathrm{C}(08)-\mathrm{C}(07)-\mathrm{C}(09)$ & $108.2(6)$ \\
$\mathrm{C}(08)-\mathrm{C}(07)-\mathrm{S}(01)$ & $117.1(5)$ \\
$\mathrm{C}(09)-\mathrm{C}(07)-\mathrm{S}(01)$ & $134.4(5)$ \\
$\mathrm{C}(07)-\mathrm{C}(08)-\mathrm{C}(15)$ & $108.2(6)$ \\
$\mathrm{C}(07)-\mathrm{C}(08)-\mathrm{S}(02)$ & $118.1(5)$ \\
$\mathrm{C}(15)-\mathrm{C}(08)-\mathrm{S}(02)$ & $133.5(6)$ \\
$\mathrm{N}(01)-\mathrm{C}(09)-\mathrm{C}(07)$ & $106.7(6)$ \\
$\mathrm{N}(01)-\mathrm{C}(09)-\mathrm{C}(10)$ & $122.8(6)$ \\
&
\end{tabular}




\begin{tabular}{|c|c|}
\hline$C(07)-C(09)-C(10)$ & $130.5(6)$ \\
\hline$C(11)-C(10)-C(13)$ & $115.8(7)$ \\
\hline$C(11)-C(10)-C(09)$ & $122.7(6)$ \\
\hline$C(13)-C(10)-C(09)$ & $121.5(6)$ \\
\hline$C(12)-C(11)-C(10)$ & $118.9(7)$ \\
\hline $\mathrm{C}(12)-\mathrm{C}(11)-\mathrm{H}(11)$ & 120.6 \\
\hline $\mathrm{C}(10)-\mathrm{C}(11)-\mathrm{H}(11)$ & 120.6 \\
\hline $\mathrm{N}(02)-\mathrm{C}(12)-\mathrm{C}(11)$ & $126.4(8$ \\
\hline $\mathrm{N}(02)-\mathrm{C}(12)-\mathrm{H}(12)$ & 116.8 \\
\hline $\mathrm{C}(11)-\mathrm{C}(12)-\mathrm{H}(12)$ & 16.8 \\
\hline$C(14)-C(13)-C(10)$ & $120.2(7)$ \\
\hline$C(14)-C(13)-H(13)$ & 119.9 \\
\hline $\mathrm{C}(10)-\mathrm{C}(13)-\mathrm{H}(13)$ & 9.9 \\
\hline$N(02)-C(14)-C(13)$ & $124.1(8)$ \\
\hline $\mathrm{N}(02)-\mathrm{C}(14)-\mathrm{H}(14)$ & 118.0 \\
\hline $\mathrm{C}(13)-\mathrm{C}(14)-\mathrm{H}(14)$ & 18.0 \\
\hline $\mathrm{N}(01)-\mathrm{C}(15)-\mathrm{C}(08)$ & $106.5(6)$ \\
\hline $\mathrm{N}(01)-\mathrm{C}(15)-\mathrm{C}(16)$ & $122.5(6)$ \\
\hline$C(08)-C(15)-C(16)$ & $30.4(7)$ \\
\hline$C(17)-C(16)-C(19)$ & $116.4(6)$ \\
\hline$C(17)-C(16)-C(15)$ & $122.3(6)$ \\
\hline$C(19)-C(16)-C(15)$ & $1.2(6)$ \\
\hline$C(18)-C(17)-C(16)$ & $120.0(7)$ \\
\hline $\mathrm{C}(18)-\mathrm{C}(17)-\mathrm{H}(17)$ & 120.0 \\
\hline $\mathrm{C}(16)-\mathrm{C}(17)-\mathrm{H}(17)$ & 120.0 \\
\hline$N(03)-C(18)-C(17)$ & $124.1(7)$ \\
\hline $\mathrm{N}(03)-\mathrm{C}(18)-\mathrm{H}(18)$ & 117.9 \\
\hline $\mathrm{C}(17)-\mathrm{C}(18)-\mathrm{H}(18)$ & 117.9 \\
\hline$C(20)-C(19)-C(16)$ & $119.7(6)$ \\
\hline $\mathrm{C}(20)-\mathrm{C}(19)-\mathrm{H}(19)$ & 120.1 \\
\hline $\mathrm{C}(16)-\mathrm{C}(19)-\mathrm{H}(19)$ & 120.1 \\
\hline$N(03)-C(20)-C(19)$ & $123.7(8)$ \\
\hline $\mathrm{N}(03)-\mathrm{C}(20)-\mathrm{H}(20)$ & 118.1 \\
\hline $\mathrm{C}(19)-\mathrm{C}(20)-\mathrm{H}(20)$ & 118.1 \\
\hline $\mathrm{C}(09)-\mathrm{N}(01)-\mathrm{C}(15)$ & $110.3(6)$ \\
\hline $\mathrm{C}(09)-\mathrm{N}(01)-\mathrm{H}(01)$ & $130(9)$ \\
\hline $\mathrm{C}(15)-\mathrm{N}(01)-\mathrm{H}(01)$ & $118(9)$ \\
\hline$C(12)-N(02)-C(14)$ & $114.7(7)$ \\
\hline$C(18)-N(03)-C(20)$ & $116.0(6)$ \\
\hline$C(07)-S(01)-C(01)$ & $93.7(3)$ \\
\hline$C(08)-S(02)-C(01)$ & $93.3(3$ \\
\hline$C(02)-S(03)-C(03)$ & $95.0(4)$ \\
\hline$C(04)-S(04)-C(02)$ & $95.9(4$ \\
\hline$C(06)-S(05)-C(03)$ & $102.9(5$ \\
\hline$C(05)-S(06)-C(04)$ & $98 \quad 115$ \\
\hline
\end{tabular}

Table s5. Anisotropic displacement parameters for 2.

The anisotropic displacement factor exponent takes the form: $-2 \mathrm{pi}^{\wedge} 2$ [ $\left.\mathrm{h}^{\wedge} 2 \mathrm{a}^{\star \wedge} 2 \mathrm{U} 11+\ldots+2 \mathrm{~h} k \mathrm{a}^{\star} \mathrm{b} \mathrm{b}^{\star} \mathrm{U} 12\right]$

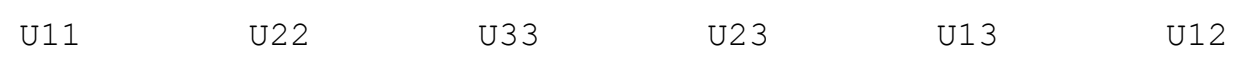

$\begin{array}{lllllll}\overline{\mathrm{C}(01)} & 0.060(4) & 0.077(6) & 0.065(4) & 0.003(4) & -0.011(3) & 0.016(4)\end{array}$




\begin{tabular}{|c|c|c|c|c|c|c|}
\hline$C(02)$ & $0.066(5)$ & $0.104(7)$ & $0.074(5)$ & $0.006(5)$ & $-0.012(4)$ & $0.026(5)$ \\
\hline$C(03)$ & $0.067(5)$ & $0.097(7)$ & $0.070(5)$ & $0.014(5)$ & $-0.016(4)$ & $0.008(5)$ \\
\hline$C(04)$ & $0.057(5)$ & $0.092(7)$ & $0.094(6)$ & $0.017(5)$ & $-0.015(4)$ & $0.014(4)$ \\
\hline$C(05)$ & $0.121(10)$ & $0.211(16)$ & $0.184(13)$ & $0.085(12)$ & $0.058(9)$ & $0.003(10)$ \\
\hline$C(06)$ & $0.176(12)$ & $0.38(3)$ & $0.102(8)$ & $-0.074(12)$ & $-0.087(9)$ & $0.158(15)$ \\
\hline$C(07)$ & $0.068(4)$ & $0.054(5)$ & $0.044(3)$ & $0.003(3)$ & $-0.003(3)$ & $0.004(4)$ \\
\hline$C(08)$ & $0.054(4)$ & $0.065(5)$ & $0.052(4)$ & $0.002(3)$ & $-0.008(3)$ & $0.008(3)$ \\
\hline$C(09)$ & $0.057(4)$ & $0.053(5)$ & $0.049(4)$ & $0.004(3)$ & $-0.005(3)$ & $0.001(4)$ \\
\hline$C(10)$ & $0.064(4)$ & $0.048(5)$ & $0.052(4)$ & $0.013(3)$ & $-0.001(3)$ & $0.003(4)$ \\
\hline$C(11)$ & $0.065(5)$ & $0.069(6)$ & $0.060(4)$ & $0.000(4)$ & $0.002(3)$ & $0.005(4)$ \\
\hline$C(12)$ & $0.073(5)$ & $0.082(7)$ & $0.075(5)$ & $-0.004(5)$ & $0.001(4)$ & $0.014(5)$ \\
\hline$C(13)$ & $0.076(5)$ & $0.064(6)$ & $0.070(5)$ & $-0.005(4)$ & $-0.010(4)$ & $0.004(4)$ \\
\hline$C(14)$ & $0.084(6)$ & $0.063(6)$ & $0.079(5)$ & $-0.014(4)$ & $0.002(4)$ & $0.007(5)$ \\
\hline$C(15)$ & $0.065(4)$ & $0.049(5)$ & $0.050(4)$ & $0.001(3)$ & $-0.004(3)$ & $0.000(4)$ \\
\hline$C(16)$ & $0.058(4)$ & $0.054(5)$ & $0.046(3)$ & $-0.005(3)$ & $-0.008(3)$ & $0.000(3)$ \\
\hline$C(17)$ & $0.061(4)$ & $0.069(6)$ & $0.060(4)$ & $-0.002(4)$ & $-0.010(3)$ & $0.000(4)$ \\
\hline$C(18)$ & $0.075(5)$ & $0.068(6)$ & $0.062(4)$ & $-0.003(4)$ & $-0.022(4)$ & $-0.012(5)$ \\
\hline$C(19)$ & $0.060(4)$ & $0.081(6)$ & $0.063(4)$ & $-0.017(4)$ & $-0.013(3)$ & $0.003(4)$ \\
\hline$C(20)$ & $0.074(5)$ & $0.078(6)$ & $0.068(5)$ & $-0.011(4)$ & $-0.015(4)$ & $0.007(4)$ \\
\hline $\mathrm{N}(01)$ & $0.061(3)$ & $0.048(4)$ & $0.049(3)$ & $0.000(3)$ & $-0.005(3)$ & $0.002(3)$ \\
\hline$N(02)$ & $0.082(5)$ & $0.066(5)$ & $0.084(4)$ & $-0.005(4)$ & $0.008(4)$ & $0.013(4)$ \\
\hline$N(03)$ & $0.085(5)$ & $0.063(5)$ & $0.056(3)$ & $-0.008(3)$ & $-0.008(3)$ & $-0.005(4)$ \\
\hline$S(01)$ & $0.0747(13)$ & $0.1015(18)$ & $0.0587(11)$ & $-0.0145(11$ & $-0.0206(9)$ & $0.0249(12)$ \\
\hline$S(02)$ & $0.0759(14)$ & $0.0926(18)$ & $0.0761(13)$ & $-0.0176(12)$ & $-0.0202(10)$ & $0.0297(12)$ \\
\hline$S(03)$ & $0.0860(16)$ & $0.178(3)$ & $0.0686(13)$ & $-0.0117(15)$ & $-0.0248(11)$ & $0.0463(17)$ \\
\hline$S(04)$ & $0.0876(16)$ & $0.133(2)$ & $0.0924(16)$ & $-0.0106(15)$ & $-0.0203(12)$ & $0.0451(15)$ \\
\hline$S(05)$ & $0.0933(18)$ & $0.152(3)$ & 0.1061 (19) & $0.0004(18)$ & $-0.0402(15)$ & $0.0184(18)$ \\
\hline$S(06)$ & $0.0703(14)$ & $0.102(2)$ & $0.1128(18)$ & $0.0199(15)$ & $-0.0015(12)$ & $0.0150(13)$ \\
\hline
\end{tabular}



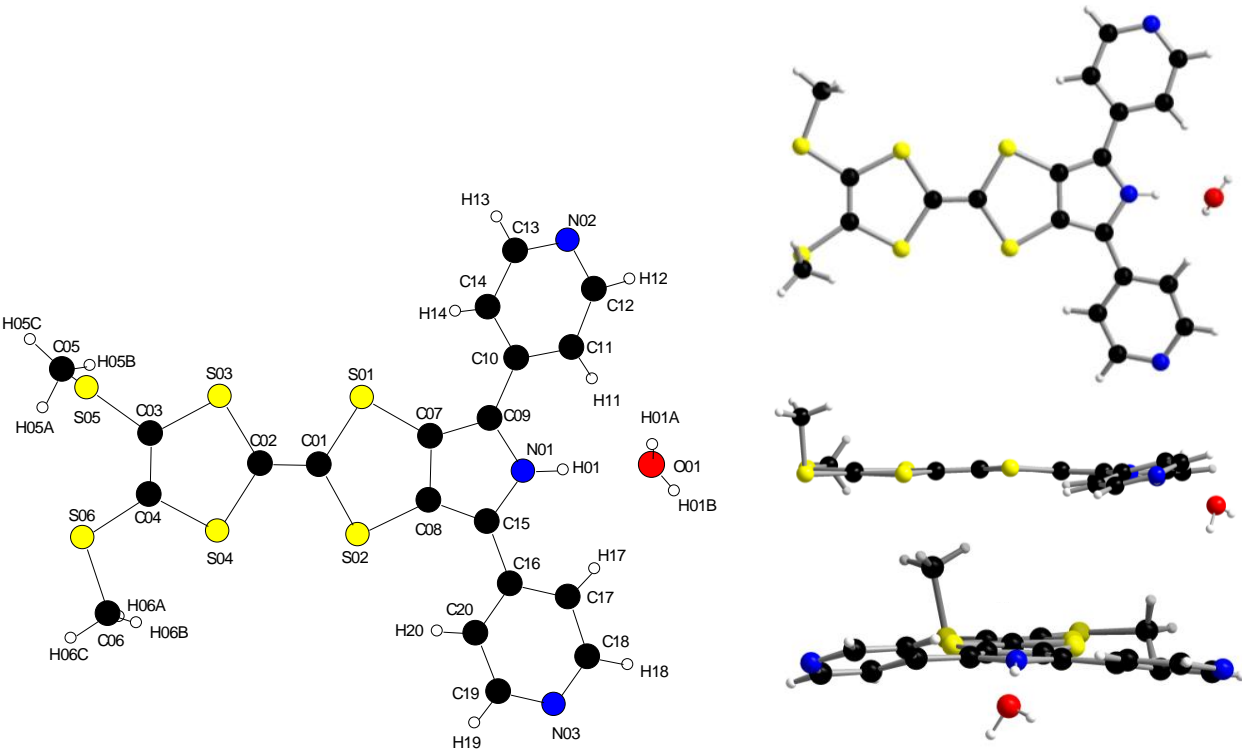

Figure S29. X-Ray structure of $\mathbf{2 . H 2 O}$

Table S6. Crystal data and structure refinement for 2.H20.

Empirical formula

Formula weight

$\mathrm{C} 20 \mathrm{H} 17$ N3 0 S6

Temperature

507.73

Wavelength

Crystal system, space group

Unit cell dimensions

293(2) K

$0.71073 \mathrm{~A}$

Monoclinic, $P 21 / \mathrm{n}$

$\mathrm{a}=14.0150(10) \mathrm{A}$ alpha $=90 \mathrm{deg}$.

$\mathrm{b}=5.4489(5) \mathrm{A}$ beta $=100.480(7) \mathrm{deg}$.

c $=30.219(2)$ A gamma $=90 \mathrm{deg}$.

Volume

$2269.2(3) A^{\wedge} 3$

Z, Calculated density

Absorption coefficient

$\mathrm{F}(000)$

$4, \quad 1.486 \mathrm{Mg} / \mathrm{m}^{\wedge} 3$

$0.621 \mathrm{~mm}^{\wedge}-1$

1048

Crystal size

Theta range for data collection

$0.42 \times 0.07 \times 0.07 \mathrm{~mm}$

3.48 to $27.52 \mathrm{deg}$.

$-18<=\mathrm{h}<=18, \quad-7<=\mathrm{k}<=5, \quad-39<=1<=38$

$23470 / 5061[\mathrm{R}($ int $)=0.0751]$

Reflections collected / unique

Completeness to theta $=27.52$

$97.1 \%$

Gaussian

Absorption correction

Max. and min. transmission

Refinement method

Data / restraints / parameters

0.9628 and 0.9042

Full-matrix least-squares on $\mathrm{F}^{\wedge} 2$

5061 / 0 / 339

Goodness-of-fit on $\mathrm{F}^{\wedge} 2$

1.015

Final $R$ indices [I $>2$ sigma(I)]

$R$ indices (all data)

$\mathrm{R} 1=0.0556, w R 2=0.0925[2958 \mathrm{Fo}]$

Largest diff. peak and hole

$R 1=0.1213, w R 2=0.1106$

0.301 and -0.387 e. $A^{\wedge}-3$

Table S7. Atomic coordinates and equivalent isotropic displacement parameters for 2.H2O. $\mathrm{U}(\mathrm{eq})$ is defined as one third of the trace of the orthogonalized Uij tensor.

\begin{tabular}{ccccc}
\hline & $x$ & $y$ & $z$ & $U(e q)$ \\
\hline$C(01)$ & $0.6931(2)$ & $0.0984(6)$ & $0.93550(10)$ & $0.0343(7)$ \\
$C(02)$ & $0.7336(2)$ & $-0.0683(6)$ & $0.96576(10)$ & $0.0361(7)$
\end{tabular}




\begin{tabular}{|c|c|c|c|c|}
\hline$C(03)$ & $0.8494(2)$ & $-0.3337(6)$ & $1.02254(10)$ & $0.0404(8)$ \\
\hline$c(04)$ & $0.7605(2)$ & $-0.4165(6)$ & $1.02508(10)$ & $0.0376(7)$ \\
\hline$C(05)$ & $0.9704(4)$ & $-0.2180(16)$ & $1.09980(19)$ & $0.0900(18)$ \\
\hline$c(06)$ & $0.6112(3)$ & $-0.6746(12)$ & $1.0515(2)$ & $0.0714(13)$ \\
\hline$c(07)$ & $0.66319(19)$ & $0.4365(5)$ & $0.87537(9)$ & $0.0293(7)$ \\
\hline$C(08)$ & $0.57148(19)$ & $0.3364(5)$ & $0.87671(9)$ & $0.0315(7)$ \\
\hline$C(09)$ & $0.65144(18)$ & $0.6174(5)$ & $0.84274(9)$ & $0.0283(7)$ \\
\hline$C(10)$ & $0.72207(19)$ & $0.7761(5)$ & $0.82774(9)$ & $0.0288(7)$ \\
\hline$C(11)$ & $0.6963(2)$ & $0.9922(6)$ & $0.80430(10)$ & $0.0321(7)$ \\
\hline$C(12)$ & $0.7670(2)$ & $1.1328(6)$ & $0.79106(10)$ & $0.0381(8)$ \\
\hline$C(13)$ & $0.8851(2)$ & $0.8687(7)$ & $0.81998(12)$ & $0.0495(9)$ \\
\hline$C(14)$ & $0.8205(2)$ & $0.7173(7)$ & $0.83579(12)$ & $0.0407(8)$ \\
\hline$C(15)$ & $0.50402(19)$ & $0.4543(5)$ & $0.84475(9)$ & $0.0303(7)$ \\
\hline$C(16)$ & $0.39978(19)$ & $0.4182(5)$ & $0.83229(9)$ & $0.0319(7)$ \\
\hline$C(17)$ & $0.3408(2)$ & $0.5792(7)$ & $0.80435(12)$ & $0.0484(9)$ \\
\hline$C(18)$ & $0.2424(2)$ & $0.5365(8)$ & $0.79365(13)$ & $0.0556(10)$ \\
\hline$C(19)$ & $0.2544(2)$ & $0.1965(8)$ & $0.83570(14)$ & $0.0583(11)$ \\
\hline$C(20)$ & $0.3535(2)$ & $0.2235(7)$ & $0.84766(12)$ & $0.0514(10)$ \\
\hline$N(01)$ & $0.55398(16)$ & $0.6263(5)$ & $0.82507(8)$ & $0.0318(6)$ \\
\hline$N(02)$ & $0.86112(19)$ & $1.0771(5)$ & $0.79800(9)$ & $0.0451(7)$ \\
\hline$N(03)$ & $0.19796(18)$ & $0.3495(6)$ & $0.80890(10)$ & $0.0511(8)$ \\
\hline$S(01)$ & $0.76238(5)$ & $0.31929(15)$ & $0.91269(3)$ & $0.0383(2)$ \\
\hline$S(02)$ & $0.56605(5)$ & $0.10736(16)$ & $0.91642(3)$ & $0.0427(2)$ \\
\hline$S(03)$ & $0.85882(5)$ & $-0.09422(17)$ & $0.98483(3)$ & $0.0477(2)$ \\
\hline$S(04)$ & $0.66287(5)$ & $-0.27811(16)$ & $0.98981(3)$ & $0.0467(2)$ \\
\hline$S(05)$ & $0.95711(6)$ & $-0.44194(18)$ & $1.05555(3)$ & $0.0557(3)$ \\
\hline$S(06)$ & $0.73969(7)$ & $-0.64825(19)$ & $1.06221(3)$ & $0.0623(3)$ \\
\hline $0(01)$ & $0.4964(2)$ & $0.8365(5)$ & $0.73903(8)$ & $0.0521(7)$ \\
\hline$H(01)$ & $0.534(2)$ & $0.701(5)$ & $0.7996(10)$ & $0.036(9)$ \\
\hline$H(01 A)$ & $0.537(4)$ & $0.764(9)$ & $0.7261(16)$ & $0.11(2)$ \\
\hline$H(01 B)$ & $0.448(3)$ & $0.825(8)$ & $0.7233(14)$ & $0.083(16)$ \\
\hline$H(05 A)$ & $0.925(5)$ & $-0.306(11)$ & $1.113(2)$ & $0.11(3)$ \\
\hline$H(05 B)$ & $0.951(5)$ & $-0.070(14)$ & $1.093(2)$ & $0.14(4)$ \\
\hline$H(05 C)$ & $1.033(4)$ & $-0.248(9)$ & $1.1181(16)$ & $0.116(17)$ \\
\hline$H(06 A)$ & $0.587(4)$ & $-0.531(11)$ & $1.0596(18)$ & $0.13(2)$ \\
\hline$H(06 B)$ & $0.592(3)$ & $-0.722(8)$ & $1.0233(16)$ & $0.090(17)$ \\
\hline$H(06 C)$ & $0.599(4)$ & $-0.806(11)$ & $1.072(2)$ & $0.11(2)$ \\
\hline$H(11)$ & $0.633(2)$ & $1.043(5)$ & $0.7987(9)$ & $0.032(8)$ \\
\hline$H(12)$ & $0.755(2)$ & $1.279(6)$ & $0.7763(10)$ & $0.040(9)$ \\
\hline$H(13)$ & $0.951(2)$ & $0.825(5)$ & $0.8244(9)$ & $0.046(9)$ \\
\hline$H(14)$ & $0.8405(19)$ & $0.578(5)$ & $0.8478(9)$ & $0.028(8)$ \\
\hline $\mathrm{H}(17)$ & $0.363(2)$ & $0.708(6)$ & $0.7918(10)$ & $0.042(9)$ \\
\hline$H(18)$ & $0.203(3)$ & $0.654(7)$ & $0.7748(12)$ & $0.077(12)$ \\
\hline$H(19)$ & $0.227(3)$ & $0.063(7)$ & $0.8461(12)$ & $0.075(13)$ \\
\hline$H(20)$ & $0.383(2)$ & $0.109(5)$ & $0.8666(10)$ & $0.036(8)$ \\
\hline
\end{tabular}

Table S8. Bond lengths [A] for 2.H2O.

\begin{tabular}{ll}
\hline$C(01)-C(02)$ & $1.340(4)$ \\
$C(01)-S(01)$ & $1.763(3)$ \\
$C(01)-S(02)$ & $1.770(3)$ \\
$C(02)-S(03)$ & $1.750(3)$ \\
$C(02)-S(04)$ & $1.756(3)$ \\
$C(03)-C(04)$ & $1.340(4)$ \\
$C(03)-S(03)$ & $1.753(3)$ \\
$C(03)-S(05)$ & $1.753(3)$ \\
$C(04)-S(04)$ & $1.746(3)$ \\
$C(04)-S(06)$ & $1.749(3)$
\end{tabular}




\begin{tabular}{ll}
$\mathrm{C}(05)-\mathrm{S}(05)$ & $1.795(6)$ \\
$\mathrm{C}(05)-\mathrm{H}(05 \mathrm{~A})$ & $0.94(6)$ \\
$\mathrm{C}(05)-\mathrm{H}(05 \mathrm{~B})$ & $0.87(7)$ \\
$\mathrm{C}(05)-\mathrm{H}(05 \mathrm{C})$ & $0.96(5)$ \\
$\mathrm{C}(06)-\mathrm{S}(06)$ & $1.776(5)$ \\
$\mathrm{C}(06)-\mathrm{H}(06 \mathrm{~A})$ & $0.90(6)$ \\
$\mathrm{C}(06)-\mathrm{H}(06 \mathrm{~B})$ & $0.88(4)$ \\
$\mathrm{C}(06)-\mathrm{H}(06 \mathrm{C})$ & $0.98(6)$ \\
$\mathrm{C}(07)-\mathrm{C}(09)$ & $1.383(4)$ \\
$\mathrm{C}(07)-\mathrm{C}(08)$ & $1.403(4)$ \\
$\mathrm{C}(07)-\mathrm{S}(01)$ & $1.744(3)$ \\
$\mathrm{C}(08)-\mathrm{C}(15)$ & $1.381(4)$ \\
$\mathrm{C}(08)-\mathrm{S}(02)$ & $1.743(3)$ \\
$\mathrm{C}(09)-\mathrm{N}(01)$ & $1.374(3)$ \\
$\mathrm{C}(09)-\mathrm{C}(10)$ & $1.448(4)$ \\
$\mathrm{C}(10)-\mathrm{C}(11)$ & $1.387(4)$ \\
$\mathrm{C}(10)-\mathrm{C}(14)$ & $1.394(4)$ \\
$\mathrm{C}(11)-\mathrm{C}(12)$ & $1.368(4)$ \\
$\mathrm{C}(11)-\mathrm{H}(11)$ & $0.91(3)$ \\
$\mathrm{C}(12)-\mathrm{N}(02)$ & $1.333(4)$ \\
$\mathrm{C}(12)-\mathrm{H}(12)$ & $0.91(3)$ \\
$\mathrm{C}(13)-\mathrm{N}(02)$ & $1.328(4)$ \\
$\mathrm{C}(13)-\mathrm{C}(14)$ & $1.373(5)$ \\
$\mathrm{C}(13)-\mathrm{H}(13)$ & $0.94(3)$ \\
$\mathrm{C}(14)-\mathrm{H}(14)$ & $0.86(3)$ \\
$\mathrm{C}(15)-\mathrm{N}(01)$ & $1.369(4)$ \\
$\mathrm{C}(15)-\mathrm{C}(16)$ & $1.454(4)$ \\
$\mathrm{C}(16)-\mathrm{C}(20)$ & $1.368(4)$ \\
$\mathrm{C}(16)-\mathrm{C}(17)$ & $1.383(4)$ \\
$\mathrm{C}(17)-\mathrm{C}(18)$ & $1.379(5)$ \\
$\mathrm{C}(17)-\mathrm{H}(17)$ & $0.89(3)$ \\
$\mathrm{C}(18)-\mathrm{N}(03)$ & $1.320(4)$ \\
$\mathrm{C}(18)-\mathrm{H}(18)$ & $0.96(4)$ \\
$\mathrm{C}(19)-\mathrm{N}(03)$ & $1.320(4)$ \\
$\mathrm{C}(19)-\mathrm{C}(20)$ & $1.378(4)$ \\
$\mathrm{C}(19)-\mathrm{H}(19)$ & $0.90(4)$ \\
$\mathrm{C}(20)-\mathrm{H}(20)$ & $0.89(3)$ \\
$\mathrm{N}(01)-\mathrm{H}(01)$ & $0.87(3)$ \\
$\mathrm{O}(01)-\mathrm{H}(01 \mathrm{~A})$ & $0.84(5)$ \\
$\mathrm{O}(01)-\mathrm{H}(01 \mathrm{~B})$ & $0.75(4)$ \\
& \\
\hline
\end{tabular}

Table 59. Bond angles [deg] for 2.H2O.

\begin{tabular}{|ll|ll|}
\hline & & & \\
$C(02)-C(01)-S(01)$ & $122.3(2)$ & $N(02)-C(12)-C(11)$ & $125.1(3)$ \\
$C(02)-C(01)-S(02)$ & $121.2(2)$ & $N(02)-C(12)-H(12)$ & $111.5(19)$ \\
$S(01)-C(01)-S(02)$ & $116.54(16)$ & $C(11)-C(12)-H(12)$ & $123.4(19)$ \\
$C(01)-C(02)-S(03)$ & $123.7(2)$ & $N(02)-C(13)-C(14)$ & $124.4(3)$ \\
$C(01)-C(02)-S(04)$ & $121.5(2)$ & $N(02)-C(13)-H(13)$ & $116.0(19)$ \\
$S(03)-C(02)-S(04)$ & $114.78(17)$ & $C(14)-C(13)-H(13)$ & $119.6(19)$ \\
$C(04)-C(03)-S(03)$ & $118.0(2)$ & $C(13)-C(14)-C(10)$ & $119.7(3)$ \\
$C(04)-C(03)-S(05)$ & $124.6(2)$ & $C(13)-C(14)-H(14)$ & $119.2(19)$ \\
$S(03)-C(03)-S(05)$ & $117.46(18)$ & $C(10)-C(14)-H(14)$ & $120.7(19)$ \\
$C(03)-C(04)-S(04)$ & $116.9(2)$ & $N(01)-C(15)-C(08)$ & $106.4(2)$ \\
$C(03)-C(04)-S(06)$ & $123.0(2)$ & $N(01)-C(15)-C(16)$ & $123.3(2)$ \\
$S(04)-C(04)-S(06)$ & $120.00(17)$ & $C(08)-C(15)-C(16)$ & $130.2(3)$ \\
$S(05)-C(05)-H(05 A)$ & $89(4)$ & $C(20)-C(16)-C(17)$ & $115.6(3)$ \\
$S(05)-C(05)-H(05 B)$ & $117(5)$ & $C(20)-C(16)-C(15)$ & $122.2(3)$ \\
\hline
\end{tabular}




\begin{tabular}{|ll|ll|}
\hline$H(05 A)-C(05)-H(05 B)$ & $112(6)$ & $C(17)-C(16)-C(15)$ & $122.2(3)$ \\
$\mathrm{S}(05)-\mathrm{C}(05)-\mathrm{H}(05 \mathrm{C})$ & $106(3)$ & $\mathrm{C}(18)-\mathrm{C}(17)-\mathrm{C}(16)$ & $119.8(4)$ \\
$\mathrm{H}(05 \mathrm{~A})-\mathrm{C}(05)-\mathrm{H}(05 \mathrm{C})$ & $107(5)$ & $\mathrm{C}(18)-\mathrm{C}(17)-\mathrm{H}(17)$ & $117(2)$ \\
$\mathrm{H}(05 \mathrm{~B})-\mathrm{C}(05)-\mathrm{H}(05 \mathrm{C})$ & $121(6)$ & $\mathrm{C}(16)-\mathrm{C}(17)-\mathrm{H}(17)$ & $123(2)$ \\
$\mathrm{S}(06)-\mathrm{C}(06)-\mathrm{H}(06 \mathrm{~A})$ & $107(4)$ & $\mathrm{N}(03)-\mathrm{C}(18)-\mathrm{C}(17)$ & $124.3(4)$ \\
$\mathrm{S}(06)-\mathrm{C}(06)-\mathrm{H}(06 \mathrm{~B})$ & $108(3)$ & $\mathrm{N}(03)-\mathrm{C}(18)-\mathrm{H}(18)$ & $118(2)$ \\
$\mathrm{H}(06 \mathrm{~A})-\mathrm{C}(06)-\mathrm{H}(06 \mathrm{~B})$ & $117(5)$ & $\mathrm{C}(17)-\mathrm{C}(18)-\mathrm{H}(18)$ & $118(2)$ \\
$\mathrm{S}(06)-\mathrm{C}(06)-\mathrm{C}(06 \mathrm{C})$ & $103(3)-\mathrm{C}(20)$ & $123.8(4)$ \\
$\mathrm{H}(06 \mathrm{~A})-\mathrm{C}(06)-\mathrm{H}(06 \mathrm{C})$ & $110(5)$ & $\mathrm{N}(03)-\mathrm{C}(19)-\mathrm{H}(19)$ & $118(2)$ \\
$\mathrm{H}(06 \mathrm{~B})-\mathrm{C}(06)-\mathrm{H}(06 \mathrm{C})$ & $110(4)$ & $\mathrm{C}(20)-\mathrm{C}(19)-\mathrm{H}(19)$ & $118(2)$ \\
$\mathrm{C}(09)-\mathrm{C}(07)-\mathrm{C}(08)$ & $107.8(2)$ & $\mathrm{C}(16)-\mathrm{C}(20)-\mathrm{C}(19)$ & $120.7(3)$ \\
$\mathrm{C}(09)-\mathrm{C}(07)-\mathrm{S}(01)$ & $134.4(2)$ & $\mathrm{C}(16)-\mathrm{C}(20)-\mathrm{H}(20)$ & $124.7(19)$ \\
$\mathrm{C}(08)-\mathrm{C}(07)-\mathrm{S}(01)$ & $117.8(2)$ & $\mathrm{C}(19)-\mathrm{C}(20)-\mathrm{H}(20)$ & $114.5(19)$ \\
$\mathrm{C}(15)-\mathrm{C}(08)-\mathrm{C}(07)$ & $108.3(2)$ & $\mathrm{C}(15)-\mathrm{N}(01)-\mathrm{C}(09)$ & $111.0(2)$ \\
$\mathrm{C}(15)-\mathrm{C}(08)-\mathrm{S}(02)$ & $134.8(2)$ & $\mathrm{C}(15)-\mathrm{N}(01)-\mathrm{H}(01)$ & $126(2)$ \\
$\mathrm{C}(07)-\mathrm{C}(08)-\mathrm{S}(02)$ & $116.9(2)$ & $\mathrm{C}(09)-\mathrm{N}(01)-\mathrm{H}(01)$ & $120.2(19)$ \\
$\mathrm{N}(01)-\mathrm{C}(09)-\mathrm{C}(07)$ & $106.5(2)$ & $\mathrm{C}(13)-\mathrm{N}(02)-\mathrm{C}(12)$ & $115.3(3)$ \\
$\mathrm{N}(01)-\mathrm{C}(09)-\mathrm{C}(10)$ & $123.0(2)$ & $\mathrm{C}(18)-\mathrm{N}(03)-\mathrm{C}(19)$ & $115.7(3)$ \\
$\mathrm{C}(07)-\mathrm{C}(09)-\mathrm{C}(10)$ & $130.5(2)$ & $\mathrm{C}(07)-\mathrm{S}(01)-\mathrm{C}(01)$ & $94.12(14)$ \\
$\mathrm{C}(11)-\mathrm{C}(10)-\mathrm{C}(14)$ & $116.2(3)$ & $\mathrm{C}(08)-\mathrm{S}(02)-\mathrm{C}(01)$ & $94.40(14)$ \\
$\mathrm{C}(11)-\mathrm{C}(10)-\mathrm{C}(09)$ & $122.5(3)$ & $\mathrm{C}(02)-\mathrm{S}(03)-\mathrm{C}(03)$ & $94.85(14)$ \\
$\mathrm{C}(14)-\mathrm{C}(10)-\mathrm{C}(09)$ & $121.3(3)$ & $\mathrm{C}(04)-\mathrm{S}(04)-\mathrm{C}(02)$ & $95.45(15)$ \\
$\mathrm{C}(12)-\mathrm{C}(11)-\mathrm{C}(10)$ & $119.3(3)$ & $\mathrm{C}(03)-\mathrm{S}(05)-\mathrm{C}(05)$ & $99.1(2)$ \\
$\mathrm{C}(12)-\mathrm{C}(11)-\mathrm{H}(11)$ & $120.1(18)$ & $\mathrm{C}(04)-\mathrm{S}(06)-\mathrm{C}(06)$ & $102.9(2)$ \\
$\mathrm{C}(10)-\mathrm{C}(11)-\mathrm{H}(11)$ & $120.5(18)$ & $\mathrm{H}(01 \mathrm{~A})-0(01)-\mathrm{H}(01 \mathrm{~B})$ & $105(4)$ \\
& & & \\
\hline
\end{tabular}

Table S10. Anisotropic displacement parameters for 2.H2O.

The anisotropic displacement factor exponent takes the form:

$-2 \mathrm{pi}^{\wedge} 2\left[\mathrm{~h}^{\wedge} 2 \mathrm{a}^{* \wedge} 2 \mathrm{U11}+\ldots+2 \mathrm{~h} \mathrm{k} \mathrm{a}^{*} \mathrm{~b}^{*} \mathrm{U} 12\right]$

$\begin{array}{llllll}\text { U11 } & \text { U22 } & \text { U33 } & \text { U23 } & \text { U13 } & \text { U12 }\end{array}$

\begin{tabular}{|c|c|c|c|c|c|c|}
\hline$C(\overline{01})$ & $0.0249(15)$ & $0.0417(19)$ & $0.0350(16)$ & $0.0058(15)$ & $0.0022(12)$ & $-0.0026(14)$ \\
\hline$C(02)$ & $0.0299(16)$ & $0.0375(19)$ & $0.0392(17)$ & $0.0036(15)$ & $0.0020(13)$ & (14) \\
\hline$c(03)$ & $0.0353(18)$ & $0.041(2)$ & $0.0402(18)$ & $0.0047(15)$ & $-0.0057(14)$ & $0.0063(15)$ \\
\hline$c(04)$ & $0.0355(18)$ & $0.0378(19)$ & $0.0373(17)$ & $0.0062(15)$ & $0.0012(13)$ & $0.0016(15)$ \\
\hline$c(05)$ & $0.058(3)$ & $0.134(6)$ & $0.068(3)$ & $-0.025(3)$ & $-0.016(3)$ & $0.019(4)$ \\
\hline$c(06)$ & $0.065(3)$ & $0.078(4)$ & $0.071(3)$ & $0.021(3)$ & $0.015(3)$ & $-0.018(3)$ \\
\hline$c(07)$ & 0.020 & $0.0324(17)$ & $0.0336(16)$ & $0.0004(14)$ & $0.0022(12)$ & $-0.0018(13)$ \\
\hline$c(08)$ & $0.0258(16)$ & $0.0328(18)$ & $0.0353(16)$ & $0.0017(14)$ & $0.0042(12)$ & $-0.0024(13)$ \\
\hline$C(09)$ & $0.0221(15)$ & $0.0315(17)$ & $0.0308(15)$ & $0.0013(13)$ & $0.0032(12)$ & 13) \\
\hline$C(10)$ & $0.0268(15)$ & $0.0322(18)$ & $0.0269(15)$ & $-0.0051(13)$ & $0.0035(12)$ & $-0.0043(13)$ \\
\hline$C(11)$ & & (19) & & & & \\
\hline$C(12)$ & $0.044(2)$ & $0.035(2)$ & $0.0342(17)$ & $0.0001(16)$ & $0.0057(14)$ & (17) \\
\hline$C(13)$ & 0.024 & $0.055(3)$ & $0.069(2)$ & $-0.002(2)$ & $0.0072(16)$ & -0.00 \\
\hline$C(14)$ & $0.0287(18)$ & $0.034(2)$ & $0.058(2)$ & $0.0081(17)$ & $0.0055(15)$ & $9(16)$ \\
\hline$C(15)$ & $0.0236(15)$ & $0.0345(18)$ & $0.0316(16)$ & $0.0013(14)$ & $0.0016(12)$ & $-0.0023(13)$ \\
\hline$C(16)$ & $0.0227(15)$ & $0.0388(19)$ & $0.0335(16)$ & $0.0012(14)$ & $0.0033(12)$ & $-0.0022(14)$ \\
\hline$C(17)$ & $0.0281(18)$ & $0.059(3)$ & $0.056(2)$ & $0.017(2)$ & $0.0006(15)$ & $-0.0045(17)$ \\
\hline$C(18)$ & $0.030(2)$ & $0.072(3)$ & $0.060(2)$ & $0.012(2)$ & $-0.0063(17)$ & $0.003(2)$ \\
\hline$C(19)$ & $0.031(2)$ & $0.065(3)$ & $0.077(3)$ & $0.014(2)$ & $0.0022(18)$ & $-0.014(2)$ \\
\hline$C(20)$ & $0.0322(19)$ & $0.055(2)$ & $0.063(2)$ & $0.022(2)$ & $6(17)$ & (18) \\
\hline$N(01)$ & $0.0229(13)$ & $0.0391(16)$ & $0.0316(14)$ & $0.0078(12)$ & $0.0002(11)$ & $-0.0016(12)$ \\
\hline$N(02)$ & $0.0398(17)$ & $0.0444(18)$ & $0.0524(17)$ & $0.0003(15)$ & $0.0121(13)$ & $-0.0131(14)$ \\
\hline$N(03)$ & $0.0241(14)$ & $0.070(2)$ & $0.0569(18)$ & $-0.0019(16)$ & $0.0019(13)$ & $-0.0071(15)$ \\
\hline $5(01)$ & $0.0231(4)$ & $0.0465(5)$ & $0.0422(4)$ & $0.0116(4)$ & $-0.0024(3)$ & $-0.0038(3)$ \\
\hline$(02)$ & $0.0248(4)$ & $0.0508(6)$ & $0.0501(5)$ & $0.0196(4)$ & $0.0003(3)$ & $-0.0051(4)$ \\
\hline
\end{tabular}


$\mathrm{S}(03)$

$\mathrm{S}(04)$

$\mathrm{S}(05)$

$\mathrm{S}(06)$

$\mathrm{O}(01)$
$0.0256(4) \quad 0.0565(6)$
$0.0283(4) \quad 0.0521(6)$
$0.0399(5) \quad 0.0659(7)$
$0.0575(6) \quad 0.0671(7)$
$0.0292(14) 0.086(2)$

$0.0580(5) \quad 0.0187(4)-0.0003(4)-0.0015(4)$

$\begin{array}{llll}0.0575(5) & 0.0209(4) & 0.0022(4) & -0.0017(4)\end{array}$

$\begin{array}{llll}0.0546(6) & 0.0086(5) & -0.0085(4) & 0.0137(5)\end{array}$

$0.0593(6) \quad 0.0299(5) \quad 0.0031(5)-0.0039(5)$

$0.0380(14)-0.0015(13)-0.0018(11) 0.0127(14)$

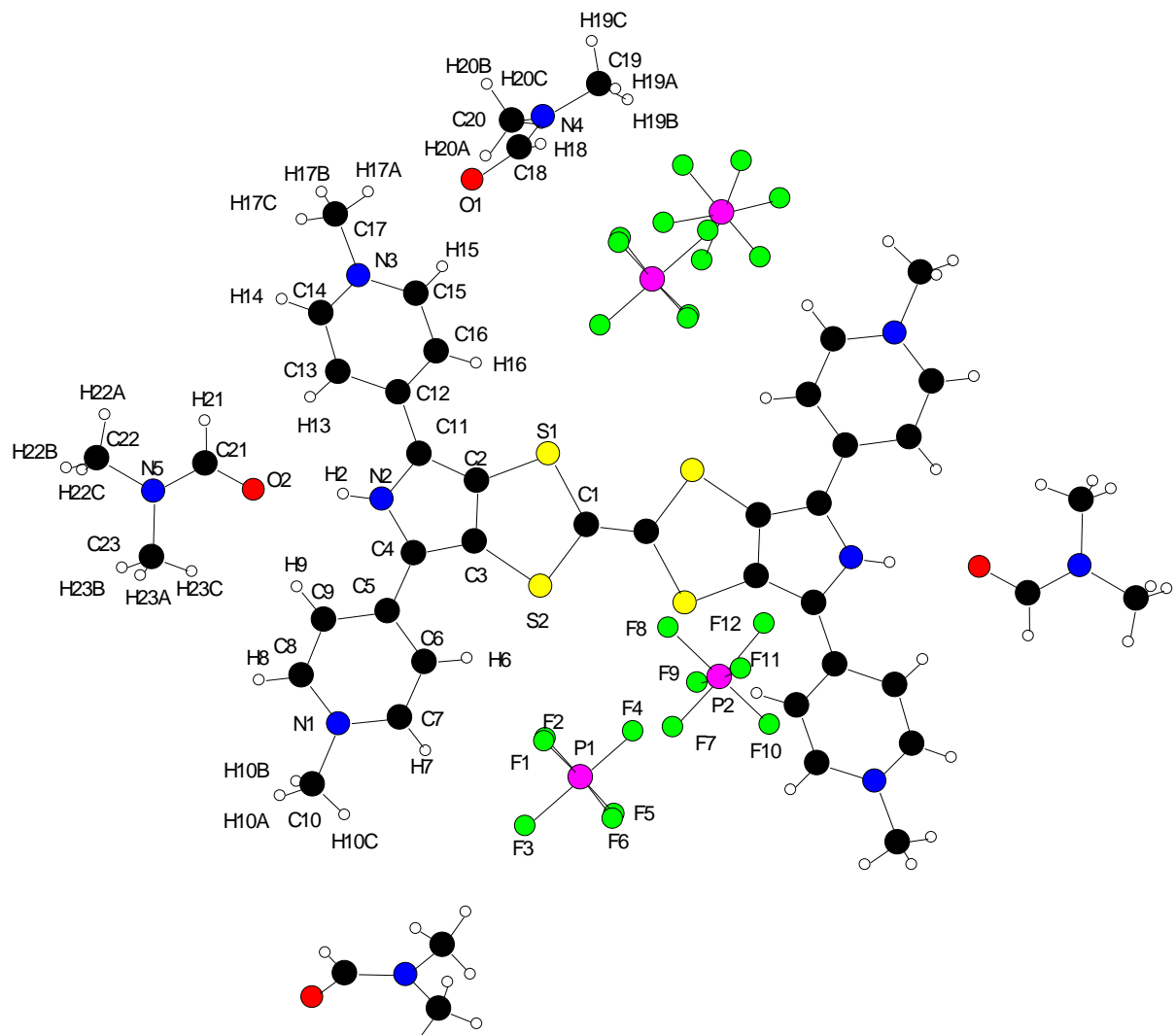

Figure S30. X-ray structure of 5

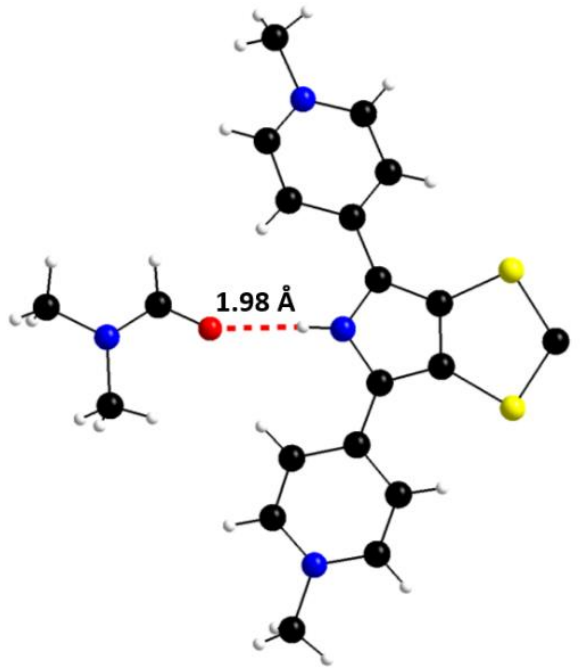

Figure S31. Molecular structure of 5 (only one half of the molecule is shown); H-Bond interaction between one DMF solvent molecule and the NH group (pyrrole) 


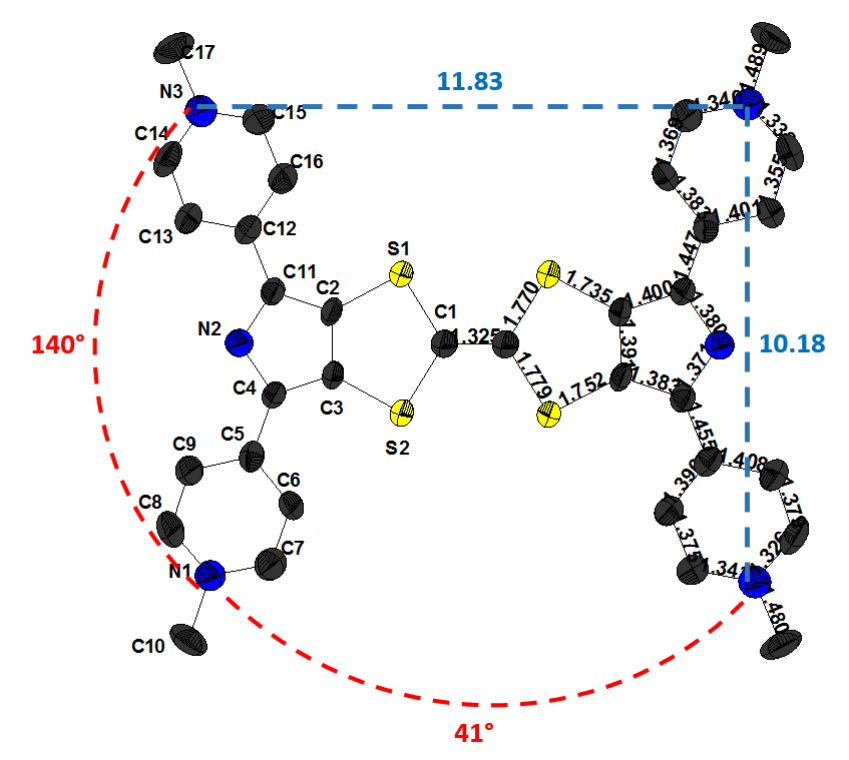

Figure S32. $\mathrm{N}_{\text {pyridine }} \cdots \mathrm{N}_{\text {pyridine }}$ distances within one molecule of 5 and the associated coordination angles between two adjacent BPTTF-Pyr axes.

Table S11. Crystal data and structure refinement for $\mathbf{5}$

Empirical formula

Formula weight

Temperature

Wavelength

Crystal system, space group

Unit cell dimensions

Volume

Z, Calculated density Absorption coefficient

$\mathrm{F}(000)$

Crystal size

Theta range for data collection

Limiting indices

Reflections collected / unique
C46 H58 F24 N10 O4 P4 S4

1523.14

200(2) K

$0.71073 \mathrm{~A}$

Monoclinic, P 1 21/n 1

$\mathrm{a}=6.3094(5)$ A alpha $=90 \mathrm{deg}$.

$\mathrm{b}=19.889(1)$ A beta $=96.084(8) \mathrm{deg}$.

$\mathrm{c}=25.432(1) \mathrm{A}$ gamma $=90 \mathrm{deg}$.

3173.4(3) $\mathrm{A}^{\wedge} 3$

$2,1.594 \mathrm{Mg} / \mathrm{m}^{\wedge} 3$

$0.372 \mathrm{~mm}^{\wedge}-1$

1552

$0.30 \times 0.07 \times 0.04 \mathrm{~mm}$

2.61 to $25.99 \mathrm{deg}$.

$-7<=\mathrm{h}<=7,-22<=\mathrm{k}<=24,-30<=\mathrm{l}<=30$

$24250 / 6000[\mathrm{R}(\mathrm{int})=0.0972]$ 
Completeness to theta $=25.00$

Absorption correction

Max. and min. transmission

Refinement method

Data / restraints / parameters

Goodness-of-fit on $\mathrm{F}^{\wedge} 2$

Final R indices [I $>2 \operatorname{sigma}(\mathrm{I})]$

$\mathrm{R}$ indices (all data)

Largest diff. peak and hole
$98.2 \%$

Semi-empirical from equivalents

0.985 and 0.906

Full-matrix least-squares on $\mathrm{F}^{\wedge} 2$

$6000 / 0 / 421$

1.025

$\mathrm{R} 1=0.0882, \mathrm{wR} 2=0.1745$ [2889 Fo]

$\mathrm{R} 1=0.2037, \mathrm{wR} 2=0.2292$

0.830 and -0.534 e. $\mathrm{A}^{\wedge}-3$

Table S12. Atomic coordinates and equivalent isotropic displacement parameters for $\mathbf{5}$.

$U(e q)$ is defined as one third of the trace of the orthogonalized Uij tensor.

\begin{tabular}{|c|c|c|c|c|}
\hline & $\mathrm{x}$ & $\mathrm{Y}$ & $z$ & $\mathrm{U}(\mathrm{eq})$ \\
\hline$C(1)$ & $0.4225(10)$ & $0.9906(3)$ & $0.0141(3)$ & $0.0316(16)$ \\
\hline$C(2)$ & $0.1272(10)$ & $0.9239(3)$ & $0.0540(3)$ & $0.0281(15)$ \\
\hline$C(3)$ & $0.1160(10)$ & 0.9895 (3) & $0.0723(2)$ & $0.0280(15)$ \\
\hline$C(4)$ & $-0.0504(10)$ & $0.9923(3)$ & $0.1044(3)$ & $0.0293(15)$ \\
\hline$C(5)$ & $-0.1119(10)$ & $1.0483(3)$ & $0.1358(3)$ & $0.0306(15)$ \\
\hline$C(6)$ & $-0.0265(11)$ & $1.1118(3)$ & $0.1307(3)$ & $0.0366(18)$ \\
\hline$C(7)$ & $-0.0783(11)$ & $1.1645(4)$ & $0.1616(3)$ & $0.0412(18)$ \\
\hline$C(8)$ & $-0.3046(12)$ & $1.0946(4)$ & $0.2033(3)$ & $0.047(2)$ \\
\hline$C(9)$ & $-0.2568(12)$ & $1.0414(4)$ & $0.1737(3)$ & $0.0438(19)$ \\
\hline$C(10)$ & $-0.2711(14)$ & $1.2132(4)$ & $0.2307(3)$ & $0.053(2)$ \\
\hline C (11) & $-0.0300(10)$ & $0.8856(3)$ & $0.0734(3)$ & $0.0285(15)$ \\
\hline C (12) & $-0.0787(11)$ & $0.8144(3)$ & $0.0667(3)$ & $0.0328(16)$ \\
\hline$C(13)$ & $-0.2553(12)$ & $0.7847(4)$ & $0.0871(3)$ & $0.0404(18)$ \\
\hline$C(14)$ & $-0.2901(12)$ & $0.7170(4)$ & $0.0800(3)$ & 0.0445 (19) \\
\hline$C(15)$ & $0.0095(12)$ & $0.7041(4)$ & $0.0357(3)$ & $0.0409(18)$ \\
\hline$C(16)$ & $0.0525(11)$ & $0.7717(3)$ & $0.0410(3)$ & $0.0380(18)$ \\
\hline$C(17)$ & $-0.2034(14)$ & $0.6046(4)$ & $0.0516(4)$ & $0.057(2)$ \\
\hline$C(18)$ & $0.3423(18)$ & $0.5551(4)$ & $-0.0484(5)$ & $0.073(3)$ \\
\hline C (19) & $0.342(2)$ & $0.5406(6)$ & $-0.1419(4)$ & $0.101(4)$ \\
\hline$C(20)$ & $0.0259(18)$ & $0.5798(7)$ & $-0.1027(5)$ & $0.117(5)$ \\
\hline$C(21)$ & $0.3695(14)$ & $0.8632(5)$ & $0.1756(3)$ & $0.058(2)$ \\
\hline$C(22)$ & $0.0639(15)$ & $0.8416(6)$ & $0.2238(4)$ & $0.085(3)$ \\
\hline C (23) & $0.2712(18)$ & $0.9461(6)$ & $0.2365(5)$ & $0.097(4)$ \\
\hline $\mathrm{F}(1)$ & $0.4390(9)$ & 0.1911 (3) & $0.1207(2)$ & $0.092(2)$ \\
\hline$F(2)$ & $0.1652(9)$ & $0.2396(3)$ & $0.0722(2)$ & $0.102(2)$ \\
\hline$F(3)$ & $0.3230(11)$ & $0.2908(4)$ & $0.1441(2)$ & $0.110(2)$ \\
\hline $\mathrm{F}(4)$ & $0.4775(13)$ & $0.2332(4)$ & $0.0409(3)$ & $0.128(3)$ \\
\hline$F(5)$ & $0.6373(8)$ & $0.2834(3)$ & $0.1131(3)$ & $0.098(2)$ \\
\hline$F(6)$ & $0.3594(9)$ & $0.3311(3)$ & $0.0629(2)$ & $0.0848(17)$ \\
\hline$F(7)$ & $0.2623(10)$ & $0.6071(4)$ & $0.1234(2)$ & $0.124(3)$ \\
\hline $\mathrm{F}(8)$ & $0.3797(17)$ & $0.6953(4)$ & $0.1686(4)$ & $0.182(4)$ \\
\hline$F(9)$ & $0.4808(11)$ & $0.5979(4)$ & $0.1968(3)$ & $0.142(3)$ \\
\hline $\mathrm{F}(10)$ & $0.1323(17)$ & $0.5690(5)$ & $0.1932(4)$ & $0.182(4)$ \\
\hline $\mathrm{F}(11)$ & $0.0375(10)$ & $0.6656(5)$ & $0.1666(2)$ & $0.138(3)$ \\
\hline $\mathrm{F}(12)$ & $0.2559(9)$ & $0.6579(4)$ & $0.2389(2)$ & $0.118(3)$ \\
\hline $\mathrm{N}(1)$ & $-0.2148(9)$ & $1.1550(3)$ & $0.1980(2)$ & $0.0369(14)$ \\
\hline$N(2)$ & $-0.1349(8)$ & $0.9283(3)$ & $0.1042(2)$ & $0.0318(14)$ \\
\hline$N(3)$ & $-0.1604(10)$ & $0.6777(3)$ & $0.0558(2)$ & $0.0393(15)$ \\
\hline$N(4)$ & $0.2393(12)$ & $0.5569(4)$ & $-0.0970(3)$ & $0.062(2)$ \\
\hline$N(5)$ & $0.2416(10)$ & $0.8819(3)$ & $0.2107(3)$ & $0.0485(17)$ \\
\hline$O(1)$ & $0.2727(11)$ & $0.5708(4)$ & $-0.0064(3)$ & $0.083(2)$ \\
\hline$O(2)$ & $0.5240(8)$ & $0.8955(3)$ & $0.1639(2)$ & $0.0591(16)$ \\
\hline
\end{tabular}




\begin{tabular}{|c|c|c|c|c|}
\hline $\mathrm{P}(1)$ & $0.4037(3)$ & $0.26081(10)$ & $0.09117(9)$ & $0.0471(6)$ \\
\hline$P(2)$ & $0.2579(3)$ & $0.63028(11)$ & $0.18152(8)$ & $0.0443(5)$ \\
\hline$S(1)$ & $0.3242(3)$ & $0.90662(9)$ & $0.01227(7)$ & $0.0370(5)$ \\
\hline$S(2)$ & $0.3013(3)$ & $1.04779(9)$ & $0.05485(7)$ & $0.0363(5)$ \\
\hline $\mathrm{H}(2)$ & -0.2398 & 0.9166 & 0.1212 & 0.038 \\
\hline $\mathrm{H}(6)$ & 0.0685 & 1.1189 & 0.1057 & 0.044 \\
\hline $\mathrm{H}(7)$ & -0.0191 & 1.2067 & 0.1573 & 0.049 \\
\hline $\mathrm{H}(8)$ & -0.4021 & 1.0892 & 0.2280 & 0.056 \\
\hline $\mathrm{H}(9)$ & -0.3203 & 0.9999 & 0.1785 & 0.053 \\
\hline $\mathrm{H}(10 \mathrm{~A})$ & -0.2512 & 1.2011 & 0.2674 & 0.079 \\
\hline $\mathrm{H}(10 \mathrm{~B})$ & -0.4173 & 1.2254 & 0.2211 & 0.079 \\
\hline $\mathrm{H}(10 \mathrm{C})$ & -0.1809 & 1.2507 & 0.2246 & 0.079 \\
\hline $\mathrm{H}(13)$ & -0.3471 & 0.8106 & 0.1050 & 0.049 \\
\hline $\mathrm{H}(14)$ & -0.4092 & 0.6979 & 0.0927 & 0.053 \\
\hline $\mathrm{H}(15)$ & 0.0983 & 0.6766 & 0.0181 & 0.049 \\
\hline $\mathrm{H}(16)$ & 0.1714 & 0.7894 & 0.0272 & 0.046 \\
\hline $\mathrm{H}(17 \mathrm{~A})$ & -0.0986 & 0.5836 & 0.0323 & 0.086 \\
\hline $\mathrm{H}(17 \mathrm{~B})$ & -0.3428 & 0.5974 & 0.0334 & 0.086 \\
\hline $\mathrm{H}(17 \mathrm{C})$ & -0.1967 & 0.5855 & 0.0864 & 0.086 \\
\hline $\mathrm{H}(18)$ & 0.4829 & 0.5405 & -0.0458 & 0.088 \\
\hline $\mathrm{H}(19 \mathrm{~A})$ & 0.4892 & 0.5305 & -0.1313 & 0.151 \\
\hline $\mathrm{H}(19 \mathrm{~B})$ & 0.3328 & 0.5781 & -0.1659 & 0.151 \\
\hline $\mathrm{H}(19 \mathrm{C})$ & 0.2743 & 0.5022 & -0.1593 & 0.151 \\
\hline $\mathrm{H}(20 \mathrm{~A})$ & 0.0006 & 0.6065 & -0.0725 & 0.175 \\
\hline $\mathrm{H}(20 \mathrm{~B})$ & -0.0690 & 0.5419 & -0.1053 & 0.175 \\
\hline $\mathrm{H}(20 \mathrm{C})$ & 0.0011 & 0.6066 & -0.1342 & 0.175 \\
\hline $\mathrm{H}(21)$ & 0.3408 & 0.8224 & 0.1584 & 0.070 \\
\hline $\mathrm{H}(22 \mathrm{~A})$ & 0.0482 & 0.8032 & 0.2007 & 0.128 \\
\hline $\mathrm{H}(22 \mathrm{~B})$ & -0.0640 & 0.8680 & 0.2194 & 0.128 \\
\hline $\mathrm{H}(22 \mathrm{C})$ & 0.0897 & 0.8267 & 0.2598 & 0.128 \\
\hline $\mathrm{H}(23 \mathrm{~A})$ & 0.3676 & 0.9413 & 0.2681 & 0.145 \\
\hline $\mathrm{H}(23 \mathrm{~B})$ & 0.1364 & 0.9624 & 0.2455 & 0.145 \\
\hline $\mathrm{H}(23 \mathrm{C})$ & 0.3293 & 0.9774 & 0.2132 & 0.145 \\
\hline
\end{tabular}

Table S12. Bond lengths [A] for 5

\begin{tabular}{ll}
\hline $\mathrm{C}(1)-\mathrm{C}(1) \# 1$ & $1.326(12)$ \\
$\mathrm{C}(1)-\mathrm{S}(2)$ & $1.768(6)$ \\
$\mathrm{C}(1)-\mathrm{S}(1)$ & $1.780(7)$ \\
$\mathrm{C}(2)-\mathrm{C}(11)$ & $1.381(8)$ \\
$\mathrm{C}(2)-\mathrm{C}(3)$ & $1.389(9)$ \\
$\mathrm{C}(2)-\mathrm{S}(1)$ & $1.752(6)$ \\
$\mathrm{C}(3)-\mathrm{C}(4)$ & $1.399(8)$ \\
$\mathrm{C}(3)-\mathrm{S}(2)$ & $1.737(6)$ \\
$\mathrm{C}(4)-\mathrm{N}(2)$ & $1.380(8)$ \\
$\mathrm{C}(4)-\mathrm{C}(5)$ & $1.448(9)$ \\
$\mathrm{C}(5)-\mathrm{C}(6)$ & $1.384(9)$ \\
$\mathrm{C}(5)-\mathrm{C}(9)$ & $1.404(9)$ \\
$\mathrm{C}(6)-\mathrm{C}(7)$ & $1.369(9)$ \\
$\mathrm{C}(6)-\mathrm{H}(6)$ & 0.9300 \\
$\mathrm{C}(7)-\mathrm{N}(1)$ & $1.343(9)$ \\
$\mathrm{C}(7)-\mathrm{H}(7)$ & 0.9300 \\
$\mathrm{C}(8)-\mathrm{N}(1)$ & $1.342(9)$ \\
$\mathrm{C}(8)-\mathrm{C}(9)$ & $1.352(10)$ \\
$\mathrm{C}(8)-\mathrm{H}(8)$ & 0.9300 \\
$\mathrm{C}(9)-\mathrm{H}(9)$ & 0.9300 \\
$\mathrm{C}(10)-\mathrm{N}(1)$ & $1.489(9)$ \\
$\mathrm{C}(10)-\mathrm{H}(10 \mathrm{~A})$ & 0.9600 \\
$\mathrm{C}(10)-\mathrm{H}(10 \mathrm{~B})$ & 0.9600 \\
$\mathrm{C}(10)-\mathrm{H}(10 \mathrm{C})$ & 0.9600 \\
&
\end{tabular}


$\mathrm{C}(11)-\mathrm{N}(2)$

C (11) - C (12)

$C(12)-C(16)$

$C(12)-C(13)$

$C(13)-C(14)$

$\mathrm{C}(13)-\mathrm{H}(13)$

$\mathrm{C}(14)-\mathrm{N}(3)$

C (14) $-\mathrm{H}(14)$

$\mathrm{C}(15)-\mathrm{N}(3)$

C (15) - C (16)

$\mathrm{C}(15)-\mathrm{H}(15)$

$\mathrm{C}(16)-\mathrm{H}(16)$

$\mathrm{C}(17)-\mathrm{N}(3)$

$\mathrm{C}(17)-\mathrm{H}(17 \mathrm{~A})$

$\mathrm{C}(17)-\mathrm{H}(17 \mathrm{~B})$

$\mathrm{C}(17)-\mathrm{H}(17 \mathrm{C})$

$\mathrm{C}(18)-\mathrm{O}(1)$

$\mathrm{C}(18)-\mathrm{N}(4)$

$\mathrm{C}(18)-\mathrm{H}(18)$

$\mathrm{C}(19)-\mathrm{N}(4)$

$\mathrm{C}(19)-\mathrm{H}(19 \mathrm{~A})$

C (19) - H (19B)

$\mathrm{C}(19)-\mathrm{H}(19 \mathrm{C})$

$\mathrm{C}(20)-\mathrm{N}(4)$

$\mathrm{C}(20)-\mathrm{H}(20 \mathrm{~A})$

$\mathrm{C}(20)-\mathrm{H}(20 \mathrm{~B})$

$\mathrm{C}(20)-\mathrm{H}(20 \mathrm{C})$

$\mathrm{C}(21)-\mathrm{O}(2)$

$\mathrm{C}(21)-\mathrm{N}(5)$

$\mathrm{C}(21)-\mathrm{H}(21)$

$\mathrm{C}(22)-\mathrm{N}(5)$

$\mathrm{C}(22)-\mathrm{H}(22 \mathrm{~A})$

$\mathrm{C}(22)-\mathrm{H}(22 \mathrm{~B})$

$\mathrm{C}(22)-\mathrm{H}(22 \mathrm{C})$

$\mathrm{C}(23)-\mathrm{N}(5)$

$\mathrm{C}(23)-\mathrm{H}(23 \mathrm{~A})$

$\mathrm{C}(23)-\mathrm{H}(23 \mathrm{~B})$

$\mathrm{C}(23)-\mathrm{H}(23 \mathrm{C})$

$\mathrm{F}(1)-\mathrm{P}(1)$

$F(2)-P(1)$

$\mathrm{F}(3)-\mathrm{P}(1)$

$\mathrm{F}(4)-\mathrm{P}(1)$

$\mathrm{F}(5)-\mathrm{P}(1)$

$F(6)-P(1)$

$F(7)-P(2)$

$\mathrm{F}(8)-\mathrm{P}(2)$

$F(9)-P(2)$

$\mathrm{F}(10)-\mathrm{P}(2)$

$\mathrm{F}(11)-\mathrm{P}(2)$

$\mathrm{F}(12)-\mathrm{P}(2)$

$\mathrm{N}(2)-\mathrm{H}(2)$
$1.371(8)$

$1.456(9)$

$1.396(9)$

1.407 (9)

1. $373(10)$

0.9300

1.329(9)

0.9300

$1.342(9)$

1.377(9)

0.9300

0.9300

1. 481 (9)

0.9600

0.9600

0.9600

1. 238 (11)

1.335 (12)

0.9300

1.409 (11)

0.9600

0.9600

0.9600

1. 415 (12)

0.9600

0.9600

0.9600

$1.230(9)$

1.318(10)

0.9300

$1.446(10)$

0.9600

0.9600

0.9600

1. $439(12)$

0.9600

0.9600

0.9600

$1.582(5)$

$1.587(6)$

$1.603(6)$

$1.511(7)$

$1.584(6)$

$1.584(5)$

$1.552(6)$

$1.557(8)$

$1.559(6)$

$1.500(8)$

$1.568(6)$

$1.562(6)$

0.8600

Symmetry transformations used to generate equivalent atoms: $\# 1-x+1,-y+2,-z$

Table S13. Bond angles [deg] for $\mathbf{5}$.

\begin{tabular}{ll}
\hline $\mathrm{C}(1) \# 1-\mathrm{C}(1)-\mathrm{S}(2)$ & $121.6(7)$ \\
$\mathrm{C}(1) \# 1-\mathrm{C}(1)-\mathrm{S}(1)$ & $121.8(7)$ \\
$\mathrm{S}(2)-\mathrm{C}(1)-\mathrm{S}(1)$ & $116.6(3)$ \\
$\mathrm{C}(11)-\mathrm{C}(2)-\mathrm{C}(3)$ & $109.5(5)$
\end{tabular}


$\mathrm{C}(11)-\mathrm{C}(2)-\mathrm{S}(1)$

$C(3)-C(2)-S(1)$

$C(2)-C(3)-C(4)$

$C(2)-C(3)-S(2)$

$C(4)-C(3)-S(2)$

$\mathrm{N}(2)-\mathrm{C}(4)-\mathrm{C}(3)$

$\mathrm{N}(2)-\mathrm{C}(4)-\mathrm{C}(5)$

$C(3)-C(4)-C(5)$

$C(6)-C(5)-C(9)$

$C(6)-C(5)-C(4)$

$C(9)-C(5)-C(4)$

$\mathrm{C}(7)-\mathrm{C}(6)-\mathrm{C}(5)$

$\mathrm{C}(7)-\mathrm{C}(6)-\mathrm{H}(6)$

$\mathrm{C}(5)-\mathrm{C}(6)-\mathrm{H}(6)$

$\mathrm{N}(1)-\mathrm{C}(7)-\mathrm{C}(6)$

$\mathrm{N}(1)-\mathrm{C}(7)-\mathrm{H}(7)$

$\mathrm{C}(6)-\mathrm{C}(7)-\mathrm{H}(7)$

$\mathrm{N}(1)-\mathrm{C}(8)-\mathrm{C}(9)$

$\mathrm{N}(1)-\mathrm{C}(8)-\mathrm{H}(8)$

$\mathrm{C}(9)-\mathrm{C}(8)-\mathrm{H}(8)$

$C(8)-C(9)-C(5)$

$\mathrm{C}(8)-\mathrm{C}(9)-\mathrm{H}(9)$

$\mathrm{C}(5)-\mathrm{C}(9)-\mathrm{H}(9)$

$\mathrm{N}(1)-\mathrm{C}(10)-\mathrm{H}(10 \mathrm{~A})$

$\mathrm{N}(1)-\mathrm{C}(10)-\mathrm{H}(10 \mathrm{~B})$

$\mathrm{H}(10 \mathrm{~A})-\mathrm{C}(10)-\mathrm{H}(10 \mathrm{~B})$

$\mathrm{N}(1)-\mathrm{C}(10)-\mathrm{H}(10 \mathrm{C})$

$\mathrm{H}(10 \mathrm{~A})-\mathrm{C}(10)-\mathrm{H}(10 \mathrm{C})$

$\mathrm{H}(10 \mathrm{~B})-\mathrm{C}(10)-\mathrm{H}(10 \mathrm{C})$

$\mathrm{N}(2)-\mathrm{C}(11)-\mathrm{C}(2)$

$\mathrm{N}(2)-\mathrm{C}(11)-\mathrm{C}(12)$

$C(2)-C(11)-C(12)$

$C(16)-C(12)-C(13)$

$C(16)-C(12)-C(11)$

C (13) $-\mathrm{C}(12)-\mathrm{C}(11)$

$C(14)-C(13)-C(12)$

$\mathrm{C}(14)-\mathrm{C}(13)-\mathrm{H}(13)$

$\mathrm{C}(12)-\mathrm{C}(13)-\mathrm{H}(13)$

$\mathrm{N}(3)-\mathrm{C}(14)-\mathrm{C}(13)$

$\mathrm{N}(3)-\mathrm{C}(14)-\mathrm{H}(14)$

$\mathrm{C}(13)-\mathrm{C}(14)-\mathrm{H}(14)$

$\mathrm{N}(3)-\mathrm{C}(15)-\mathrm{C}(16)$

$\mathrm{N}(3)-\mathrm{C}(15)-\mathrm{H}(15)$

$\mathrm{C}(16)-\mathrm{C}(15)-\mathrm{H}(15)$

$C(15)-C(16)-C(12)$

$\mathrm{C}(15)-\mathrm{C}(16)-\mathrm{H}(16)$

$\mathrm{C}(12)-\mathrm{C}(16)-\mathrm{H}(16)$

$\mathrm{N}(3)-\mathrm{C}(17)-\mathrm{H}(17 \mathrm{~A})$

$\mathrm{N}(3)-\mathrm{C}(17)-\mathrm{H}(17 \mathrm{~B})$

$\mathrm{H}(17 \mathrm{~A})-\mathrm{C}(17)-\mathrm{H}(17 \mathrm{~B})$

$\mathrm{N}(3)-\mathrm{C}(17)-\mathrm{H}(17 \mathrm{C})$

$\mathrm{H}(17 \mathrm{~A})-\mathrm{C}(17)-\mathrm{H}(17 \mathrm{C})$

$\mathrm{H}(17 \mathrm{~B})-\mathrm{C}(17)-\mathrm{H}(17 \mathrm{C})$

$\mathrm{O}(1)-\mathrm{C}(18)-\mathrm{N}(4)$

$\mathrm{O}(1)-\mathrm{C}(18)-\mathrm{H}(18)$

$\mathrm{N}(4)-\mathrm{C}(18)-\mathrm{H}(18)$

$\mathrm{N}(4)-\mathrm{C}(19)-\mathrm{H}(19 \mathrm{~A})$

$\mathrm{N}(4)-\mathrm{C}(19)-\mathrm{H}(19 \mathrm{~B})$

$\mathrm{H}(19 \mathrm{~A})-\mathrm{C}(19)-\mathrm{H}(19 \mathrm{~B})$

$\mathrm{N}(4)-\mathrm{C}(19)-\mathrm{H}(19 \mathrm{C})$

$\mathrm{H}(19 \mathrm{~A})-\mathrm{C}(19)-\mathrm{H}(19 \mathrm{C})$

$\mathrm{H}(19 \mathrm{~B})-\mathrm{C}(19)-\mathrm{H}(19 \mathrm{C})$
$133.7(5)$

$116.8(4)$

$107.5(5)$

$118.8(5)$

$133.6(5)$

$105.9(5)$

$125.9(5)$

$127.9(6)$

$116.3(6)$

$121.4(6)$

$122.3(6)$

$121.7(6)$

119.2

119.2

$119.9(7)$

120.1

120.1

$121.6(6)$

119.2

119.2

$120.3(7)$

119.8

119.8

109.5

109.5

109.5

109.5

109.5

109.5

$105.8(5)$

$124.0(6)$

$130.2(6)$

$116.7(6)$

$121.2(6)$

$122.1(6)$

$119.0(7)$

120.5

120.5

$122.6(6)$

118.7

118.7

$120.1(7)$

120.0

120.0

$121.3(6)$

119.3

119.3

109.5

109.5

109.5

109.5

109.5

109.5

127.7 (11)

116.2

116.2

109.5

109.5

109.5

109.5

109.5

109.5 


\begin{tabular}{|c|c|}
\hline$N(4)-C(20)-H(20 A)$ & 109.5 \\
\hline $\mathrm{N}(4)-\mathrm{C}(20)-\mathrm{H}(20 \mathrm{~B})$ & 109.5 \\
\hline $\mathrm{H}(20 \mathrm{~A})-\mathrm{C}(20)-\mathrm{H}(20 \mathrm{~B})$ & 109.5 \\
\hline $\mathrm{N}(4)-\mathrm{C}(20)-\mathrm{H}(20 \mathrm{C})$ & 109.5 \\
\hline $\mathrm{H}(20 \mathrm{~A})-\mathrm{C}(20)-\mathrm{H}(20 \mathrm{C})$ & 109.5 \\
\hline $\mathrm{H}(20 \mathrm{~B})-\mathrm{C}(20)-\mathrm{H}(20 \mathrm{C})$ & 109.5 \\
\hline $\mathrm{O}(2)-\mathrm{C}(21)-\mathrm{N}(5)$ & $125.0(8)$ \\
\hline $\mathrm{O}(2)-\mathrm{C}(21)-\mathrm{H}(21)$ & 117.5 \\
\hline $\mathrm{N}(5)-\mathrm{C}(21)-\mathrm{H}(21)$ & 117.5 \\
\hline $\mathrm{N}(5)-\mathrm{C}(22)-\mathrm{H}(22 \mathrm{~A})$ & 109.5 \\
\hline$N(5)-C(22)-H(22 B)$ & 109.5 \\
\hline $\mathrm{H}(22 \mathrm{~A})-\mathrm{C}(22)-\mathrm{H}(22 \mathrm{~B})$ & 109.5 \\
\hline$N(5)-C(22)-H(22 C)$ & 109.5 \\
\hline $\mathrm{H}(22 \mathrm{~A})-\mathrm{C}(22)-\mathrm{H}(22 \mathrm{C})$ & 109.5 \\
\hline $\mathrm{H}(22 \mathrm{~B})-\mathrm{C}(22)-\mathrm{H}(22 \mathrm{C})$ & 109.5 \\
\hline$N(5)-C(23)-H(23 A)$ & 109.5 \\
\hline$N(5)-C(23)-H(23 B)$ & 109.5 \\
\hline $\mathrm{H}(23 \mathrm{~A})-\mathrm{C}(23)-\mathrm{H}(23 \mathrm{~B})$ & 109.5 \\
\hline$N(5)-C(23)-H(23 C)$ & 109.5 \\
\hline $\mathrm{H}(23 \mathrm{~A})-\mathrm{C}(23)-\mathrm{H}(23 \mathrm{C})$ & 109.5 \\
\hline $\mathrm{H}(23 \mathrm{~B})-\mathrm{C}(23)-\mathrm{H}(23 \mathrm{C})$ & 109.5 \\
\hline $\mathrm{C}(8)-\mathrm{N}(1)-\mathrm{C}(7)$ & $120.2(6)$ \\
\hline$C(8)-N(1)-C(10)$ & $120.8(6)$ \\
\hline$C(7)-N(1)-C(10)$ & $119.0(6)$ \\
\hline $\mathrm{C}(11)-\mathrm{N}(2)-\mathrm{C}(4)$ & $111.3(5)$ \\
\hline $\mathrm{C}(11)-\mathrm{N}(2)-\mathrm{H}(2)$ & 124.3 \\
\hline $\mathrm{C}(4)-\mathrm{N}(2)-\mathrm{H}(2)$ & 124.3 \\
\hline$C(14)-N(3)-C(15)$ & $120.2(6)$ \\
\hline $\mathrm{C}(14)-\mathrm{N}(3)-\mathrm{C}(17)$ & $119.6(6)$ \\
\hline$C(15)-N(3)-C(17)$ & $120.2(7)$ \\
\hline $\mathrm{C}(18)-\mathrm{N}(4)-\mathrm{C}(19)$ & $121.6(9)$ \\
\hline$C(18)-N(4)-C(20)$ & $117.8(9)$ \\
\hline$C(19)-N(4)-C(20)$ & $120.4(10)$ \\
\hline $\mathrm{C}(21)-\mathrm{N}(5)-\mathrm{C}(23)$ & $120.0(7)$ \\
\hline$C(21)-N(5)-C(22)$ & $123.0(8)$ \\
\hline$C(23)-N(5)-C(22)$ & $117.0(8)$ \\
\hline$F(4)-P(1)-F(1)$ & $92.5(4)$ \\
\hline$F(4)-P(1)-F(6)$ & $89.5(4)$ \\
\hline$F(1)-P(1)-F(6)$ & $177.5(4)$ \\
\hline$F(4)-P(1)-F(5)$ & $92.4(4)$ \\
\hline$F(1)-P(1)-F(5)$ & $90.1(3)$ \\
\hline$F(6)-P(1)-F(5)$ & $91.3(3)$ \\
\hline$F(4)-P(1)-F(2)$ & $90.8(4)$ \\
\hline$F(1)-P(1)-F(2)$ & $89.6(3)$ \\
\hline$F(6)-P(1)-F(2)$ & $88.9(3)$ \\
\hline$F(5)-P(1)-F(2)$ & $176.8(4)$ \\
\hline$F(4)-P(1)-F(3)$ & $179.2(4)$ \\
\hline$F(1)-P(1)-F(3)$ & $88.2(4)$ \\
\hline$F(6)-P(1)-F(3)$ & $89.8(4)$ \\
\hline$F(5)-P(1)-F(3)$ & $88.0(4)$ \\
\hline$F(2)-P(1)-F(3)$ & $88.8(4)$ \\
\hline$F(10)-P(2)-F(7)$ & $90.6(5)$ \\
\hline$F(10)-P(2)-F(8)$ & $177.7(7)$ \\
\hline$F(7)-P(2)-F(8)$ & $89.2(5)$ \\
\hline$F(10)-P(2)-F(9)$ & $95.5(6)$ \\
\hline$F(7)-P(2)-F(9)$ & $90.4(4)$ \\
\hline$F(8)-P(2)-F(9)$ & $86.8(6)$ \\
\hline$F(10)-P(2)-F(12)$ & $92.6(5)$ \\
\hline$F(7)-P(2)-F(12)$ & $176.6(5)$ \\
\hline$F(8)-P(2)-F(12)$ & $87.6(5)$ \\
\hline$F(9)-P(2)-F(12)$ & $90.5(4)$ \\
\hline$F(10)-P(2)-F(11)$ & $86.5(6)$ \\
\hline
\end{tabular}




$$
\begin{array}{lr}
F(7)-P(2)-F(11) & 90.4(3) \\
F(8)-P(2)-F(11) & 91.2(6) \\
F(9)-P(2)-F(11) & 177.8(5) \\
F(12)-P(2)-F(11) & 88.5(3) \\
C(2)-S(1)-C(1) & 93.9(3) \\
C(3)-S(2)-C(1) & 93.7(3)
\end{array}
$$

\begin{tabular}{|c|c|c|c|c|c|c|}
\hline & $\mathrm{U11}$ & $\mathrm{U} 22$ & U33 & $\mathrm{U} 23$ & $\mathrm{U} 13$ & \\
\hline$C(1)$ & $0.029(4)$ & $0.029(4)$ & $0.038(4)$ & $0.000(3)$ & $0.008(3)$ & \\
\hline $0.000(3)$ & & & & & & \\
\hline $\begin{array}{c}C(2) \\
0.007(3)\end{array}$ & $0.028(4)$ & $0.026(4)$ & $0.032(4)$ & $0.006(3)$ & $0.008(3)$ & - \\
\hline $\begin{array}{c}C(3) \\
0.004(3)\end{array}$ & $0.028(4)$ & $0.028(4)$ & $0.030(4)$ & $0.004(3)$ & $0.010(3)$ & - \\
\hline $\begin{array}{c}C(4) \\
0.001(3)\end{array}$ & $0.031(4)$ & $0.026(4)$ & $0.032(4)$ & $0.004(3)$ & $0.009(3)$ & - \\
\hline $\begin{array}{c}C(5) \\
0.001(3)\end{array}$ & $0.025(3)$ & $0.033(4)$ & $0.034(4)$ & $0.007(3)$ & $0.006(3)$ & \\
\hline $\begin{array}{c}C(6) \\
0.004(3)\end{array}$ & $0.041(4)$ & $0.032(4)$ & $0.041(4)$ & $-0.001(3)$ & $0.022(3)$ & \\
\hline $\begin{array}{c}C(7) \\
0.002(3)\end{array}$ & $0.037(4)$ & $0.038(4)$ & $0.049(5)$ & $0.000(4)$ & $0.009(4)$ & \\
\hline $\begin{array}{c}C(8) \\
0.000(4)\end{array}$ & $0.044(5)$ & $0.051(5)$ & $0.051(5)$ & $-0.002(4)$ & $0.027(4)$ & \\
\hline $\begin{array}{c}C(9) \\
0.000(4)\end{array}$ & $0.044(4)$ & $0.034(4)$ & $0.058(5)$ & $-0.003(4)$ & $0.027(4)$ & \\
\hline $\begin{array}{c}C(10) \\
0.015(4)\end{array}$ & $0.065(5)$ & $0.050(5)$ & $0.046(5)$ & $-0.012(4)$ & $0.020(4)$ & \\
\hline $\begin{array}{c}C(11) \\
0.001(3)\end{array}$ & $0.028(4)$ & $0.027(4)$ & $0.031(4)$ & $0.006(3)$ & $0.006(3)$ & - \\
\hline $\begin{array}{c}C(12) \\
0.006(3)\end{array}$ & $0.036(4)$ & $0.028(4)$ & $0.035(4)$ & $0.005(3)$ & $0.005(3)$ & - \\
\hline $\begin{array}{c}C(13) \\
0.012(3)\end{array}$ & $0.045(4)$ & $0.034(4)$ & $0.045(4)$ & $0.001(3)$ & $0.014(4)$ & - \\
\hline $\begin{array}{c}C(14) \\
0.017(4)\end{array}$ & $0.045(5)$ & $0.039(5)$ & $0.052(5)$ & $0.004(4)$ & $0.013(4)$ & - \\
\hline $\begin{array}{c}C(15) \\
0.008(4)\end{array}$ & $0.044(4)$ & $0.035(4)$ & $0.044(4)$ & $-0.004(4)$ & $0.007(4)$ & - \\
\hline $\begin{array}{c}C(16) \\
0.010(3)\end{array}$ & $0.040(4)$ & $0.035(4)$ & $0.039(4)$ & $-0.003(3)$ & $0.007(3)$ & - \\
\hline $\begin{array}{c}C(17) \\
0.022(4)\end{array}$ & $0.074(6)$ & $0.030(4)$ & $0.067(6)$ & $-0.005(4)$ & $0.004(5)$ & - \\
\hline $\begin{array}{c}C(18) \\
0.015(5)\end{array}$ & $0.081(7)$ & $0.042(6)$ & $0.098(9)$ & $-0.001(6)$ & $0.015(7)$ & - \\
\hline $\begin{array}{c}C(19) \\
0.004(7)\end{array}$ & $0.127(10)$ & $0.088(8)$ & $0.096(9)$ & $-0.016(7)$ & $0.053(8)$ & - \\
\hline $\begin{array}{c}C(20) \\
0.043(8)\end{array}$ & $0.082(9)$ & $0.122(11)$ & $0.142(12)$ & $-0.024(9)$ & $-0.011(8)$ & \\
\hline $\begin{array}{c}C(21) \\
0.002(5)\end{array}$ & $0.068(6)$ & $0.053(5)$ & $0.054(5)$ & $-0.002(4)$ & $0.011(5)$ & \\
\hline $\begin{array}{c}C(22) \\
0.034(6)\end{array}$ & $0.065(6)$ & $0.109(9)$ & $0.086(7)$ & $0.017(7)$ & $0.025(6)$ & - \\
\hline C (23) & $0.085(8)$ & $0.095(9)$ & $0.114(10)$ & $-0.034(8)$ & $0.030(7)$ & - \\
\hline
\end{tabular}

Symmetry transformations used to generate equivalent atoms:

$$
\# 1-x+1,-y+2,-z
$$

Table S14. Anisotropic displacement parameters for $\mathbf{5}$.

The anisotropic displacement factor exponent takes the form:

$-2 \mathrm{pi}^{\wedge} 2\left[\mathrm{~h}^{\wedge} 2 \mathrm{a}^{* \wedge} 2 \mathrm{U} 11+\ldots+2 \mathrm{~h} \mathrm{k} \mathrm{a}^{*} \mathrm{~b}^{*} \mathrm{U} 12\right]$ 
$0.014(7)$ F (1)

0.020 (3) $\mathrm{F}(2)$

$0.034(4)$ F (3)

0.033 (5) $\mathrm{F}(4)$

0.060 (5) $\mathrm{F}(5)$

0.018 (3) $\mathrm{F}(6)$

$0.006(3)$ $\mathrm{F}(7)$

0.045 (5) F (8)

0.039 (6) F (9)

$0.074(5)$ F (10)

0.077 (7) F (11)

0.074 (5) F (12)

0.024 (4) $\mathrm{N}(1)$

0.012 (3) $\mathrm{N}(2)$

0.005 (2) $\mathrm{N}(3)$

0.011 (3) $\mathrm{N}(4)$

0.000 (4) $\mathrm{N}(5)$

0.011 (3) O (1)

0.001 (4) $\mathrm{O}(2)$

0.027 (3) $\mathrm{P}(1)$

0.0041 (10) $\mathrm{P}(2)$

$0.0046(10)$ $\mathrm{S}(1)$

0.0065 (8)

$\mathrm{S}(2)$

$0.0078(8)$

\begin{tabular}{|c|c|c|c|c|c|}
\hline $0.106(4)$ & $0.049(3)$ & $0.109(5)$ & $0.027(3)$ & $-0.043(4)$ & - \\
\hline $0.075(4)$ & $0.112(5)$ & $0.109(5)$ & $0.050(4)$ & $-0.040(3)$ & - \\
\hline $0.130(6)$ & $0.137(6)$ & $0.063(4)$ & $-0.007(4)$ & $0.016(4)$ & \\
\hline $0.165(7)$ & $0.125(6)$ & $0.102(5)$ & $-0.002(4)$ & $0.046(5)$ & \\
\hline $0.056(3)$ & $0.084(4)$ & $0.147(6)$ & $0.042(4)$ & $-0.020(3)$ & - \\
\hline $0.081(4)$ & $0.064(4)$ & $0.105(4)$ & $0.033(3)$ & $-0.010(3)$ & - \\
\hline $0.084(4)$ & $0.218(8)$ & $0.069(4)$ & $-0.038(5)$ & $0.005(3)$ & \\
\hline $0.242(11)$ & $0.099(6)$ & $0.226(10)$ & $0.003(6)$ & $0.128(9)$ & - \\
\hline $0.091(5)$ & $0.176(7)$ & $0.146(6)$ & $-0.086(6)$ & $-0.050(4)$ & \\
\hline $0.214(10)$ & $0.130(7)$ & $0.204(10)$ & $0.028(7)$ & $0.030(8)$ & - \\
\hline $0.085(4)$ & $0.250(10)$ & $0.074(4)$ & $-0.042(5)$ & $-0.012(3)$ & \\
\hline $0.065(4)$ & $0.219(8)$ & $0.068(4)$ & $-0.043(5)$ & $-0.008(3)$ & \\
\hline $0.033(3)$ & $0.042(4)$ & $0.036(3)$ & $-0.003(3)$ & $0.004(3)$ & \\
\hline $0.030(3)$ & $0.029(3)$ & $0.039(3)$ & $0.000(3)$ & $0.014(3)$ & - \\
\hline $0.050(4)$ & $0.028(3)$ & $0.039(3)$ & $0.001(3)$ & $0.001(3)$ & - \\
\hline $0.054(5)$ & $0.052(5)$ & $0.078(6)$ & $-0.012(4)$ & $-0.002(4)$ & \\
\hline $0.050(4)$ & $0.051(4)$ & $0.048(4)$ & $-0.003(3)$ & $0.023(3)$ & - \\
\hline $0.089(5)$ & $0.083(5)$ & $0.081(5)$ & $-0.008(4)$ & $0.028(4)$ & - \\
\hline $0.045(3)$ & $0.065(4)$ & $0.073(4)$ & $-0.003(3)$ & $0.031(3)$ & - \\
\hline $0.0526(13)$ & $0.0354(12)$ & $0.0508(13)$ & $0.0071(10)$ & $-0.0060(10)$ & - \\
\hline $0.0372(11)$ & $0.0609(14)$ & $0.0356(11)$ & $0.0026(10)$ & $0.0074(9)$ & - \\
\hline $0.0432(11)$ & $0.0255(9)$ & $0.0466(11)$ & $-0.0028(9)$ & $0.0243(9)$ & - \\
\hline $0.0381(10)$ & $0.0280(9)$ & $0.0464(11)$ & $-0.0032(9)$ & $0.0213(9)$ & - \\
\hline
\end{tabular}




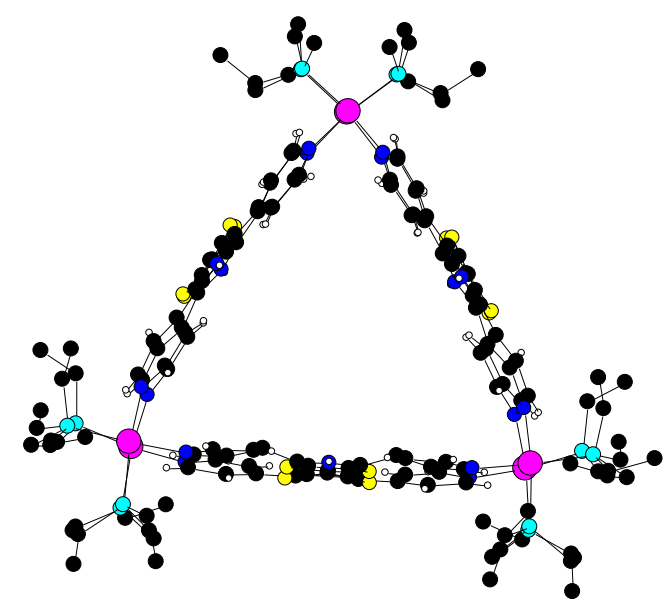

Figure S33. X-Ray structure of prisme $\mathbf{6 a}$

Table S15. Crystal data and structure refinement for $\mathbf{6 a}$

Empirical formula

Formula weight

Temperature

Wavelength

Crystal system, space group

Unit cell dimensions

\section{Volume}

Z, Calculated density

Absorption coefficient

$\mathrm{F}(000)$

Crystal size

Theta range for data collection

Limiting indices

Reflections collected / unique

Completeness to theta $=25.00$

Absorption correction

Max. and min. transmission

Refinement method

Data / restraints / parameters

Goodness-of-fit on $\mathrm{F}^{\wedge} 2$

Final R indices [I>2sigma(I)]

$\mathrm{R}$ indices (all data)

Largest diff. peak and hole
C162 H234 N18 075 P12 Pt6 S12

5560.57

200(2) K

$0.71073 \mathrm{~A}$

Orthorhombic, P n m a

$\mathrm{a}=41.62(1) \mathrm{A}$ alpha $=90 \mathrm{deg}$.

$\mathrm{b}=34.203(4) \mathrm{A} \quad$ beta $=90 \mathrm{deg}$.

$\mathrm{c}=19.61(1) \mathrm{A}$ gamma $=90 \mathrm{deg}$.

27915(16) $A^{\wedge} 3$

4, $1.323 \mathrm{Mg} / \mathrm{m}^{\wedge} 3$

$3.223 \mathrm{~mm}^{\wedge}-1$

11088

$0.3 \times 0.18 \times 0.1 \mathrm{~mm}$

2.04 to $25.21 \mathrm{deg}$.

$-49<=\mathrm{h}<=49,-27<=\mathrm{k}<=40,-21<=\mathrm{l}<=2$

$99343 / 22102$ [R(int) $=0.2007]$

$87.2 \%$

Semi-empirical from equivalents

0.724 and 0.281

Full-matrix least-squares on $\mathrm{F}^{\wedge} 2$

22102 / 8 / 680

1.927

$\mathrm{R} 1=0.1862, \mathrm{wR} 2=0.4577 \quad[6742 \mathrm{Fo}]$

$\mathrm{R} 1=0.3974, \mathrm{wR} 2=0.5863$

4.356 and -3.022 e. $A^{\wedge}-3$

Table S16. Atomic coordinates and equivalent isotropic displacement parameters for $\mathbf{6 a}$.

$\mathrm{U}(\mathrm{eq})$ is defined as one third of the trace of the orthogonalized Uij tensor.

\begin{tabular}{ccccc}
\hline & $x$ & $y$ & $z$ & U(eq) \\
\hline C (1) & $0.2690(10)$ & $0.5538(12)$ & $0.267(2)$ & $0.078(12)$ \\
$C(2)$ & $0.2740(9)$ & $0.5677(10)$ & $0.2060(18)$ & $0.067(10)$ \\
$C(3)$ & $0.2538(9)$ & $0.5918(11)$ & $0.1734(19)$ & $0.069(10)$ \\
$C(4)$ & $0.2252(10)$ & $0.5982(11)$ & $0.2016(19)$ & $0.077(11)$
\end{tabular}


C (5)

C (6)

C (7)

C (8)

$\mathrm{C}(9)$

C (10)

C (11)

C (12)

C (13)

C (14)

$\mathrm{C}(15)$

C (16)

$\mathrm{C}(17)$

C (18)

C (19)

C (20)

$\mathrm{C}(21)$

C (22)

C (23)

C (24)

C (25)

C (26)

C (27)

$\mathrm{C}(28)$

C (29)

C (30)

C (31)

C (32)

C (33)

C (34)

C (35)

C (36)

C (37)

C (38)

C (39)

C (40)

C (41)

C (42)

C (43)

C (44)

C (45)

C ( 46$)$

C (47)

C (48)

C (49)

C (50)

C (51)

C (52)

C (53)

C (54)

C (55)

C (56)

C (57)

C (58)

C (59)

C (60)

C (61)

C (62)

C (63)

C (64)

C (65)

C (66)
0.2193 (11)

0.2591 (8)

$0.2869(9)$

$0.2812(9)$

$0.2482(8)$

$0.2336(8)$

$0.2016(10)$

0.1906 (11)

$0.2363(12)$

0.2477 (10)

0.3415 (10)

$0.3720(11)$

$0.4258(10)$

0.4315 (9)

0.4659 (11)

0.4831 (10)

0.5162 (11)

0.5338 (10)

0.4812 (13)

0.4668 (10)

0.4561 (10)

0.4637 (9)

$0.4940(12)$

0.4935 (15)

0.4428 (12)

$0.4397(9)$

$0.2832(10)$

$0.2989(10)$

0.2786 (9)

0.2451 (10)

$0.2340(9)$

$0.2896(12)$

0.3250 (11)

$0.3808(16)$

0.4115 (12)

0.4660 (9)

0.4957 (10)

0.5052

0.5413 (10)

0.5462 (11)

$0.4998(10)$

0.4859 (10)

$0.244(2)$

$0.2686(9)$

0.1950 (17)

0.182 (2)

0.1762 (16)

0.1471 (18)

0.2458 (15)

$0.2809(18)$

$0.1894(12)$

$0.1773(16)$

0.2530 (13)

0.5391 (13)

$0.519(4)$

0.5996 (14)

0.6297 (9)

0.571 (3)

0.591 (3)

$0.5216(12)$

$0.4888(12)$

0.5866 (13)
$0.5794(12)$

0.265 (2)

0.1057 (17)

0.0725 (17)

$0.0191(17)$

$0.0142(16)$

$0.6331(10)$

0.6431 (9)

$0.6636(9)$

0.6772 (11)

0.7001 (12)

$0.6952(13)$

$0.6746(11)$

$0.6186(11)$

0.6167 (12)

0.6276 (11)

$0.6087(11)$

$0.6028(13)$

0.5849 (11)

$0.5943(12)$

0.5752 (11)

0.5484 (14)

$0.5626(11)$

0.6401 (12)

$0.6633(10)$

0.6771 (12)

$0.7029(16)$

$0.6962(13)$

$0.6747(10)$

$0.6061(10)$

$0.6455(11)$

$0.6767(10)$

0.6693 (12)

$0.6343(10)$

$0.7175(14)$

0.7288 (11)

0.7500

0.7500

$0.7291(10)$

$0.7174(10)$

0.6808

$0.6714(12)$

$0.6294(13)$

$0.6103(11)$

$0.6442(12)$

0.448 (2)

$0.4510(10)$

$0.4853(18)$

0.4477 (19)

0.4701 (18)

$0.499(2)$

$0.4936(17)$

$0.4906(19)$

0.5446 (13)

0.5858 (19)

$0.5749(15)$

$0.5682(15)$

$0.577(5)$

0.5647 (17)

$0.5424(10)$

$0.495(3)$

0.492 (3)

$0.4419(17)$

$0.4618(14)$

0.4618 (14)
$-0.0389(17)$

$-0.034(2)$

$-0.083(2)$

$-0.150(2)$

$-0.098(2)$

$0.0100(19)$

$-0.001(2)$

$-0.055(2)$

0.0035 (18)

$0.016(2)$

$0.063(2)$

$0.076(2)$

0.1317 (19)

$0.172(3)$

$0.115(2)$

$-0.085(2)$

$-0.1396(18)$

$-0.154(2)$

$-0.215(3)$

$-0.238(2)$

$-0.1802(17)$

$0.4172(16)$

$0.4191(18)$

$0.4234(17)$

$0.4242(19)$

$0.4203(16)$

$0.419(2)$

$0.401(2)$

$0.372(3)$

0.357 (2)

0.3283 (19)

0.3097 (18)

0.2842

0.275 (2)

$0.3053(17)$

0.3098 (19)

$0.376(5)$

$0.3334(18)$

0.292 (3)

0.258 (4)

0.427 (3)

0.427 (3)

0.552 (3)

0.545 (3)

$0.554(2)$

$0.530(3)$

$0.563(3)$

$0.433(3)$

$0.497(6)$

$0.382(3)$

$0.3230(17)$

$0.434(6)$

$0.481(5)$

0.295 (3)

0.307 (3)

0.259 (3)
0.2742 (19)

$0.084(12)$

$0.058(9)$

$0.062(9)$

$0.062(9)$

$0.050(8)$

0.057 (9)

0.077 (11)

0.085 (12)

$0.093(13)$

$0.082(12)$

$0.078(11)$

$0.093(13)$

$0.079(11)$

$0.074(11)$

0.093 (13)

$0.081(12)$

$0.093(13)$

$0.079(11)$

$0.119(17)$

$0.081(12)$

$0.083(12)$

$0.069(10)$

$0.096(13)$

0.125 (18)

$0.100(14)$

$0.064(10)$

$0.064(10)$

$0.082(12)$

$0.066(10)$

0.081 (12)

$0.059(9)$

0.108 (15)

$0.094(13)$

0.091 (18)

0.060 (13)

$0.074(11)$

$0.067(10)$

$0.16(2)$

$0.084(12)$

0.092 (13) 


\begin{tabular}{|c|c|c|c|c|}
\hline C (67) & $0.5913(13)$ & $0.4146(15)$ & $0.229(3)$ & $0.120(17)$ \\
\hline$C(68)$ & $0.5339(18)$ & $0.470(2)$ & $0.166(4)$ & $0.17(2)$ \\
\hline C (69) & $0.5526(16)$ & $0.4772(18)$ & $0.102(3)$ & $0.15(2)$ \\
\hline$C(70)$ & $0.5205(16)$ & $0.711(3)$ & $-0.469(4)$ & $0.24(4)$ \\
\hline C (71) & $0.4867(17)$ & $0.6532(11)$ & $-0.363(3)$ & $0.17(3)$ \\
\hline C ( 72$)$ & $0.4489(16)$ & $0.691(4)$ & $-0.450(6)$ & $0.33(6)$ \\
\hline$C(73)$ & $0.1780(13)$ & $0.6541(15)$ & $-0.251(3)$ & $0.117(16)$ \\
\hline$C(74)$ & $0.1690(13)$ & $0.6167(15)$ & $-0.295(3)$ & $0.120(17)$ \\
\hline$C(75)$ & $0.1254(10)$ & $0.688(2)$ & $-0.248(4)$ & $0.20(3)$ \\
\hline$C(76)$ & $0.183(3)$ & $0.695(3)$ & $-0.365(2)$ & $0.28(5)$ \\
\hline $\mathrm{N}(1)$ & $0.2346(8)$ & $0.6291(9)$ & $0.0704(16)$ & $0.085(10)$ \\
\hline $\mathrm{N}(2)$ & $0.2381(8)$ & $0.5600(9)$ & $0.306(2)$ & $0.112(15)$ \\
\hline$N(3)$ & $0.2056(9)$ & $0.7114(11)$ & $-0.1390(19)$ & $0.108(13)$ \\
\hline$N(4)$ & $0.4777(7)$ & $0.6233(8)$ & $-0.0379(14)$ & $0.068(9)$ \\
\hline $\mathrm{N}(5)$ & $0.5178(9)$ & $0.5521(10)$ & $0.1793(17)$ & $0.091(10)$ \\
\hline$N(6)$ & $0.4748(9)$ & $0.7118(10)$ & $-0.2569(17)$ & $0.087(10)$ \\
\hline $\mathrm{N}(7)$ & $0.2552(7)$ & $0.6029(8)$ & $0.4185(17)$ & $0.079(10)$ \\
\hline $\mathrm{N}(8)$ & $0.5290(7)$ & $0.6021(6)$ & $0.2901(15)$ & $0.064(8)$ \\
\hline$N(9)$ & $0.5131(11)$ & 0.7500 & $0.301(3)$ & $0.14(3)$ \\
\hline$N(10)$ & $0.2728(9)$ & 0.7500 & $0.418(2)$ & $0.072(13)$ \\
\hline$P(1)$ & $0.2127(4)$ & $0.4880(3)$ & $0.3764(7)$ & $0.113(5)$ \\
\hline$P(2)$ & $0.2296(4)$ & $0.5390(4)$ & $0.5196(7)$ & $0.111(5)$ \\
\hline$P(3)$ & $0.5614(4)$ & $0.5398(3)$ & $0.3787(8)$ & $0.121(5)$ \\
\hline P ( 4) & $0.5455(3)$ & $0.4780(3)$ & $0.2468(9)$ & $0.118(5)$ \\
\hline$P(5)$ & $0.4880(5)$ & 0.7001 (4) & $-0.4077(8)$ & $0.128(5)$ \\
\hline$P(6)$ & $0.1670(5)$ & 0.7005 (4) & $-0.2763(9)$ & $0.139(6)$ \\
\hline Pt (1) & $0.23402(4)$ & $0.54606(4)$ & $0.40453(9)$ & $0.0755(6)$ \\
\hline Pt (2) & $0.53970(4)$ & $0.54232(4)$ & $0.27134(10)$ & $0.0846(7)$ \\
\hline Pt (3) & $0.48132(7)$ & 0.7500 & $-0.33216(13)$ & $0.0921(9)$ \\
\hline Pt (4) & $0.18466(7)$ & 0.7500 & $-0.20994(12)$ & $0.0859(9)$ \\
\hline$O(1)$ & $0.5602(5)$ & $0.5949(6)$ & $0.8822(11)$ & $0.055(6)$ \\
\hline$O(2)$ & $0.6299(7)$ & $0.6067(8)$ & $0.1585(13)$ & $0.082(8)$ \\
\hline$O(3)$ & $0.5824(9)$ & $0.5531(11)$ & $0.9748(19)$ & $0.138(12)$ \\
\hline$O(4)$ & $0.4130(8)$ & $0.5710(10)$ & $0.3276(17)$ & $0.120(11)$ \\
\hline$O(5)$ & $0.1779(16)$ & 0.7500 & $0.461(3)$ & $0.146(19)$ \\
\hline$O(6)$ & $0.5314(11)$ & $0.5377(12)$ & $0.940(2)$ & $0.166(15)$ \\
\hline$O(7)$ & $0.5458(9)$ & $0.6202(10)$ & $0.9289(18)$ & $0.132(12)$ \\
\hline$O(8)$ & $0.486(2)$ & $0.686(2)$ & $0.126(4)$ & $0.33(4)$ \\
\hline$O(9)$ & $0.288(2)$ & $0.685(2)$ & $0.221(4)$ & $0.32(4)$ \\
\hline$O(10)$ & $0.5923(12)$ & $0.6074(13)$ & $0.862(2)$ & $0.175(16)$ \\
\hline$O(11)$ & $0.590(2)$ & 0.7500 & $0.438(5)$ & $0.27(4)$ \\
\hline$O(12)$ & $0.6062(11)$ & $0.6209(13)$ & $0.565(2)$ & $0.181(17)$ \\
\hline$O(13)$ & $0.3245(16)$ & $0.4986(17)$ & $0.205(3)$ & $0.22(2)$ \\
\hline$O(14)$ & $0.415(2)$ & 0.7500 & $0.948(4)$ & $0.25(4)$ \\
\hline$O(15)$ & $0.3152(7)$ & $0.6617(8)$ & $0.2901(14)$ & 0.101 (9) \\
\hline$O(16)$ & $0.5797(17)$ & 0.7500 & 0.255 (3) & $0.18(2)$ \\
\hline$O(17)$ & $0.566(3)$ & 0.7500 & $0.923(6)$ & $0.28(4)$ \\
\hline$O(18)$ & $0.3543(14)$ & $0.5846(15)$ & $0.589(3)$ & $0.22(2)$ \\
\hline O (19) & $0.560(2)$ & $0.537(2)$ & $0.856(4)$ & $0.29(3)$ \\
\hline$O(20)$ & $0.6584(12)$ & $0.5896(13)$ & $0.133(2)$ & $0.185(17)$ \\
\hline$O(21)$ & 0.1695 (14) & $0.6261(15)$ & $0.091(3)$ & $0.22(2)$ \\
\hline$O(22)$ & $0.2119(19)$ & 0.7500 & $0.469(3)$ & $0.19(2)$ \\
\hline$O(23)$ & $0.6480(12)$ & $0.5774(14)$ & $0.571(2)$ & $0.193(18)$ \\
\hline$O(24)$ & $0.142(3)$ & 0.7500 & $0.373(6)$ & $0.32(5)$ \\
\hline$O(25)$ & $0.3264(12)$ & $0.5266(13)$ & $0.402(2)$ & $0.184(17)$ \\
\hline$O(26)$ & $0.5428(14)$ & $0.5904(17)$ & $0.838(3)$ & $0.22(2)$ \\
\hline$O(27)$ & $0.380(3)$ & 0.7500 & $0.846(6)$ & $0.33(5)$ \\
\hline$O(28)$ & $0.558(2)$ & 0.7500 & $0.858(5)$ & $0.25(4)$ \\
\hline$O(29)$ & $0.183(5)$ & 0.7500 & $0.382(9)$ & $0.46(9)$ \\
\hline$O(30)$ & $0.1522(16)$ & 0.7500 & $0.501(3)$ & $0.17(2)$ \\
\hline$O(31)$ & $0.450(3)$ & 0.7500 & $0.969(5)$ & $0.30(5)$ \\
\hline$O(32)$ & $0.294(2)$ & 0.7500 & $0.003(4)$ & $0.25(4)$ \\
\hline
\end{tabular}




\begin{tabular}{|c|c|c|c|c|}
\hline$O(33)$ & $0.4241(17)$ & $0.5986(19)$ & $0.383(3)$ & $0.27(3)$ \\
\hline$O(34)$ & $0.3521(17)$ & $0.4884(19)$ & $0.231(3)$ & $0.24(3)$ \\
\hline$O(35)$ & $0.6159(16)$ & $0.6171(18)$ & $0.035(3)$ & $0.26(3)$ \\
\hline$O(36)$ & $0.4212(16)$ & $0.5449(15)$ & $0.246(3)$ & $0.23(2)$ \\
\hline$O(37)$ & $0.3650(15)$ & $0.5795(16)$ & $0.256(3)$ & $0.22(2)$ \\
\hline$O(38)$ & $0.3264(18)$ & $0.627(2)$ & $0.614(3)$ & $0.28(3)$ \\
\hline$O(39)$ & $0.307(3)$ & $0.657(3)$ & $0.713(5)$ & $0.42(5)$ \\
\hline$O(40)$ & $0.400(3)$ & $0.605(3)$ & $0.257(5)$ & $0.38(5)$ \\
\hline$O(41)$ & $0.451(2)$ & $0.680(2)$ & $0.148(4)$ & $0.31(4)$ \\
\hline$O(42)$ & $0.599(2)$ & $0.599(2)$ & $0.638(4)$ & $0.31(3)$ \\
\hline$O(43)$ & $0.580(2)$ & $0.521(3)$ & $0.931(5)$ & $0.34(4)$ \\
\hline$O(44)$ & $0.6147(12)$ & $0.5853(15)$ & $0.175(2)$ & $0.183(18)$ \\
\hline$S(1)$ & $0.3148(3)$ & $0.6403(3)$ & $-0.0415(6)$ & $0.088(3)$ \\
\hline$S(2)$ & $0.3256(3)$ & $0.5922(4)$ & $0.0870(6)$ & $0.098(4)$ \\
\hline$S(3)$ & $0.3878(3)$ & $0.6353(5)$ & $-0.0732(7)$ & $0.125(5)$ \\
\hline$S(4)$ & $0.4006(3)$ & $0.5905(4)$ & $0.0525(7)$ & $0.114(5)$ \\
\hline$S(5)$ & $0.3578(3)$ & $0.7063(3)$ & $0.3968(9)$ & $0.120(5)$ \\
\hline$S(6)$ & $0.4308(3)$ & $0.7074(3)$ & $0.3470(9)$ & $0.121(5)$ \\
\hline $\mathrm{H}(1)$ & 0.2852 & 0.5392 & 0.2876 & 0.094 \\
\hline $\mathrm{H}(1 \mathrm{~A})$ & 0.2151 & 0.6329 & 0.0836 & 0.102 \\
\hline $\mathrm{H}(2)$ & 0.2928 & 0.5604 & 0.1837 & 0.080 \\
\hline $\mathrm{H}(4)$ & 0.2098 & 0.6140 & 0.1808 & 0.092 \\
\hline $\mathrm{H}(4 \mathrm{~A})$ & 0.4981 & 0.6261 & -0.0432 & 0.082 \\
\hline $\mathrm{H}(5)$ & 0.1982 & 0.5814 & 0.2805 & 0.101 \\
\hline $\mathrm{H}(9)$ & 0.5332 & 0.7500 & 0.2912 & 0.164 \\
\hline $\mathrm{H}(10)$ & 0.2522 & 0.7500 & 0.4177 & 0.086 \\
\hline $\mathrm{H}(11)$ & 0.1887 & 0.6702 & 0.0031 & 0.092 \\
\hline $\mathrm{H}(12)$ & 0.1699 & 0.7096 & -0.0767 & 0.102 \\
\hline $\mathrm{H}(13)$ & 0.2476 & 0.6987 & -0.1906 & 0.112 \\
\hline $\mathrm{H}(14)$ & 0.2689 & 0.6664 & -0.1029 & 0.099 \\
\hline $\mathrm{H}(21)$ & 0.5265 & 0.6126 & 0.0486 & 0.111 \\
\hline $\mathrm{H}(22)$ & 0.5559 & 0.5786 & 0.1353 & 0.095 \\
\hline $\mathrm{H}(23)$ & 0.4690 & 0.5366 & 0.2063 & 0.142 \\
\hline $\mathrm{H}(24)$ & 0.4450 & 0.5575 & 0.1084 & 0.097 \\
\hline $\mathrm{H}(27)$ & 0.5123 & 0.6711 & -0.1289 & 0.115 \\
\hline $\mathrm{H}(28)$ & 0.5132 & 0.7153 & -0.2212 & 0.151 \\
\hline H (29) & 0.4250 & 0.7013 & -0.2649 & 0.121 \\
\hline $\mathrm{H}(30)$ & 0.4190 & 0.6671 & -0.1678 & 0.077 \\
\hline $\mathrm{H}(31)$ & 0.2960 & 0.5838 & 0.4149 & 0.076 \\
\hline $\mathrm{H}(32)$ & 0.3211 & 0.6485 & 0.4174 & 0.098 \\
\hline H (34) & 0.2308 & 0.6902 & 0.4276 & 0.097 \\
\hline $\mathrm{H}(35)$ & 0.2119 & 0.6300 & 0.4186 & 0.070 \\
\hline H ( 43$)$ & 0.5574 & 0.6900 & 0.2683 & 0.101 \\
\hline $\mathrm{H}(44)$ & 0.5668 & 0.6220 & 0.2618 & 0.111 \\
\hline $\mathrm{H}(45)$ & 0.4867 & 0.5889 & 0.3144 & 0.079 \\
\hline H ( 46$)$ & 0.4652 & 0.6467 & 0.3276 & 0.101 \\
\hline
\end{tabular}

Table S17. Bond lengths [A] for $\mathbf{6 a}$.

$\begin{array}{ll}\mathrm{C}(1)-\mathrm{C}(2) & 1.31(5) \\ \mathrm{C}(1)-\mathrm{N}(2) & 1.51(5) \\ \mathrm{C}(1)-\mathrm{H}(1) & 0.9300 \\ \mathrm{C}(2)-\mathrm{C}(3) & 1.34(5) \\ \mathrm{C}(2)-\mathrm{H}(2) & 0.9300 \\ \mathrm{C}(3)-\mathrm{C}(4) & 1.33(5) \\ \mathrm{C}(3)-\mathrm{C}(6) & 1.43(5) \\ \mathrm{C}(4)-\mathrm{C}(5) & 1.43(5) \\ \mathrm{C}(4)-\mathrm{H}(4) & 0.9300\end{array}$




\begin{tabular}{|c|c|}
\hline$C(5)-N(2)$ & $1.30(5)$ \\
\hline $\mathrm{C}(5)-\mathrm{H}(5)$ & 0.9300 \\
\hline$C(6)-C(7)$ & $1.33(4)$ \\
\hline $\mathrm{C}(6)-\mathrm{N}(1)$ & $1.47(4)$ \\
\hline$C(7)-C(8)$ & $1.33(4)$ \\
\hline$C(7)-S(2)$ & $1.74(4)$ \\
\hline$C(8)-C(9)$ & $1.42(4)$ \\
\hline$C(8)-S(1)$ & $1.85(4)$ \\
\hline $\mathrm{C}(9)-\mathrm{N}(1)$ & $1.33(4)$ \\
\hline$C(9)-C(10)$ & $1.40(4)$ \\
\hline$C(10)-C(14)$ & $1.35(5)$ \\
\hline$C(10)-C(11)$ & $1.41(5)$ \\
\hline$C(11)-C(12)$ & $1.32(5)$ \\
\hline $\mathrm{C}(11)-\mathrm{H}(11)$ & 0.9300 \\
\hline $\mathrm{C}(12)-\mathrm{N}(3)$ & $1.33(5)$ \\
\hline $\mathrm{C}(12)-\mathrm{H}(12)$ & 0.9300 \\
\hline$C(13)-C(14)$ & $1.33(5)$ \\
\hline$C(13)-N(3)$ & $1.41(5)$ \\
\hline $\mathrm{C}(13)-\mathrm{H}(13)$ & 0.9300 \\
\hline $\mathrm{C}(14)-\mathrm{H}(14)$ & 0.9300 \\
\hline$C(15)-C(16)$ & $1.29(5)$ \\
\hline$C(15)-S(1)$ & $1.67(4)$ \\
\hline$C(15)-S(2)$ & $1.88(4)$ \\
\hline$C(16)-S(3)$ & $1.69(4)$ \\
\hline$C(16)-S(4)$ & $1.82(5)$ \\
\hline$C(17)-C(18)$ & $1.34(5)$ \\
\hline$C(17)-C(25)$ & $1.45(5)$ \\
\hline$C(17)-S(3)$ & $1.64(4)$ \\
\hline$C(18)-C(19)$ & $1.47(5)$ \\
\hline$C(18)-S(4)$ & $1.72(4)$ \\
\hline$C(19)-C(20)$ & $1.32(5)$ \\
\hline $\mathrm{C}(19)-\mathrm{N}(4)$ & $1.36(5)$ \\
\hline$C(20)-C(24)$ & $1.43(5)$ \\
\hline$C(20)-C(21)$ & $1.43(5)$ \\
\hline$C(21)-C(22)$ & $1.47(5)$ \\
\hline $\mathrm{C}(21)-\mathrm{H}(21)$ & 0.9300 \\
\hline$C(22)-N(5)$ & $1.39(5)$ \\
\hline $\mathrm{C}(22)-\mathrm{H}(22)$ & 0.9300 \\
\hline$C(23)-C(24)$ & $1.37(6)$ \\
\hline$C(23)-N(5)$ & $1.53(6)$ \\
\hline $\mathrm{C}(23)-\mathrm{H}(23)$ & 0.9300 \\
\hline $\mathrm{C}(24)-\mathrm{H}(24)$ & 0.9300 \\
\hline$C(25)-C(26)$ & $1.37(5)$ \\
\hline$C(25)-N(4)$ & $1.41(5)$ \\
\hline$C(26)-C(30)$ & $1.34(5)$ \\
\hline$C(26)-C(27)$ & $1.38(5)$ \\
\hline$C(27)-C(28)$ & $1.48(6)$ \\
\hline $\mathrm{C}(27)-\mathrm{H}(27)$ & 0.9300 \\
\hline$C(28)-N(6)$ & $1.18(6)$ \\
\hline $\mathrm{C}(28)-\mathrm{H}(28)$ & 0.9300 \\
\hline$C(29)-C(30)$ & $1.35(5)$ \\
\hline$C(29)-N(6)$ & $1.48(5)$ \\
\hline $\mathrm{C}(29)-\mathrm{H}(29)$ & 0.9300 \\
\hline $\mathrm{C}(30)-\mathrm{H}(30)$ & 0.9300 \\
\hline $\mathrm{C}(31)-\mathrm{N}(7)$ & $1.17(4)$ \\
\hline$C(31)-C(32)$ & $1.50(5)$ \\
\hline $\mathrm{C}(31)-\mathrm{H}(31)$ & 0.9300 \\
\hline$C(32)-C(33)$ & $1.36(5)$ \\
\hline $\mathrm{C}(32)-\mathrm{H}(32)$ & 0.9300 \\
\hline$C(33)-C(34)$ & $1.42(5)$ \\
\hline$C(33)-C(36)$ & $1.47(5)$ \\
\hline$C(34)-C(35)$ & $1.29(5)$ \\
\hline
\end{tabular}


$\mathrm{C}(34)-\mathrm{H}(34)$

0.9300

$\mathrm{C}(35)-\mathrm{N}(7)$

1.39(4)

C (35) $-\mathrm{H}(35)$

0.9300

$\mathrm{C}(36)-\mathrm{N}(10)$

$1.31(5)$

C (36) $-\mathrm{C}(37)$

$1.56(6)$

C (37) - C (37) \# 1

$1.45(8)$

$C(37)-S(5)$

$1.57(4)$

C (38) -C (39)

$1.31(7)$

C (38) $-S(5)$

$1.84(4)$

C (38) $-S(5)$ \# 1

$1.84(4)$

$C(39)-S(6)$

$1.67(3)$

C (39) $-S(6)$ \# 1

$1.67(3)$

C (40)-C (41)

$1.35(5)$

$C(40)-C(40) \# 1$

$1.43(7)$

$\mathrm{C}(40)-\mathrm{S}(6)$

$1.68(4)$

$\mathrm{C}(41)-\mathrm{N}(9)$

$1.34(4)$

C (41) $-\mathrm{C}(42)$

$1.41(4)$

$C(42)-C(43)$

1.55 (4)

C ( 42) $-\mathrm{C}(46)$

$1.57(4)$

C (43) $-\mathrm{C}(44)$

1. $45(5)$

$\mathrm{C}(43)-\mathrm{H}(43)$

0.9300

$\mathrm{C}(44)-\mathrm{N}(8)$

$1.22(5)$

$\mathrm{C}(44)-\mathrm{H}(44)$

0.9300

$\mathrm{C}(45)-\mathrm{N}(8)$

$1.28(4)$

$C(45)-C(46)$

$1.30(5)$

C (45) $-\mathrm{H}(45)$

0.9300

$\mathrm{C}(46)-\mathrm{H}(46)$

0.9300

C (47) $-\mathrm{C}(48)$

1. 31 (9)

$C(47)-P(1)$

$1.91(9)$

$C(49)-C(50)$

$1.54(2)$

$C(49)-P(1)$

$1.82(7)$

$C(51)-C(52)$

$1.56(8)$

$C(51)-P(1)$

$1.92(6)$

C (53) $-\mathrm{C}(54)$

$1.47(8)$

$C(53)-P(2)$

$1.81(6)$

$C(55)-C(56)$

$1.57(7)$

$C(55)-P(2)$

$1.81(5)$

$C(57)-P(2)$

$1.78(5)$

$C(58)-C(59)$

$1.54(2)$

$C(58)-P(3)$

$1.71(6)$

$C(60)-P(3)$

$1.80(6)$

$C(60)-C(61)$

$1.87(7)$

$C(62)-C(63)$

$1.25(13)$

$C(62)-P(3)$

1.92 (12)

$C(64)-C(65)$

$1.54(2)$

$C(64)-P(4)$

$1.84(6)$

$C(66)-C(67)$

$1.72(6)$

$\mathrm{C}(66)-\mathrm{P}(4)$

$1.82(6)$

$C(68)-C(69)$

$1.49(8)$

$C(68)-P(4)$

$1.68(7)$

$C(70)-P(5)$

$1.85(2)$

$C(71)-P(5)$

1.833 (19)

$\mathrm{C}(72)-\mathrm{P}(5)$

$1.85(2)$

$C(73)-C(74)$

$1.58(6)$

$\mathrm{C}(73)-\mathrm{P}(6)$

$1.72(5)$

$\mathrm{C}(75)-\mathrm{P}(6)$

$1.87(2)$

$\mathrm{C}(76)-\mathrm{P}(6)$

$1.86(2)$

$\mathrm{N}(1)-\mathrm{H}(1 \mathrm{~A})$

0.8600

$\mathrm{N}(2)-\mathrm{Pt}(1)$

$2.00(5)$

$\mathrm{N}(3)-\mathrm{Pt}(4)$

$2.11(3)$

0.8600

$\mathrm{N}(4)-\mathrm{H}(4 \mathrm{~A})$

$2.05(3)$ 


\begin{tabular}{|c|c|}
\hline$N(6)-P t(3)$ & $1.99(3)$ \\
\hline$N(7)-P t(1)$ & $2.15(3)$ \\
\hline$N(8)-P t(2)$ & $2.12(2)$ \\
\hline$N(9)-C(41) \# 1$ & $1.34(4)$ \\
\hline $\mathrm{N}(9)-\mathrm{H}(9)$ & 0.8600 \\
\hline$N(10)-C(36) \# 1$ & $1.31(5)$ \\
\hline $\mathrm{N}(10)-\mathrm{H}(10)$ & 0.8600 \\
\hline $\mathrm{P}(1)-\mathrm{Pt}(1)$ & $2.244(12)$ \\
\hline$P(2)-P t(1)$ & $2.277(14)$ \\
\hline$P(3)-P t(2)$ & $2.294(14)$ \\
\hline$P(4)-P t(2)$ & $2.266(12)$ \\
\hline$P(5)-P t(3)$ & $2.277(14)$ \\
\hline$P(6)-P t(4)$ & $2.257(13)$ \\
\hline $\operatorname{Pt}(3)-\mathrm{N}(6) \# 1$ & $1.99(3)$ \\
\hline $\operatorname{Pt}(3)-P(5) \# 1$ & $2.277(14)$ \\
\hline $\operatorname{Pt}(4)-\mathrm{N}(3)$ \# 1 & $2.11(3)$ \\
\hline $\operatorname{Pt}(4)-P(6) \# 1$ & $2.257(13)$ \\
\hline$O(1)-O(26)$ & $1.14(6)$ \\
\hline$O(1)-O(7)$ & $1.39(4)$ \\
\hline$O(1)-O(10)$ & $1.46(5)$ \\
\hline$O(2)-O(44)$ & $1.02(5)$ \\
\hline$O(2)-O(20)$ & $1.41(5)$ \\
\hline$O(3)-O(43)$ & $1.39(8)$ \\
\hline$O(4)-O(33)$ & $1.51(6)$ \\
\hline$O(5)-O(30)$ & $1.33(7)$ \\
\hline$O(5)-O(22)$ & $1.43(8)$ \\
\hline$O(5)-O(29)$ & $1.55(17)$ \\
\hline$O(8)-O(41)$ & $1.51(10)$ \\
\hline$O(12)-O(42)$ & $1.64(8)$ \\
\hline$O(13)-O(34)$ & $1.31(7)$ \\
\hline$O(14)-O(31)$ & $1.51(12)$ \\
\hline$O(17)-O(28)$ & $1.32(11)$ \\
\hline$O(19)-O(43)$ & $1.79(10)$ \\
\hline$O(24)-O(29)$ & $1.69(19)$ \\
\hline$O(37)-O(40)$ & $1.68(10)$ \\
\hline
\end{tabular}

Symmetry transformations used to generate equivalent atoms: $\# 1 \mathrm{x},-\mathrm{y}+3 / 2, \mathrm{z}$

Table S18. Bond angles [deg] for $6 \mathbf{a}$.

$\begin{array}{ll}\mathrm{C}(2)-\mathrm{C}(1)-\mathrm{N}(2) & 123(4) \\ \mathrm{C}(2)-\mathrm{C}(1)-\mathrm{H}(1) & 118.3 \\ \mathrm{~N}(2)-\mathrm{C}(1)-\mathrm{H}(1) & 118.3 \\ \mathrm{C}(1)-\mathrm{C}(2)-\mathrm{C}(3) & 124(4) \\ \mathrm{C}(1)-\mathrm{C}(2)-\mathrm{H}(2) & 117.9 \\ \mathrm{C}(3)-\mathrm{C}(2)-\mathrm{H}(2) & 117.9 \\ \mathrm{C}(4)-\mathrm{C}(3)-\mathrm{C}(2) & 118(4) \\ \mathrm{C}(4)-\mathrm{C}(3)-\mathrm{C}(6) & 118(4) \\ \mathrm{C}(2)-\mathrm{C}(3)-\mathrm{C}(6) & 124(4) \\ \mathrm{C}(3)-\mathrm{C}(4)-\mathrm{C}(5) & 116(4) \\ \mathrm{C}(3)-\mathrm{C}(4)-\mathrm{H}(4) & 121.8 \\ \mathrm{C}(5)-\mathrm{C}(4)-\mathrm{H}(4) & 121.8 \\ \mathrm{~N}(2)-\mathrm{C}(5)-\mathrm{C}(4) & 132(5) \\ \mathrm{N}(2)-\mathrm{C}(5)-\mathrm{H}(5) & 114.1 \\ \mathrm{C}(4)-\mathrm{C}(5)-\mathrm{H}(5) & 114.1 \\ \mathrm{C}(7)-\mathrm{C}(6)-\mathrm{C}(3) & 129(3) \\ \mathrm{C}(7)-\mathrm{C}(6)-\mathrm{N}(1) & 108(3)\end{array}$




\begin{tabular}{|c|c|}
\hline $\mathrm{C}(3)-\mathrm{C}(6)-\mathrm{N}(1)$ & $121(3)$ \\
\hline$C(6)-C(7)-C(8)$ & $107(3)$ \\
\hline$C(6)-C(7)-S(2)$ & $133(3)$ \\
\hline$C(8)-C(7)-S(2)$ & $120(3)$ \\
\hline$C(7)-C(8)-C(9)$ & $112(3)$ \\
\hline$C(7)-C(8)-S(1)$ & $7(3)$ \\
\hline$C(9)-C(8)-S(1)$ & $1(3)$ \\
\hline$N(1)-C(9)-C(10)$ & $8(3)$ \\
\hline$N(1)-C(9)-C(8)$ & $6(3)$ \\
\hline$C(10)-C(9)-C(8)$ & $27(3)$ \\
\hline$C(14)-C(10)-C(9)$ & $26(4)$ \\
\hline$C(14)-C(10)-C(11)$ & $2(3)$ \\
\hline$C(9)-C(10)-C(11)$ & $2(3)$ \\
\hline$C(12)-C(11)-C(10)$ & $8(4)$ \\
\hline $\mathrm{C}(12)-\mathrm{C}(11)-\mathrm{H}(11)$ & 121.0 \\
\hline $\mathrm{C}(10)-\mathrm{C}(11)-\mathrm{H}(11)$ & 1.0 \\
\hline $\mathrm{C}(11)-\mathrm{C}(12)-\mathrm{N}(3)$ & $28(4)$ \\
\hline $\mathrm{C}(11)-\mathrm{C}(12)-\mathrm{H}(12)$ & 6.0 \\
\hline $\mathrm{N}(3)-\mathrm{C}(12)-\mathrm{H}(12)$ & 6.0 \\
\hline$C(14)-C(13)-N(3)$ & $4(4)$ \\
\hline $\mathrm{C}(14)-\mathrm{C}(13)-\mathrm{H}(13)$ & 2.8 \\
\hline $\mathrm{N}(3)-\mathrm{C}(13)-\mathrm{H}(13)$ & 122.8 \\
\hline$C(13)-C(14)-C(10)$ & $130(4)$ \\
\hline $\mathrm{C}(13)-\mathrm{C}(14)-\mathrm{H}(14)$ & 114.8 \\
\hline $\mathrm{C}(10)-\mathrm{C}(14)-\mathrm{H}(14)$ & 114.8 \\
\hline$C(16)-C(15)-S(1)$ & $126(3)$ \\
\hline$C(16)-C(15)-S(2)$ & $117(3)$ \\
\hline$S(1)-C(15)-S(2)$ & $118(2)$ \\
\hline$C(15)-C(16)-S(3)$ & $120(4)$ \\
\hline$C(15)-C(16)-S(4)$ & $126(4)$ \\
\hline$S(3)-C(16)-S(4)$ & $114(3)$ \\
\hline$C(18)-C(17)-C(25)$ & $109(4)$ \\
\hline$C(18)-C(17)-S(3)$ & $116(3)$ \\
\hline$C(25)-C(17)-S(3)$ & $135(3)$ \\
\hline $\mathrm{C}(17)-\mathrm{C}(18)-\mathrm{C}(19)$ & $113(4)$ \\
\hline$C(17)-C(18)-S(4)$ & $121(3)$ \\
\hline$C(19)-C(18)-S(4)$ & $126(3)$ \\
\hline$C(20)-C(19)-N(4)$ & $126(4)$ \\
\hline$C(20)-C(19)-C(18)$ & $135(4)$ \\
\hline $\mathrm{N}(4)-\mathrm{C}(19)-\mathrm{C}(18)$ & $99(4)$ \\
\hline$C(19)-C(20)-C(24)$ & $119(4)$ \\
\hline$C(19)-C(20)-C(21)$ & $122(4)$ \\
\hline$C(24)-C(20)-C(21)$ & $117(4)$ \\
\hline$C(20)-C(21)-C(22)$ & $120(4)$ \\
\hline $\mathrm{C}(20)-\mathrm{C}(21)-\mathrm{H}(21)$ & 119.8 \\
\hline $\mathrm{C}(22)-\mathrm{C}(21)-\mathrm{H}(21)$ & 119.8 \\
\hline$N(5)-C(22)-C(21)$ & $121(4)$ \\
\hline$N(5)-C(22)-H(22)$ & 119.7 \\
\hline $\mathrm{C}(21)-\mathrm{C}(22)-\mathrm{H}(22)$ & 119.7 \\
\hline$C(24)-C(23)-N(5)$ & $119(5)$ \\
\hline $\mathrm{C}(24)-\mathrm{C}(23)-\mathrm{H}(23)$ & 120.6 \\
\hline $\mathrm{N}(5)-\mathrm{C}(23)-\mathrm{H}(23)$ & 120.6 \\
\hline$C(23)-C(24)-C(20)$ & $124(4)$ \\
\hline $\mathrm{C}(23)-\mathrm{C}(24)-\mathrm{H}(24)$ & 118.0 \\
\hline $\mathrm{C}(20)-\mathrm{C}(24)-\mathrm{H}(24)$ & 118.0 \\
\hline$C(26)-C(25)-N(4)$ & $127(4)$ \\
\hline$C(26)-C(25)-C(17)$ & $133(4)$ \\
\hline$N(4)-C(25)-C(17)$ & $100(3)$ \\
\hline$C(30)-C(26)-C(25)$ & $118(4)$ \\
\hline$C(30)-C(26)-C(27)$ & $118(4)$ \\
\hline$C(25)-C(26)-C(27)$ & $125(4)$ \\
\hline$C(26)-C(27)-C(28)$ & $111(4)$ \\
\hline
\end{tabular}




\begin{tabular}{|c|c|}
\hline $\mathrm{C}(26)-\mathrm{C}(27)-\mathrm{H}(27)$ & 124.6 \\
\hline $\mathrm{C}(28)-\mathrm{C}(27)-\mathrm{H}(27)$ & 124.6 \\
\hline$N(6)-C(28)-C(27)$ & $137(6)$ \\
\hline $\mathrm{N}(6)-\mathrm{C}(28)-\mathrm{H}(28)$ & 111.7 \\
\hline $\mathrm{C}(27)-\mathrm{C}(28)-\mathrm{H}(28)$ & 111.7 \\
\hline$C(30)-C(29)-N(6)$ & $120(4)$ \\
\hline $\mathrm{C}(30)-\mathrm{C}(29)-\mathrm{H}(29)$ & 120.2 \\
\hline$N(6)-C(29)-H(29)$ & 120.2 \\
\hline$C(26)-C(30)-C(29)$ & $126(4)$ \\
\hline$C(26)-C(30)-H(30)$ & 117.1 \\
\hline $\mathrm{C}(29)-\mathrm{C}(30)-\mathrm{H}(30)$ & 117.1 \\
\hline$N(7)-C(31)-C(32)$ & $121(4)$ \\
\hline $\mathrm{N}(7)-\mathrm{C}(31)-\mathrm{H}(31)$ & 119.5 \\
\hline $\mathrm{C}(32)-\mathrm{C}(31)-\mathrm{H}(31)$ & 119.5 \\
\hline$C(33)-C(32)-C(31)$ & $116(4)$ \\
\hline $\mathrm{C}(33)-\mathrm{C}(32)-\mathrm{H}(32)$ & 122.0 \\
\hline $\mathrm{C}(31)-\mathrm{C}(32)-\mathrm{H}(32)$ & 122.0 \\
\hline$C(32)-C(33)-C(34)$ & $118(4)$ \\
\hline$C(32)-C(33)-C(36)$ & $123(4)$ \\
\hline$C(34)-C(33)-C(36)$ & $118(4)$ \\
\hline$C(35)-C(34)-C(33)$ & $121(4)$ \\
\hline $\mathrm{C}(35)-\mathrm{C}(34)-\mathrm{H}(34)$ & 119.4 \\
\hline $\mathrm{C}(33)-\mathrm{C}(34)-\mathrm{H}(34)$ & 119.4 \\
\hline$C(34)-C(35)-N(7)$ & $120(4)$ \\
\hline $\mathrm{C}(34)-\mathrm{C}(35)-\mathrm{H}(35)$ & 120.2 \\
\hline $\mathrm{N}(7)-\mathrm{C}(35)-\mathrm{H}(35)$ & 120.2 \\
\hline$N(10)-C(36)-C(33)$ & $130(4)$ \\
\hline$N(10)-C(36)-C(37)$ & $107(4)$ \\
\hline$C(33)-C(36)-C(37)$ & $123(4)$ \\
\hline$C(37) \# 1-C(37)-C(36)$ & $104(2)$ \\
\hline C (37) \#1-C (37) -S (5) & $119.3(15)$ \\
\hline$C(36)-C(37)-S(5)$ & $135(3)$ \\
\hline$C(39)-C(38)-S(5)$ & $124.5(19)$ \\
\hline$C(39)-C(38)-S(5) \# 1$ & $124.5(19)$ \\
\hline$S(5)-C(38)-S(5) \# 1$ & $109(3)$ \\
\hline$C(38)-C(39)-S(6)$ & $119.6(16)$ \\
\hline$C(38)-C(39)-S(6) \# 1$ & $119.6(16)$ \\
\hline$S(6)-C(39)-S(6) \# 1$ & $121(3)$ \\
\hline$C(41)-C(40)-C(40) \# 1$ & $107(2)$ \\
\hline$C(41)-C(40)-S(6)$ & $137(3)$ \\
\hline$C(40) \# 1-C(40)-S(6)$ & $116.1(13)$ \\
\hline$N(9)-C(41)-C(40)$ & $107(4)$ \\
\hline$N(9)-C(41)-C(42)$ & $123(3)$ \\
\hline$C(40)-C(41)-C(42)$ & $128(3)$ \\
\hline$C(41)-C(42)-C(43)$ & $120(2)$ \\
\hline$C(41)-C(42)-C(46)$ & $117(2)$ \\
\hline$C(43)-C(42)-C(46)$ & $112(2)$ \\
\hline$C(44)-C(43)-C(42)$ & $110(3)$ \\
\hline $\mathrm{C}(44)-\mathrm{C}(43)-\mathrm{H}(43)$ & 125.0 \\
\hline $\mathrm{C}(42)-\mathrm{C}(43)-\mathrm{H}(43)$ & 125.0 \\
\hline$N(8)-C(44)-C(43)$ & $133(4)$ \\
\hline $\mathrm{N}(8)-\mathrm{C}(44)-\mathrm{H}(44)$ & 113.5 \\
\hline $\mathrm{C}(43)-\mathrm{C}(44)-\mathrm{H}(44)$ & 113.5 \\
\hline$N(8)-C(45)-C(46)$ & $129(4)$ \\
\hline $\mathrm{N}(8)-\mathrm{C}(45)-\mathrm{H}(45)$ & 115.3 \\
\hline$C(46)-C(45)-H(45)$ & 115.3 \\
\hline$C(45)-C(46)-C(42)$ & $117(4)$ \\
\hline $\mathrm{C}(45)-\mathrm{C}(46)-\mathrm{H}(46)$ & 121.3 \\
\hline $\mathrm{C}(42)-\mathrm{C}(46)-\mathrm{H}(46)$ & 121.3 \\
\hline$C(48)-C(47)-P(1)$ & $118(6)$ \\
\hline$C(50)-C(49)-P(1)$ & $125(5)$ \\
\hline$C(52)-C(51)-P(1)$ & $114(4)$ \\
\hline
\end{tabular}


$\mathrm{C}(54)-\mathrm{C}(53)-\mathrm{P}(2) \quad 113(5)$

$C(56)-C(55)-P(2) \quad 107(4)$

$\mathrm{C}(59)-\mathrm{C}(58)-\mathrm{P}(3) \quad 156(8)$

$\mathrm{P}(3)-\mathrm{C}(60)-\mathrm{C}(61) \quad 112(3)$

$\mathrm{C}(63)-\mathrm{C}(62)-\mathrm{P}(3) \quad 129(10)$

$C(65)-C(64)-P(4) \quad 105(4)$

$\mathrm{C}(67)-\mathrm{C}(66)-\mathrm{P}(4) \quad 110(3)$

$\mathrm{C}(69)-\mathrm{C}(68)-\mathrm{P}(4) \quad 128(6)$

$\mathrm{C}(74)-\mathrm{C}(73)-\mathrm{P}(6) \quad 122(4)$

$\mathrm{C}(9)-\mathrm{N}(1)-\mathrm{C}(6) \quad 107(3)$

$\mathrm{C}(9)-\mathrm{N}(1)-\mathrm{H}(1 \mathrm{~A}) \quad 126.6$

$\mathrm{C}(6)-\mathrm{N}(1)-\mathrm{H}(1 \mathrm{~A}) \quad 126.6$

$\mathrm{C}(5)-\mathrm{N}(2)-\mathrm{C}(1) \quad 106(4)$

$\mathrm{C}(5)-\mathrm{N}(2)-\mathrm{Pt}(1) \quad 132(3)$

$\mathrm{C}(1)-\mathrm{N}(2)-\mathrm{Pt}(1) \quad 122(2)$

$\mathrm{C}(12)-\mathrm{N}(3)-\mathrm{C}(13) \quad 116(4)$

$\mathrm{C}(12)-\mathrm{N}(3)-\mathrm{Pt}(4) \quad 123(3)$

$\mathrm{C}(13)-\mathrm{N}(3)-\mathrm{Pt}(4) \quad 121(3)$

$\mathrm{C}(19)-\mathrm{N}(4)-\mathrm{C}(25) \quad 119(4)$

$\mathrm{C}(19)-\mathrm{N}(4)-\mathrm{H}(4 \mathrm{~A}) \quad 120.4$

$\mathrm{C}(25)-\mathrm{N}(4)-\mathrm{H}(4 \mathrm{~A}) \quad 120.4$

$\mathrm{C}(22)-\mathrm{N}(5)-\mathrm{C}(23) \quad 118(4)$

$\mathrm{C}(22)-\mathrm{N}(5)-\mathrm{Pt}(2) \quad 118(3)$

$\mathrm{C}(23)-\mathrm{N}(5)-\mathrm{Pt}(2) \quad 121(3)$

$\mathrm{C}(28)-\mathrm{N}(6)-\mathrm{C}(29) \quad 109(4)$

$\mathrm{C}(28)-\mathrm{N}(6)-\mathrm{Pt}(3) \quad 127(4)$

$\mathrm{C}(29)-\mathrm{N}(6)-\mathrm{Pt}(3) \quad 123(3)$

$\mathrm{C}(31)-\mathrm{N}(7)-\mathrm{C}(35) \quad 124(3)$

$\mathrm{C}(31)-\mathrm{N}(7)-\mathrm{Pt}(1) \quad 119(3)$

$\mathrm{C}(35)-\mathrm{N}(7)-\mathrm{Pt}(1) \quad 116(2)$

$\mathrm{C}(44)-\mathrm{N}(8)-\mathrm{C}(45) \quad 117(3)$

$\mathrm{C}(44)-\mathrm{N}(8)-\mathrm{Pt}(2) \quad 125(3)$

$\mathrm{C}(45)-\mathrm{N}(8)-\mathrm{Pt}(2) \quad 117(2)$

$\mathrm{C}(41)-\mathrm{N}(9)-\mathrm{C}(41)$ \# $113(5)$

$\mathrm{C}(41)-\mathrm{N}(9)-\mathrm{H}(9) \quad 123.7$

$\mathrm{C}(41) \# 1-\mathrm{N}(9)-\mathrm{H}(9) \quad 123.7$

$\mathrm{C}(36)-\mathrm{N}(10)-\mathrm{C}(36) \# 1 \quad 116(5)$

$\mathrm{C}(36)-\mathrm{N}(10)-\mathrm{H}(10) \quad 122.1$

$\mathrm{C}(36) \# 1-\mathrm{N}(10)-\mathrm{H}(10) \quad 122.1$

$\mathrm{C}(49)-\mathrm{P}(1)-\mathrm{C}(47) \quad 104(3)$

$C(49)-P(1)-C(51) \quad 98(3)$

$\mathrm{C}(47)-\mathrm{P}(1)-\mathrm{C}(51) \quad 109(3)$

$\mathrm{C}(49)-\mathrm{P}(1)-\mathrm{Pt}(1) \quad 115(2)$

$\mathrm{C}(47)-\mathrm{P}(1)-\mathrm{Pt}(1) \quad 112(3)$

$\mathrm{C}(51)-\mathrm{P}(1)-\mathrm{Pt}(1) \quad 118(2)$

$C(57)-P(2)-C(55) \quad 105(2)$

$C(57)-P(2)-C(53) \quad 102(3)$

$\mathrm{C}(55)-\mathrm{P}(2)-\mathrm{C}(53) \quad 108(3)$

$\mathrm{C}(57)-\mathrm{P}(2)-\mathrm{Pt}(1) \quad 110.8(18)$

$\mathrm{C}(55)-\mathrm{P}(2)-\mathrm{Pt}(1) \quad 115.4(17)$

$\mathrm{C}(53)-\mathrm{P}(2)-\mathrm{Pt}(1) \quad 114(2)$

$C(58)-P(3)-C(60) \quad 101(3)$

$C(58)-P(3)-C(62) \quad 102(4)$

$C(60)-P(3)-C(62) \quad 100(4)$

$\mathrm{C}(58)-\mathrm{P}(3)-\mathrm{Pt}(2) \quad 109.5(18)$

$\mathrm{C}(60)-\mathrm{P}(3)-\mathrm{Pt}(2) \quad 111.1(19)$

$\mathrm{C}(62)-\mathrm{P}(3)-\mathrm{Pt}(2) \quad 129(4)$

$C(68)-P(4)-C(66) \quad 110(3)$

$\mathrm{C}(68)-\mathrm{P}(4)-\mathrm{C}(64) \quad 103(3)$

$C(66)-P(4)-C(64) \quad 104(2)$

$\mathrm{C}(68)-\mathrm{P}(4)-\mathrm{Pt}(2) \quad 109(2)$

$\mathrm{C}(66)-\mathrm{P}(4)-\mathrm{Pt}(2) \quad 111.6(17)$ 


\begin{tabular}{|c|c|}
\hline$C(64)-P(4)-P t(2)$ & $118.9(1$ \\
\hline$C(71)-P(5)-C(70)$ & $121(4)$ \\
\hline$C(71)-P(5)-C(72)$ & $92(4)$ \\
\hline$C(70)-P(5)-C(72)$ & $113(5)$ \\
\hline$C(71)-P(5)-P t(3)$ & $110(2)$ \\
\hline$C(70)-P(5)-P t(3)$ & $111(3)$ \\
\hline$C(72)-P(5)-P t(3)$ & $108(4)$ \\
\hline$C(73)-P(6)-C(76)$ & $94(4)$ \\
\hline$(73)-P(6)-C(75)$ & $87(3)$ \\
\hline$(76)-P(6)-C(75)$ & $125(4)$ \\
\hline$(73)-P(6)-P t(4)$ & $116.1(19$ \\
\hline$(76)-P(6)-P t(4)$ & $120(3)$ \\
\hline$(75)-P(6)-P t(4)$ & $108(2)$ \\
\hline $\mathrm{N}(2)-\operatorname{Pt}(1)-\mathrm{N}(7)$ & $82.7(12)$ \\
\hline$N(2)-P t(1)-P(1)$ & $90.4(9)$ \\
\hline$N(7)-P t(1)-P(1)$ & $173.1(9)$ \\
\hline$N(2)-P t(1)-P(2)$ & $172.3(9)$ \\
\hline $\mathrm{J}(7)-\mathrm{Pt}(1)-\mathrm{P}(2)$ & $90.2(9)$ \\
\hline$P(1)-P t(1)-P(2)$ & $96.7(5)$ \\
\hline$N(5)-P t(2)-N(8)$ & $84.4(12$ \\
\hline$N(5)-P t(2)-P(4)$ & $91.0(10)$ \\
\hline$N(8)-P t(2)-P(4)$ & $173.6(9)$ \\
\hline$N(5)-P t(2)-P(3)$ & $171.9(10$ \\
\hline$N(8)-P t(2)-P(3)$ & $87.7(9)$ \\
\hline$P(4)-P t(2)-P(3)$ & $96.7(5)$ \\
\hline$N(6)-P t(3)-N(6) \# 1$ & $82.1(19)$ \\
\hline$N(6)-P t(3)-P(5)$ & $90.4(10)$ \\
\hline$N(6) \# 1-P t(3)-P(5)$ & $172.5(10$ \\
\hline$N(6)-P t(3)-P(5) \# 1$ & $172.5(10$ \\
\hline$N(6) \# 1-P t(3)-P(5) \# 1$ & $90.4(10)$ \\
\hline$P(5)-P t(3)-P(5) \# 1$ & $97.1(8)$ \\
\hline$N(3)-\operatorname{Pt}(4)-N(3) \# 1$ & $78(2)$ \\
\hline$N(3)-P t(4)-P(6)$ & $92.6(12$ \\
\hline $\mathrm{N}(3) \# 1-\mathrm{Pt}(4)-\mathrm{P}(6)$ & $170.1(11$ \\
\hline$N(3)-P t(4)-P(6) \# 1$ & $170.1(11$ \\
\hline $\mathrm{N}(3) \# 1-\mathrm{Pt}(4)-\mathrm{P}(6) \# 1$ & $92.6(12$ \\
\hline$P(6)-P t(4)-P(6) \# 1$ & $97.1(8)$ \\
\hline$O(26)-O(1)-O(7)$ & $108(4)$ \\
\hline$O(26)-O(1)-O(10)$ & $114(4)$ \\
\hline$O(7)-O(1)-O(10)$ & $113(3)$ \\
\hline$O(44)-O(2)-O(20)$ & $110(4)$ \\
\hline$O(30)-O(5)-O(22)$ & $137(6)$ \\
\hline$O(30)-O(5)-O(29)$ & $135(10)$ \\
\hline$O(22)-O(5)-O(29)$ & $89(9)$ \\
\hline$O(5)-O(29)-O(24)$ & $88(10)$ \\
\hline$O(3)-O(43)-O(19)$ & $108(6)$ \\
\hline$C(15)-S(1)-C(8)$ & $93.1(18$ \\
\hline$C(7)-S(2)-C(15)$ & $91.5(18$ \\
\hline$C(17)-S(3)-C(16)$ & $98(2)$ \\
\hline$C(18)-S(4)-C(16)$ & $90(2)$ \\
\hline$C(37)-S(5)-C(38)$ & $94(2)$ \\
\hline$C(39)-S(6)-C(40)$ & $93(2)$ \\
\hline
\end{tabular}

Symmetry transformations used to generate equivalent atoms: \# $1 \mathrm{x},-\mathrm{y}+3 / 2, \mathrm{z}$

Table S9. Anisotropic displacement parameters for $\mathbf{6 a}$.

The anisotropic displacement factor exponent takes the form:

$-2 \mathrm{pi}^{\wedge} 2\left[\mathrm{~h}^{\wedge} 2 \mathrm{a}^{* \wedge} 2 \mathrm{U} 11+\ldots+2 \mathrm{~h} \mathrm{k} \mathrm{a}^{*} \mathrm{~b}^{*} \mathrm{U} 12\right]$ 


\begin{tabular}{|c|c|c|c|c|c|c|}
\hline $\begin{array}{c}N(1) \\
0.01(2)\end{array}$ & $0.08(2)$ & $0.10(2)$ & $0.08(2)$ & $0.017(19)$ & $0.014(18)$ & - \\
\hline $\mathrm{N}(2)$ & $0.06(2)$ & $0.049(19)$ & $0.22(4)$ & $-0.04(2)$ & $-0.08(3)$ & \\
\hline $0.035(18)$ & & & & & & \\
\hline $\begin{array}{c}N(3) \\
0.01(2)\end{array}$ & $0.09(3)$ & $0.12(3)$ & $0.11(3)$ & $0.05(2)$ & $-0.03(2)$ & \\
\hline $\begin{array}{c}N(4) \\
0.006(17)\end{array}$ & $0.07(2)$ & $0.064(19)$ & $0.07(2)$ & $0.009(16)$ & $-0.043(17)$ & - \\
\hline $\begin{array}{c}N(6) \\
0.02(2)\end{array}$ & $0.09(3)$ & $0.10(3)$ & $0.08(2)$ & $0.006(19)$ & $-0.02(2)$ & \\
\hline $\begin{array}{c}N(7) \\
0.000(16)\end{array}$ & $0.05(2)$ & $0.06(2)$ & $0.13(3)$ & $0.045(18)$ & $0.019(18)$ & \\
\hline $\begin{array}{c}N(8) \\
0.021(14)\end{array}$ & $0.06(2)$ & $0.018(13)$ & $0.11(2)$ & $0.009(14)$ & $0.021(17)$ & - \\
\hline$N(9)$ & $0.02(3)$ & $0.27(8)$ & $0.12(5)$ & 0.000 & $0.00(3)$ & 0.000 \\
\hline $\mathrm{N}(10)$ & $0.04(3)$ & $0.03(2)$ & $0.15(4)$ & 0.000 & $-0.02(2)$ & 0.000 \\
\hline $\begin{array}{c}P(1) \\
0.029(8)\end{array}$ & $0.153(14)$ & $0.056(7)$ & $0.129(11)$ & $0.001(7)$ & $0.025(10)$ & - \\
\hline $\begin{array}{c}P(2) \\
0.007(9)\end{array}$ & $0.138(13)$ & $0.098(10)$ & $0.097(10)$ & $0.015(7)$ & $0.017(8)$ & - \\
\hline $\begin{array}{c}P(3) \\
0.007(8)\end{array}$ & $0.143(13)$ & $0.064(8)$ & $0.156(13)$ & $0.031(8)$ & $-0.060(11)$ & - \\
\hline $\begin{array}{c}P(4) \\
0.013(7)\end{array}$ & $0.092(10)$ & $0.054(7)$ & $0.208(16)$ & $-0.007(8)$ & $-0.025(10)$ & \\
\hline $\begin{array}{c}P(5) \\
0.006(10)\end{array}$ & $0.173(16)$ & $0.093(10)$ & $0.118(12)$ & $-0.018(8)$ & $0.016(10)$ & - \\
\hline $\begin{array}{c}P(6) \\
0.010(10)\end{array}$ & $0.178(17)$ & $0.089(10)$ & $0.149(14)$ & $-0.043(9)$ & $-0.064(12)$ & \\
\hline $\begin{array}{c}\text { Pt (1) } \\
0.0111(9)\end{array}$ & $0.0980(14)$ & $0.0533(9)$ & $0.0752(12)$ & $0.0075(8)$ & $0.0080(9)$ & - \\
\hline $\begin{array}{c}\text { Pt (2) } \\
0.0025(9)\end{array}$ & $0.0769(12)$ & $0.0481(9)$ & $0.1289(16)$ & $0.0033(9)$ & $-0.0150(10)$ & \\
\hline Pt (3) & $0.099(2)$ & $0.0818(17)$ & $0.095(2)$ & 0.000 & $0.0208(15)$ & 0.000 \\
\hline Pt ( 4) & $0.102(2)$ & $0.0773(16)$ & $0.0779(17)$ & 0.000 & $-0.0257(14)$ & 0.000 \\
\hline $\begin{array}{c}S(1) \\
0.011(6)\end{array}$ & $0.062(7)$ & $0.110(9)$ & $0.094(8)$ & $0.024(7)$ & $0.020(6)$ & \\
\hline $\begin{array}{c}S(2) \\
0.010(7)\end{array}$ & $0.058(7)$ & $0.145(11)$ & $0.092(8)$ & $0.025(7)$ & $0.003(6)$ & \\
\hline $\begin{array}{c}S(3) \\
0.010(8)\end{array}$ & $0.060(8)$ & $0.172(13)$ & $0.143(12)$ & $0.063(10)$ & $0.025(7)$ & \\
\hline $\begin{array}{c}S(4) \\
0.015(8)\end{array}$ & $0.055(7)$ & $0.164(13)$ & $0.123(10)$ & $0.049(9)$ & $0.015(7)$ & \\
\hline $\begin{array}{c}S(5) \\
0.004(5)\end{array}$ & $0.053(7)$ & $0.044(6)$ & $0.264(17)$ & $0.000(8)$ & $-0.004(9)$ & \\
\hline $\begin{array}{c}S(6) \\
0.007(6)\end{array}$ & $0.065(8)$ & $0.067(7)$ & $0.231(16)$ & $-0.017(8)$ & $0.021(9)$ & - \\
\hline
\end{tabular}

Table S10. Crystal data and structure refinement for $\mathbf{7 b}$

Empirical formula

Formula weight

Temperature

Wavelength

Crystal system, space group

Unit cell dimensions

\section{C44 H39 Cl2 F6 N3 O6 P2 Pt S4}

1275.95

200(2) K

$0.71073 \mathrm{~A}$

Triclinic, $\mathrm{P}-1$

$\mathrm{a}=13.222(2)$ A alpha $=82.95(1) \mathrm{deg}$.

$\mathrm{b}=13.956(2) \mathrm{A} \quad$ beta $=85.70(1) \mathrm{deg}$.

$\mathrm{c}=14.574(2) \mathrm{A} \quad$ gamma $=70.87(1) \mathrm{deg}$. 
Volume

Z, Calculated density

Absorption coefficient

$\mathrm{F}(000)$

Crystal size

Theta range for data collection

Limiting indices

Reflections collected / unique

Completeness to theta $=27.55$

Absorption correction

Max. and min. transmission

Refinement method

Data / restraints / parameters

Goodness-of-fit on $\mathrm{F}^{\wedge} 2$

Final $R$ indices [I $>2 \operatorname{sigma}(\mathrm{I})]$

$\mathrm{R}$ indices (all data)

Largest diff. peak and hole
2519.8(6) $\mathrm{A}^{\wedge} 3$

2, $1.682 \mathrm{Mg} / \mathrm{m}^{\wedge} 3$

$3.190 \mathrm{~mm}^{\wedge}-1$

1264

$0.08 \times 0.02 \times 0.02 \mathrm{~mm}$

2.82 to $27.55 \mathrm{deg}$.

$-16<=\mathrm{h}<=17,-18<=\mathrm{k}<=17,-18<=\mathrm{l}<=18$

$37355 / 11428$ [R(int $)=0.1064]$

$98.3 \%$

Semi-empirical from equivalents

0.938 and 0.651

Full-matrix least-squares on $\mathrm{F}^{\wedge} 2$

11428 / 1 / 625

1.110

$\mathrm{R} 1=0.0679, \mathrm{wR} 2=0.1251[7718 \mathrm{Fo}]$

$\mathrm{R} 1=0.1251, \mathrm{wR} 2=0.1511$

2.745 and -1.096 e. $\mathrm{A}^{\wedge}-3$
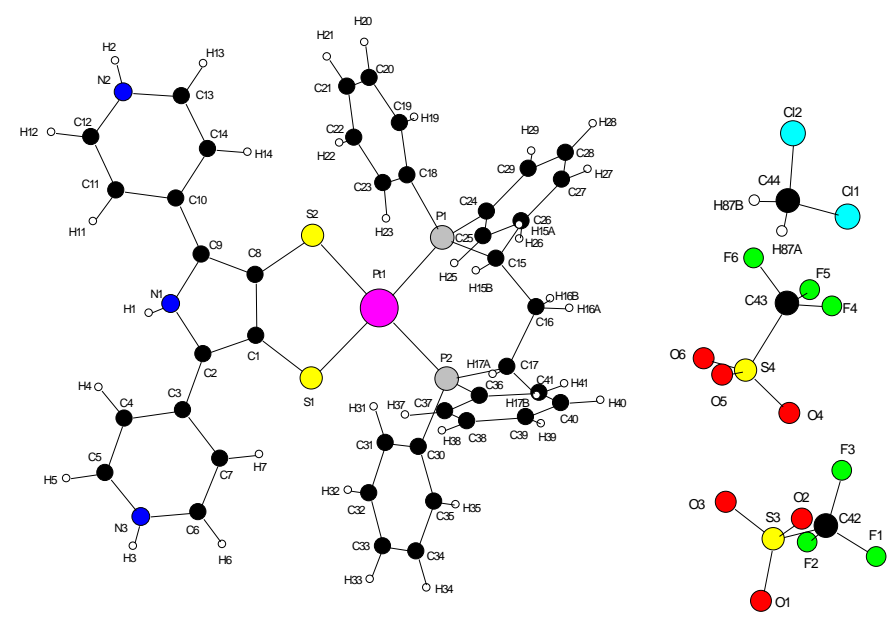

Figure S3422. X-Ray structure of dithiolene 7b

Table S11. Atomic coordinates and equivalent isotropic displacement parameters for $\mathbf{7 b}$.

$\mathrm{U}(\mathrm{eq})$ is defined as one third of the trace of the orthogonalized Uij tensor.

\begin{tabular}{lrlll}
\hline & & & \\
& $\mathrm{x}$ & $\mathrm{y}$ & $\mathrm{U}($ eq) \\
\hline $\mathrm{C}(1)$ & $0.0450(7)$ & $0.4806(7)$ & $0.8457(6)$ & $0.0244(19)$ \\
$\mathrm{C}(2)$ & $0.0177(7)$ & $0.4116(7)$ & $0.9162(5)$ & $0.0220(18)$ \\
$\mathrm{C}(3)$ & $0.0804(7)$ & $0.3161(7)$ & $0.9616(5)$ & $0.0222(18)$ \\
$\mathrm{C}(4)$ & $0.0400(8)$ & $0.2723(7)$ & $1.0420(6)$ & $0.030(2)$ \\
$\mathrm{C}(5)$ & $0.1024(8)$ & $0.1817(8)$ & $1.0860(7)$ & $0.039(2)$ \\
$\mathrm{C}(6)$ & $0.2427(8)$ & $0.1718(8)$ & $0.9753(7)$ & $0.040(2)$ \\
$\mathrm{C}(7)$ & $0.1839(8)$ & $0.2619(8)$ & $0.9279(6)$ & $0.034(2)$ \\
$\mathrm{C}(8)$ & $-0.0491(7)$ & $0.5613(7)$ & $0.8219(5)$ & $0.0236(18)$
\end{tabular}




\begin{tabular}{|c|c|c|c|c|}
\hline$C(9)$ & $-0.1355(7)$ & $0.5459(7)$ & $0.8793(6)$ & $0.0240(18)$ \\
\hline C (10) & $-0.2426(7)$ & $0.6103(7)$ & $0.8918(6)$ & $0.0253(19)$ \\
\hline C (11) & $-0.3062(7)$ & $0.5948(7)$ & $0.9706(6)$ & $0.030(2)$ \\
\hline $\mathrm{C}(12)$ & $-0.4042(8)$ & $0.6656(8)$ & $0.9878(7)$ & $0.040(2)$ \\
\hline$C(13)$ & $-0.3858(8)$ & $0.7679(8)$ & $0.8509(6)$ & $0.035(2)$ \\
\hline C (14) & $-0.2869(7)$ & $0.6993(7)$ & $0.8323(6)$ & $0.031(2)$ \\
\hline C (15) & $0.1877(9)$ & $0.8045(8)$ & $0.5353(7)$ & $0.040(3)$ \\
\hline C (16) & $0.2837(9)$ & $0.7311(9)$ & $0.4888(7)$ & $0.046(3)$ \\
\hline C (17) & $0.3552(8)$ & $0.6490(8)$ & $0.5571(7)$ & $0.038(2)$ \\
\hline C (18) & $-0.0244(8)$ & $0.8594(7)$ & $0.6160(6)$ & $0.033(2)$ \\
\hline C (19) & $-0.1287(10)$ & $0.8889(9)$ & $0.5895(9)$ & $0.054(3)$ \\
\hline$C(20)$ & $-0.2064(12)$ & $0.9718(11)$ & $0.6281(12)$ & $0.079(5)$ \\
\hline $\mathrm{C}(21)$ & $-0.1778(16)$ & $1.0216(10)$ & $0.6925(10)$ & $0.078(6)$ \\
\hline $\mathrm{C}(22)$ & $-0.0764(12)$ & $0.9924(9)$ & $0.7189(9)$ & $0.057(3)$ \\
\hline C (23) & $0.0012(11)$ & $0.9097(8)$ & $0.6825(8)$ & $0.051(3)$ \\
\hline C (24) & $0.0398(9)$ & $0.7229(8)$ & $0.4696(6)$ & $0.036(2)$ \\
\hline$C(25)$ & $0.0441(10)$ & $0.6253(9)$ & $0.4573(7)$ & $0.047(3)$ \\
\hline$C(26)$ & $0.0241(14)$ & $0.6003(11)$ & $0.3728(8)$ & $0.071(4)$ \\
\hline C (27) & $-0.0037(18)$ & $0.6767(13)$ & $0.3021(9)$ & $0.104(7)$ \\
\hline $\mathrm{C}(28)$ & $-0.0128(16)$ & $0.7738(12)$ & $0.3114(8)$ & $0.086(6)$ \\
\hline C (29) & $0.0102(13)$ & $0.7990(10)$ & $0.3954(8)$ & $0.066(4)$ \\
\hline C (30) & $0.4011(7)$ & $0.5012(7)$ & $0.7096(6)$ & $0.030(2)$ \\
\hline C ( 31$)$ & $0.3982(9)$ & $0.5571(8)$ & $0.7811(7)$ & $0.040(2)$ \\
\hline C (32) & $0.4820(10)$ & $0.5258(10)$ & $0.8422(8)$ & $0.054(3)$ \\
\hline C (33) & $0.5641(9)$ & $0.4383(9)$ & $0.8350(7)$ & $0.045(3)$ \\
\hline C (34) & $0.5685(8)$ & $0.3799(9)$ & $0.7633(8)$ & $0.045(3)$ \\
\hline$C(35)$ & $0.4862(9)$ & $0.4102(8)$ & $0.7000(7)$ & $0.040(2)$ \\
\hline C (36) & $0.3090(8)$ & $0.4592(8)$ & $0.5472(6)$ & $0.035(2)$ \\
\hline C (37) & $0.2798(10)$ & $0.3752(9)$ & $0.5802(8)$ & $0.053(3)$ \\
\hline C (38) & $0.2806(13)$ & $0.3023(11)$ & $0.5247(9)$ & $0.067(4)$ \\
\hline C (39) & $0.3135(12)$ & $0.3108(11)$ & $0.4328(9)$ & $0.066(4)$ \\
\hline$C(40)$ & $0.3446(11)$ & $0.3917(11)$ & $0.3992(8)$ & $0.057(3)$ \\
\hline C ( 41$)$ & $0.3440(9)$ & $0.4659(10)$ & $0.4544(7)$ & $0.046(3)$ \\
\hline C ( 42$)$ & $-0.1779(13)$ & $1.1564(13)$ & $0.9588(13)$ & $0.082(5)$ \\
\hline$C(43)$ & $-0.5068(10)$ & $1.1086(9)$ & $0.7381(9)$ & $0.050(3)$ \\
\hline C ( 44$)$ & $0.3226(14)$ & $0.9157(13)$ & $0.7334(11)$ & $0.086(5)$ \\
\hline $\mathrm{N}(1)$ & $-0.0911(6)$ & $0.4551(6)$ & $0.9361(5)$ & $0.0236(16)$ \\
\hline $\mathrm{N}(2)$ & $-0.4402(8)$ & $0.7496(8)$ & $0.9298(7)$ & $0.045(2)$ \\
\hline $\mathrm{N}(3)$ & $0.1989(8)$ & $0.1350(7)$ & $1.0531(6)$ & $0.037(2)$ \\
\hline$O(1)$ & $-0.1691(8)$ & $1.1956(8)$ & $1.1260(7)$ & $0.073(3)$ \\
\hline$O(2)$ & $-0.2271(6)$ & $1.3342(6)$ & $1.0034(6)$ & $0.049(2)$ \\
\hline$O(3)$ & $-0.3420(6)$ & $1.2363(7)$ & $1.0571(7)$ & $0.067(3)$ \\
\hline$O(4)$ & $-0.3598(6)$ & $1.0095(7)$ & $0.8487(6)$ & $0.064(3)$ \\
\hline$O(5)$ & $-0.5029(8)$ & $1.1531(7)$ & $0.9018(6)$ & $0.065(2)$ \\
\hline$O(6)$ & $-0.5394(7)$ & $0.9983(7)$ & $0.8818(6)$ & $0.062(2)$ \\
\hline$F(1)$ & $-0.0733(7)$ & $1.1516(10)$ & $0.9363(8)$ & $0.128(5)$ \\
\hline$F(2)$ & $-0.1768(11)$ & 1.0631 ( 8 ) & $0.9859(12)$ & $0.172(7)$ \\
\hline$F(3)$ & $-0.2287(9)$ & $1.1839(10)$ & $0.8802(8)$ & $0.130(5)$ \\
\hline$F(4)$ & $-0.4480(7)$ & 1.1662 (6) & $0.6982(5)$ & $0.072(2)$ \\
\hline$F(5)$ & $-0.4904(8)$ & $1.0331(7)$ & $0.6857(5)$ & $0.088(3)$ \\
\hline$F(6)$ & $-0.6100(7)$ & 1.1648 ( 8) & $0.7319(6)$ & $0.101(3)$ \\
\hline $\mathrm{P}(1)$ & $0.0820(2)$ & $0.75033(19)$ & $0.57547(16)$ & $0.0263(5)$ \\
\hline $\mathrm{P}(2)$ & $0.29860(19)$ & 0.55637 (19) & $0.62314(16)$ & $0.0272(5)$ \\
\hline Cl (1) & $0.4244(6)$ & $0.8392(5)$ & $0.6726(6)$ & $0.164(3)$ \\
\hline Cl (2) & $0.2393(6)$ & $1.0163(5)$ & $0.6637(6)$ & $0.159(3)$ \\
\hline Pt (1) & $0.12942(3)$ & $0.61412(3)$ & $0.68390(3)$ & $0.02410(10)$ \\
\hline S (1) & $0.17224(18)$ & $0.47794(19)$ & 0.79967 (15) & $0.0287(5)$ \\
\hline$S(2)$ & $-0.04893(18)$ & $0.66104(18)$ & $0.73698(15)$ & $0.0274(5)$ \\
\hline$S(3)$ & $-0.2341(2)$ & $1.2393(2)$ & $1.0462(2)$ & $0.0419(6)$ \\
\hline
\end{tabular}




\begin{tabular}{|c|c|c|c|c|}
\hline$S(4)$ & $-0.4734(2)$ & $1.0620(2)$ & $0.85665(18)$ & $0.0390(6)$ \\
\hline $\mathrm{H}(4)$ & -0.0286 & 0.3046 & 1.0654 & 0.036 \\
\hline $\mathrm{H}(5)$ & 0.0760 & 0.1531 & 1.1395 & 0.046 \\
\hline $\mathrm{H}(6)$ & 0.3115 & 0.1367 & 0.9543 & 0.048 \\
\hline $\mathrm{H}(7)$ & 0.2122 & 0.2872 & 0.8735 & 0.041 \\
\hline $\mathrm{H}(11)$ & -0.2816 & 0.5362 & 1.0114 & 0.036 \\
\hline $\mathrm{H}(12)$ & -0.4454 & 0.6546 & 1.0402 & 0.048 \\
\hline $\mathrm{H}(13)$ & -0.4147 & 0.8256 & 0.8101 & 0.042 \\
\hline $\mathrm{H}(14)$ & -0.2483 & 0.7121 & 0.7789 & 0.037 \\
\hline $\mathrm{H}(15 \mathrm{~A})$ & 0.1580 & 0.8642 & 0.4919 & 0.048 \\
\hline $\mathrm{H}(15 \mathrm{~B})$ & 0.2118 & 0.8267 & 0.5877 & 0.048 \\
\hline $\mathrm{H}(16 \mathrm{~A})$ & 0.3261 & 0.7695 & 0.4546 & 0.055 \\
\hline $\mathrm{H}(16 \mathrm{~B})$ & 0.2584 & 0.6982 & 0.4447 & 0.055 \\
\hline $\mathrm{H}(17 \mathrm{~A})$ & 0.3788 & 0.6832 & 0.6011 & 0.046 \\
\hline $\mathrm{H}(17 \mathrm{~B})$ & 0.4184 & 0.6111 & 0.5228 & 0.046 \\
\hline $\mathrm{H}(19)$ & -0.1481 & 0.8547 & 0.5465 & 0.065 \\
\hline $\mathrm{H}(20)$ & -0.2769 & 0.9926 & 0.6100 & 0.094 \\
\hline $\mathrm{H}(21)$ & -0.2292 & 1.0759 & 0.7178 & 0.094 \\
\hline $\mathrm{H}(22)$ & -0.0574 & 1.0273 & 0.7615 & 0.069 \\
\hline $\mathrm{H}(23)$ & 0.0707 & 0.8883 & 0.7031 & 0.061 \\
\hline $\mathrm{H}(25)$ & 0.0608 & 0.5748 & 0.5069 & 0.056 \\
\hline $\mathrm{H}(26)$ & 0.0294 & 0.5337 & 0.3646 & 0.085 \\
\hline $\mathrm{H}(27)$ & -0.0170 & 0.6609 & 0.2449 & 0.124 \\
\hline $\mathrm{H}(28)$ & -0.0342 & 0.8240 & 0.2621 & 0.104 \\
\hline $\mathrm{H}(29)$ & 0.0059 & 0.8657 & 0.4020 & 0.079 \\
\hline $\mathrm{H}(31)$ & 0.3406 & 0.6158 & 0.7890 & 0.048 \\
\hline $\mathrm{H}(32)$ & 0.4813 & 0.5659 & 0.8889 & 0.064 \\
\hline H (33) & 0.6176 & 0.4173 & 0.8780 & 0.054 \\
\hline $\mathrm{H}(34)$ & 0.6260 & 0.3207 & 0.7572 & 0.054 \\
\hline $\mathrm{H}(35)$ & 0.4879 & 0.3708 & 0.6525 & 0.048 \\
\hline $\mathrm{H}(37)$ & 0.2587 & 0.3674 & 0.6423 & 0.063 \\
\hline $\mathrm{H}(38)$ & 0.2589 & 0.2471 & 0.5490 & 0.080 \\
\hline H (39) & 0.3143 & 0.2618 & 0.3947 & 0.079 \\
\hline $\mathrm{H}(40)$ & 0.3670 & 0.3978 & 0.3374 & 0.069 \\
\hline $\mathrm{H}(41)$ & 0.3669 & 0.5203 & 0.4297 & 0.056 \\
\hline $\mathrm{H}(44 \mathrm{~A})$ & 0.3518 & 0.9429 & 0.7794 & 0.103 \\
\hline $\mathrm{H}(44 \mathrm{~B})$ & 0.2807 & 0.8757 & 0.7656 & 0.103 \\
\hline $\mathrm{H}(1)$ & $-0.106(7)$ & $0.412(7)$ & $0.961(6)$ & $0.02(2)$ \\
\hline $\mathrm{H}(2)$ & $-0.498(3)$ & $0.783(8)$ & $0.926(8)$ & $0.05(4)$ \\
\hline $\mathrm{H}(3)$ & $0.237(8)$ & $0.093(8)$ & $1.076(7)$ & $0.03(3)$ \\
\hline
\end{tabular}

Table S12. Bond lengths [A] for $\mathbf{7 b}$.

\begin{tabular}{ll}
\hline $\mathrm{C}(1)-\mathrm{C}(8)$ & $1.409(12)$ \\
$\mathrm{C}(1)-\mathrm{C}(2)$ & $1.430(12)$ \\
$\mathrm{C}(1)-\mathrm{S}(1)$ & $1.754(9)$ \\
$\mathrm{C}(2)-\mathrm{N}(1)$ & $1.390(11)$ \\
$\mathrm{C}(2)-\mathrm{C}(3)$ & $1.429(12)$ \\
$\mathrm{C}(3)-\mathrm{C}(4)$ & $1.406(12)$ \\
$\mathrm{C}(3)-\mathrm{C}(7)$ & $1.413(12)$ \\
$\mathrm{C}(4)-\mathrm{C}(5)$ & $1.374(13)$ \\
$\mathrm{C}(4)-\mathrm{H}(4)$ & 0.9300 \\
$\mathrm{C}(5)-\mathrm{N}(3)$ & $1.312(14)$ \\
$\mathrm{C}(5)-\mathrm{H}(5)$ & 0.9300 \\
$\mathrm{C}(6)-\mathrm{N}(3)$ & $1.357(13)$
\end{tabular}


$C(6)-C(7)$

$\mathrm{C}(6)-\mathrm{H}(6)$

$\mathrm{C}(7)-\mathrm{H}(7)$

$\mathrm{C}(8)-\mathrm{C}(9)$

$C(8)-S(2)$

$\mathrm{C}(9)-\mathrm{N}(1)$

C (9) $-\mathrm{C}(10)$

C (10)-C (14)

C (10)-C (11)

$C(11)-C(12)$

$\mathrm{C}(11)-\mathrm{H}(11)$

$\mathrm{C}(12)-\mathrm{N}(2)$

$\mathrm{C}(12)-\mathrm{H}(12)$

$\mathrm{C}(13)-\mathrm{N}(2)$

C (13)-C (14)

$\mathrm{C}(13)-\mathrm{H}(13)$

$\mathrm{C}(14)-\mathrm{H}(14)$

$\mathrm{C}(15)-\mathrm{C}(16)$

$C(15)-P(1)$

$\mathrm{C}(15)-\mathrm{H}(15 \mathrm{~A})$

$\mathrm{C}(15)-\mathrm{H}(15 \mathrm{~B})$

C (16)-C (17)

$\mathrm{C}(16)-\mathrm{H}(16 \mathrm{~A})$

$\mathrm{C}(16)-\mathrm{H}(16 \mathrm{~B})$

$\mathrm{C}(17)-\mathrm{P}(2)$

$\mathrm{C}(17)-\mathrm{H}(17 \mathrm{~A})$

$\mathrm{C}(17)-\mathrm{H}(17 \mathrm{~B})$

C (18)-C (19)

C (18)-C (23)

$\mathrm{C}(18)-\mathrm{P}(1)$

C (19) - C (20)

$\mathrm{C}(19)-\mathrm{H}(19)$

C (20)-C (21)

C (20) $-\mathrm{H}(20)$

C (21) - C (22)

$\mathrm{C}(21)-\mathrm{H}(21)$

C (22) $-\mathrm{C}(23)$

$\mathrm{C}(22)-\mathrm{H}(22)$

$\mathrm{C}(23)-\mathrm{H}(23)$

C (24)-C (25)

C (24)-C (29)

$C(24)-P(1)$

C (25)-C (26)

$\mathrm{C}(25)-\mathrm{H}(25)$

C (26) $-\mathrm{C}(27)$

$\mathrm{C}(26)-\mathrm{H}(26)$

C (27)-C (28)

C (27) $-\mathrm{H}(27)$

C (28)-C (29)

$\mathrm{C}(28)-\mathrm{H}(28)$

C (29) $-\mathrm{H}(29)$

C (30)-C (31)

C (30)-C (35)

$C(30)-P(2)$

C (31) - C (32)

$\mathrm{C}(31)-\mathrm{H}(31)$

C (32) $-\mathrm{C}(33)$

C (32) $-\mathrm{H}(32)$

C (33)-C (34)
1.374(13)

0.9300

0.9300

1. $422(12)$

$1.746(9)$

1.401 (11)

$1.419(12)$

$1.404(12)$

$1.409(12)$

1.375 (13)

0.9300

1.330 (14)

0.9300

1. 352 (13)

1. 375 (13)

0.9300

0.9300

1.520 (15)

$1.824(10)$

0.9700

0.9700

1.533 (14)

0.9700

0.9700

1.837(10)

0.9700

0.9700

1.374(16)

1.385 (15)

$1.827(10)$

1. 416 (19)

0.9300

1.37(2)

0.9300

1.34(2)

0.9300

1.399(16)

0.9300

0.9300

$1.379(15)$

1.401 (15)

$1.803(10)$

1.386 (15)

0.9300

1. 366 (19)

0.9300

1.34(2)

0.9300

1.394(16)

0.9300

0.9300

1.369(14)

1.408 (14)

1.831 (9)

1.395 (15)

0.9300

1.351 (16)

0.9300

1.389(16) 


\begin{tabular}{|c|c|}
\hline $\mathrm{C}(33)-\mathrm{H}(33)$ & 0.9300 \\
\hline$C(34)-C(35)$ & $1.402(15)$ \\
\hline $\mathrm{C}(34)-\mathrm{H}(34)$ & 0.9300 \\
\hline $\mathrm{C}(35)-\mathrm{H}(35)$ & 0.9300 \\
\hline$C(36)-C(37)$ & $1.374(15)$ \\
\hline$C(36)-C(41)$ & $1.398(13)$ \\
\hline$C(36)-P(2)$ & $1.818(11)$ \\
\hline$C(37)-C(38)$ & $1.373(16)$ \\
\hline $\mathrm{C}(37)-\mathrm{H}(37)$ & 0.9300 \\
\hline$C(38)-C(39)$ & $1.380(17)$ \\
\hline $\mathrm{C}(38)-\mathrm{H}(38)$ & 0.9300 \\
\hline$C(39)-C(40)$ & $1.349(18)$ \\
\hline $\mathrm{C}(39)-\mathrm{H}(39)$ & 0.9300 \\
\hline$C(40)-C(41)$ & $1.385(17)$ \\
\hline $\mathrm{C}(40)-\mathrm{H}(40)$ & 0.9300 \\
\hline $\mathrm{C}(41)-\mathrm{H}(41)$ & 0.9300 \\
\hline$C(42)-F(2)$ & $1.31(2)$ \\
\hline$C(42)-F(3)$ & $1.322(18)$ \\
\hline$C(42)-E(1)$ & $1.378(18)$ \\
\hline$C(42)-S(3)$ & $1.781(15)$ \\
\hline$C(43)-E(5)$ & $1.327(14)$ \\
\hline$C(43)-F(6)$ & $1.336(14)$ \\
\hline$C(43)-F(4)$ & $1.348(14)$ \\
\hline$C(43)-S(4)$ & $1.804(12)$ \\
\hline$C(44)-C 1(1)$ & $1.687(19)$ \\
\hline$C(44)-C l(2)$ & $1.737(16)$ \\
\hline $\mathrm{C}(44)-\mathrm{H}(44 \mathrm{~A})$ & 0.9700 \\
\hline $\mathrm{C}(44)-\mathrm{H}(44 \mathrm{~B})$ & 0.9700 \\
\hline $\mathrm{N}(1)-\mathrm{H}(1)$ & $0.74(9)$ \\
\hline $\mathrm{N}(2)-\mathrm{H}(2)$ & $0.75(2)$ \\
\hline $\mathrm{N}(3)-\mathrm{H}(3)$ & $0.70(10)$ \\
\hline$O(1)-S(3)$ & $1.448(9)$ \\
\hline$O(2)-S(3)$ & $1.421(8)$ \\
\hline$O(3)-S(3)$ & $1.437(8)$ \\
\hline$O(4)-S(4)$ & $1.442(8)$ \\
\hline$O(5)-S(4)$ & $1.430(8)$ \\
\hline$O(6)-S(4)$ & $1.438(9)$ \\
\hline $\mathrm{P}(1)-\mathrm{Pt}(1)$ & $2.266(2)$ \\
\hline $\mathrm{P}(2)-\mathrm{Pt}(1)$ & $2.266(2)$ \\
\hline $\operatorname{Pt}(1)-S(2)$ & $2.330(2)$ \\
\hline Pt (1) - S (1) & $2.330(2)$ \\
\hline
\end{tabular}

Table S13. Bond angles [deg] for $\mathbf{7 b}$.

\begin{tabular}{ll}
\hline$C(8)-C(1)-C(2)$ & $108.4(8)$ \\
$C(8)-C(1)-S(1)$ & $122.8(7)$ \\
$C(2)-C(1)-S(1)$ & $128.6(7)$ \\
$N(1)-C(2)-C(3)$ & $122.6(8)$ \\
$N(1)-C(2)-C(1)$ & $105.7(7)$ \\
$C(3)-C(2)-C(1)$ & $131.7(8)$ \\
$C(4)-C(3)-C(7)$ & $117.5(8)$ \\
$C(4)-C(3)-C(2)$ & $120.4(8)$ \\
$C(7)-C(3)-C(2)$ & $122.0(8)$ \\
$C(5)-C(4)-C(3)$ & $119.7(9)$ \\
$C(5)-C(4)-H(4)$ & 120.2 \\
$C(3)-C(4)-H(4)$ & 120.2 \\
$N(3)-C(5)-C(4)$ & $120.7(10)$
\end{tabular}


$\mathrm{N}(3)-\mathrm{C}(5)-\mathrm{H}(5)$

$\mathrm{C}(4)-\mathrm{C}(5)-\mathrm{H}(5)$

$\mathrm{N}(3)-\mathrm{C}(6)-\mathrm{C}(7)$

$\mathrm{N}(3)-\mathrm{C}(6)-\mathrm{H}(6)$

$\mathrm{C}(7)-\mathrm{C}(6)-\mathrm{H}(6)$

$C(6)-C(7)-C(3)$

$\mathrm{C}(6)-\mathrm{C}(7)-\mathrm{H}(7)$

$\mathrm{C}(3)-\mathrm{C}(7)-\mathrm{H}(7)$

$\mathrm{C}(1)-\mathrm{C}(8)-\mathrm{C}(9)$

$C(1)-C(8)-S(2)$

$C(9)-C(8)-S(2)$

$\mathrm{N}(1)-\mathrm{C}(9)-\mathrm{C}(10)$

$\mathrm{N}(1)-\mathrm{C}(9)-\mathrm{C}(8)$

$C(10)-C(9)-C(8)$

$C(14)-C(10)-C(11)$

$C(14)-C(10)-C(9)$

$C(11)-C(10)-C(9)$

$C(12)-C(11)-C(10)$

$\mathrm{C}(12)-\mathrm{C}(11)-\mathrm{H}(11)$

$\mathrm{C}(10)-\mathrm{C}(11)-\mathrm{H}(11)$

$\mathrm{N}(2)-\mathrm{C}(12)-\mathrm{C}(11)$

$\mathrm{N}(2)-\mathrm{C}(12)-\mathrm{H}(12)$

$\mathrm{C}(11)-\mathrm{C}(12)-\mathrm{H}(12)$

$\mathrm{N}(2)-\mathrm{C}(13)-\mathrm{C}(14)$

$\mathrm{N}(2)-\mathrm{C}(13)-\mathrm{H}(13)$

$\mathrm{C}(14)-\mathrm{C}(13)-\mathrm{H}(13)$

C (13) - C (14)-C (10)

$\mathrm{C}(13)-\mathrm{C}(14)-\mathrm{H}(14)$

$\mathrm{C}(10)-\mathrm{C}(14)-\mathrm{H}(14)$

$C(16)-C(15)-P(1)$

$\mathrm{C}(16)-\mathrm{C}(15)-\mathrm{H}(15 \mathrm{~A})$

$\mathrm{P}(1)-\mathrm{C}(15)-\mathrm{H}(15 \mathrm{~A})$

$\mathrm{C}(16)-\mathrm{C}(15)-\mathrm{H}(15 \mathrm{~B})$

$\mathrm{P}(1)-\mathrm{C}(15)-\mathrm{H}(15 \mathrm{~B})$

$\mathrm{H}(15 \mathrm{~A})-\mathrm{C}(15)-\mathrm{H}(15 \mathrm{~B})$

$C(15)-C(16)-C(17)$

$\mathrm{C}(15)-\mathrm{C}(16)-\mathrm{H}(16 \mathrm{~A})$

$\mathrm{C}(17)-\mathrm{C}(16)-\mathrm{H}(16 \mathrm{~A})$

$\mathrm{C}(15)-\mathrm{C}(16)-\mathrm{H}(16 \mathrm{~B})$

$\mathrm{C}(17)-\mathrm{C}(16)-\mathrm{H}(16 \mathrm{~B})$

$\mathrm{H}(16 \mathrm{~A})-\mathrm{C}(16)-\mathrm{H}(16 \mathrm{~B})$

$\mathrm{C}(16)-\mathrm{C}(17)-\mathrm{P}(2)$

$\mathrm{C}(16)-\mathrm{C}(17)-\mathrm{H}(17 \mathrm{~A})$

$\mathrm{P}(2)-\mathrm{C}(17)-\mathrm{H}(17 \mathrm{~A})$

$\mathrm{C}(16)-\mathrm{C}(17)-\mathrm{H}(17 \mathrm{~B})$

$\mathrm{P}(2)-\mathrm{C}(17)-\mathrm{H}(17 \mathrm{~B})$

$\mathrm{H}(17 \mathrm{~A})-\mathrm{C}(17)-\mathrm{H}(17 \mathrm{~B})$

$\mathrm{C}(19)-\mathrm{C}(18)-\mathrm{C}(23)$

$\mathrm{C}(19)-\mathrm{C}(18)-\mathrm{P}(1)$

$C(23)-C(18)-P(1)$

$C(18)-C(19)-C(20)$

$\mathrm{C}(18)-\mathrm{C}(19)-\mathrm{H}(19)$

$\mathrm{C}(20)-\mathrm{C}(19)-\mathrm{H}(19)$

$C(21)-C(20)-C(19)$

$\mathrm{C}(21)-\mathrm{C}(20)-\mathrm{H}(20)$

$\mathrm{C}(19)-\mathrm{C}(20)-\mathrm{H}(20)$

$C(22)-C(21)-C(20)$

$\mathrm{C}(22)-\mathrm{C}(21)-\mathrm{H}(21)$

C (20)-C (21) - H (21)
119.7

119.7

$119.0(10)$

120.5

120.5

$120.0(9)$

120.0

120.0

$108.4(8)$

$122.2(7)$

$129.4(7)$

$122.7(8)$

$105.8(8)$

$130.9(8)$

$116.1(8)$

$122.0(8)$

$121.6(8)$

$120.7(9)$

119.6

119.6

120.1 (9)

119.9

119.9

118.7 (9)

120.7

120.7

121.7 (8)

119.1

119.1

114.0 (8)

108.8

108.8

108.8

108.8

107.7

113.3 ( 8 )

108.9

108.9

108.9

108.9

107.7

$118.2(7)$

107.8

107.8

107.8

107.8

107.1

$118.8(11)$

$123.9(9)$

117.1 (9)

119.6 (14)

120.2

120.2

120.2 (15)

119.9

119.9

$120.3(14)$

119.8

119.8 


\begin{tabular}{|c|c|}
\hline$C(21)-C(22)-C(23)$ & $120.3(14)$ \\
\hline $\mathrm{C}(21)-\mathrm{C}(22)-\mathrm{H}(22)$ & 119.9 \\
\hline $\mathrm{C}(23)-\mathrm{C}(22)-\mathrm{H}(22)$ & 119.9 \\
\hline$C(18)-C(23)-C(22)$ & $120.8(13)$ \\
\hline $\mathrm{C}(18)-\mathrm{C}(23)-\mathrm{H}(23)$ & 119.6 \\
\hline $\mathrm{C}(22)-\mathrm{C}(23)-\mathrm{H}(23)$ & 119.6 \\
\hline$C(25)-C(24)-C(29)$ & $118.5(10)$ \\
\hline$C(25)-C(24)-P(1)$ & $120.9(8)$ \\
\hline $\mathrm{C}(29)-\mathrm{C}(24)-\mathrm{P}(1)$ & $120.4(8)$ \\
\hline$C(24)-C(25)-C(26)$ & $121.8(11)$ \\
\hline $\mathrm{C}(24)-\mathrm{C}(25)-\mathrm{H}(25)$ & 119.1 \\
\hline $\mathrm{C}(26)-\mathrm{C}(25)-\mathrm{H}(25)$ & 19.1 \\
\hline$C(27)-C(26)-C(25)$ & $117.7(12)$ \\
\hline $\mathrm{C}(27)-\mathrm{C}(26)-\mathrm{H}(26)$ & 121.1 \\
\hline $\mathrm{C}(25)-\mathrm{C}(26)-\mathrm{H}(26)$ & 121.1 \\
\hline$C(28)-C(27)-C(26)$ & $122.8(12)$ \\
\hline $\mathrm{C}(28)-\mathrm{C}(27)-\mathrm{H}(27)$ & 118.6 \\
\hline $\mathrm{C}(26)-\mathrm{C}(27)-\mathrm{H}(27)$ & 18.6 \\
\hline$C(27)-C(28)-C(29)$ & $119.8(13)$ \\
\hline $\mathrm{C}(27)-\mathrm{C}(28)-\mathrm{H}(28)$ & 120.1 \\
\hline $\mathrm{C}(29)-\mathrm{C}(28)-\mathrm{H}(28)$ & 120.1 \\
\hline$C(28)-C(29)-C(24)$ & $119.3(12)$ \\
\hline $\mathrm{C}(28)-\mathrm{C}(29)-\mathrm{H}(29)$ & 120.3 \\
\hline $\mathrm{C}(24)-\mathrm{C}(29)-\mathrm{H}(29)$ & 120.3 \\
\hline$C(31)-C(30)-C(35)$ & $120.0(9)$ \\
\hline$C(31)-C(30)-P(2)$ & $117.1(7)$ \\
\hline$C(35)-C(30)-P(2)$ & $122.6(8)$ \\
\hline$C(30)-C(31)-C(32)$ & $119.7(10)$ \\
\hline $\mathrm{C}(30)-\mathrm{C}(31)-\mathrm{H}(31)$ & 120.1 \\
\hline $\mathrm{C}(32)-\mathrm{C}(31)-\mathrm{H}(31)$ & 120.1 \\
\hline$C(33)-C(32)-C(31)$ & $121.3(11)$ \\
\hline $\mathrm{C}(33)-\mathrm{C}(32)-\mathrm{H}(32)$ & 119.3 \\
\hline $\mathrm{C}(31)-\mathrm{C}(32)-\mathrm{H}(32)$ & 119.3 \\
\hline$C(32)-C(33)-C(34)$ & $119.9(10)$ \\
\hline $\mathrm{C}(32)-\mathrm{C}(33)-\mathrm{H}(33)$ & 120.0 \\
\hline $\mathrm{C}(34)-\mathrm{C}(33)-\mathrm{H}(33)$ & 120.0 \\
\hline$C(33)-C(34)-C(35)$ & $120.0(10)$ \\
\hline $\mathrm{C}(33)-\mathrm{C}(34)-\mathrm{H}(34)$ & 120.0 \\
\hline $\mathrm{C}(35)-\mathrm{C}(34)-\mathrm{H}(34)$ & 120.0 \\
\hline$C(34)-C(35)-C(30)$ & $118.9(10)$ \\
\hline $\mathrm{C}(34)-\mathrm{C}(35)-\mathrm{H}(35)$ & 120.6 \\
\hline $\mathrm{C}(30)-\mathrm{C}(35)-\mathrm{H}(35)$ & 120.6 \\
\hline$C(37)-C(36)-C(41)$ & $117.0(10)$ \\
\hline$C(37)-C(36)-P(2)$ & $119.8(8)$ \\
\hline$C(41)-C(36)-P(2)$ & $123.2(9)$ \\
\hline$C(38)-C(37)-C(36)$ & $122.2(11)$ \\
\hline $\mathrm{C}(38)-\mathrm{C}(37)-\mathrm{H}(37)$ & 118.9 \\
\hline $\mathrm{C}(36)-\mathrm{C}(37)-\mathrm{H}(37)$ & 118.9 \\
\hline$C(37)-C(38)-C(39)$ & $120.1(12)$ \\
\hline $\mathrm{C}(37)-\mathrm{C}(38)-\mathrm{H}(38)$ & 119.9 \\
\hline $\mathrm{C}(39)-\mathrm{C}(38)-\mathrm{H}(38)$ & 119.9 \\
\hline$C(40)-C(39)-C(38)$ & $118.7(11)$ \\
\hline $\mathrm{C}(40)-\mathrm{C}(39)-\mathrm{H}(39)$ & 120.6 \\
\hline $\mathrm{C}(38)-\mathrm{C}(39)-\mathrm{H}(39)$ & 120.6 \\
\hline$C(39)-C(40)-C(41)$ & $121.8(11)$ \\
\hline $\mathrm{C}(39)-\mathrm{C}(40)-\mathrm{H}(40)$ & 119.1 \\
\hline $\mathrm{C}(41)-\mathrm{C}(40)-\mathrm{H}(40)$ & 119.1 \\
\hline$C(40)-C(41)-C(36)$ & $120.1(12)$ \\
\hline $\mathrm{C}(40)-\mathrm{C}(41)-\mathrm{H}(41)$ & 120.0 \\
\hline
\end{tabular}




\begin{tabular}{|c|c|}
\hline $\mathrm{C}(36)-\mathrm{C}(41)-\mathrm{H}(41)$ & 120.0 \\
\hline$F(2)-C(42)-F(3)$ & $106.6(13)$ \\
\hline$F(2)-C(42)-F(1)$ & $106.9(14)$ \\
\hline$F(3)-C(42)-F(1)$ & $105.8(16)$ \\
\hline$F(2)-C(42)-S(3)$ & $111.6(15)$ \\
\hline$F(3)-C(42)-S(3)$ & $114.2(11)$ \\
\hline$F(1)-C(42)-S(3)$ & $111.2(10)$ \\
\hline$F(5)-C(43)-F(6)$ & $106.8(11)$ \\
\hline$F(5)-C(43)-F(4)$ & $106.2(10)$ \\
\hline$F(6)-C(43)-F(4)$ & $107.8(10)$ \\
\hline$F(5)-C(43)-S(4)$ & $112.0(8)$ \\
\hline$F(6)-C(43)-S(4)$ & $111.2(9)$ \\
\hline$F(4)-C(43)-S(4)$ & $112.6(9)$ \\
\hline $\mathrm{Cl}(1)-\mathrm{C}(44)-\mathrm{Cl}(2)$ & $112.6(10)$ \\
\hline $\mathrm{Cl}(1)-\mathrm{C}(44)-\mathrm{H}(44 \mathrm{~A})$ & 109.1 \\
\hline $\mathrm{Cl}(2)-\mathrm{C}(44)-\mathrm{H}(44 \mathrm{~A})$ & 109.1 \\
\hline $\mathrm{Cl}(1)-\mathrm{C}(44)-\mathrm{H}(44 \mathrm{~B})$ & 109.1 \\
\hline $\mathrm{Cl}(2)-\mathrm{C}(44)-\mathrm{H}(44 \mathrm{~B})$ & 109.1 \\
\hline $\mathrm{H}(44 \mathrm{~A})-\mathrm{C}(44)-\mathrm{H}(44 \mathrm{~B})$ & 107.8 \\
\hline $\mathrm{C}(2)-\mathrm{N}(1)-\mathrm{C}(9)$ & $111.7(7)$ \\
\hline $\mathrm{C}(2)-\mathrm{N}(1)-\mathrm{H}(1)$ & $103(7)$ \\
\hline $\mathrm{C}(9)-\mathrm{N}(1)-\mathrm{H}(1)$ & $141(7)$ \\
\hline$C(12)-N(2)-C(13)$ & $122.6(9)$ \\
\hline $\mathrm{C}(12)-\mathrm{N}(2)-\mathrm{H}(2)$ & $126(10)$ \\
\hline $\mathrm{C}(13)-\mathrm{N}(2)-\mathrm{H}(2)$ & $109(10)$ \\
\hline$C(5)-N(3)-C(6)$ & $123.0(9)$ \\
\hline $\mathrm{C}(5)-\mathrm{N}(3)-\mathrm{H}(3)$ & $126(9)$ \\
\hline $\mathrm{C}(6)-\mathrm{N}(3)-\mathrm{H}(3)$ & $111(9)$ \\
\hline$C(24)-P(1)-C(15)$ & $102.9(5)$ \\
\hline$C(24)-P(1)-C(18)$ & $107.4(5)$ \\
\hline$C(15)-P(1)-C(18)$ & $102.9(5)$ \\
\hline$C(24)-P(1)-P t(1)$ & $113.7(3)$ \\
\hline$C(15)-P(1)-P t(1)$ & $115.5(4)$ \\
\hline $\mathrm{C}(18)-\mathrm{P}(1)-\mathrm{Pt}(1)$ & $113.3(3)$ \\
\hline$C(36)-P(2)-C(30)$ & $107.6(5)$ \\
\hline$C(36)-P(2)-C(17)$ & $106.4(5)$ \\
\hline$C(30)-P(2)-C(17)$ & $99.3(4)$ \\
\hline$C(36)-P(2)-P t(1)$ & $110.1(3)$ \\
\hline$C(30)-P(2)-P t(1)$ & $114.1(3)$ \\
\hline$C(17)-P(2)-P t(1)$ & $118.4(4)$ \\
\hline $\mathrm{P}(1)-\mathrm{Pt}(1)-\mathrm{P}(2)$ & $92.79(9)$ \\
\hline$P(1)-P t(1)-S(2)$ & $87.89(8)$ \\
\hline$P(2)-P t(1)-S(2)$ & $173.68(10)$ \\
\hline $\mathrm{P}(1)-\mathrm{Pt}(1)-\mathrm{S}(1)$ & $177.50(10)$ \\
\hline $\mathrm{P}(2)-\mathrm{Pt}(1)-\mathrm{S}(1)$ & $89.50(9)$ \\
\hline$S(2)-P t(1)-S(1)$ & $89.94(8)$ \\
\hline $\mathrm{C}(1)-\mathrm{S}(1)-\mathrm{Pt}(1)$ & $101.7(3)$ \\
\hline$C(8)-S(2)-P t(1)$ & $102.4(3)$ \\
\hline$O(2)-S(3)-O(3)$ & $113.4(5)$ \\
\hline$O(2)-S(3)-O(1)$ & $115.8(5)$ \\
\hline$O(3)-S(3)-O(1)$ & $114.8(6)$ \\
\hline$O(2)-S(3)-C(42)$ & $102.4(8)$ \\
\hline$O(3)-S(3)-C(42)$ & $102.5(7)$ \\
\hline$O(1)-S(3)-C(42)$ & $105.7(7)$ \\
\hline$O(5)-S(4)-O(6)$ & $115.0(6)$ \\
\hline$O(5)-S(4)-O(4)$ & $115.0(6)$ \\
\hline$O(6)-S(4)-O(4)$ & $115.8(6)$ \\
\hline$O(5)-S(4)-C(43)$ & $103.3(6)$ \\
\hline$O(6)-S(4)-C(43)$ & $102.6(6)$ \\
\hline
\end{tabular}


Table S14. Anisotropic displacement parameters for $\mathbf{7 b}$.

The anisotropic displacement factor exponent takes the form:

$-2 \mathrm{pi}^{\wedge} 2\left[\mathrm{~h}^{\wedge} 2 \mathrm{a}^{* \wedge} 2 \mathrm{U} 11+\ldots+2 \mathrm{~h} \mathrm{k} \mathrm{a}^{*} \mathrm{~b}^{*} \mathrm{U} 12\right]$

\begin{tabular}{|c|c|c|c|c|c|c|}
\hline & U11 & $\mathrm{U} 22$ & U33 & $\mathrm{U} 23$ & U13 & $\mathrm{U} 12$ \\
\hline $\begin{array}{c}\overline{C(1)} \\
0.002(4)\end{array}$ & $0.020(4)$ & $0.030(5)$ & $0.020(4)$ & $-0.003(4)$ & $-0.003(3)$ & - \\
\hline $\begin{array}{c}C(2) \\
0.005(4)\end{array}$ & $0.021(4)$ & $0.026(5)$ & $0.017(4)$ & $-0.003(3)$ & $0.001(3)$ & - \\
\hline $\begin{array}{c}C(3) \\
0.007(4)\end{array}$ & $0.019(4)$ & $0.028(5)$ & $0.019(4)$ & $0.000(3)$ & $-0.007(3)$ & - \\
\hline $\begin{array}{c}C(4) \\
0.016(4)\end{array}$ & $0.035(5)$ & $0.028(5)$ & $0.028(5)$ & $0.006(4)$ & $-0.002(4)$ & - \\
\hline $\begin{array}{c}C(5) \\
0.008(5)\end{array}$ & $0.037(6)$ & $0.041(6)$ & $0.033(5)$ & $0.011(5)$ & $-0.006(4)$ & - \\
\hline $\begin{array}{c}C(6) \\
0.001(4)\end{array}$ & $0.032(6)$ & $0.027(5)$ & $0.053(6)$ & $0.003(5)$ & $0.000(5)$ & - \\
\hline $\begin{array}{c}C(7) \\
0.010(5)\end{array}$ & $0.035(5)$ & $0.038(6)$ & $0.028(5)$ & $0.000(4)$ & $0.006(4)$ & - \\
\hline $\begin{array}{c}C(8) \\
0.009(4)\end{array}$ & $0.026(5)$ & $0.029(5)$ & $0.016(4)$ & $-0.003(3)$ & $0.000(3)$ & - \\
\hline $\begin{array}{c}C(9) \\
0.008(4)\end{array}$ & $0.030(5)$ & $0.021(4)$ & $0.021(4)$ & $-0.003(3)$ & $-0.002(3)$ & - \\
\hline $\begin{array}{c}C(10) \\
0.007(4)\end{array}$ & $0.023(4)$ & $0.026(5)$ & $0.026(4)$ & $0.000(4)$ & $-0.002(3)$ & - \\
\hline $\begin{array}{c}C(11) \\
0.008(4)\end{array}$ & $0.023(5)$ & $0.031(5)$ & $0.034(5)$ & $-0.001(4)$ & $0.003(4)$ & - \\
\hline $\begin{array}{c}C(12) \\
0.009(5)\end{array}$ & $0.035(6)$ & $0.041(6)$ & $0.042(6)$ & $-0.010(5)$ & $0.014(4)$ & - \\
\hline $\begin{array}{c}C(13) \\
0.000(4)\end{array}$ & $0.028(5)$ & $0.038(6)$ & $0.029(5)$ & $0.002(4)$ & $-0.004(4)$ & \\
\hline $\begin{array}{c}C(14) \\
0.008(4)\end{array}$ & $0.024(5)$ & $0.032(5)$ & $0.034(5)$ & $0.003(4)$ & $0.008(4)$ & - \\
\hline $\begin{array}{c}C(15) \\
0.019(5)\end{array}$ & $0.039(6)$ & $0.041(6)$ & $0.040(6)$ & $0.014(5)$ & $0.005(4)$ & - \\
\hline $\begin{array}{c}C(16) \\
0.020(5)\end{array}$ & $0.042(6)$ & $0.051(7)$ & $0.040(6)$ & $0.012(5)$ & $0.015(5)$ & - \\
\hline $\begin{array}{c}C(17) \\
0.022(5)\end{array}$ & $0.030(5)$ & $0.050(7)$ & $0.039(5)$ & $0.007(5)$ & $0.006(4)$ & - \\
\hline $\begin{array}{c}C(18) \\
0.009(4)\end{array}$ & $0.044(6)$ & $0.023(5)$ & $0.028(5)$ & $0.001(4)$ & $0.007(4)$ & - \\
\hline $\begin{array}{c}C(19) \\
0.009(6)\end{array}$ & $0.050(7)$ & $0.046(7)$ & $0.057(7)$ & $0.008(6)$ & $0.006(6)$ & - \\
\hline $\begin{array}{c}C(20) \\
0.003(7)\end{array}$ & $0.045(8)$ & $0.056(9)$ & $0.109(13)$ & $0.028(9)$ & $0.011(8)$ & \\
\hline $\begin{array}{c}C(21) \\
0.013(9)\end{array}$ & $0.129(16)$ & $0.037(8)$ & $0.048(8)$ & $0.007(6)$ & $0.043(9)$ & - \\
\hline $\begin{array}{c}C(22) \\
0.009(7)\end{array}$ & $0.074(10)$ & $0.034(7)$ & $0.055(7)$ & $-0.003(5)$ & $0.021(7)$ & - \\
\hline $\begin{array}{c}C(23) \\
0.012(6)\end{array}$ & $0.070(9)$ & $0.034(6)$ & $0.044(6)$ & $-0.010(5)$ & $0.016(6)$ & - \\
\hline $\begin{array}{c}C(24) \\
0.021(5)\end{array}$ & $0.048(6)$ & $0.039(6)$ & $0.023(5)$ & $0.007(4)$ & $0.000(4)$ & - \\
\hline$C(25)$ & $0.054(7)$ & $0.052(7)$ & $0.039(6)$ & $0.007(5)$ & $-0.008(5)$ & - \\
\hline
\end{tabular}


0.025 (6) C (26)

0.054 (9) C (27)

0.104 (15) C (28)

0.059 (11) C (29)

$0.046(8)$ C (30)

$0.011(4)$ C (31)

0.008 (5) C (32)

0.021 (7) C (33)

0.017 (6) C (34)

0.001 (5) C (35)

$0.010(5)$ C (36)

$0.006(5)$ C (37)

$0.035(7)$ C (38)

0.050 (9) C (39)

$0.033(8)$ C (40)

$0.016(7)$ C (41)

0.017 (6) C (42)

$0.010(8)$ C (43)

0.012 (6) C (44)

$0.066(12)$ $\mathrm{N}(1)$

0.009 (3) $\mathrm{N}(2)$

0.004 (4) $\mathrm{N}(3)$

0.007 (4) $\mathrm{O}(1)$

$0.034(5)$ O (2)

$0.023(4)$ O (3)

0.030 (4) O (4)

$0.003(4)$ $\mathrm{O}(5)$

$0.014(5)$ O (6)

0.019 (4) $\mathrm{F}(1)$ 0.001 (6)

\begin{tabular}{|c|c|c|c|c|}
\hline $0.129(14)$ & $0.061(9)$ & $0.042(7)$ & $-0.005(6)$ & $-0.023(8)$ \\
\hline $0.22(2)$ & $0.096(13)$ & $0.031(7)$ & $0.002(7)$ & $-0.030(10)$ \\
\hline $0.174(18)$ & $0.073(10)$ & $0.028(6)$ & $0.002(6)$ & $-0.029(8)$ \\
\hline $0.120(13)$ & $0.048(8)$ & $0.045(7)$ & $-0.002(6)$ & $-0.013(7)$ \\
\hline $0.022(5)$ & $0.031(5)$ & $0.036(5)$ & $0.007(4)$ & $0.000(4)$ \\
\hline $0.036(6)$ & $0.034(6)$ & $0.048(6)$ & $-0.008(5)$ & $-0.005(5)$ \\
\hline $0.061(8)$ & $0.053(8)$ & $0.048(7)$ & $0.004(6)$ & $-0.015(6)$ \\
\hline $0.036(6)$ & $0.056(7)$ & $0.044(6)$ & $0.000(5)$ & $-0.013(5)$ \\
\hline $0.027(5)$ & $0.040(6)$ & $0.057(7)$ & $0.001(5)$ & $0.000(5)$ \\
\hline $0.043(6)$ & $0.044(6)$ & $0.030(5)$ & $-0.001(4)$ & $-0.002(4)$ \\
\hline $0.025(5)$ & $0.047(6)$ & $0.029(5)$ & $-0.004(4)$ & $0.001(4)$ \\
\hline $0.070(9)$ & $0.057(8)$ & $0.042(6)$ & $-0.020(6)$ & $0.014(6)$ \\
\hline $0.108(12)$ & $0.062(9)$ & $0.048(7)$ & $-0.015(6)$ & $0.012(7)$ \\
\hline $0.097(11)$ & $0.059(9)$ & $0.055(8)$ & $-0.037(7)$ & $-0.001(7)$ \\
\hline $0.067(9)$ & $0.072(9)$ & $0.029(6)$ & $-0.011(6)$ & $0.003(5)$ \\
\hline $0.040(6)$ & $0.061(8)$ & $0.038(6)$ & $-0.003(5)$ & $0.007(5)$ \\
\hline $0.065(10)$ & $0.073(11)$ & $0.113(13)$ & $-0.056(10)$ & $-0.019(9)$ \\
\hline $0.045(7)$ & $0.042(7)$ & $0.058(7)$ & $0.005(6)$ & $-0.004(6)$ \\
\hline $0.103(13)$ & $0.104(13)$ & $0.071(10)$ & $0.020(9)$ & $-0.028(9)$ \\
\hline $0.022(4)$ & $0.023(4)$ & $0.025(4)$ & $0.005(3)$ & $0.000(3)$ \\
\hline $0.028(5)$ & $0.040(6)$ & $0.056(6)$ & $-0.005(5)$ & $0.005(4)$ \\
\hline $0.038(5)$ & $0.027(5)$ & $0.043(5)$ & $0.005(4)$ & $-0.012(4)$ \\
\hline $0.064(6)$ & $0.080(7)$ & $0.077(6)$ & $0.030(5)$ & $-0.028(5)$ \\
\hline $0.044(5)$ & $0.042(5)$ & $0.067(5)$ & $0.001(4)$ & $-0.001(4)$ \\
\hline $0.034(5)$ & $0.072(6)$ & $0.101(7)$ & $0.001(5)$ & $-0.007(4)$ \\
\hline $0.033(4)$ & $0.081(7)$ & $0.056(5)$ & $0.014(5)$ & $-0.002(4)$ \\
\hline $0.071(6)$ & $0.051(5)$ & $0.072(6)$ & $-0.028(5)$ & $0.011(5)$ \\
\hline $0.054(6)$ & $0.053(5)$ & $0.073(6)$ & $0.013(4)$ & $0.005(4)$ \\
\hline $2(6)$ & $0.167(11)$ & $0.162(10)$ & $-0.116(9)$ & $0.016(6)$ \\
\hline
\end{tabular}




$\begin{array}{lllllll}F(2) & 0.147(12) & 0.053(6) & 0.33(2) & -0.079(10) & -0.038(12) & - \\ 0.023(7) & & & & & & \\ F(3) & 0.112(9) & 0.173(12) & 0.117(9) & -0.083(8) & -0.020(7) & - \\ 0.033(8) & 0.088(6) & 0.055(5) & 0.069(5) & 0.014(4) & 0.000(4) & - \\ F(4) & 0.08 & \end{array}$

$0.028(4)$ $\mathrm{F}(5)$

$0.049(6)$ $\mathrm{F}(6)$

$0.007(6)$ $\mathrm{P}(1)$

$0.088(6)$

$0.055(5)$

$0.069(5)$

$0.130(8) \quad 0.100(7)$

$0.045(4)$

$0.068(6)$

$0.127(8)$

$0.081(6)$

$-0.021(4)$

$-0.004(5)$

$.0093(11)$

$\mathrm{P}(2)$

$0.0289(13) \quad 0.0263(13) \quad 0.0225(11) \quad 0.0013(9) \quad 0.0022(9) \quad-$

$0.0232(12)$

$0.0318(14)$

$0.0259(12)$

2) $0.0004(10)$

(10) $0.0029(9)-$

$0.0099(11)$ $\mathrm{Cl}(1)$

$0.054(5)$

$\begin{array}{lllllll}0.156(6) & 0.137(5) & 0.226(8) & -0.072(5) & -0.063(6) & -\end{array}$

$\mathrm{Cl}(2)$

$0.072(4)$

Pt (1)

$0.00919(13)$ $\mathrm{S}(1)$

$0.144(6)$

$0.122(5)$

$0.228(7)$

$0.057(5) \quad-0.097(5) \quad-$

0.02148 (17) 0.02856 (19) 0.02155 (16) 0.00165 (12) 0.00182 (11$0.0216(12) \quad 0.0332(13) 0.0268(11) \quad 0.0073(10) \quad 0.0014(9) \quad-$ $0.0068(10)$ $\mathrm{S}(2)$

$0.0032(10)$ $\mathrm{S}(3)$

$0.0238(12) \quad 0.0259(12) \quad 0.0255(11) \quad 0.0071(9) \quad 0.0039(9) \quad$ -

$0.0290(13) 0.0398(15) 0.0590(17)-0.0019(13) 0.0000(12)-$ $0.0154(12)$ $\mathrm{S}(4)$

$0.0346(14) \quad 0.0344(14) 0.0398(14) 0.0010(11) \quad 0.0013(11)-$ $0.0024(11)$ 
1. J. Y. Balandier, A. Belyasmine and M. Salle, Synthesis-Stuttgart, 2006, 2815-2817.

2. J. O. Jeppesen, K. Takimiya, F. Jensen and J. Becher, Organic letters, 1999, 1, 1291-1294.

3. P. J. Stang, D. H. Cao, S. Saito and A. M. Arif, J Am Chem Soc, 1995, 117, 6273-6283.

4. T. G. Appleton, M. A. Bennett and I. B. Tomkins, Journal of the Chemical Society, Dalton Transactions, 1976, $439-446$. 